\title{
GUÍA ILUSTRADA PARA LA IDENTIFICACIÓN DE LAS FAMILIAS Y LOS GÉNEROS DE LOS ANFÍPODOS DEL SUBORDEN GAMMARIDEA DE LA PENÍNSULA IBÉRICA
}

\author{
M. Ortiz * y A. Jimeno **
}

\begin{abstract}
RESUMEN
Se trata de una guía ilustrada para la identificación de las Familias y los Géneros de los anfípodos del Suborden Gammaridea de la Península Ibérica. Abarca 40 Familias y 142 Géneros. Se inicia con una clave maestra de 10 láminas ilustradas que permite identificar las diferentes Familias. Siguen 66 láminas con numerosos dibujos que permiten confirmar las Familias e identificar los Géneros. El uso de imágenes evita los problemas de terminología y de subjetividad de las claves dicotómicas tradicionales.
\end{abstract}

Palabras clave: Sistemática, Crustacea, Amphipoda, Gammaridea, Guía ilustrada, Península Iberica.

\section{ABSTRACT \\ Pictorial guide for the identification of the families and genera of Gammaridea Amphipods of the Iberian Peninsula}

An original pictorial guide for the identification of the Families and Genera of the amphipod crustaceans of the Sub Order Gammaridea, from the Iberian Peninsula is presented. The guide includes a total of 40 Families and 142 Genera. When using the guide, it is necessary only, to compare the specimen to be classified with a Master key of 10 plates, for the Family identification. The Master Key is followed by an alphabetically ordered 66 plates, which allow to confirm the Families, and to select the Genera. This system, avoid problems of terminology, and the subjectivity of the classical dichotomic keys.

Key words: Systematic, Crustacea, Amphipoda, Gammaridea, Pictorial Key, Iberian Peninsula.

\section{Introducción}

Los anfípodos son un grupo de pequeños crustáceos que pueden vivir en hábitats muy diferentes. Pueden encontrarse en el mar, en el ambiente estuarino, en las aguas dulces, tanto epígeas como hipógeas, e incluso en ciertas zonas húmedas del ambiente terrestre, como por ejemplo en los invernaderos. Su distribución biogeográfica abarca desde las aguas frías polares hasta las de los trópicos. En cuanto a su distribución batimétrica, cabe señalar que se extienden desde el humus húmedo del suelo de algunos bosques hasta los grandes abismos marinos.

En la actualidad se conocen más de 6000 especies. Este elevado número de especies, unido a su pequeño tamaño y a su enorme abundancia en el ambiente acuático, ha motivado que frecuentemen-

* Centro de Investigaciones Marinas. Universidad de La Habana. Cuba. cim@comuh.uh.cu

** Dpto. Biol. Animal (Invertebrados). Facultad de Biología. Universidad de Barcelona. España. ajimeno@pie.xtec.es 
te, en los estudios ecológicos, aparezcan numerosos individuos pendientes de identificación. El interés actual por conocer la biodiversidad real de todas las regiones del mundo ha permitido abordar el estudio de grupos zoológicos que, como los anfípodos, pese a ser muy abundantes todavía son poco conocidos.

Los anfípodos europeos han sido objeto de numerosos estudios desde el siglo XIX. Cabe destacar los trabajos de Costa (1857), Della Valle (1893) y Mayer (1890). Entre los trabajos del siglo XX, los más notables son los de Stebbing (1906), los de Chevreux y Fage (1925) sobre la fauna de Francia, los de Lincoln (1979) sobre los anfípodos de la Gran Bretaña y los de Ruffo (editor) (1982, 1989,1993 y 1998) sobre los anfípodos del Mediterráneo. Cabe también citar una lista muy extensa de trabajos de Bellan-Santini, Diviacco, Kaim-Malka, Karaman, Krapp-Schickel, Ledoyer, Myers, Ruffo, Schiecke, Stock y otros. A pesar de lo anterior, sobre los anfípodos de la Península Ibérica, no habían aparecido más que algunos trabajos esporádicos hasta que empezaron a publicarse los trabajos de Bellan-Santini y Marques (1986), Marques y Bellan-Santini (1985, 1987, 1988, 1990a y 1990b) sobre los anfípodos marinos de Portugal, y los de Jimeno (1993), Jimeno y Turon (1995), Conradi et al. (1995) y Martí et al. (1995 y 1996) sobre los anfípodos mediterráneos ibéricos. Sobre los anfípodos dulceacuícolas, superficiales y subterráneos, cabe citar los trabajos de Notenboom $(1986,1987$ a y 1987b) en la península y los de Pretus (1990) y Jaume (1991) en las islas Baleares.

En 1991 Barnard y Karaman publicaron una guía para la identificación de la Familias y los Géneros de los anfípodos marinos del suborden Gammaroidea de todo el mundo. Es un trabajo muy completo y bien ilustrado pero, tal vez, demasiado amplio para ser utilizado por investigadores no especializados en este grupo. El objetivo de la presente clave ilustrada es precisamente ayudar a estas personas. Sólo abarca las especies de la Península Ibérica y, para que pueda ser útil a los no iniciados, está basada fundamentalmente en la comparación de imágenes. El único antecedente conocido de este tipo de guías ilustradas es la guía sobre la identificación de los gammáridos de Atlántico Occidental Tropical (Golfo de México y el Mar Caribe) de Ortiz (1994).

La ejecución de este trabajo ha sido posible, gracias a la financiación ofrecida por los Proyectos "Fauna Ibérica III" PB92-0121 y "Fauna Ibérica IV" PB95-0235, del Museo Nacional de Ciencias Naturales y del CSIC.

\section{Características y funcionamiento de la Guía}

Es una guía diseñada sobre la base de 2.023 figuras que representan individuos completos, en vista lateral, o estructuras corporales de interés para la identificación. La clave contiene 2 partes fundamentales:

Parte 1. Morfología. Es la parte introductoria inicial que trata los aspectos morfológicos generales y aquellos que son de interés para el diagnóstico. Está compuesta por las Figuras 1 a 10. Sus dibujos, que conviene conocer bien, permiten al lector familiarizarse con la gran variabilidad de formas que pueden presentar las piezas y las partes corporales de los anfípodos gammáridos. Es indispensable aprender las siglas que aparecen entre paréntesis, ya que sirven para identificar cada una de las piezas del cuerpo. Conviene que el usuario estudie bien este apartado. Luego es conveniente que realice la disección completa de algunos ejemplares, para así familiarizarse con la anatomía de los anfípodos, adquirir la técnica de la microdisección y desarrollar la habilidad necesaria en estos trabajos. Después se ha de estudiar, con sumo cuidado, las instrucciones para el manejo de la Guía que se explican a continuación.

Parte 2. Sistemática. Se inicia con la llamada "Clave Maestra", que es una clave que conduce a las Familias o a los diversos grupos de Familias. Está compuesta por las Láminas 1 a 10. Sigue una clave que permite confirmar las Familias y llegar a los Géneros. Está compuesta por las Láminas 11 a 76. Las Familias aparecen por orden alfabético y también los Géneros de cada Familia están dispuestos por orden alfabético. Se ha de advertir que como en la Clave Maestra, las Familias se agrupan en función de compartir un determinado carácter morfológico muy evidente, y como algunas presentan varios de estos caracteres, una misma Familia puede aparecer en varios grupos distintos. Es por ello que existe más de un camino para llegar a cada Familia.

Generalmente hay una lámina por cada Familia. Las Familias que comprenden muchos Géneros, como son las Familias Corophiidae-Ischyroceridae, Gammaridae-Melitidae y Lysianassidae, han precisado de 3 a 4 láminas. En la primera lámina de cada Familia, en el ángulo superior izquierdo, siempre se ofrece la información referente a la presencia o ausencia del flagelo accesorio, lo cual es muy útil para la identificación de los anfípodos. Las flechas que aparecen junto a las figuras, señalando algún punto de las mismas, indican características morfológicas fundamentales para su identificación que 
nunca deben ser obviadas. Los dibujos que carecen de números adjuntos, simplemente sirven para confirmar si el ejemplar a identificar pertenece o no a esa Familia. Los dibujos que llevan números adjuntos permiten, mediante la formación de secuencias de números, la identificación del Género al que pertenece el ejemplar a determinar. Cuando aparecen dos números entre paréntesis, por ejemplo (2-4), significa que la forma de esa pieza varía desde el dibujo 2 hasta el 4 . Se recomienda una cierta tolerancia en la selección del dibujo de los gnatópodos más parecidos a los del ejemplar que se está identificando, pues resulta imposible representar todas las variaciones existentes.

A las personas con poca experiencia se les recomienda que, para iniciarse en la identificación de los Géneros, seleccionen machos adultos, ya que éstos presentan rasgos más conspicuos. En ciertos casos, como por ejemplo en la Familia Ampeliscidae, las hembras son mucho más abundantes, por lo que no queda más remedio que utilizarlas.

La primera lámina de la Clave Maestra (Lámina 1) permite seleccionar, entre 9 grupos de Familias, a que grupo pertenece el ejemplar a identificar. Las nueve siguientes láminas de la Clave Maestra (Láminas 2 a 10), permiten seleccionar, entre 40 Familias diferentes, a que Familia pertenece el ejemplar a identificar. La siguiente parte de la clave, la parte de las Familias y Géneros (Láminas 11 a 76), permite confirmar la Familia y seleccionar, entre 142 Géneros, a que Género pertenece el ejemplar a identificar.

Si la Familia es monogenérica o tiene un solo Género representado en la Península Ibérica, la clave indica directamente el Género que es. Si la Familia está representada por más de un Género la clave ofrece una serie de dibujos numerados de diferentes partes del cuerpo. El procedimiento a seguir es ir escogiendo un número de cada uno de los grupos de piezas, de cada lámina, para conformar así una serie de números en orden creciente que se deberá comparar con las series numéricas que aparecen al pie de la última lámina de cada Familia. La serie que más se acerque a la obtenida por el usuario es la que indica el Género. Cuanto más Géneros comprende una Familia, más largas son las series numéricas, para garantizar así que, aunque se yerre en algún número, la selección sea correcta.

Cuando la experiencia permita reconocer directamente a que Familia pertenece un ejemplar, ya se podrá obviar la Clave Maestra inicial e ir directamente a discriminar el Género en las láminas posteriores (Láminas 11 a 76).
Esta clave está diseñada para identificar exclusivamente las especies de la Península Ibérica, por lo que utilizarla en otra región seguramente llevaría a identificaciones erróneas no imputables a los autores.

A continuación se presentan varios Ejemplos sobre el funcionamiento de la Guía:

Ejemplo 1. Se trata de un ejemplar que al consultar la primera lámina de la Clave Maestra (Lámina 1) se reconoce que sus coxas son atípicas, es decir son muy distintas de las coxas del gammárido típico que hay en el centro de la lámina. Se deberá pasar a la lámina del grupo de Familias con coxas atípicas (Lámina 3). En esta se puede observar que por la forma de sus coxas y de otras características pertenece a la Familia Iphimediidae, por lo que se pasará a la lámina 43, que es la primera de esta Familia. En primer lugar se ha de confirmar que las características del ejemplar coinciden con las características de esta familia. Luego se ha de proceder a buscar el Género, para lo cual se ha de ir seleccionando, en la lámina 44, si poseee o no flagelo accesorio (1 ó 2); el dorso más similar (3 ó 4); el maxilípedo (5 ó 6); el labio inferior (7 u 8); el gnatópodo 1 (9 ó 10); el gnatópodo 2 (11 ó 12); la mandíbula (13 ó 14) y el telson (15 ó 16). Supongamos que los caracteres elegidos han sido: $2,3,5,8,10,12,13$ y 16. Como esa serie coincide con la de Epimeria; ése es el Género del ejemplar que se está identificando.

Si las características del ejemplar no hubieran coincidido con ninguna de las Familias que aparecen citadas en la Lámina 3, se debe retroceder a la Lámina 1 y buscar en ella otro grupo de Familias con las que presente alguna otra coincidencia.

Ejemplo 2. Se trata de un ejemplar que al consultar la primera lámina de la Clave Maestra (Lámina 1) se reconoce que su telson es distinto al del gammárido típico que hay en el centro de la lámina. Se deberá pasar a la lámina del grupo de Familias con telson grueso y blando (Lámina 5). En ésta se puede observar que, por la forma de las coxas y de los gnatópodos, pertenece a la Familia Corophiidae-Ischyroceridae, por lo que se pasará a observar las láminas 22 y 23 . Una vez confirmado que pertenece a esa Familia se procederá a consultar las láminas 24 y 25 para seleccionar el Género. Se ha de escoger el tipo de coxa (1 ó 2); el tipo de flagelo accesorio (3-9); el tipo de palpo mandibular (1024); el tipo de cabeza (25-42); el tipo de gnatópodo 1 (43-60); el tipo de gnatópodo 2 (61-78); el tipo de urópodo 3 y de telson (79-108). Supongamos que los caracteres elegidos han sido: $\mathbf{2 , 3}, \mathbf{1 7}, \mathbf{3 7}, \mathbf{5 0}, \mathbf{6 7}$ 
y 79. Como esa serie coincide con la de Corophium; ése es el Género del ejemplar que se está identificando.

Si la serie de los caracteres elegidos no coincide con ninguna de las que corresponden a los distintos Géneros, seguramente se ha realizado alguna elección incorrecta, ya sea a nivel de Géneros o a nivel de Familia, por lo que se deberá volver a la lámina de la Familia o a la lámina inicial (Lámina 1) respectivamente.

\section{Sistemática}

Las 40 Familias y los 142 Géneros encontrados aparecen en las siguientes láminas

\begin{tabular}{lc}
\hline Familias y Géneros & Lám \\
\hline AMPELISCIDAE (Ampelisca, Bybilis y Haploops) & 11 \\
AMPHILOCHIDAE & 13 \\
(Amphilochoides, Amphilochus, Gitana y Paramphilochoides) & \\
AMPITHOIDAE & 15 \\
(Ampithoe, Amphitolina, Peramphithoe) & \\
ARGISSIDAE (Argissa) & 17 \\
BIANCOLINIDAE (Biancolina) & 18 \\
BOGIDIELLIDAE (Bogidiella) & 19 \\
CHELURIDAE (Chelura) & 20 \\
COLOMASTIGIDAE (Colomastix) & 21 \\
COROPHIDAE-ISCHYROCERIDAE & 22
\end{tabular}

(Aora, Bonnierella, Cerapopsis, Corophium, Ericthonius, Gammaropsis, Ischyrocerus, Jassa, Lembos (sensu lato), Leptocheirus, Microdeutopus, Microjassa, Neohela, Parajassa, Photis, Protomedeia, Siphonoecetes,

Microprotopus y Unciola)

CRANGONYCTIDAE (Crangonyx) 26

CRESSIDAE (Cressa)

CYPROIDEIDAE (Peltocoxa)

DEXAMINIDAE

(Atylus, Guernea, Dexamine y Tritaeta)

EUSIRIDAE

(Apherusa, Eusirella, Eusiroides, Eusiropsis, Eusirus,

Rhachotropis y Stenopleura)

GAMMARELLIDAE (Gammarellus)

GAMMARIDAE-MELITIDAE

(Abludomelita, Bathyceradocus, Cheirocratus, Dulichiella,

Dulzura, Echinogammarus, Elasmopus, Gammaracanthus,

Gammarus, Haploginglymus, Liagoceradocus, Lusigammarus,

Maera, Maerella, Melita, Metahadzia, Metaniphargus,

Niphargus, Parapseudoniphargus, Psammogammarus,

Pseudoniphargus y Riphidogammarus)

HAUSTORIIDAE (Bathyporeia y Haustorius)

HYALIDAE (Hyale, Micropythia y Parhyale)

HYPERIOPSIDAE (Parargissa)

IPHIMEDIIDAE (Iphimedia y Epimeria)

LEUCOTHOIDAE (Leucothoe)

LILJEBORGIIDAE (Listriella, Liljeborgia y Sextonia)
LYSIANASSIDAE

48

(Acidostoma, Ambasia,Bathyamaryllis, Crybelocephalus,

Cyphocaris, Hippomedon, Ichnopus, Lepidepecrum,

Lysianassa, Lysianassina, Onisimus, Orchomene,

Paracallisoma, Paracentromedon, Paralicella,

Podoprion, Schisturella, Scopelocheirus, Socarnes,

Socarnopsis, Tmetonyx y Tryphosites)

MEGALUROPIDAE (Megaluropus)

MELPHIDIPPIDAE (Melphidippella)

METACRANGONYCTIDAE (Metacrangonyx)

NUUANIDAE (Gammarella)

OEDICEROTIDAE

(Monoculodes, Oediceros, Perioculodes, Pontocrates

y Synchelidium)

PARDALISCIDAE

(Halice, Halicoides, Nicippe y Spelaeonicippe)

PHLIANTIDAE (Pereionotus)

PHOXOCEPHALIDAE

(Harpinia, Metaphoxus y Paraphoxus)

PLEUSTIDAE (Parapleustes, Pleusymtes y Stenopleustes) 63

PODOCERIDAE (Podocerus)

SALENTINELLIDAE (Salentinella)

SEBIDAE (Seba)

STEGOCEPHALIDAE

(Parandania, Parandaniexis y Stegocephaloides)

STENOTHOIDAE

(Hardametopa, ParametopaProboloides y Stenothoe)

SYNOPIIDAE (Syrrhoe)

TALITRIDAE (Orchestia, Taliltrus y Talorchestia)

UROTHOIDAE (Urothoe)

INCERTAE SEDIS (Sensonator)

\section{Bibliografía}

BARNARD, J.L. y KARAMAN, G., 1991. The families and genera of Marine Gammaridean Amphipoda (except marine Gammaroids). Records of the Australian Museum, Suppl., 13(1-2): 1-866.

Bellan-Santini, D. y Marques, J.C., 1984. Contribution à l'étude des amphipodes des costes du Portugal. Ciência Biológica. Monografia, 5: 131-149

Chevreux, E. y Fage, L., 1925. Amphipodes. En: Faune de France, vol. 9. Paul Lechevalier. Paris. 488 pp.

Conradi, M., LóPez-GonzÁlez, P.J. et Bellan-SANTini, D., 1995. A new species of Urothoe (Amphipoda, Gammaridea) from the Iberian Peninsula. Cahiers de Biologie Marine, 36: 9-13

Costa, A,. 1857. Richerche sui Crostacei Amfipodi del golfo di Napopli. Memorie della Reale Accademia delle Scienze, Napoli, 1: 165-335.

Della Valle, A., 1893. Gammarini del Golfo di Napoli. Fauna und Flora des Golfes von Neapel und der angrenzenden Meeres-Abschnitte, 20: 1-948.

Jaume, D., 1991. Two new species of the amphipod genus Pseudoniphargus (Crustacea) from Cabrera (Balearic Islands). Stygologia, 6(3): 177-189. 
JiMENO, A., 1993. Los anfípodos asociados a algas y a bivalvos en el litoral catalán. Publicaciones Especiales del Instituto Español de Oceanografía, 11: 169-174.

Jimeno, A y TuRón, X., 1995. Gammaridea and Caprellidea of the northeast coast of Spain: ecological distribution on diferent types of substrata. Polskie Archiwum Hidrobiologii, 42(4): 495-516.

LinColn, R.J., 1979. British Marine Amphipoda: Gammaridea. British Museum (Natural History). London. 658 pp.

Marques, J.C. y Bellan-Santini, D., 1985. Contribution à l'étude systématique et écologique des amphipodes (Crustacea, Amphipoda) des côtes $\mathrm{du}$ Portugal. Premier inventaire des espèces (Gammariens et Caprelliens). Ciência Biológica. Ecologia e Sistemática, 5: 299-353.

Marques, J.C. y Bellan-Santini, D., 1987. Crustacea Amphipodes des côtes du Portugal: faune de estuaire du Mira (Alenteja, cote sud-ouest). Cahiers de Biologie Marine, 28: 465-480.

Marques, J.C. y Bellan-Santini, D., 1988. Crustacea Amphipodes des côtes du Portugal: faune du canal de Santo Andre. Anais da Faculdade de Ciências do Porto, 67: 219-243.

Marques, J.C. y Bellan-Santini, D., 1990a. Benthic amphipod fauna (Crustacea) of the Portuguese coast. Biogeographical considerations. Marine Nature, 3: 43-51.

Marques, J.C. y Bellan-Santini, D., 1990b. Faune d'amphipodes des milieus lagunaires du Portugal. Actualisation des dones et comparaison avec d'autre systèmes Atlantiques et Méditerranéens. Mésogée (Marseille), 50: 53-61.

Martí, A. y Villora-Moreno, S., 1996a. A new interstitial species of Gammarella (Amphipoda, Gammaridea) from the western Mediterranean Sea. Contributions to Zoology, 65(2): 1-9.

Martí, A. y Villora-Moreno, S., 1996b. Eriopisella ruffoi, new species (Amphipoda: Gammaridea), interstitial, first record of Eriopisella from the Mediterranean Sea. Journal of Crustacean Biology, 16(3): 613-625.

Mayer, R., 1890. Die Caprelliden des Golfes von Neapel und der angrenzendenn Meeres-Abschnitte. Nach-trag zur Monographie derselben. Fauna und Flora des Golfes von Neapel und der angrenzenden Meeres-Abschnitte, 17: 1-157.

Notenboom, J., 1986. The species of the genus Pseudoniphargus Chevreux, 1901 (Amphipoda) from northern Spain. Bijdragen tot de Dierkunde, 56: 75-122.

Notenвoom, J., 1987a. Species of the genus Pseudoniphargus Chevreux, 1901 (Amphipoda) from the Betic cordillera of southern Spain. Bijdragen tot de Dierkunde, 57: 87-150.
Notenboom, J., 1987b. Lusitanian species of the amphipod genus Pseudoniphargus Chevreux, 1901 (Amphipoda) with a key to all known Iberian species. Bijdragen tot de Dierkunde, 57: 191-206.

OrTIZ, M., 1994. Clave gráfica para la identificación de familias y géneros de anfípodos del Suborden Gammaridea del Atlántico Occidental Tropical. Anales del Instituto de Investigaciones Marinas de Punta de Betín, 23: 59-101.

Pretus, J.L., 1990. Three new species of the genus Pseudoniphargus (Crustacea, Amphipoda) in Balearic ground waters. Stygologia, 5: 101-118.

Ruffo, S. (Editor). 1982. The Amphipoda of the Mediterranean. Part 1. Gammaridea (Acanthonozomatidae to Gammaridae) Mémoires de l'Institut Océanographique. Fondation Albert I'r, Prince de Monaco, 13: 1-364.

Ruffo, S. (Editor). 1989. The Amphipoda of the Mediterranean. Part 2. Gammaridea (Haustoriidae to Lysianassidae). Mémoires de l'Institut Océanographique. Fondation Albert I ${ }^{e r}$, Prince de Monaco, 13: 365-576.

Ruffo, S. (Editor). 1993. The Amphipoda of the Mediterranean. Part 3. Gammaridea (Melphidippidae to Talitridae), Ingolfiellidea, Caprellidea. Mémoires de l'Institut Océanographique. Fondation Albert I'r, Prince de Monaco, 13: 576-813.

Ruffo, S. (Editor). 1998. The Amphipoda of the Mediterranean. Part 4. Localities. Key to Families. Ecology. Faunistics and Zoogeography. Bibliography. Mémoires de l'Institut Océanographique. Fondation Albert ${ }^{2}$, Prince de Monaco, 13: 814-959

StebBing, T.R.R., 1906. Amphipoda I. Gammaridea. Das Thierreich, 21: 1-806. 


\section{Parte I. MORFOLOGÍA}

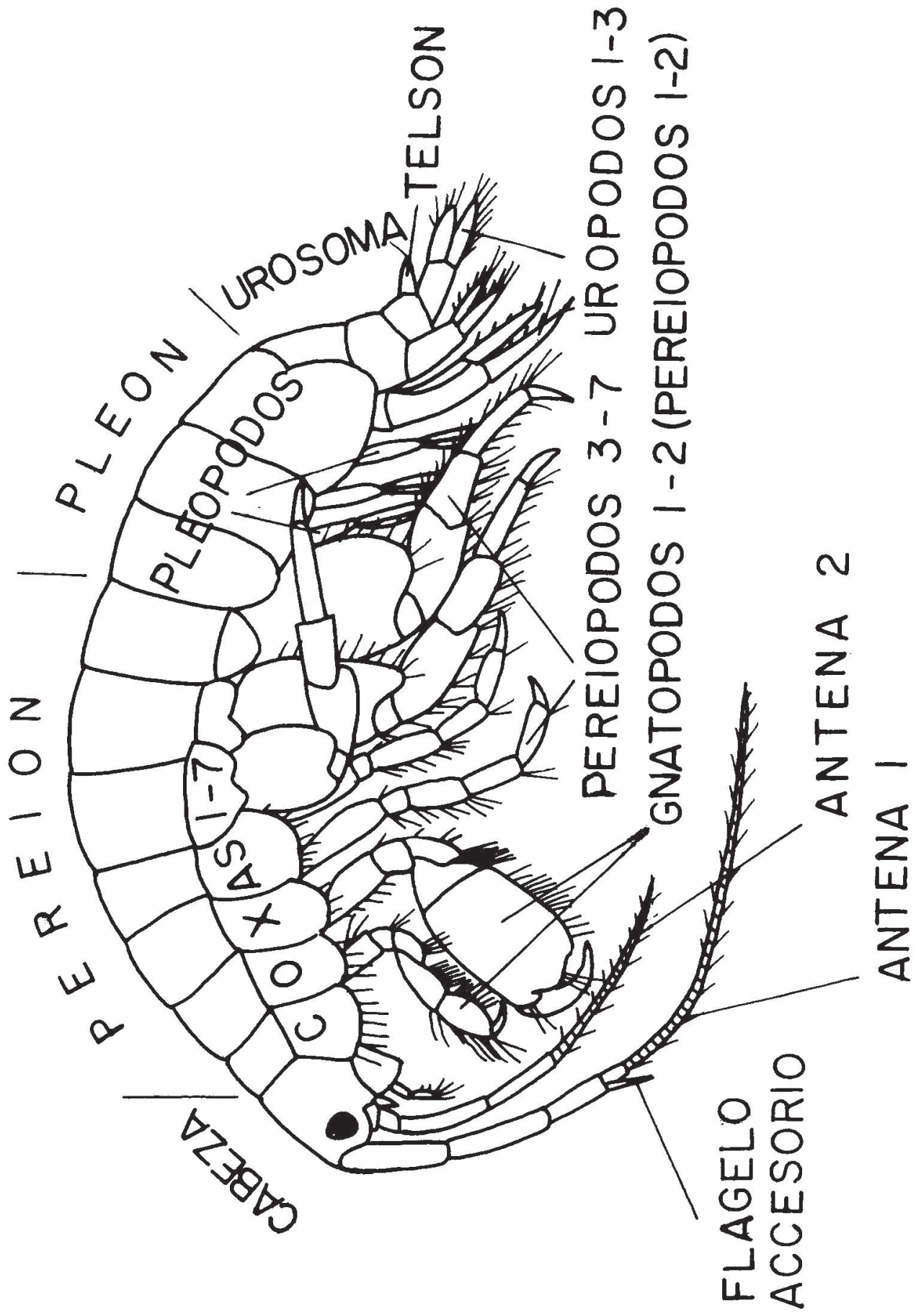

Fig. 1.- Morfología externa de un anfípodo del suborden Gammaridea. (C, cabeza; A1, antena 1; A2, antena 2; G1, gnatópodo 1; G2, gnatópodo 2; P3 - P7, pereiópodos 3 a 7; U1- U3, urópodos 1 a 3; PLP1-3, pleópodos 1 a 3; T, telson). 


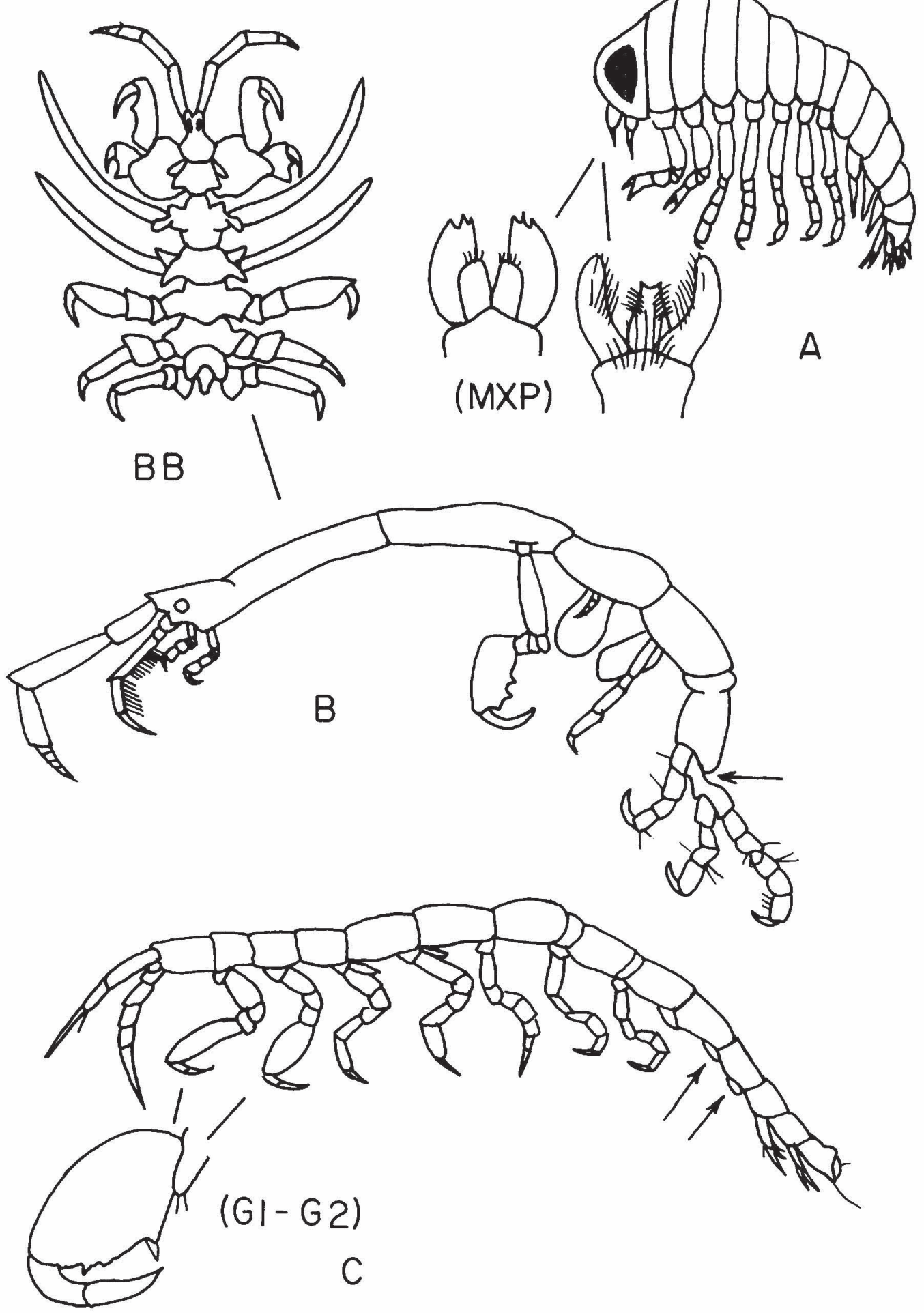

Fig. 2.- Morfología externa de los anfípodos de los subordenes restantes: A, Suborden Hyperiidea; B, Suborden Caprellidea; BB, Familia Cyamidae; C, Suborden Ingolfiellidea. (MXP, maxilípedo; G1-2, gnatópodos 1 y 2). 


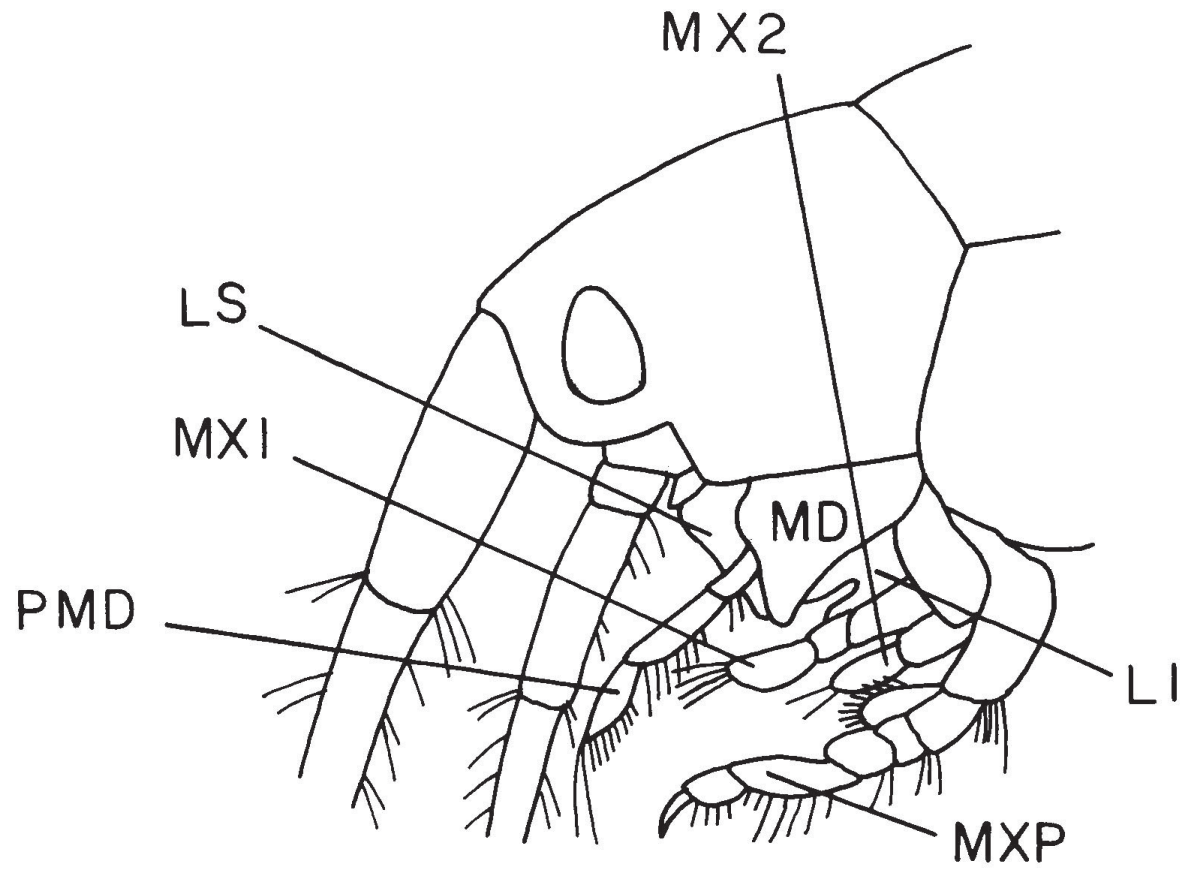

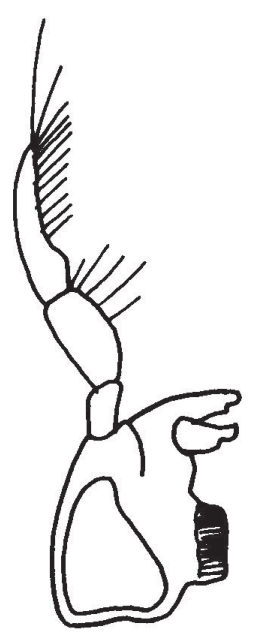

MD

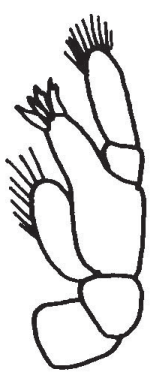

$\mathrm{MXI}$

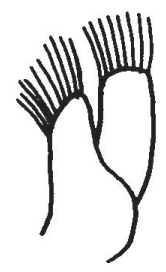

$\mathrm{MX} 2$

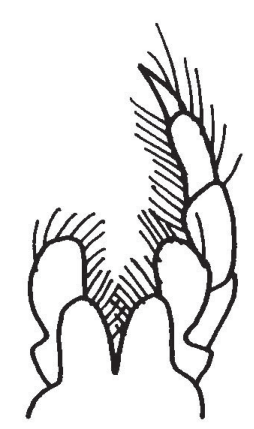

$\mathrm{MXP}$

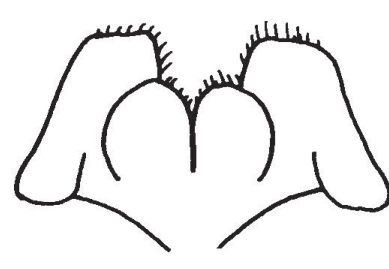

LI

Fig. 3.- Arriba. Cabeza de un anfípodo del Suborden Gammaridea, en visión lateral, mostrando las piezas bucales. (MD, mandíbula; LS, labio superior; PMD, palpo mandibular; MX1, maxila 1; MX2, maxila 2; MXP, maxilípedo; LI, labio inferior). Abajo. Piezas bucales separadas. (MD, mandíbula; MX1, maxila 1; MX2, maxila 2; MXP, maxilípedo; LI, labio inferior). 

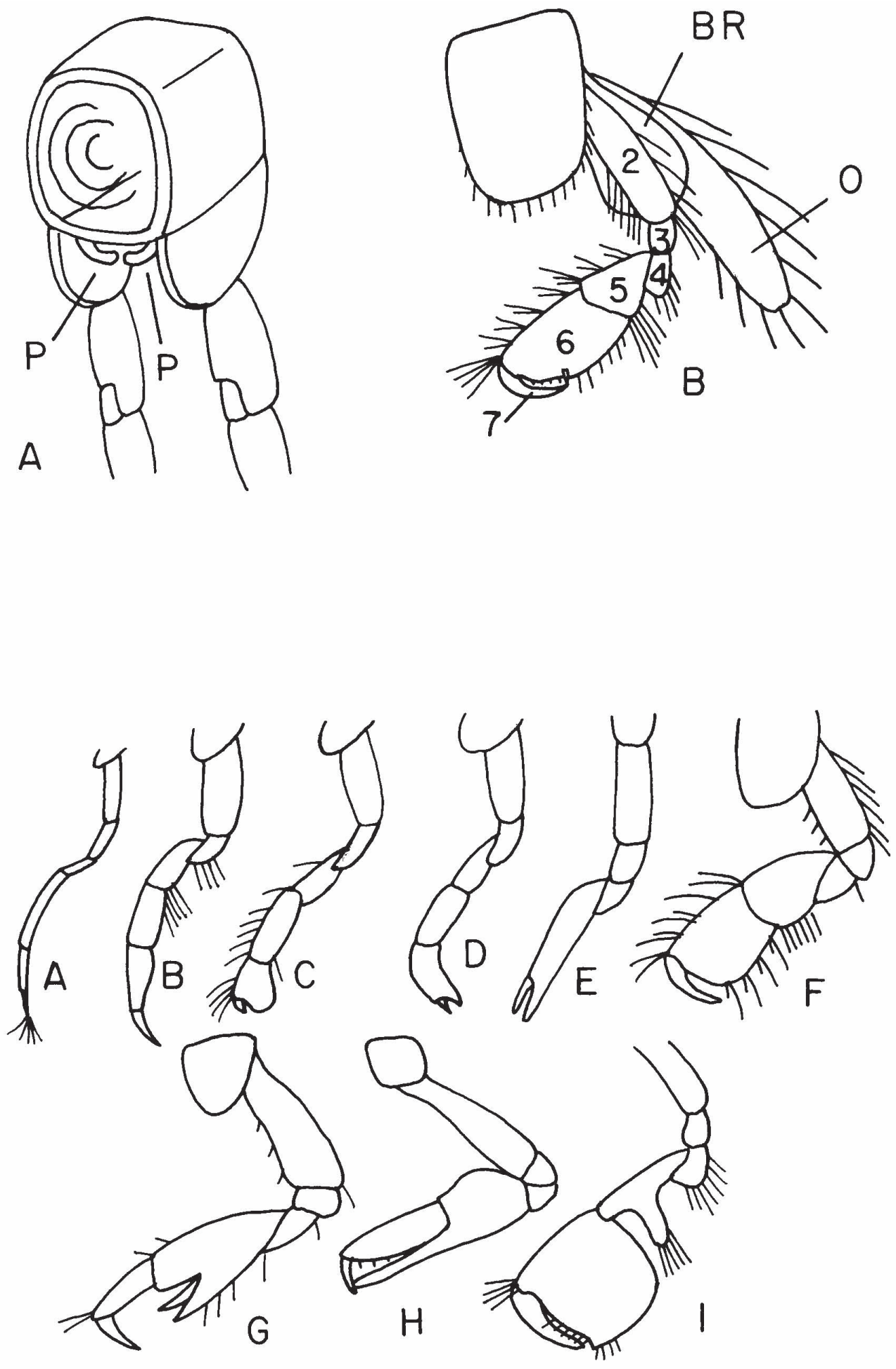

Fig. 4.- Arriba. Dimorfismo sexual. (A, séptimo segmento pereional de un macho; P, pene; B, gnatópodo 2 de una hembra; O, oostegito; BR, branquia). Artejos de un gnatópodo. (2, basipodito; 3 , isquiopodito, 4, meropodito; 5 , carpopodito; 6 , protopodito; 7, dactilopodito). Abajo. Tipos de gnatópodos. (A, filiforme; B, simple; C, débilmente subquelado; D, débilmente quelado; E, fuertemente quelado; F, subquelado; G y H, carpoquelado; I, eusiroideo. 


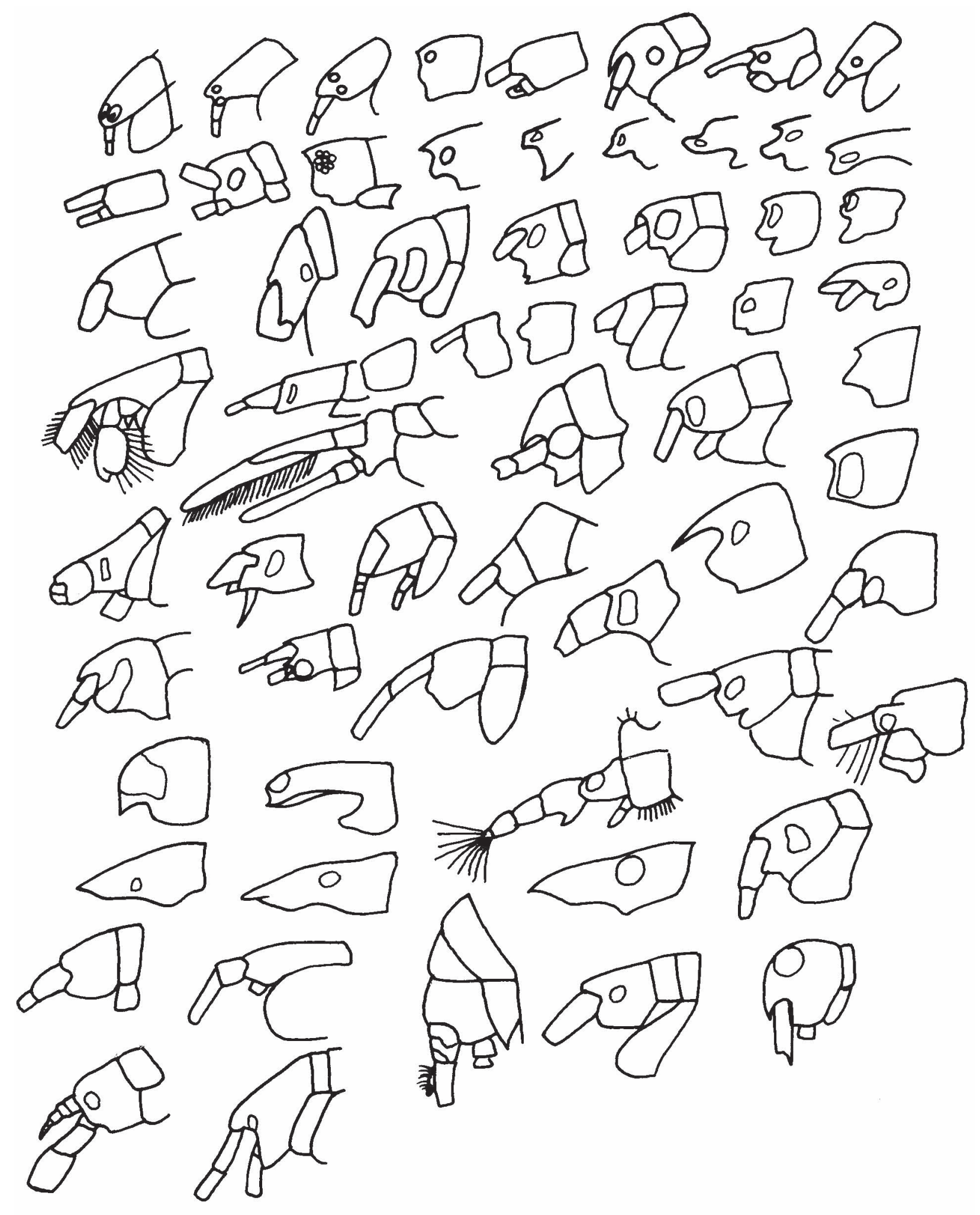

Fig. 5.- Tipos de cabeza en visión lateral. 


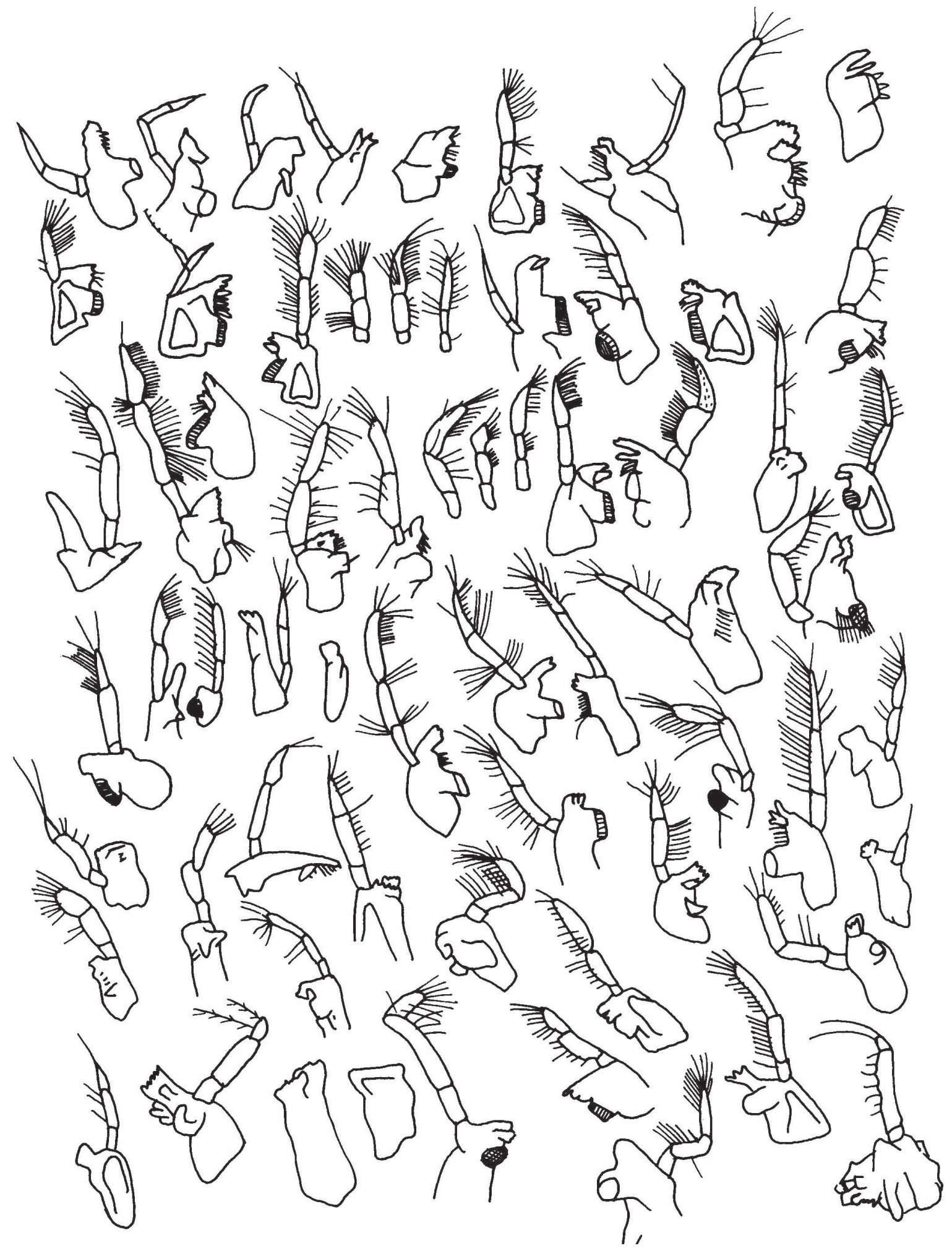

Fig. 6.- Tipos de mandíbulas. 

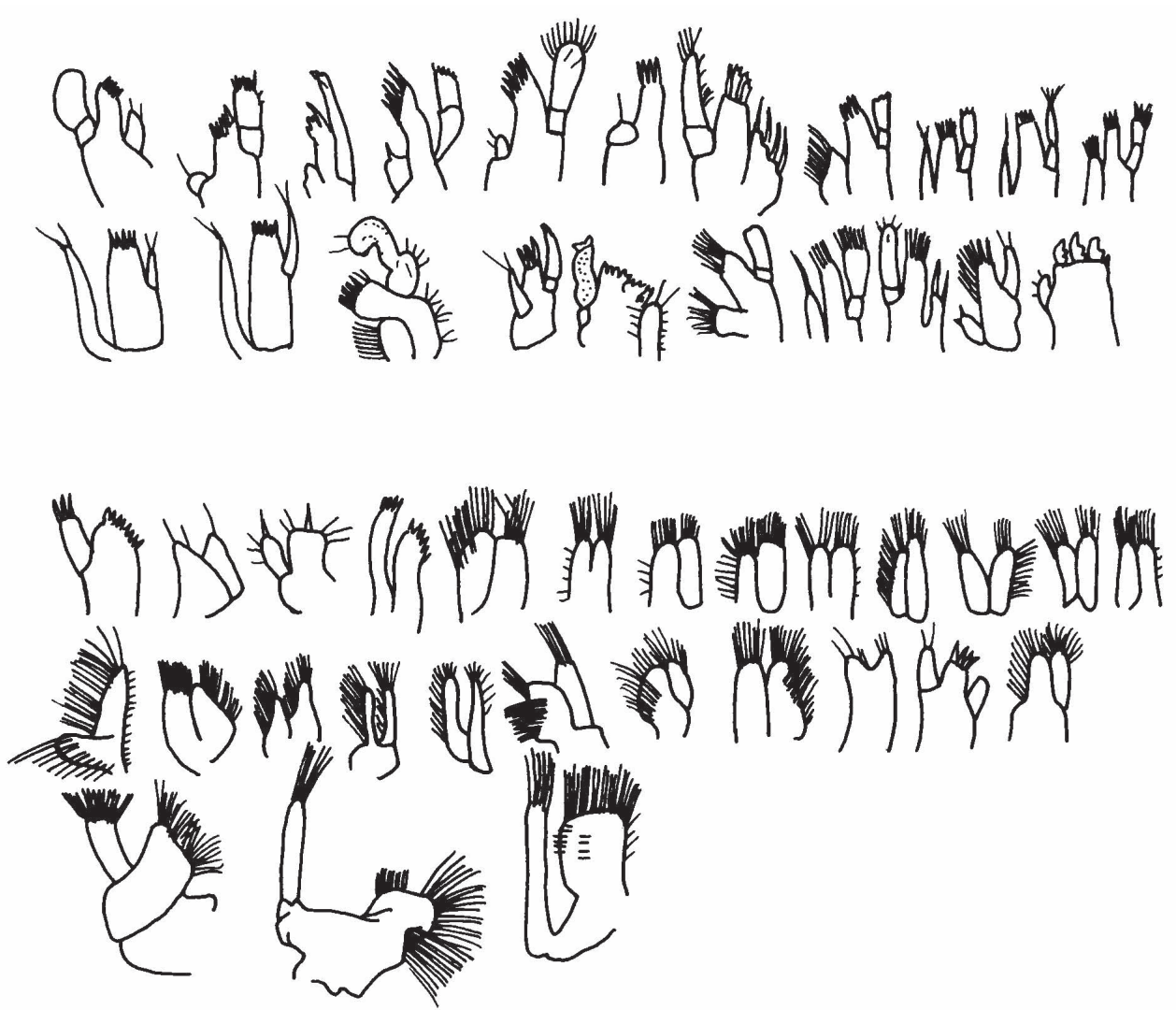

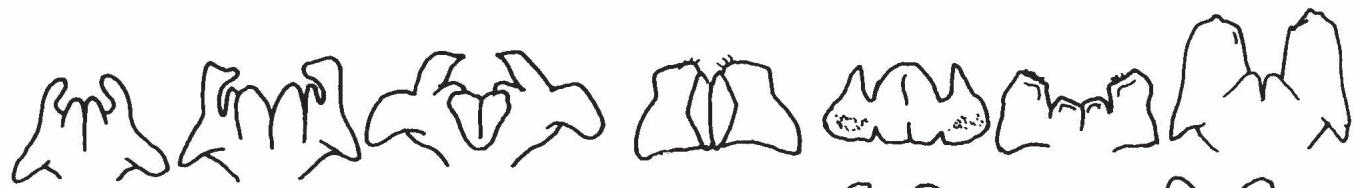

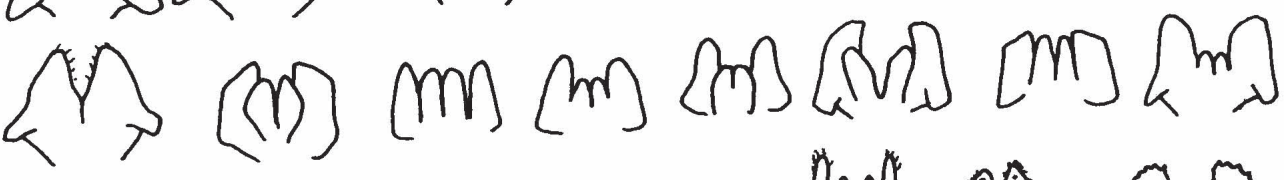

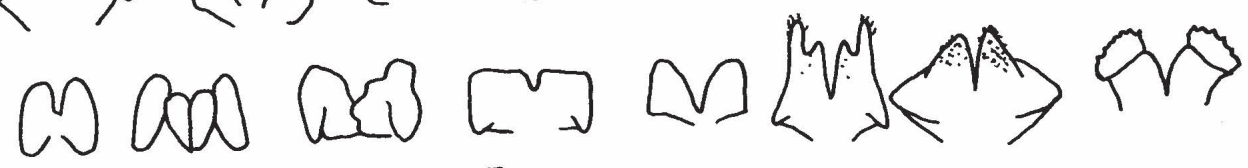

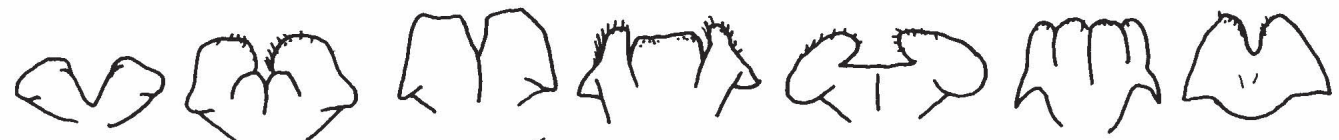
Con

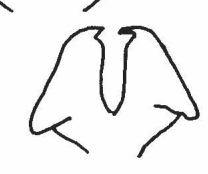

m
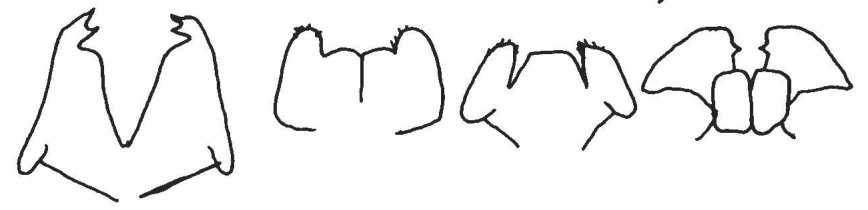

Fig. 7.- Arriba. Tipos de maxilas 1 (primeras maxilas). En medio. Tipos de maxilas 2 (segundas maxilas). Abajo. Tipos de labios inferiores. 

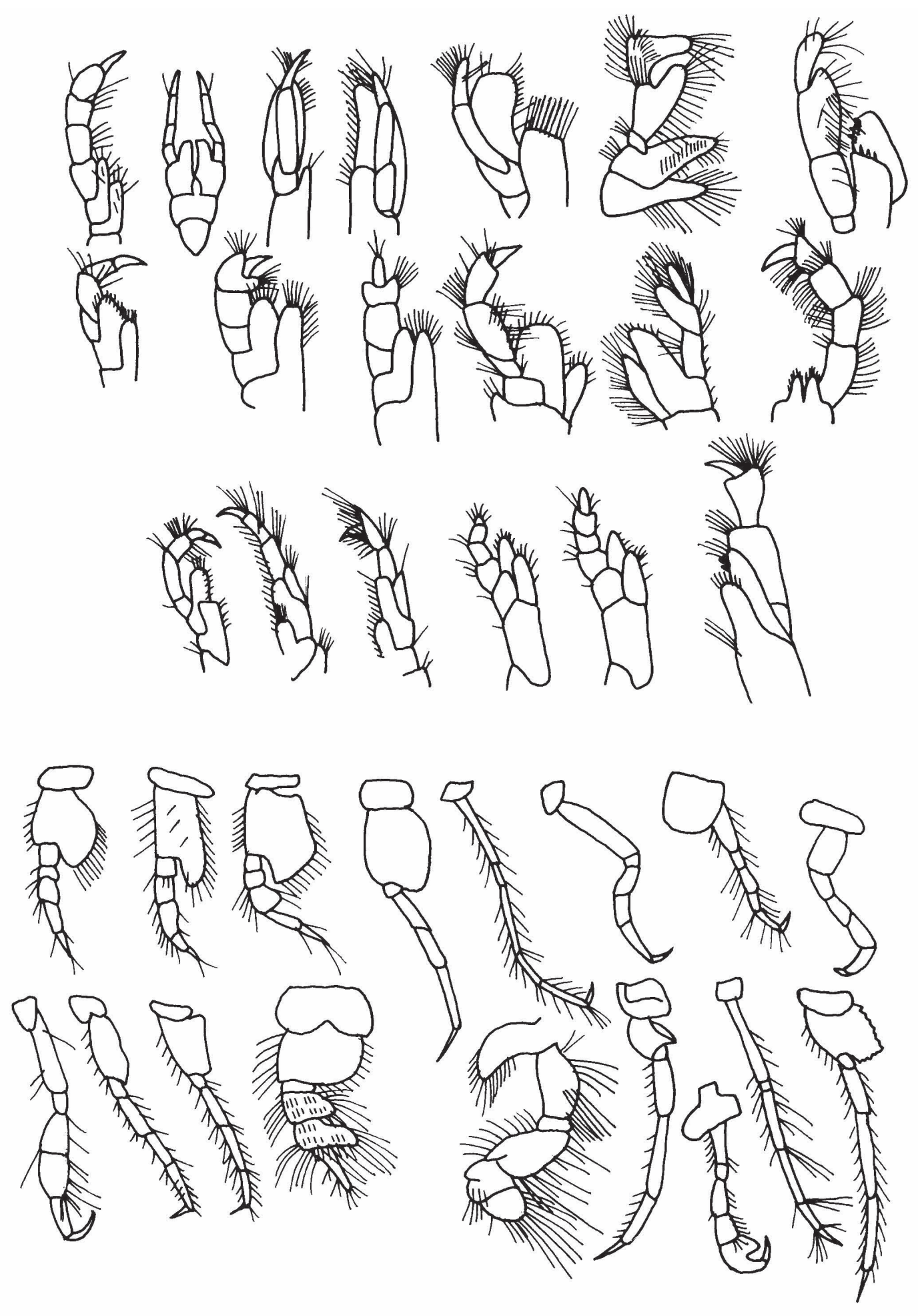

Fig. 8.- Arriba. Tipos de maxilípedos. Abajo. Tipos de pereiópodos. 

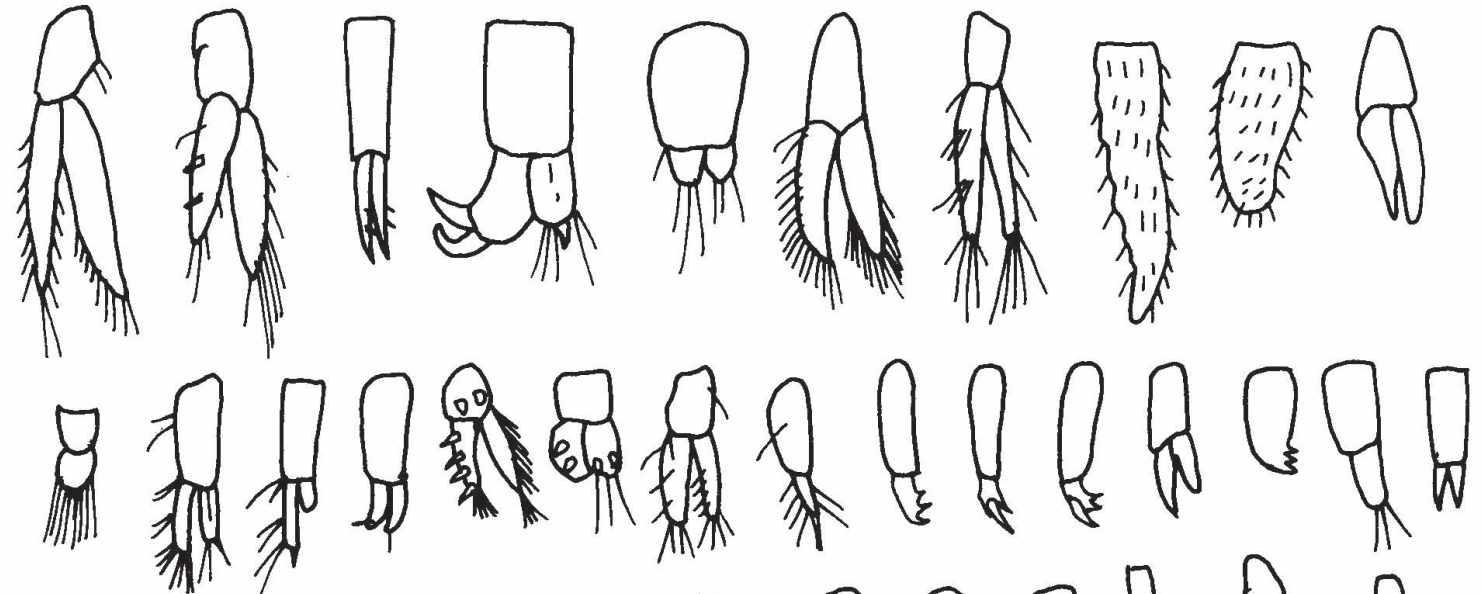

梨
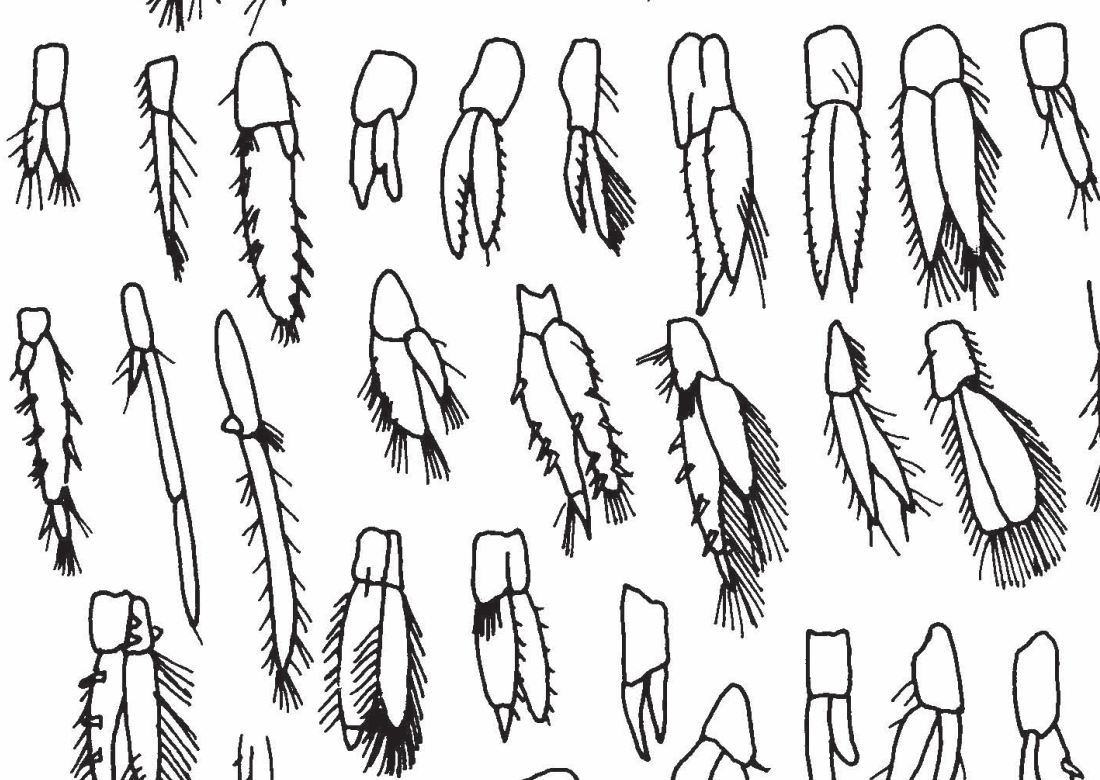

低
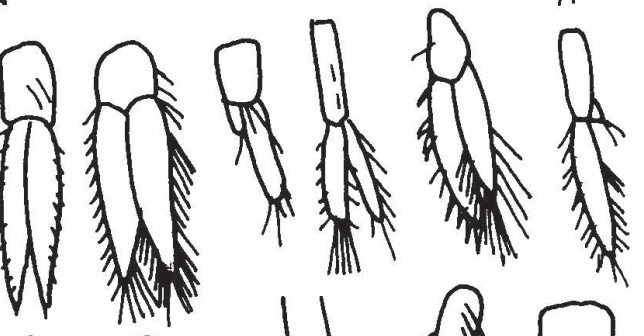

得

$\sqrt{5}$
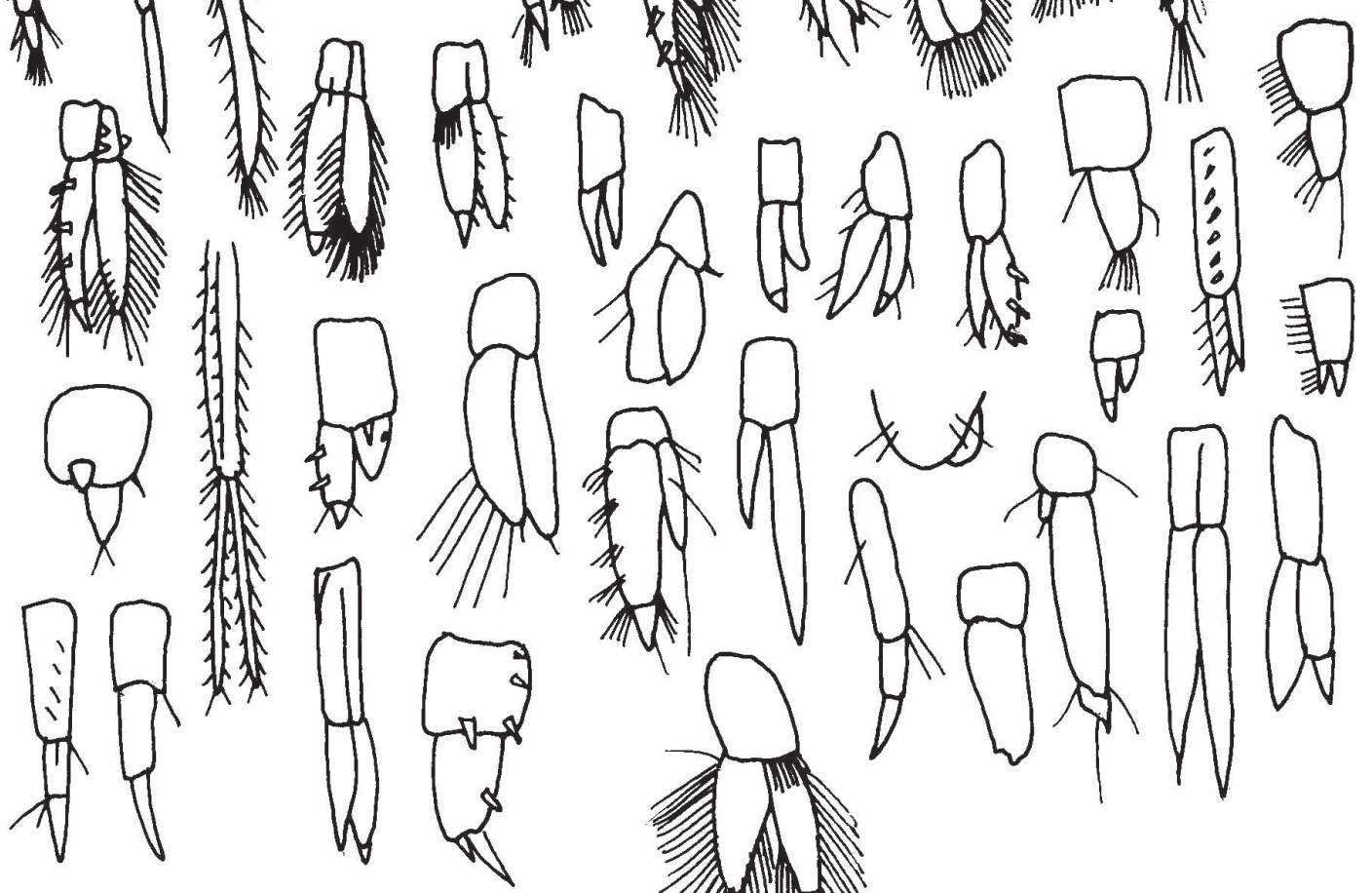

美

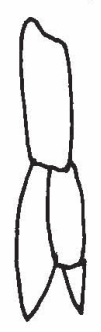

Fig. 9.- Tipos de urópodos 3. 


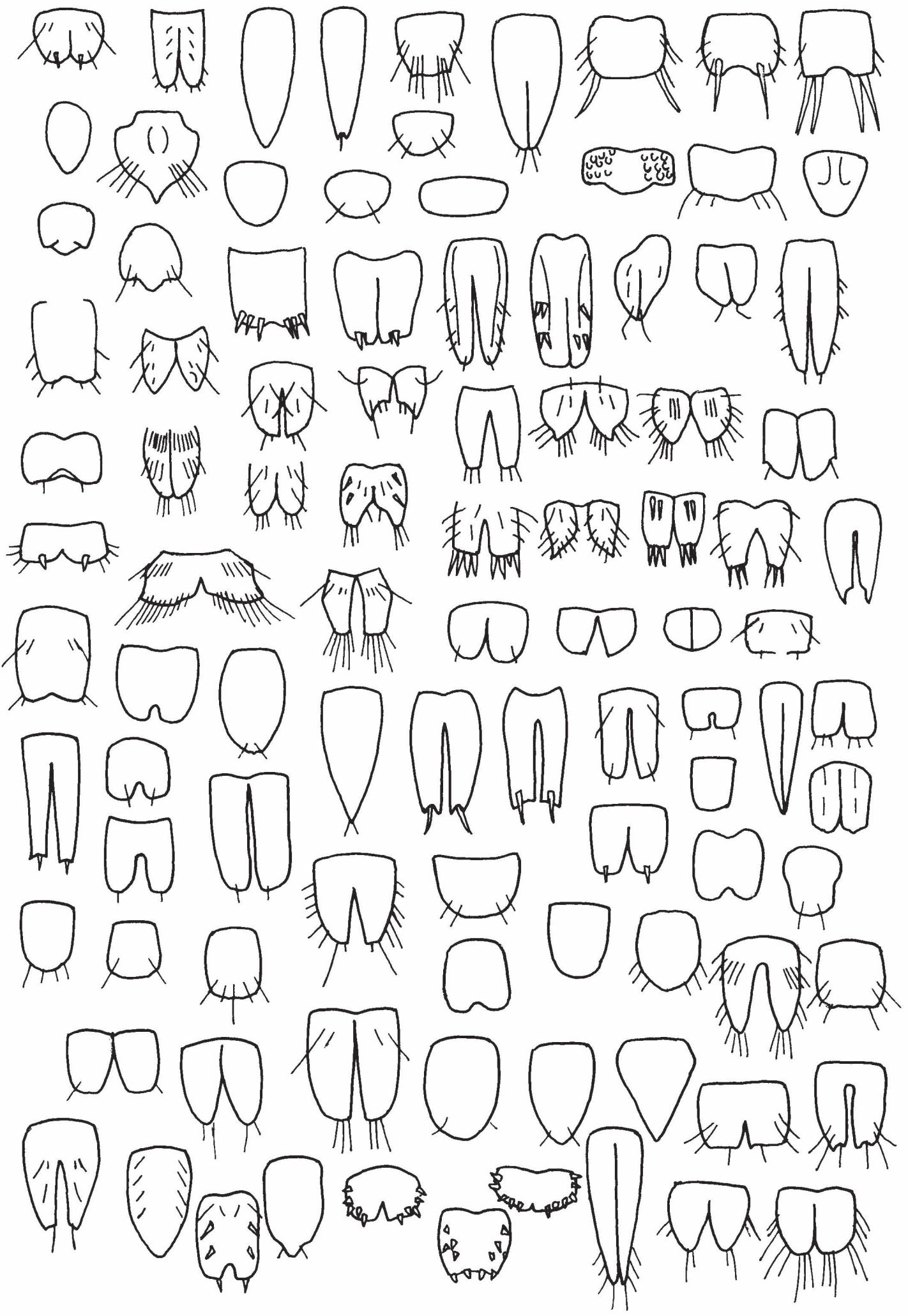

Fig. 10.- Tipos de télsones. 


\section{Parte II SISTEMÁTICA}

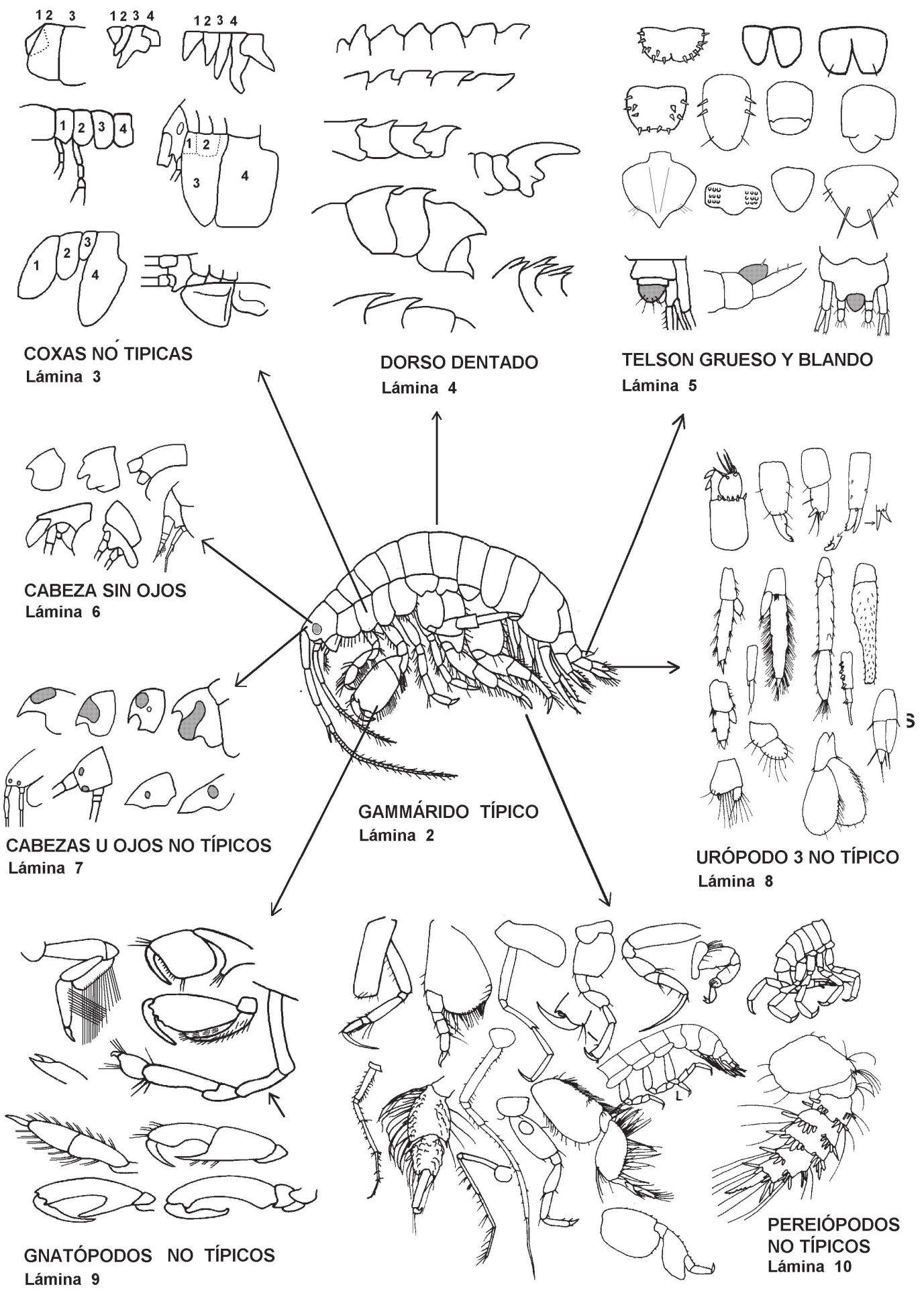

Lámina 1.- Clave Maestra en la que se presenta un gammárido típico del cual se han diferenciado, artificialmente, ocho grupos de Familias con caracteres morfológicos atípicos. 

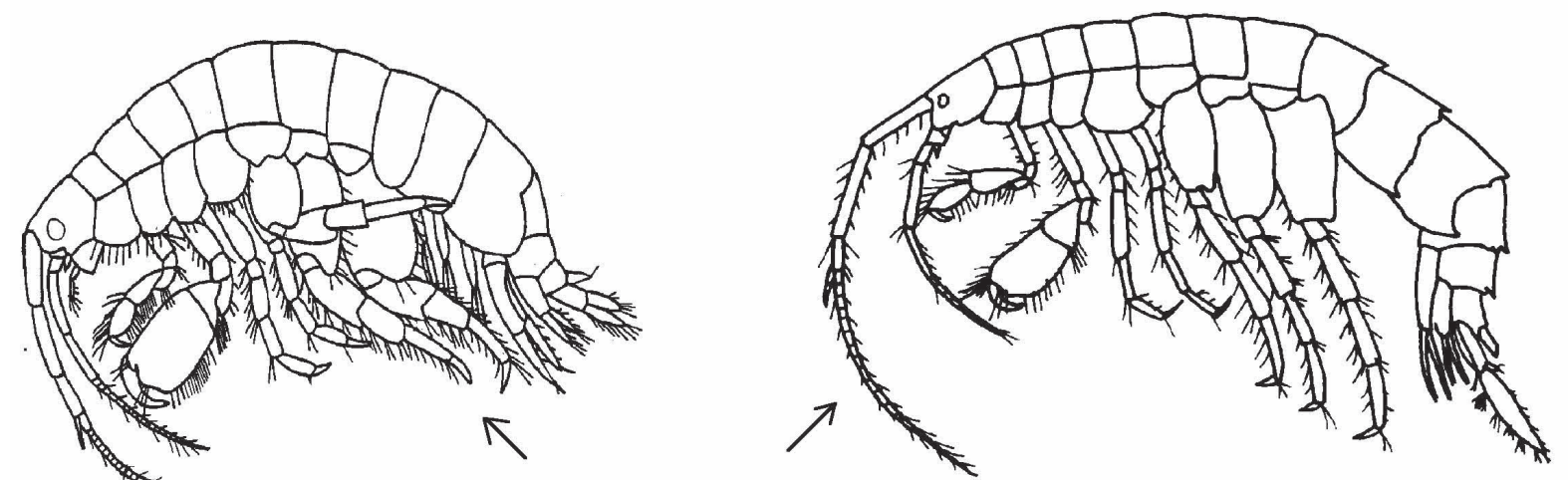

F. GAMMARIDAE - MELITIDAE

Láminas 34 a 37
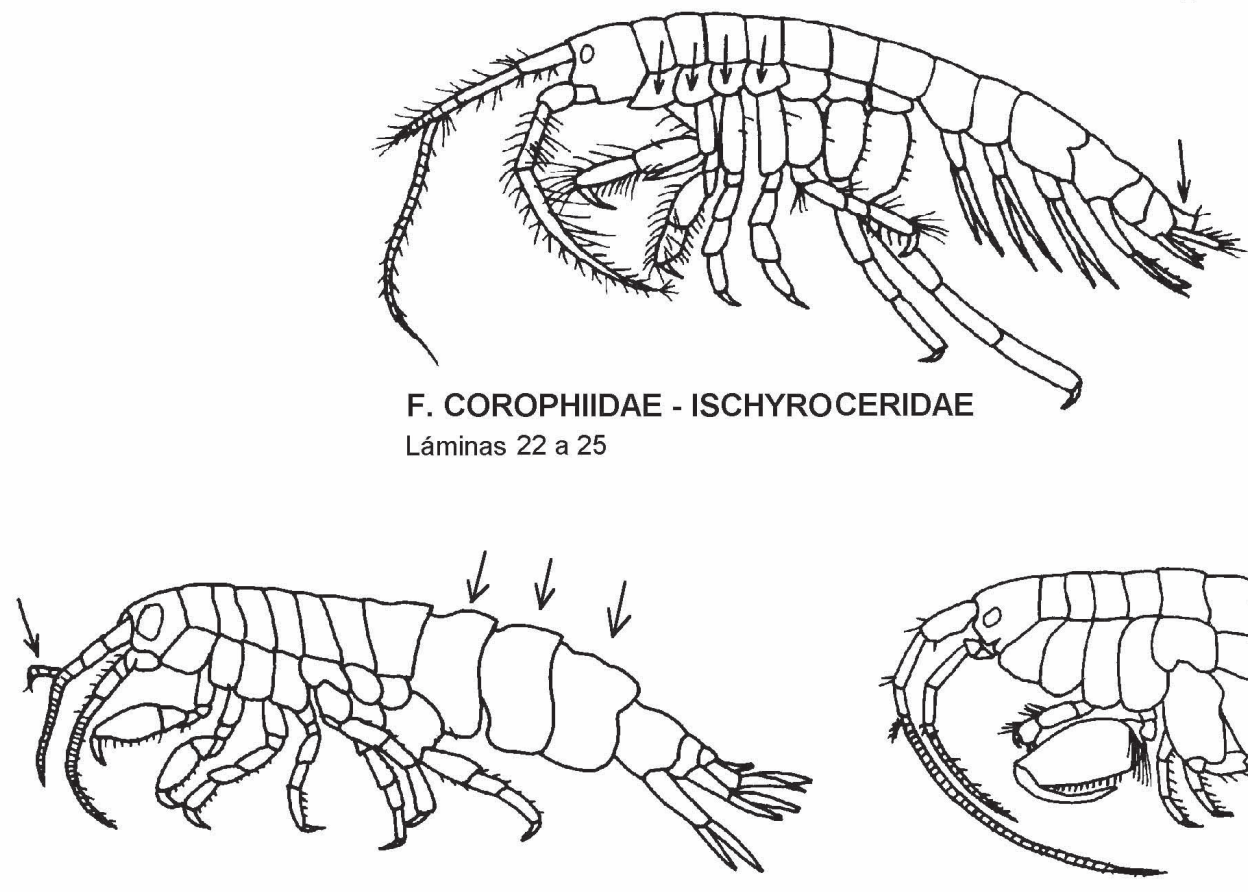

F. GAMMARELLIDAE

Lámina 33

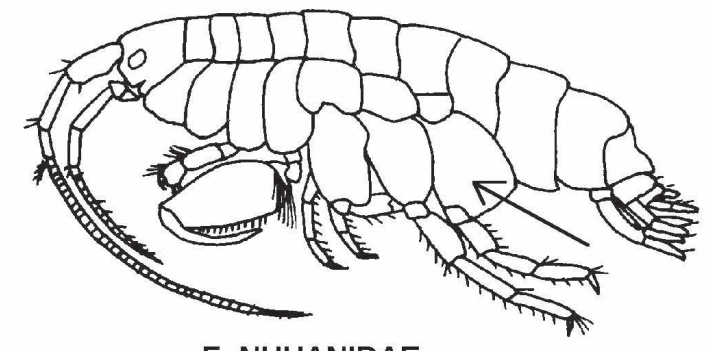

F. NUUANIDAE

Lámina 55

Lámina 2.- Grupo de Familias con caracteres típicos. 

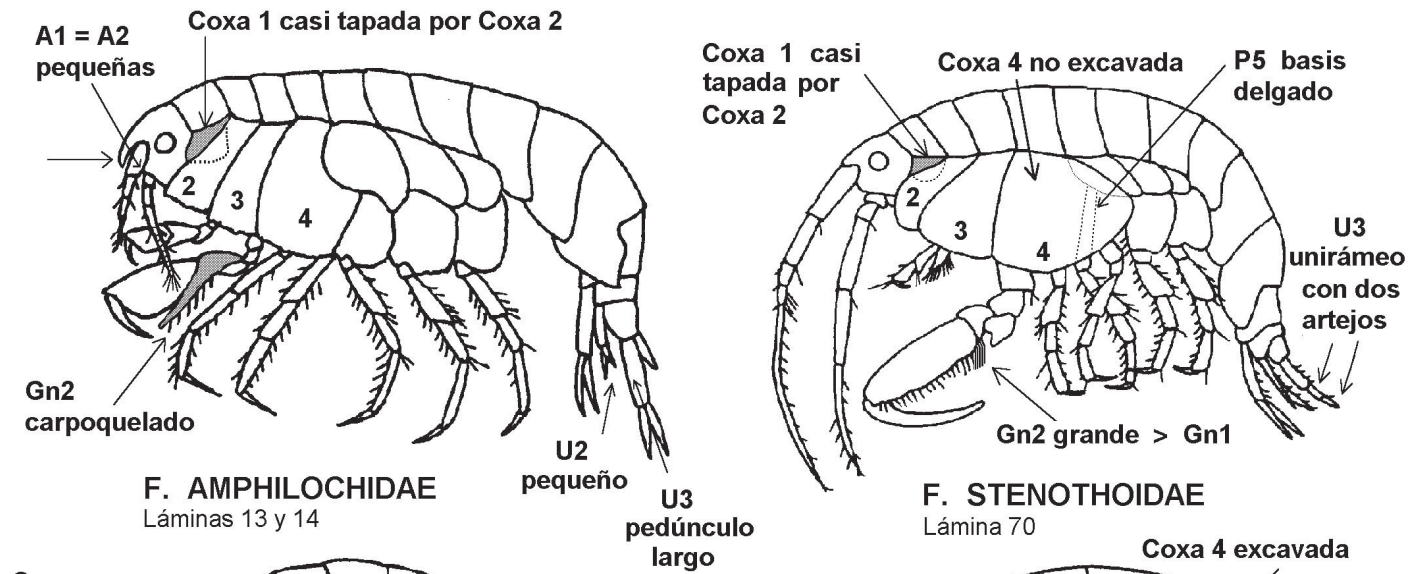

\section{F. STENOTHOIDAE}

Lámina 70

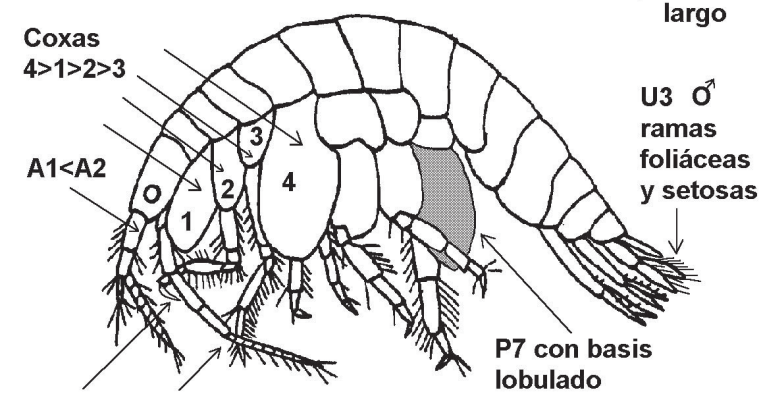

Gn1 - Gn2 lobulado

\section{no quelados \\ F. ARGISSIDAE}

Lámina 17

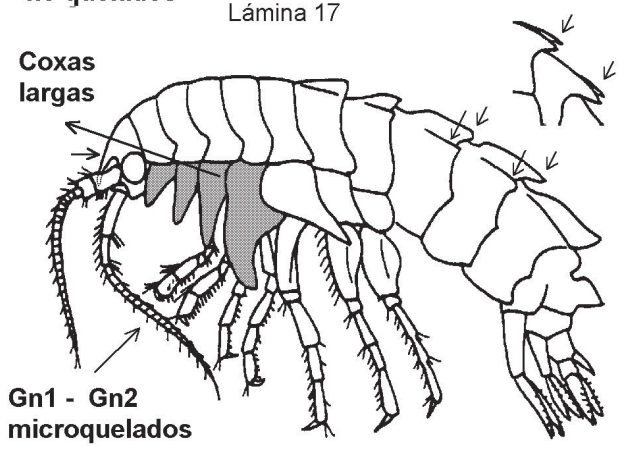

F. IPHIMEDIIDAE

Láminas 43 y 44

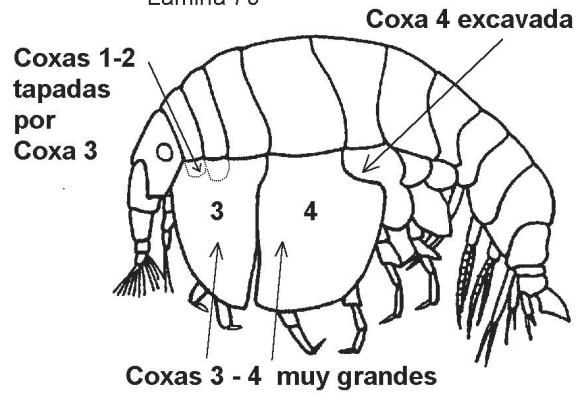

F. CYPROIDEIDAE

Lámina 28

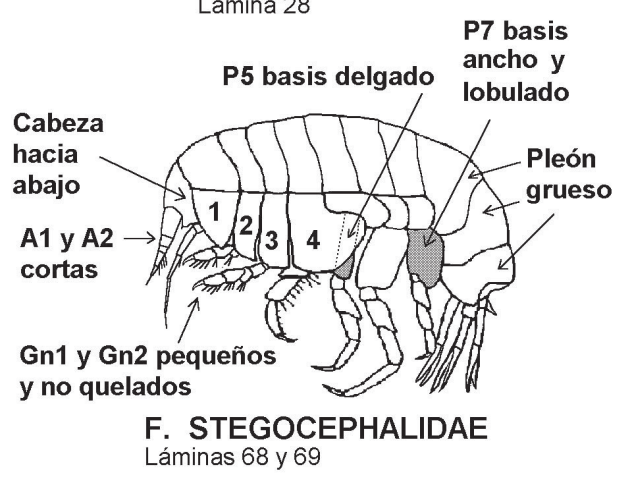

A1 con primer artejo

(Ver también Lepechinella de la F. Dexaminidae)
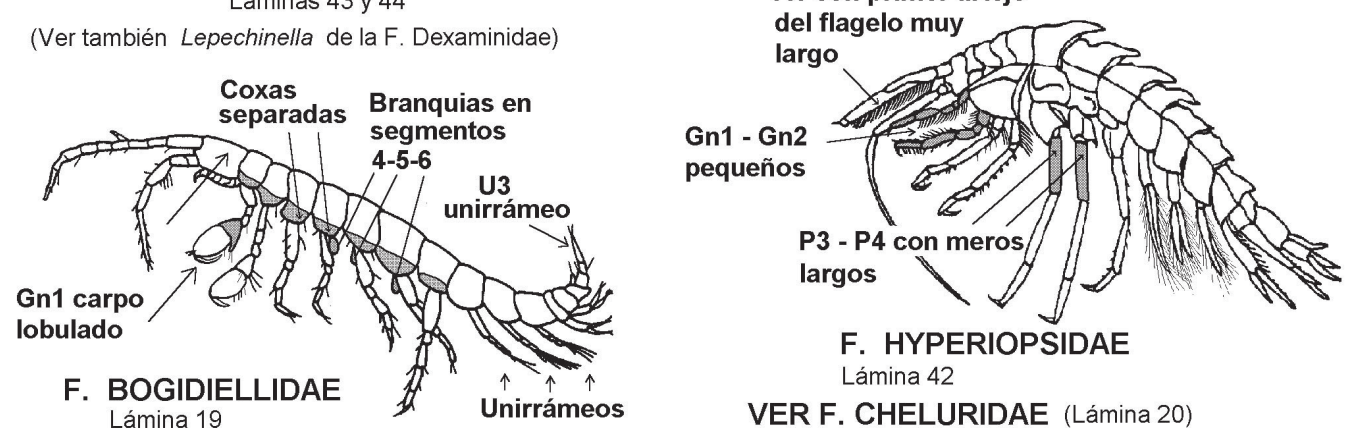

F. HYPERIOPSIDAE

Lámina 42

VER F. CHELURIDAE (Lámina 20)

Lámina 3.- Grupo de Familias con Géneros con coxas atípicas. 


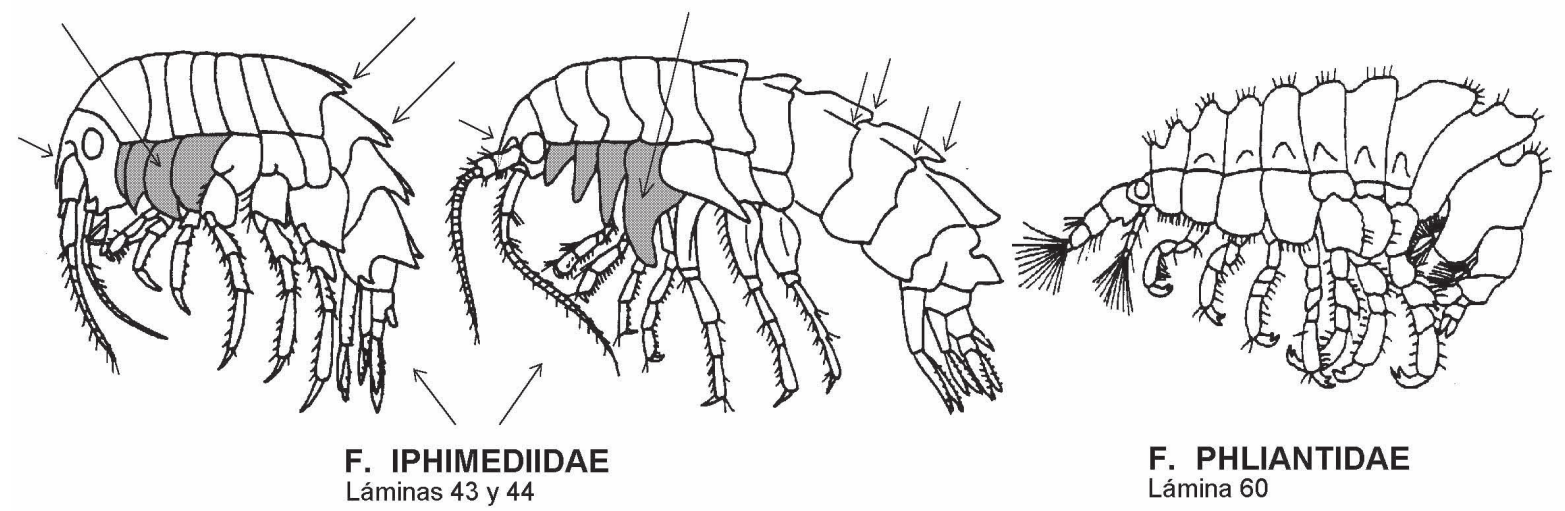

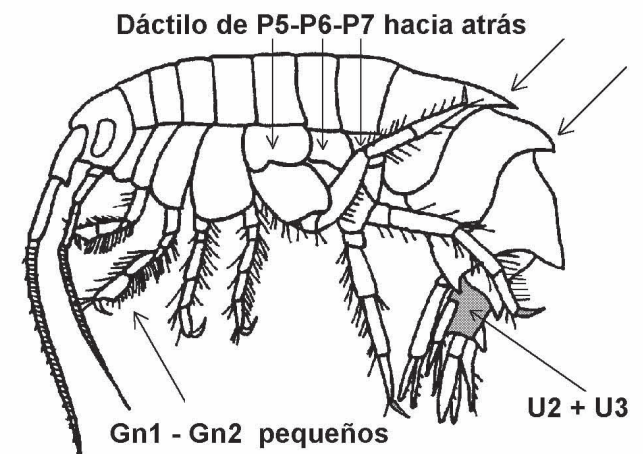

F. DEXAMINIDAE Láminas 29 y 30

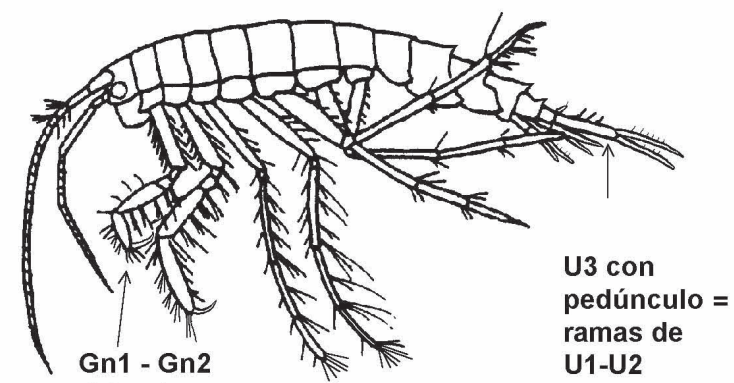

delgados y casi no quelados

\section{F. MELPHIDIPPIDAE} Lámina 53

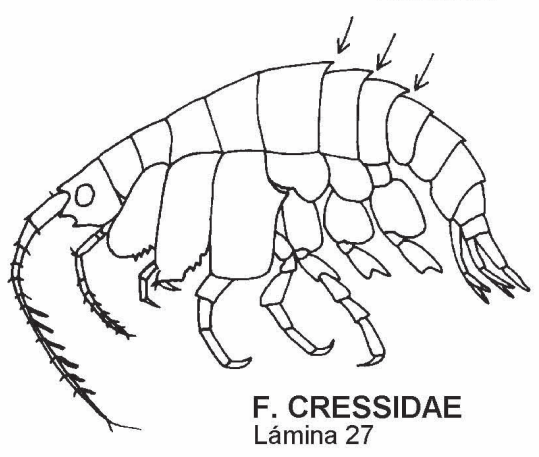

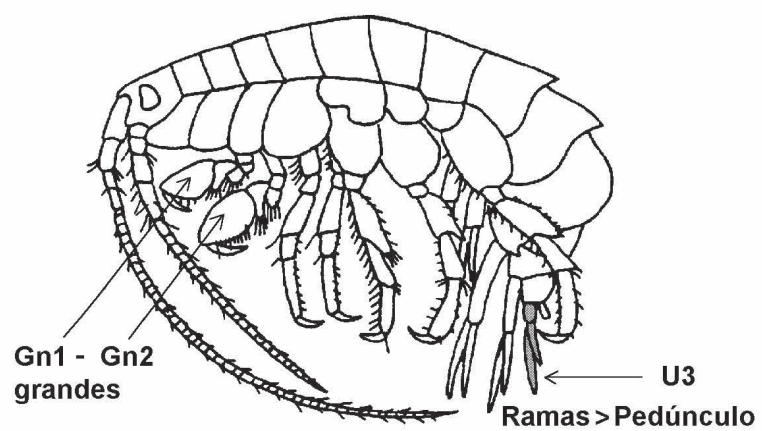

F. PLEUSTIDAE
Lảminas 63 y 64

R. int. > R. ext.

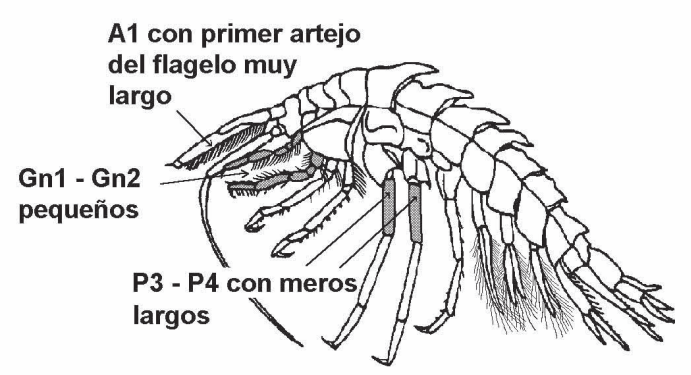

F. HYPERIOPSIDAE Lámina 42

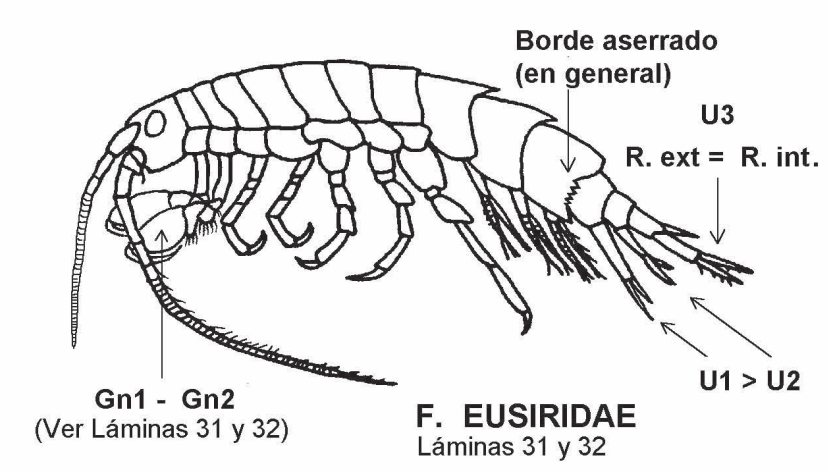

Lámina 4.- Grupo de Familias con Géneros que présentan el dorso dentado. 


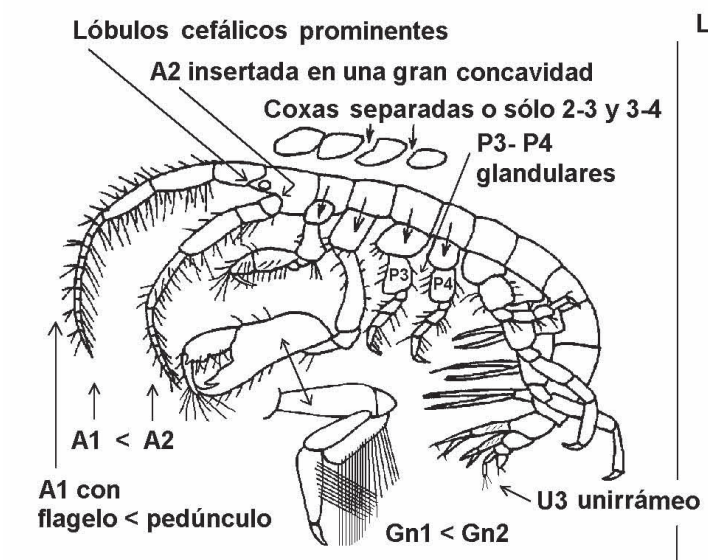

Lóbulos cefálicos no muy prominentes

A2 no insertada en una gran concavidad Coxas juntas o coxas 1-2 separadas
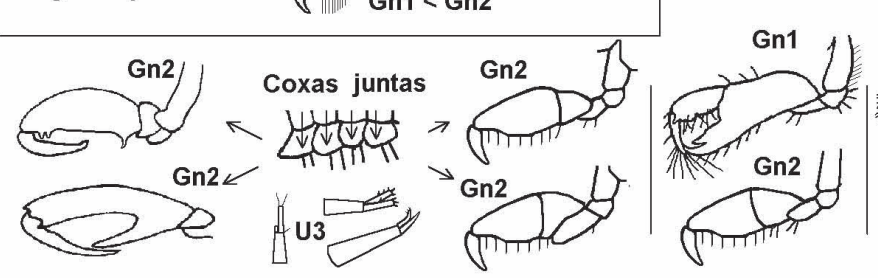

$\sigma^{\prime}$

P3-P4 glandulares

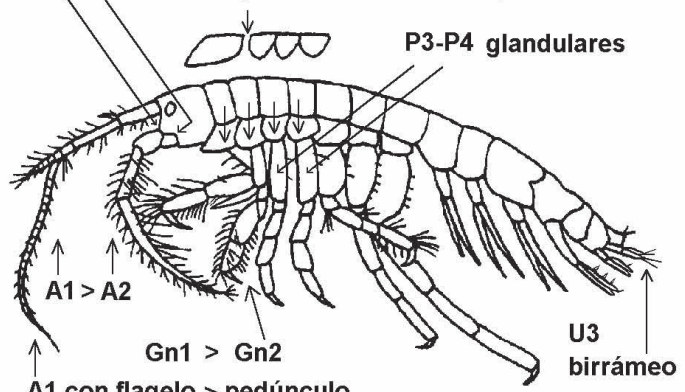

A1 con flagelo > pedúnculo

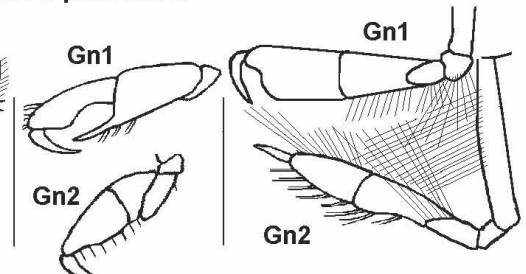

\section{F. COROPHIIDAE - ISCHYROCERIDAE}

Láminas 22 a 25
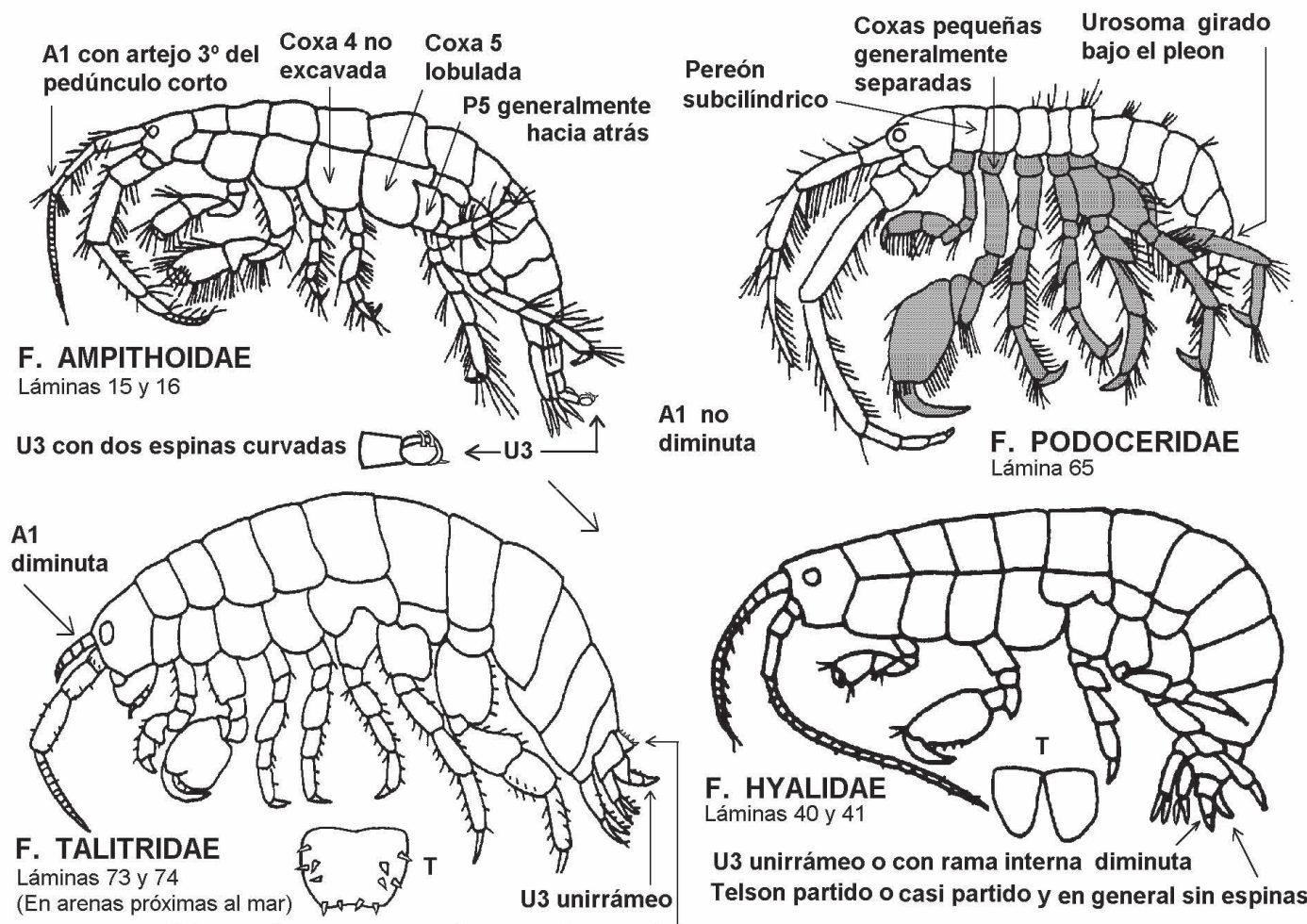

U3 unirrámeo o con rama interna diminuta

Telson partido o casi partido y en general sin espinas Telson entero y en general con muchas espinas

VER F.STENOTHOIDAE (Lámina 70), F. CHELURIDAE (Lámina 20) y F. PHLIANTIDAE (Lámina 60)

Lámina 5.- Grupo de Familias con Géneros que presentan el telson grueso y blando. 


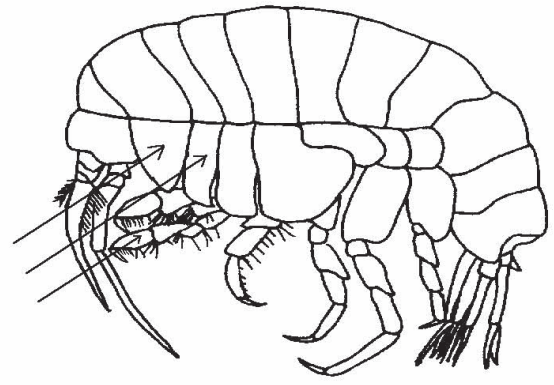

F. STEGOCEPHALIDAE Láminas 68 y 69
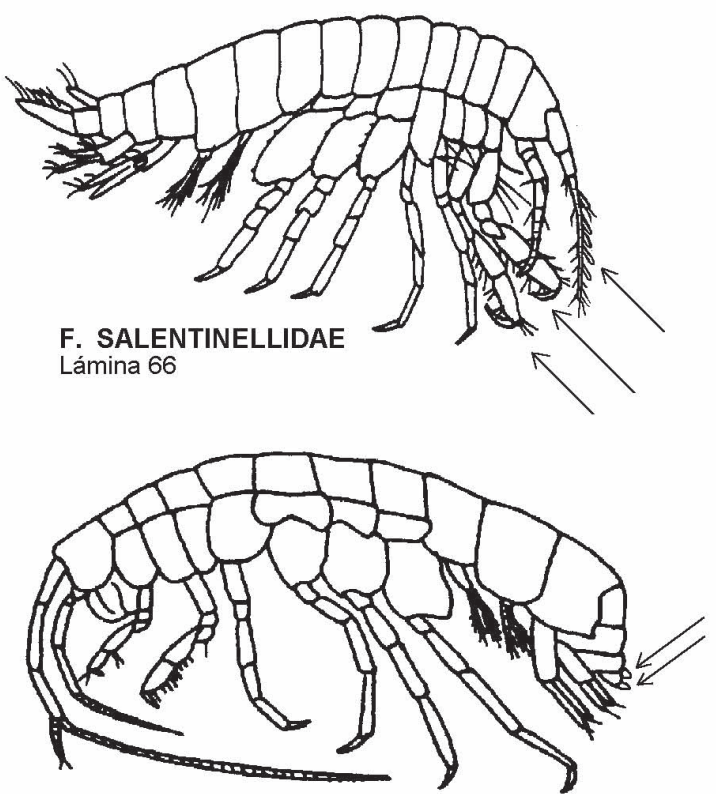

F. METACRANGONICTIDAE

Lámina 54

(Agua dulce)

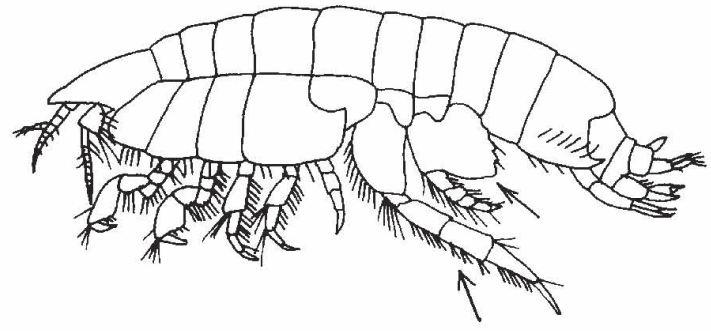

F. PHOXOCEPHALIDAE

Láminas 61 y 62

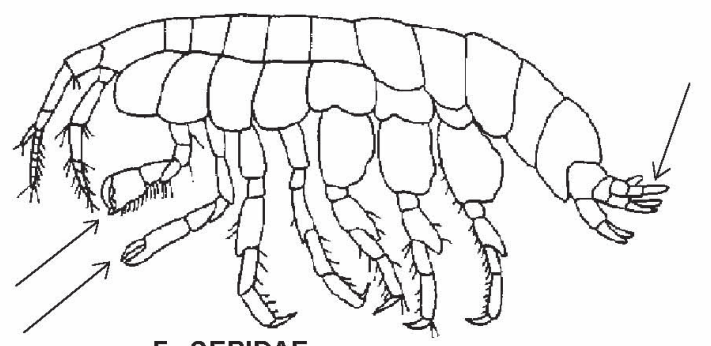

F. SEBIDAE

Lámina 67
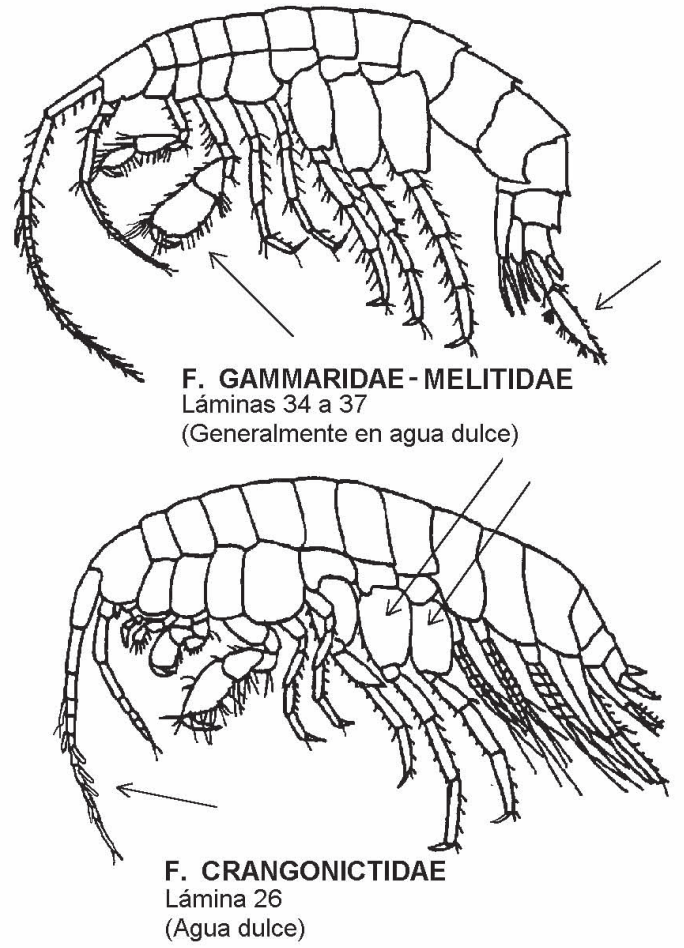

(Agua dulce)

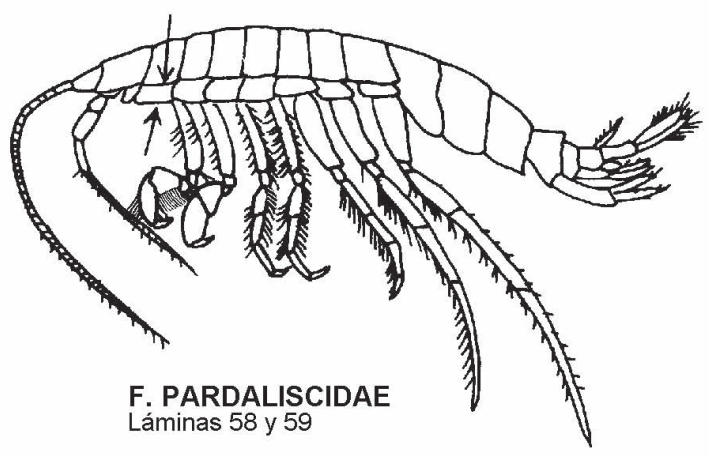

VER TAMBIÉN: F. AMPELISCIDAE (Lámina 11), F. BOGIDIELLIDAE (Lámina, 19),

F. HAUSTORIIDAE (Lámina 38), F. HYPERIOPSIDAE (Lámina 42),

F. LILGEBORGIIDAE (Lámina 46) y G. Sensonator (Lámina 76)

Lámina 6.- Grupo de Familias con Géneros que carecen de ojos. 

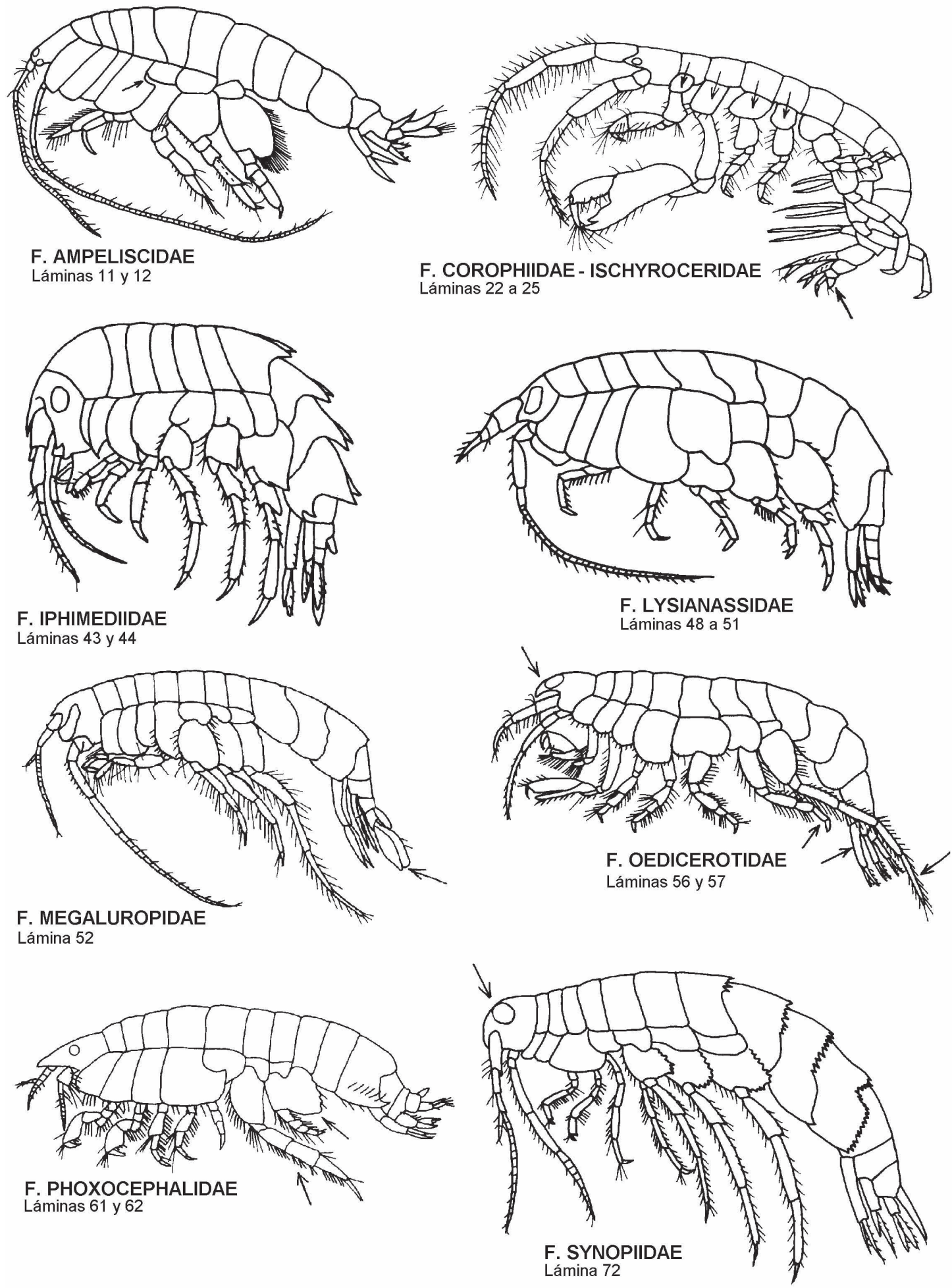

VER TAMBIÉN: F. GAMMARIDAE - MELITIDAE (Lámina 34) y F. HAUSTORIIDAE (Lámina 38)

Lámina 7.- Grupo de Familias con Géneros con cabezas u ojos atípicos. 

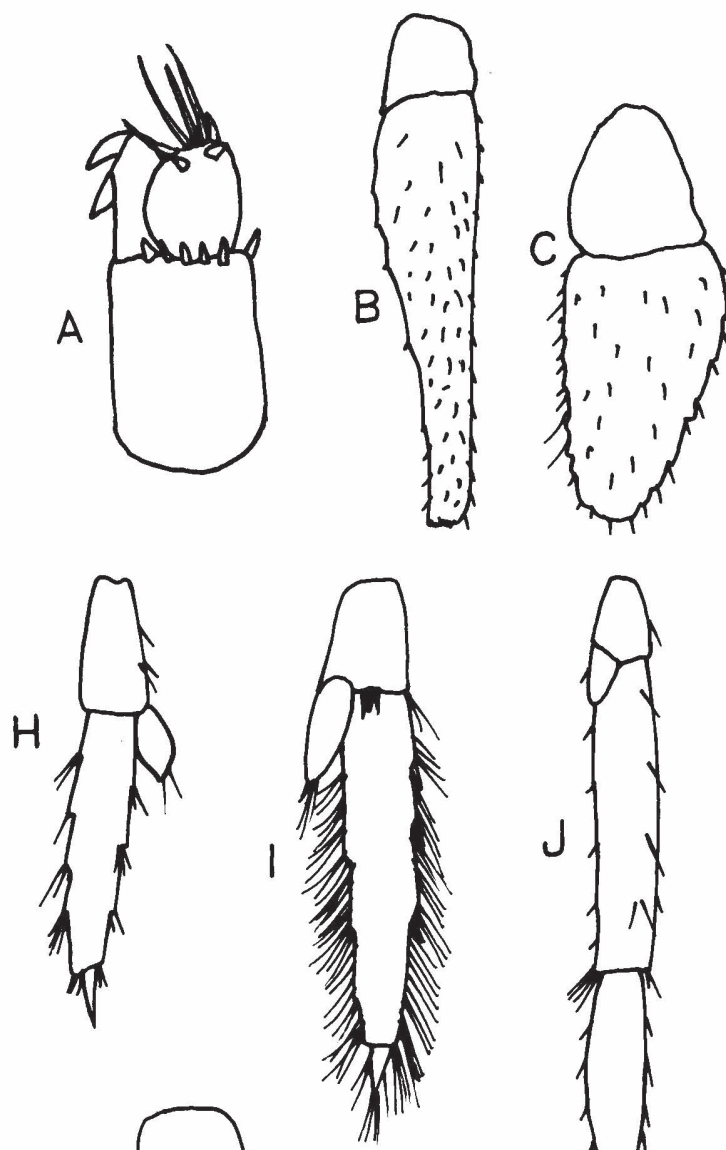

N
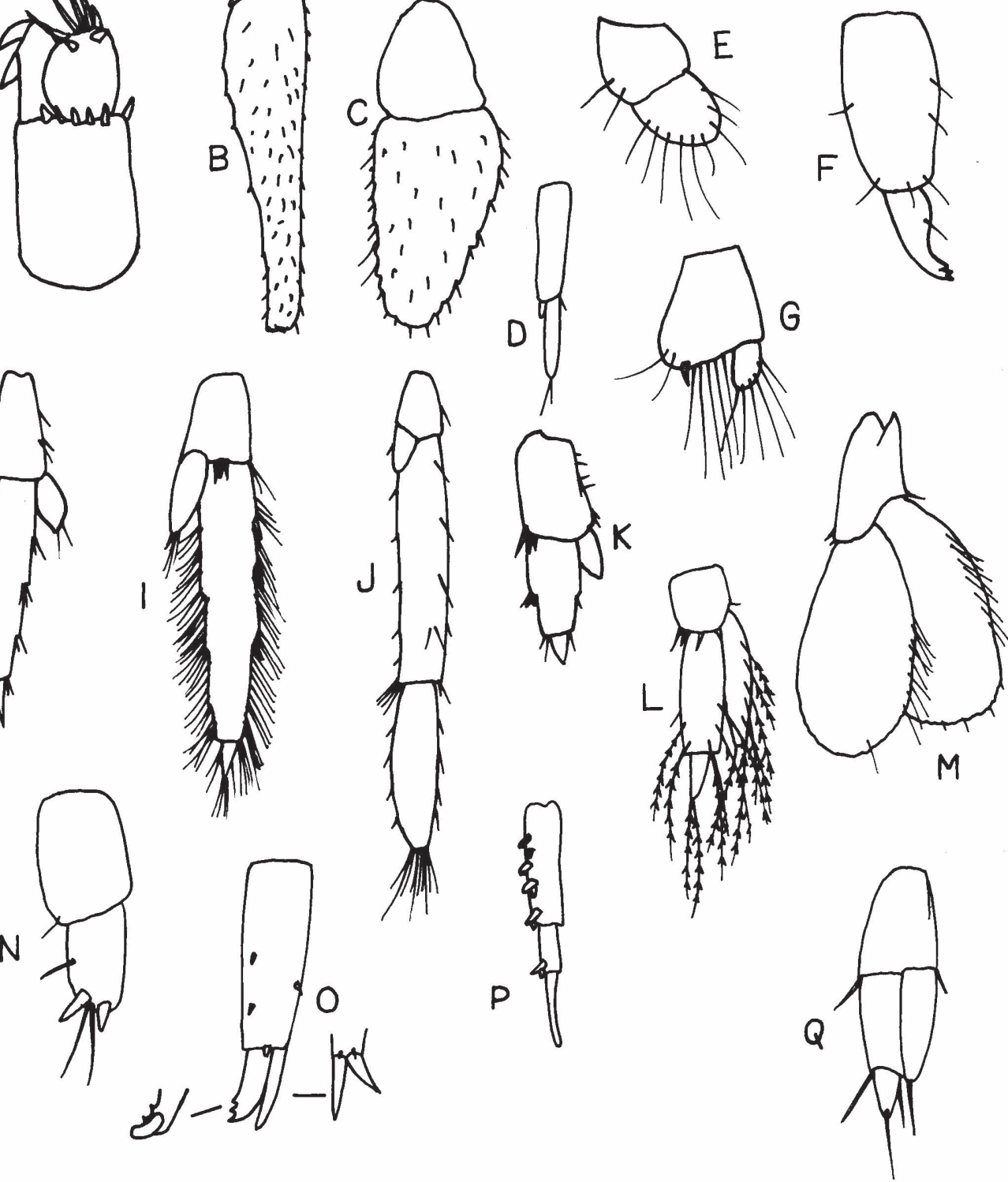

Lámina 8.- Grupo de Familias con Géneros con Urópodos 3 atípicos. (A) Ampithoidae (Lámina 15); (B y C) Cheluridae (Lámina 20); (D, E, F, G, N y O) Corophiidae - Ischyroceridae (Lámina 22); (H, I, J y K) Gammaridae-Melitidae (Lámina 34); (L) Phoxocephalidae (Lámina 61); (M) Megaluropidae (Lámina 52); (P) Stenothoidae (Lámina 70) y (Q) Urothoidae (Lámina 75). 


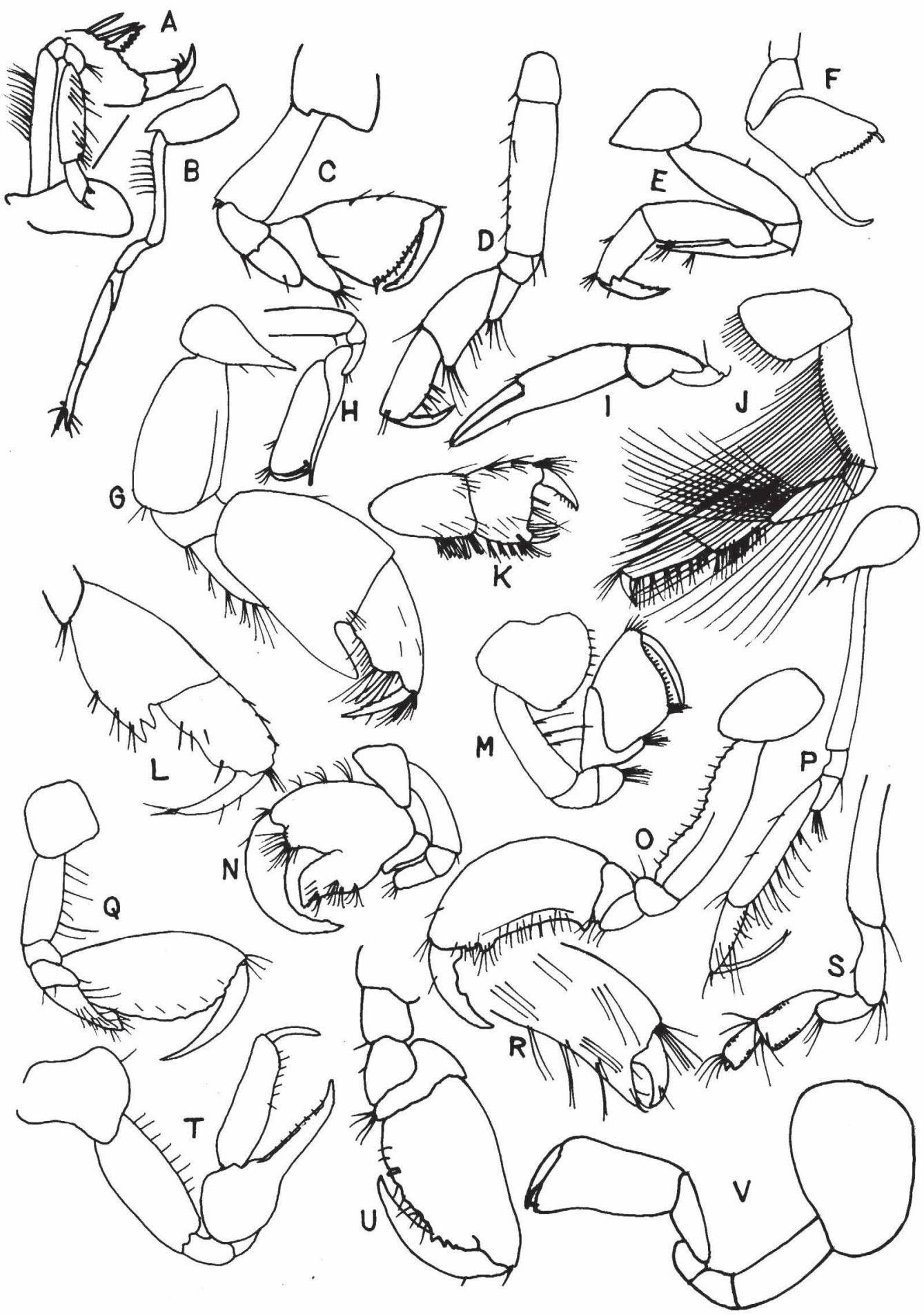

Lámina 9. Grupo de Familias con Géneros con Gnatópodos atípicos. (A) Synopiidae (Lámina 72); (B) Iphimediidae (Lámina 43); (C) Amphilochidae (Lámina 13); (D, E, G, J, K, L, N, O y P) Corophiidae-Ischyroceridae (Lámina 22); (F, R y S) Lysianassidae (Lámina 48); (H) Oedicerotidae (Lámina 56); (I) Sebidae (Lámina 67); (M) Eusiridae (Lámina 31); (Q y T) Leucothoidae (Lámina 45); (U) Stenothoidae (Lámina 70) y (V) Phoxocephalidae (Lámina 61). 


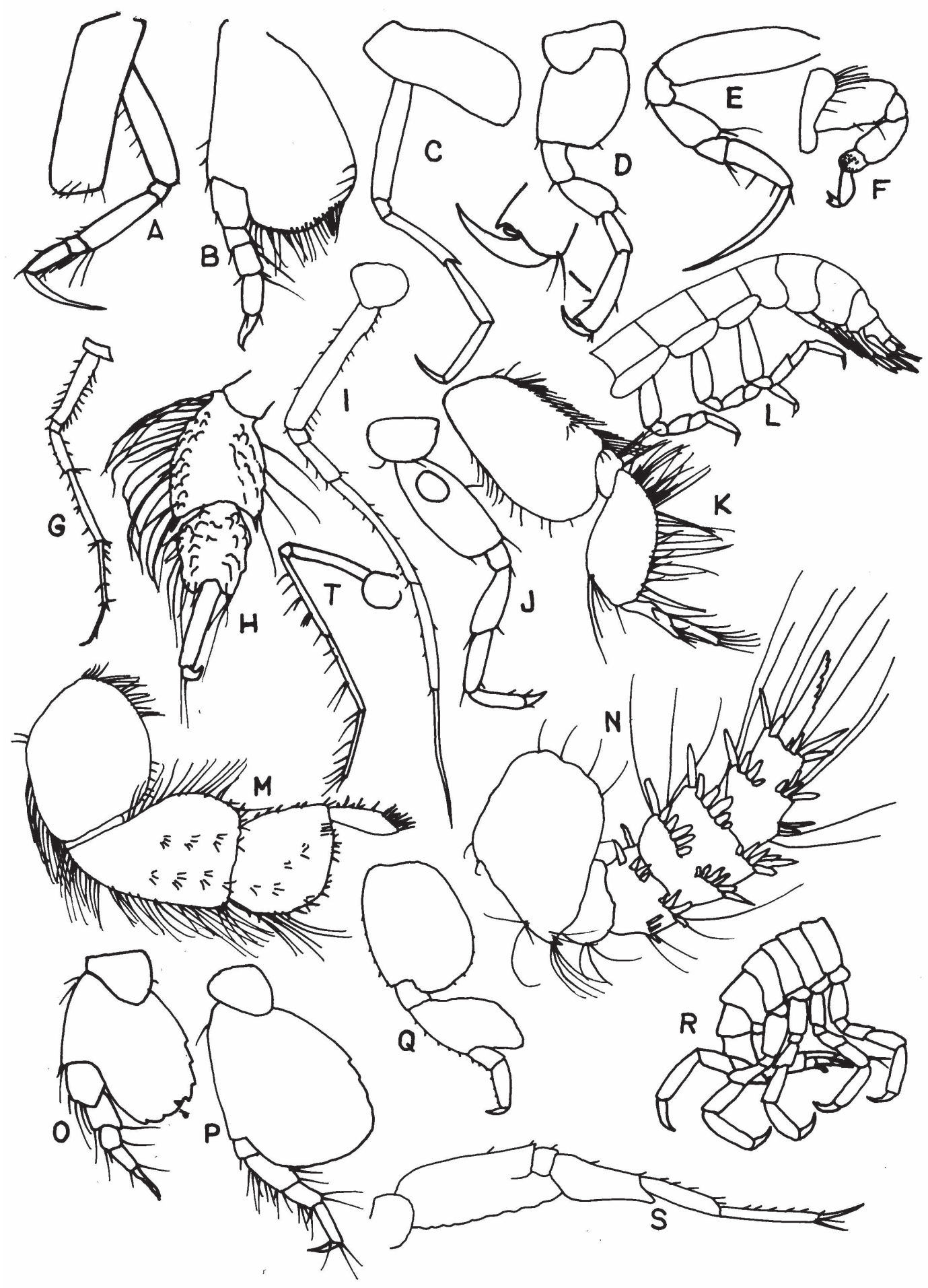

Lámina 10. Grupo de Familias con Géneros con Pereiópodos P3-P7 atípicos. (A y B) Ampeliscidae (Lámina 11); (C) Amphilochidae (Lámina 13); (D) Biancolinidae (Lámina 18); (E y F) Corophiidae-Ischyroceridae (Lámina 22); (G y H) Dexaminidae (Lámina 29); (I) Eusiridae (Lámina 31); (J) Bogidiellidae (Lámina 19); (K y M) Haustoriidae (Lámina 38); (L) Colomastigidae (Lámina 21); (N) Urothoidae (Lámina 75); (O y P) Phoxocephalidae (Lámina 61); (Q) Sebidae (Lámina 67); (R) Podoceridae (Lámina 65); (S) Synopiidae (Lámina 72) y (T) Melphidipiidae (Lámina 53). 

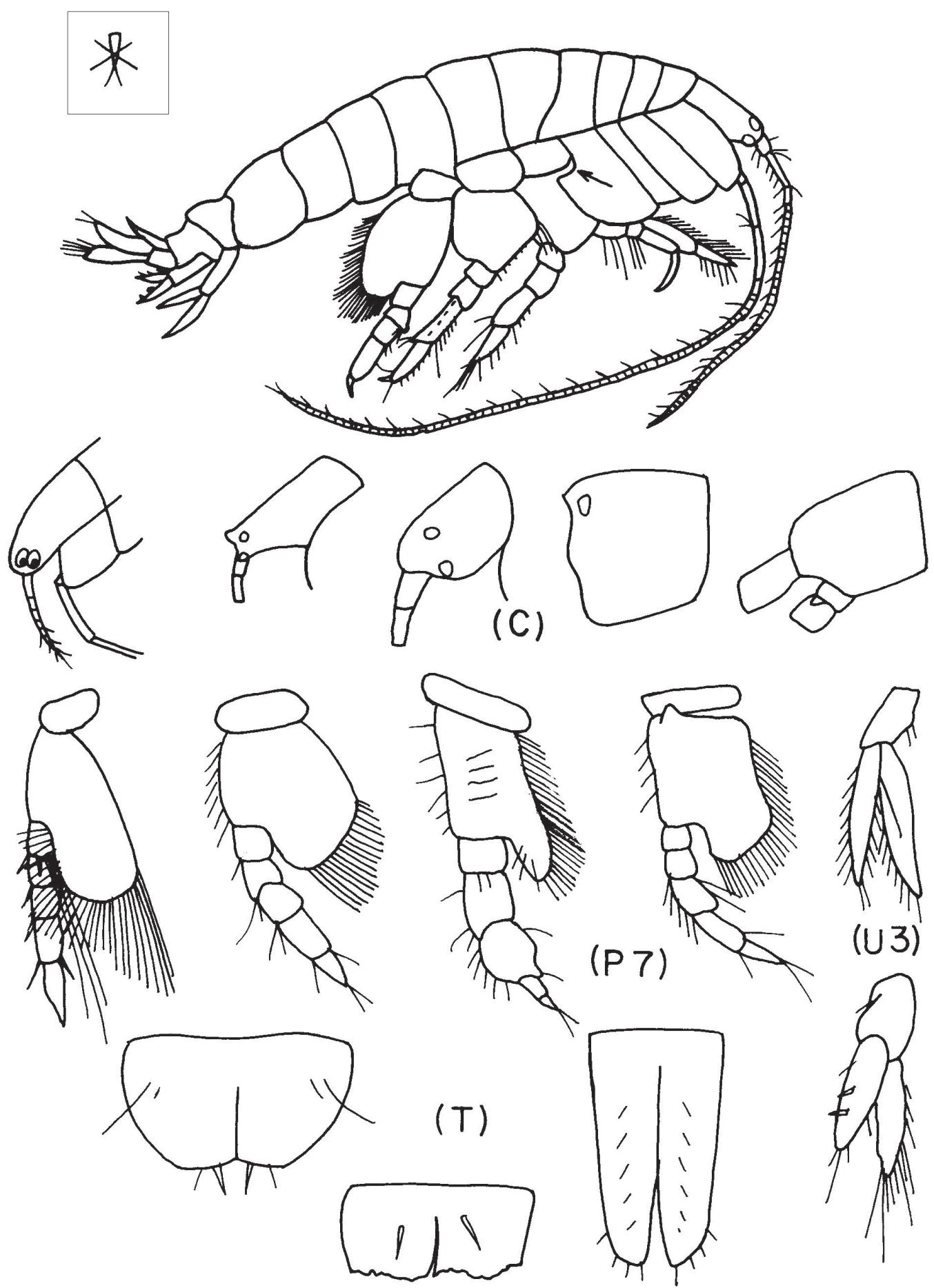

( $P 7)$
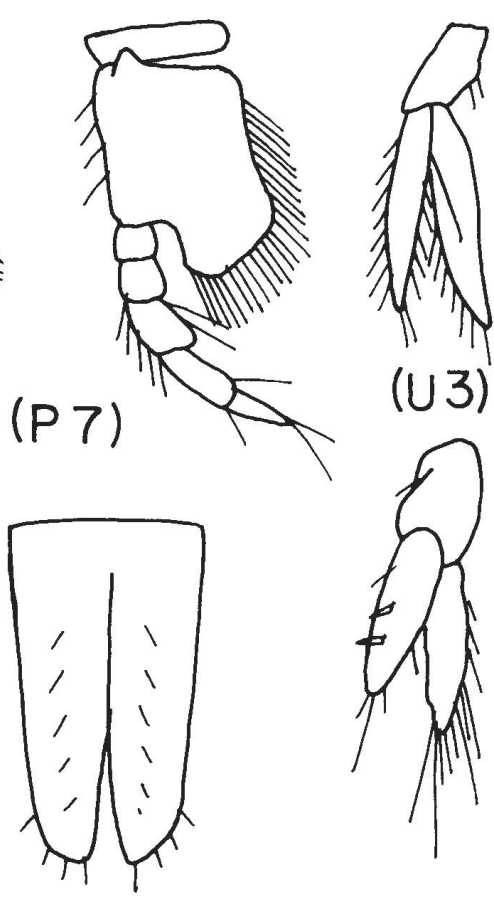

Lámina 11.— Familia AMPELISCIDAE (continúa). 


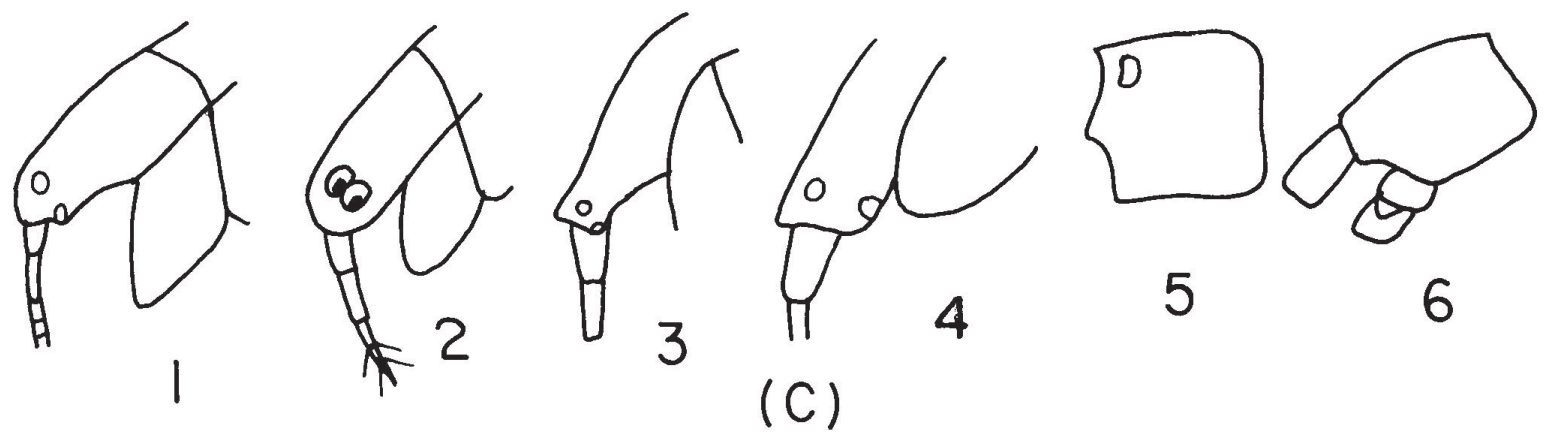

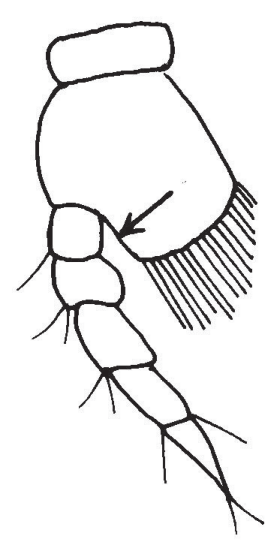

7

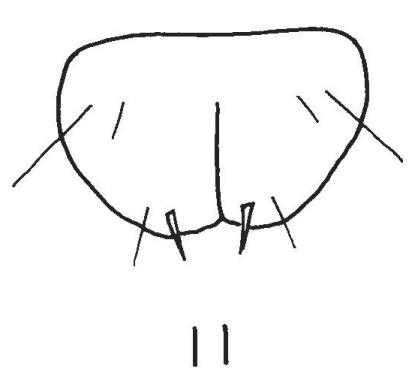

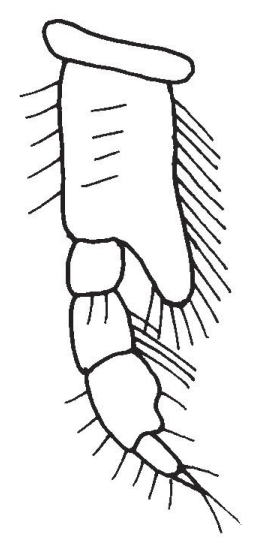

8

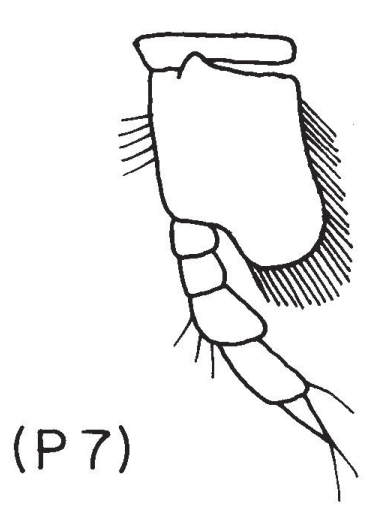

9

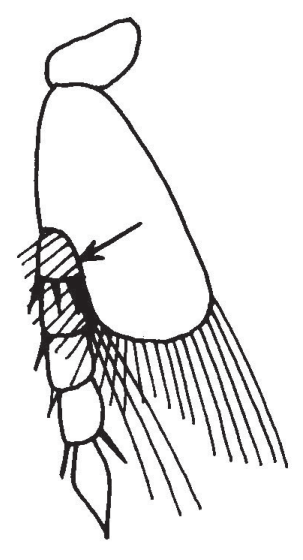

10
$(\mathrm{T})$

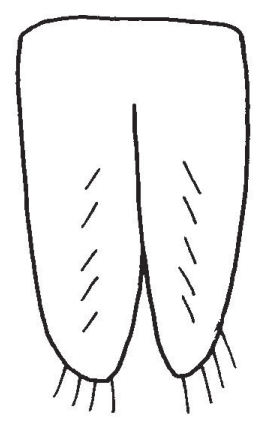

12

Lámina 12.- Familia AMPELISCIDAE (final).

1-3, 7, 12 Ampelisca;

4, 10, $13 \rightarrow$ Byblis;

4-6, 8-9, $11 \Rightarrow$ Haploops. 

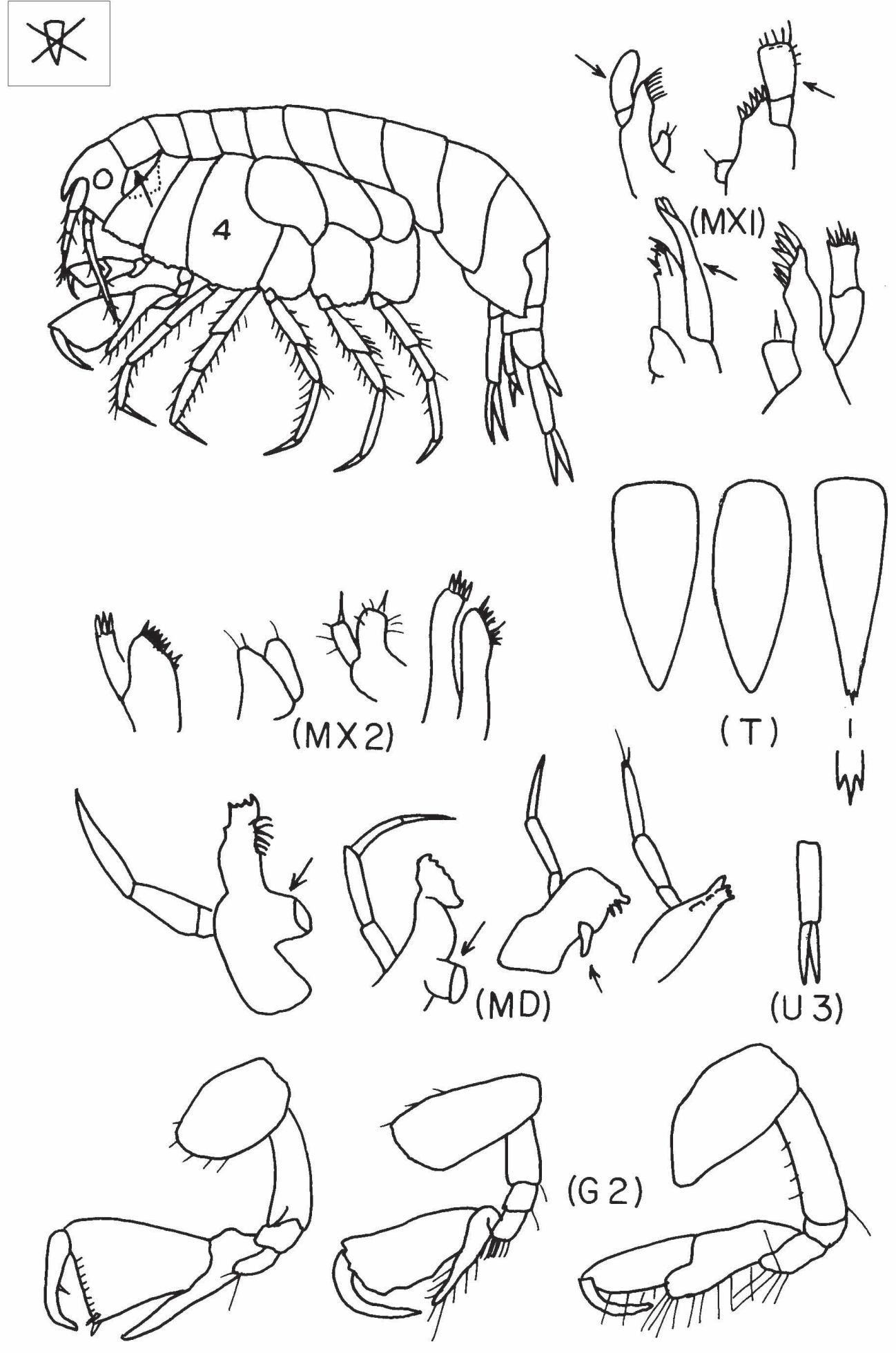

Lámina 13.- Familia AMPHILOCHIDAE (continúa). 

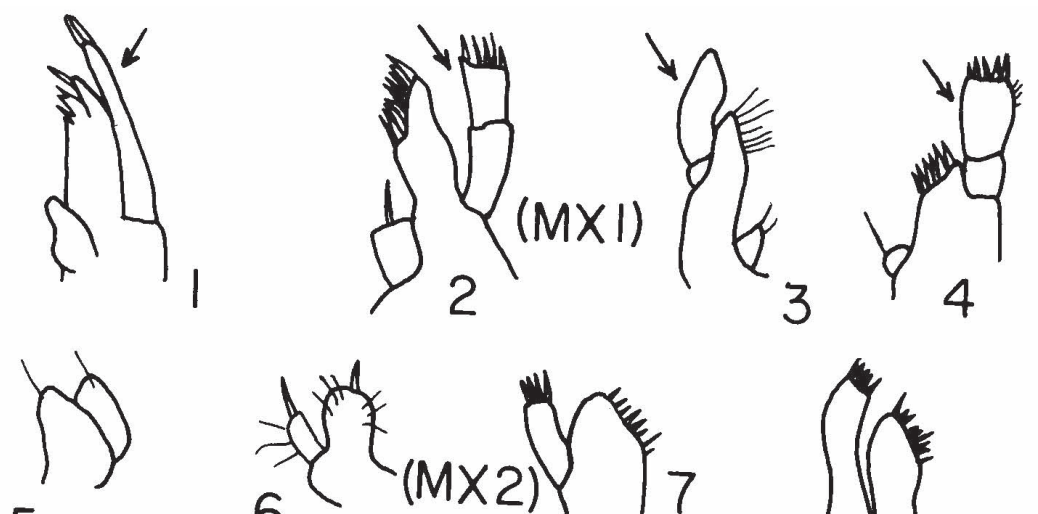

5
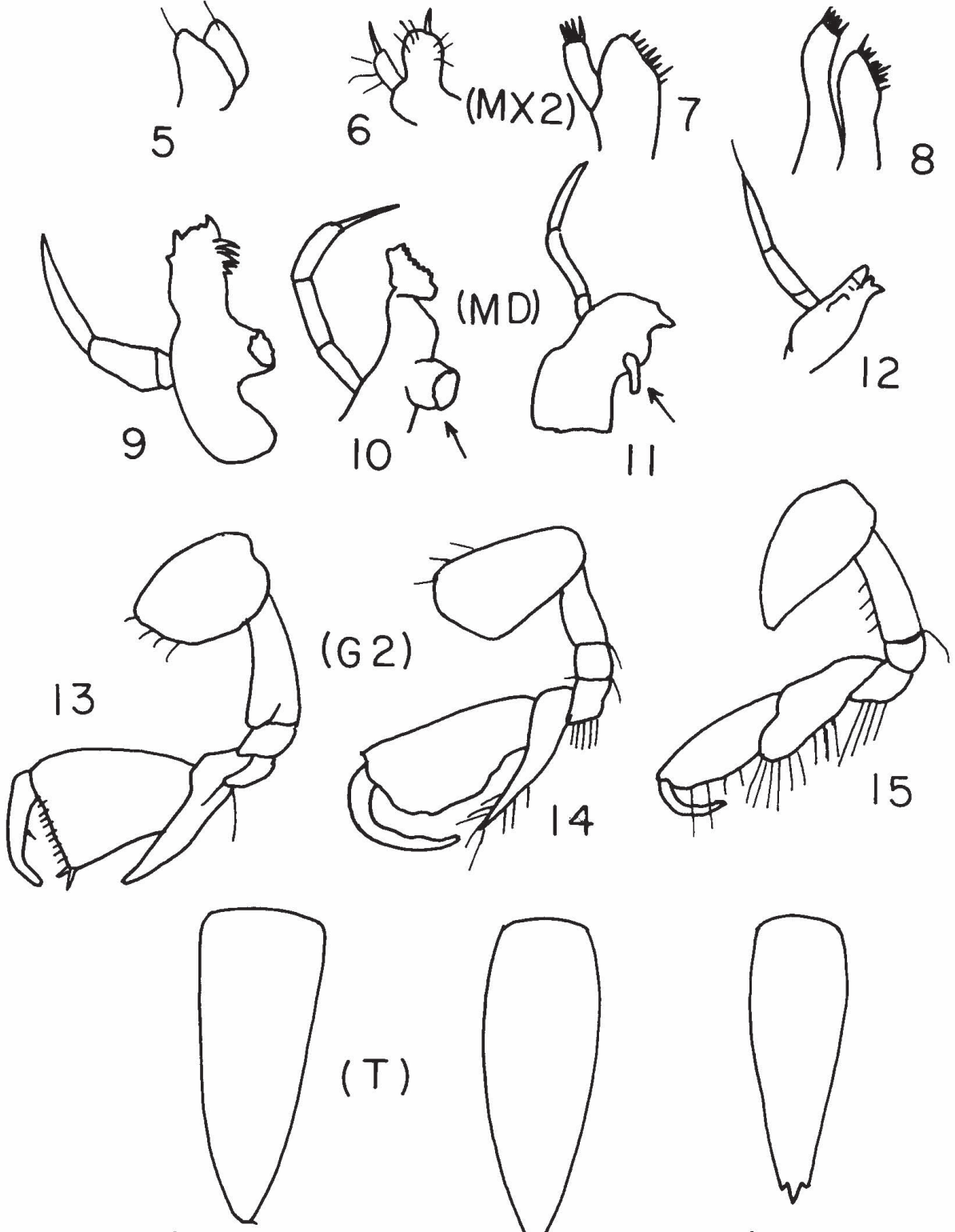

16
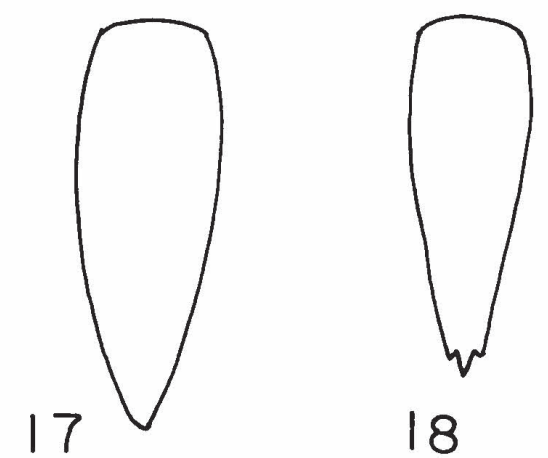

Lámina 14. Familia AMPHILOCHIDAE (final).

4, 5-6, 12, 13-14, $18 \Rightarrow$ Amphilochoides;

2, 8, 9, 13, 16-18 $\rightarrow$ Amphilochus;

2, 7, 10, 15, $18 \Rightarrow$ Gitana;

3, 5-6, 11, 14, $18 \Rightarrow$ Paramphilochoides (ver CYPROIDEIDAE). 


\section{我又对}
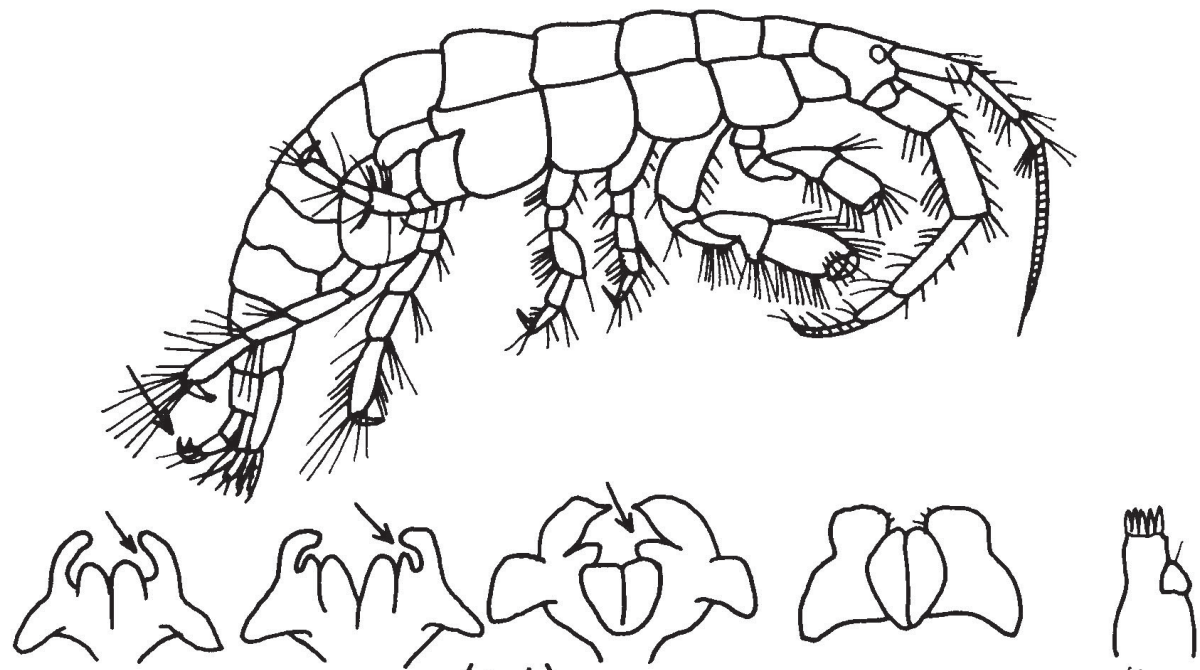

(LI)

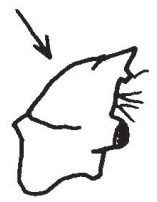

(MD) \#
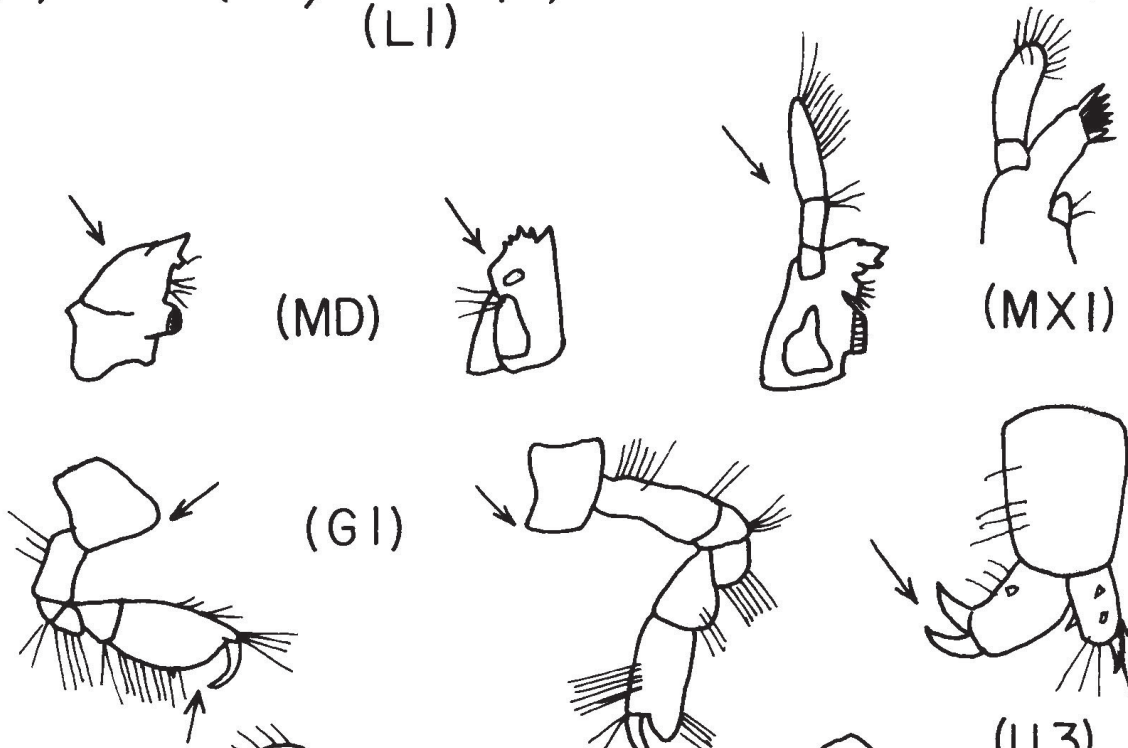

(GI)
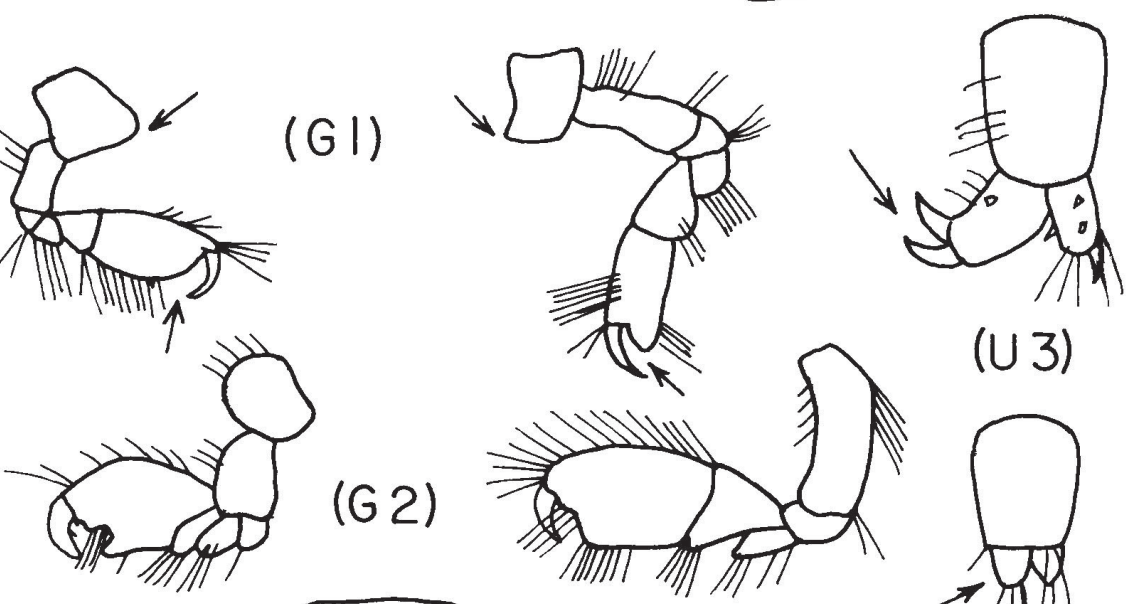

(G2)
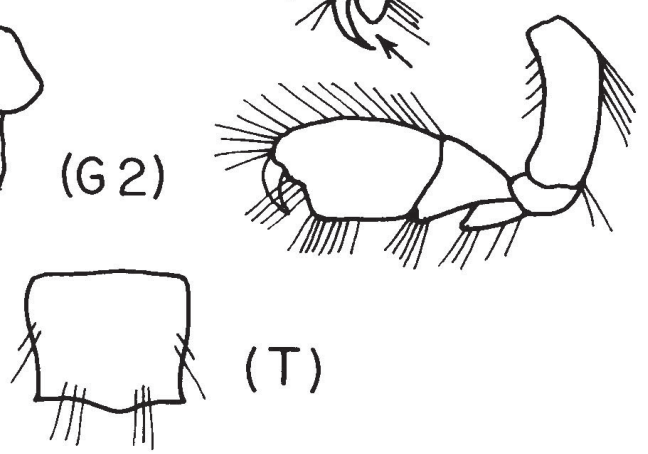

(U 3)

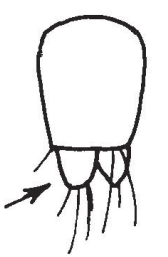

( $\mathrm{T})$

Lámina 15.— Familia AMPITHOIDAE (continúa). 

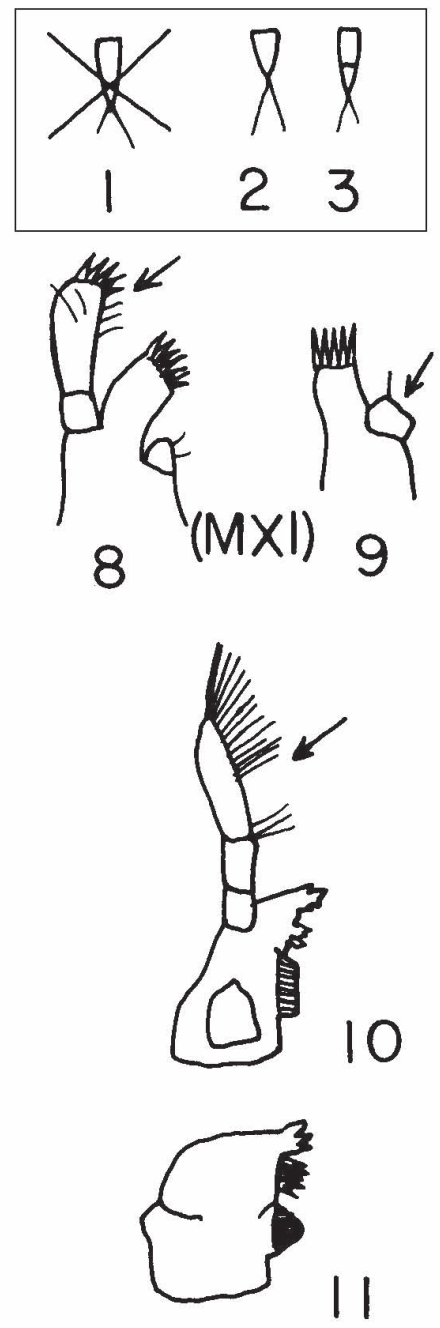

(MD)

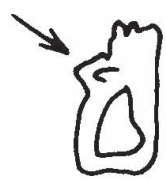

12
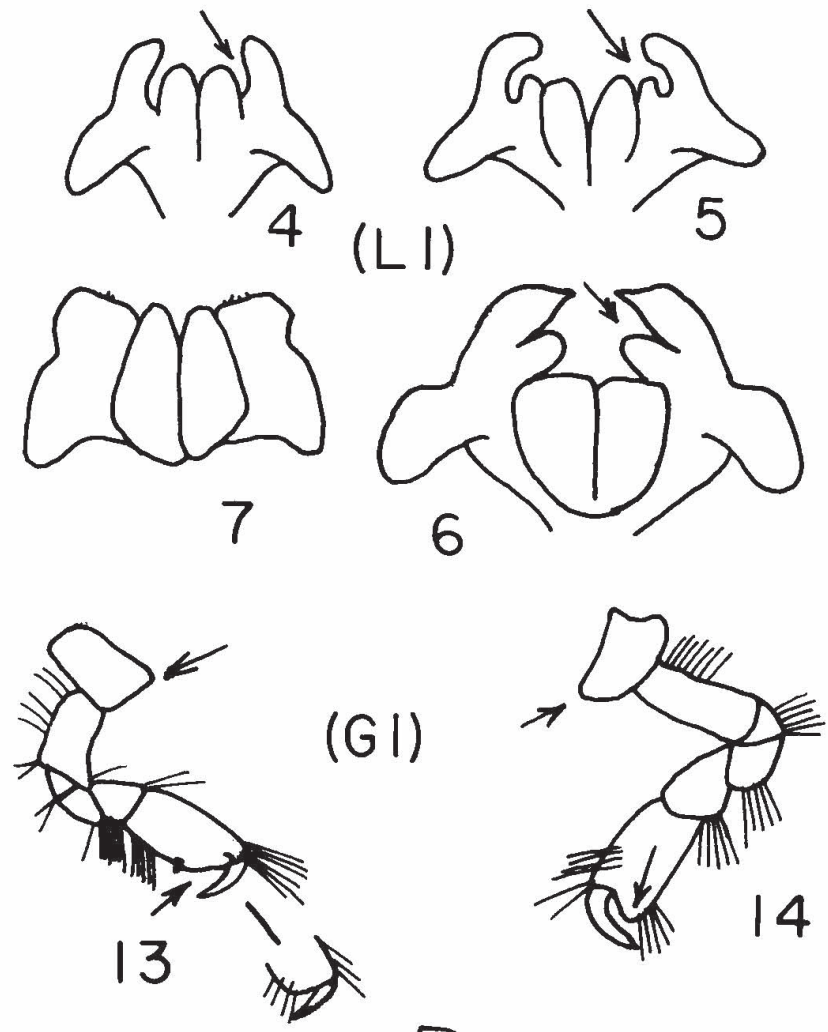

15
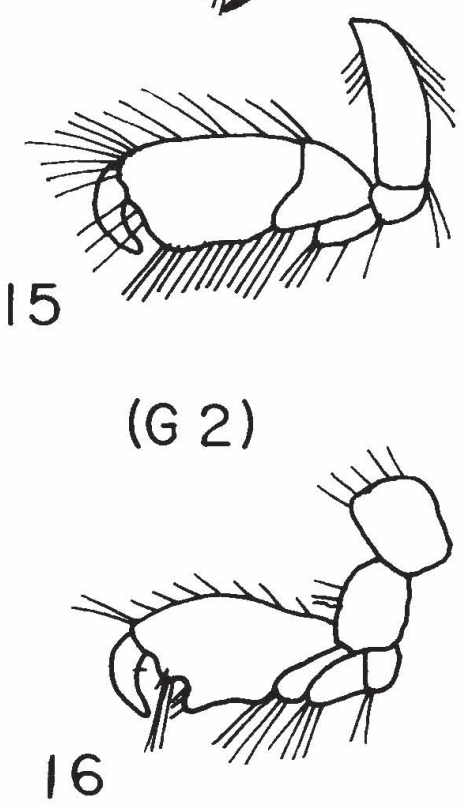

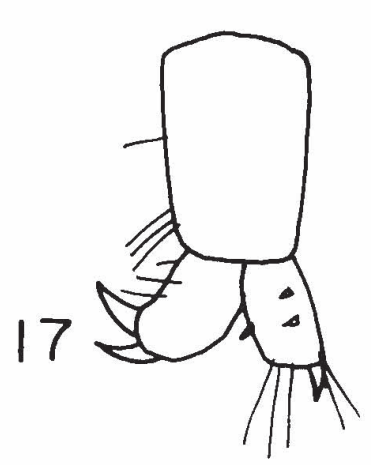

18

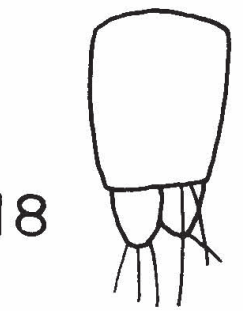

Lámina 16. - Familia AMPITHOIDAE (final).

1, 4-6, 9, 10, 12, 15, $17 \Rightarrow$ Ampithoe;

1, 7, 9, 13, 14, 16, 18 Amphitolina;

2-3, 4, 8, 10, 13, 15, $17 \Rightarrow$ Peramphithoe;

$1,4,8,11,13,15,17 \rightarrow$ Sunamphitoe. 

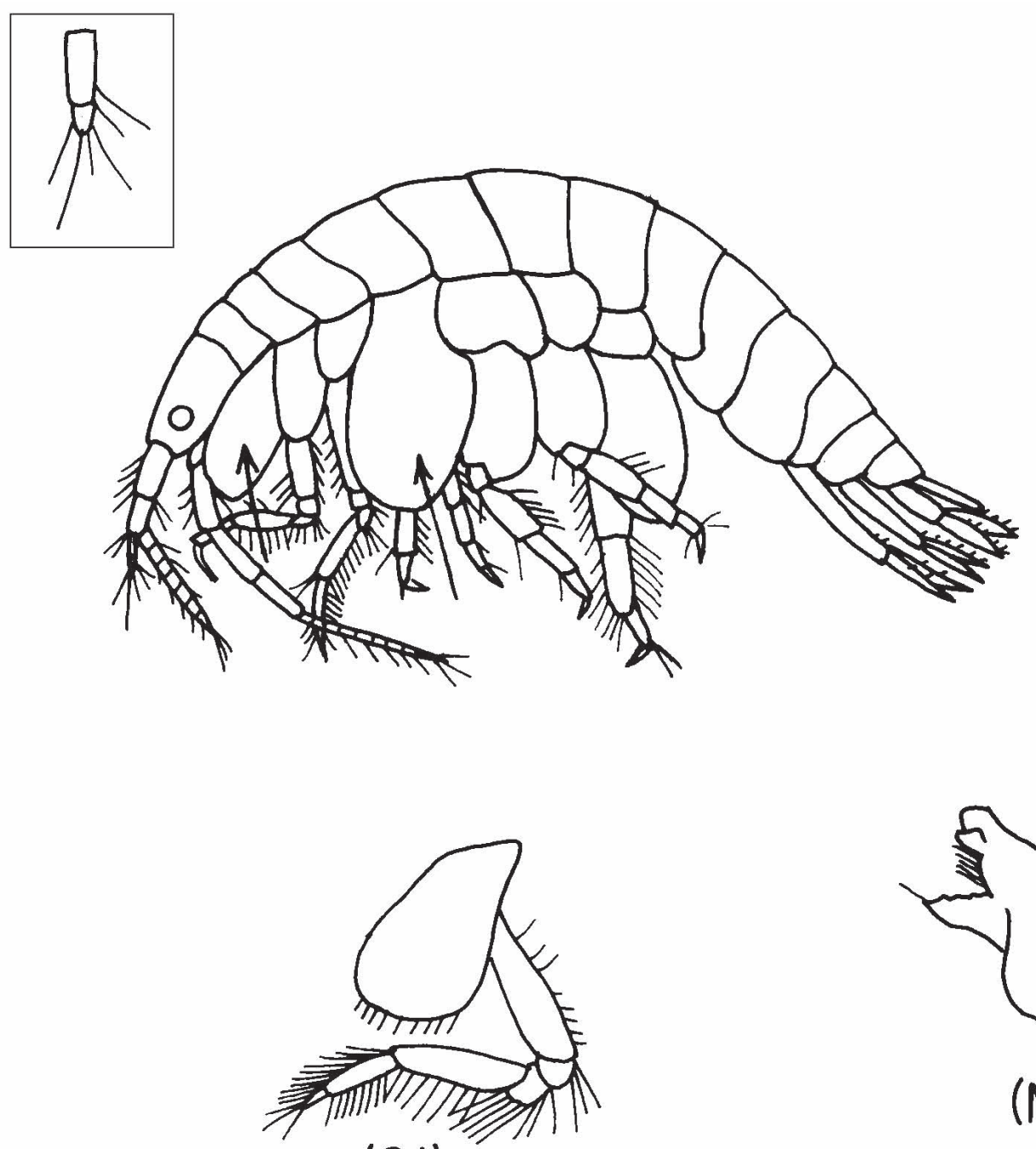

(GI)

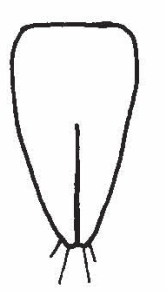

( $T$ )
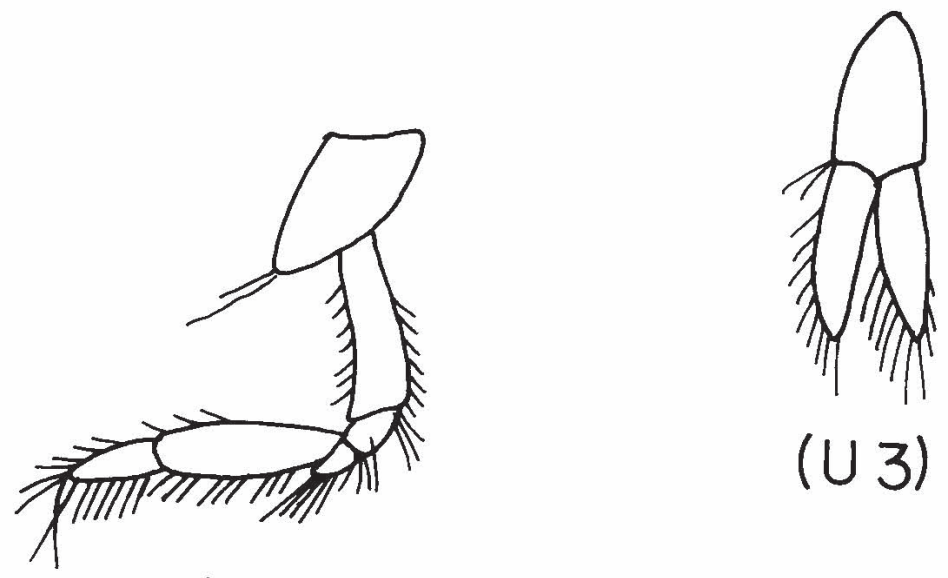

(U 3)

(G 2)

Lámina 17.— Familia ARGISSIDAE (monogenérica) $\Rightarrow$ Argissa. 

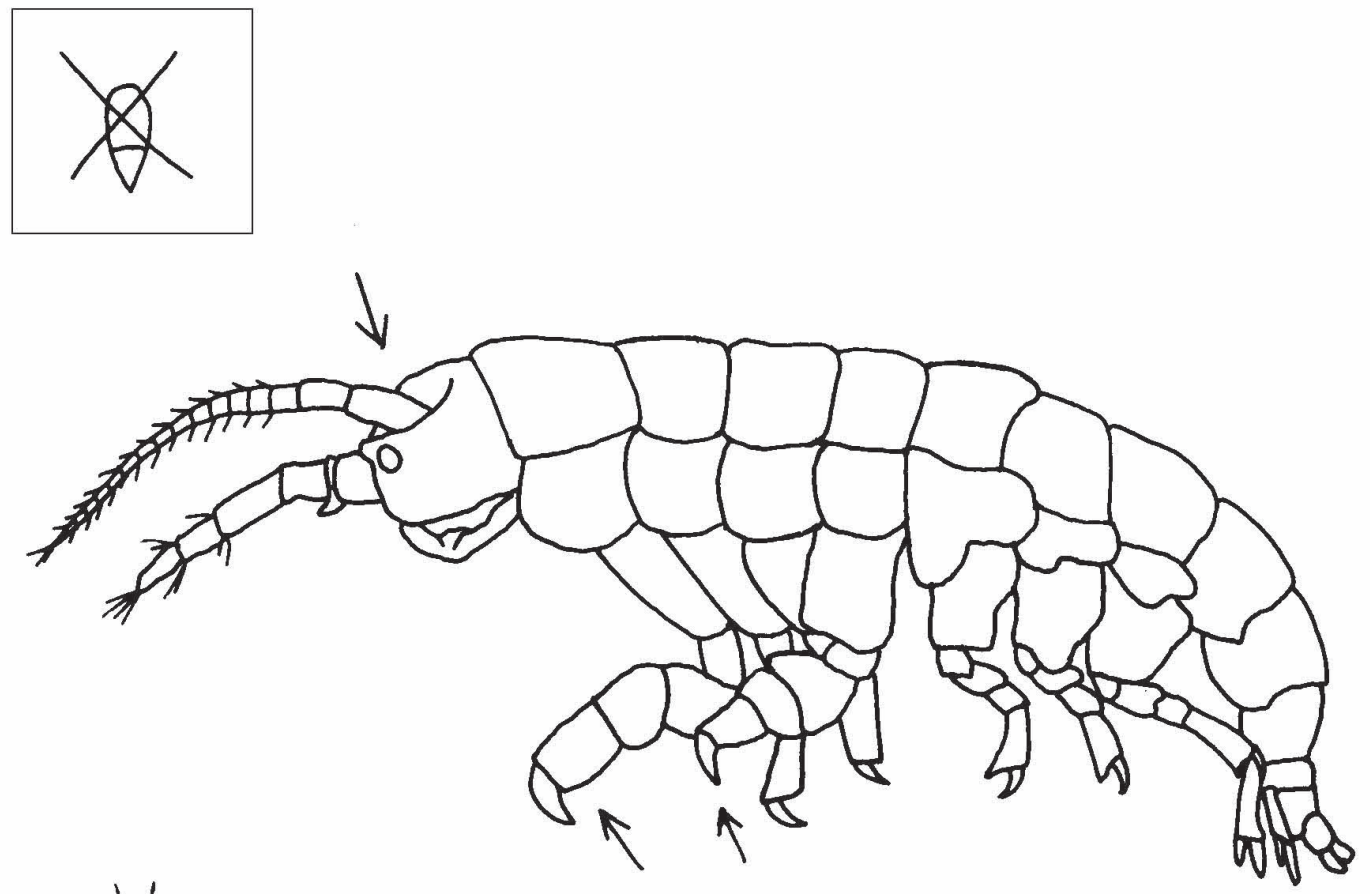

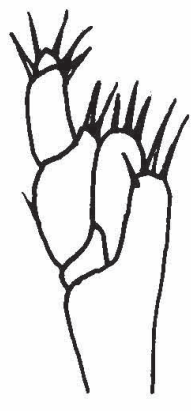

$(M \times P)$

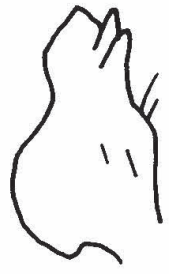

(MD)

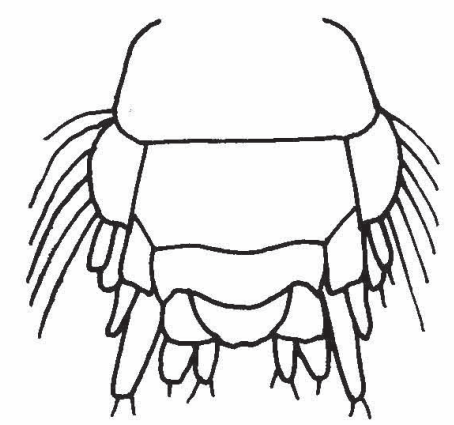

(UR)

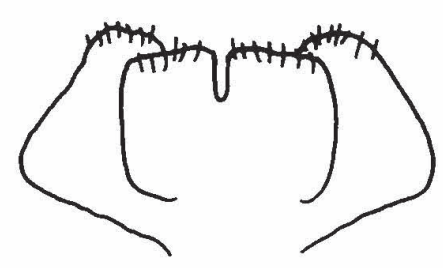

(LI)

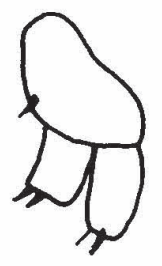

(U3)

Lámina 18.-Familia BIANCOLINIDAE (monogenérica) $\Rightarrow$ Biancolina. 


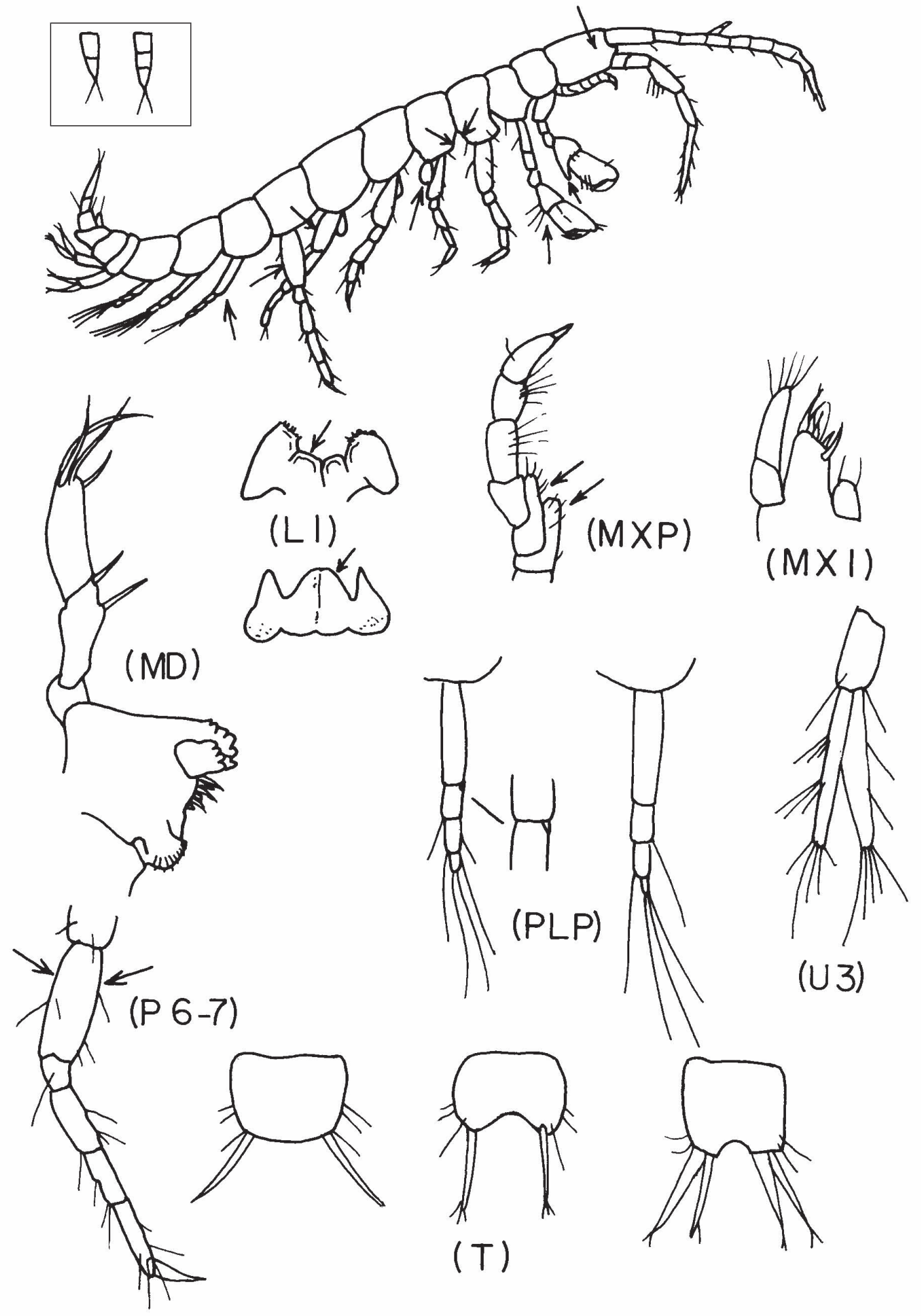

Lámina 19.- Familia BOGIDIELLIDAE (un género en el área) $\Rightarrow$ Bogidiella. 


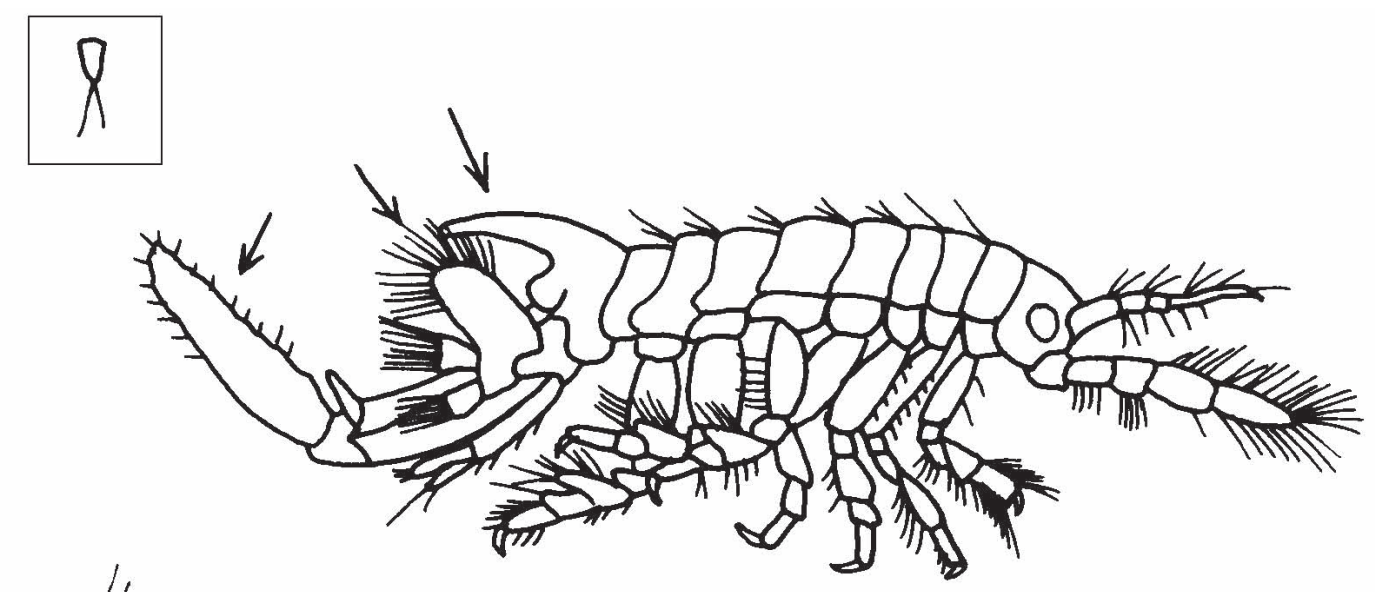

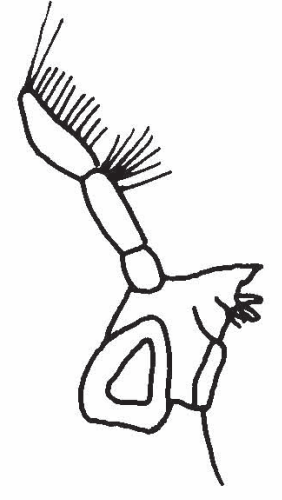

(MD)

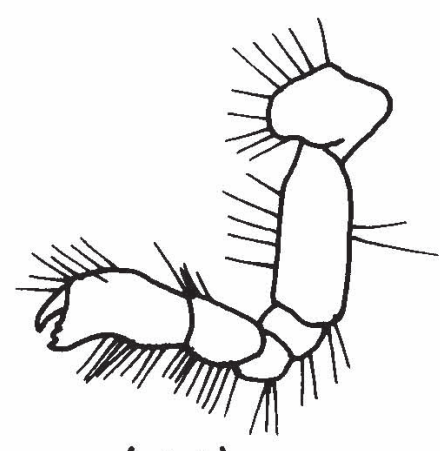

( $G \mid$ )
(G 2)

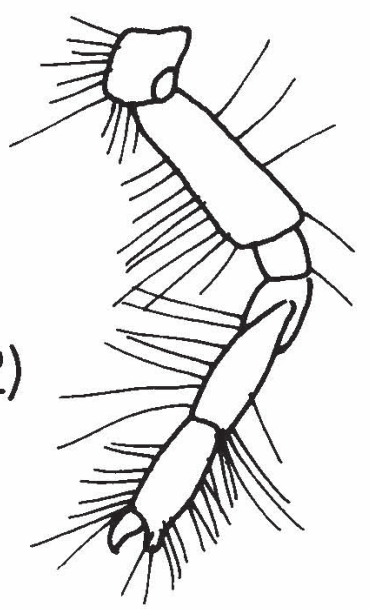

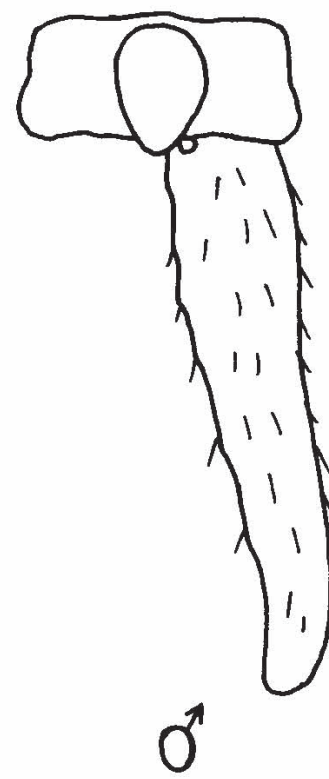

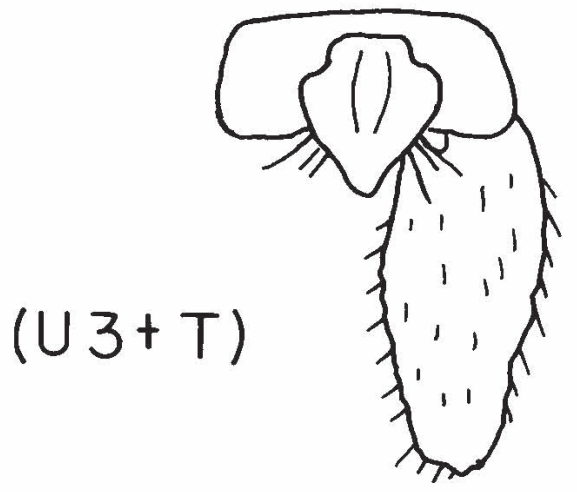

Q

Lámina 20.- Familia CHELURIDAE (un género en el área) $\Rightarrow$ Chelura. 


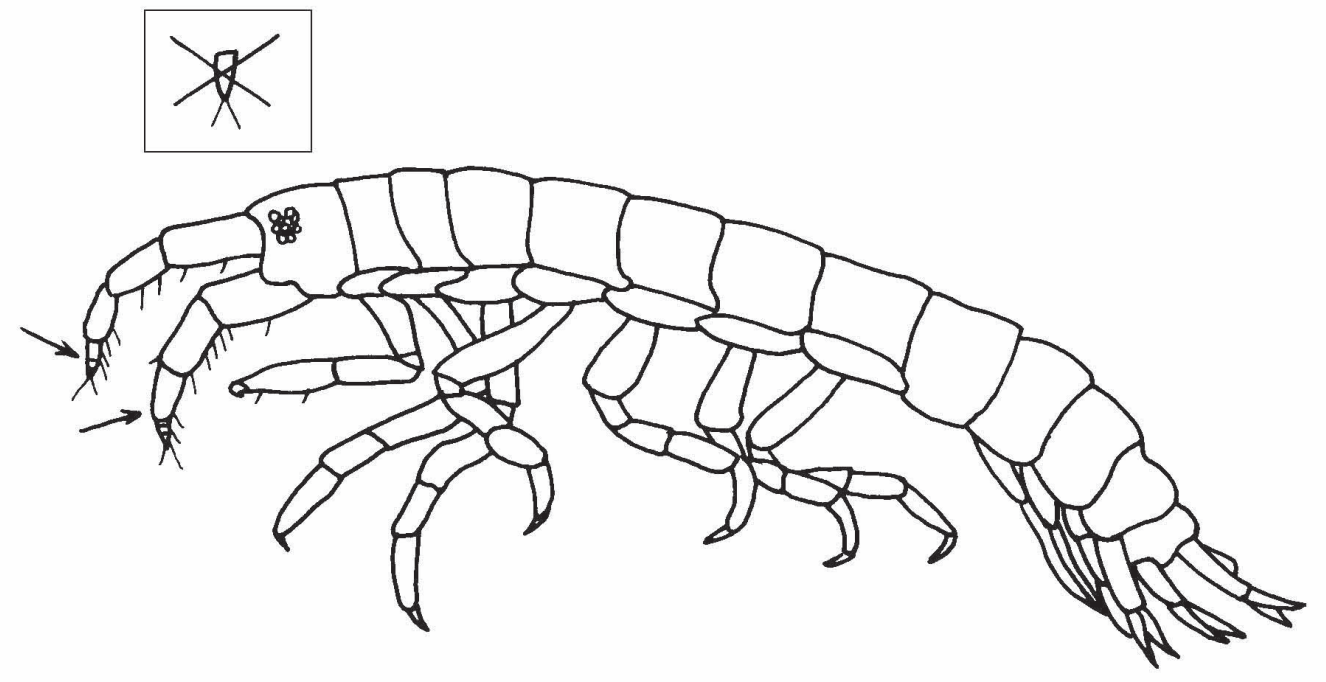

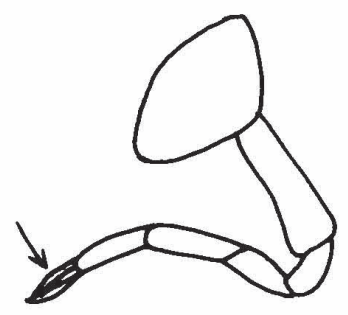

q

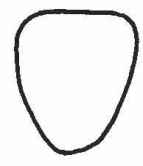

( $\mathrm{T}$ )

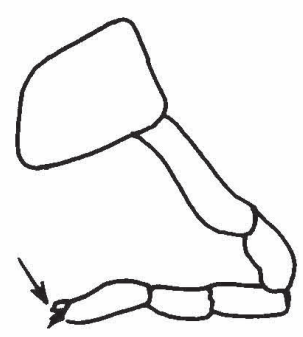

1

(GI)

(G 2)

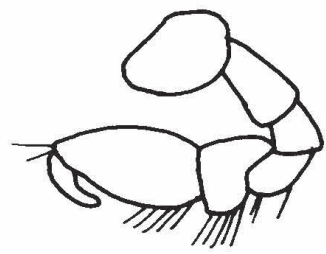

1
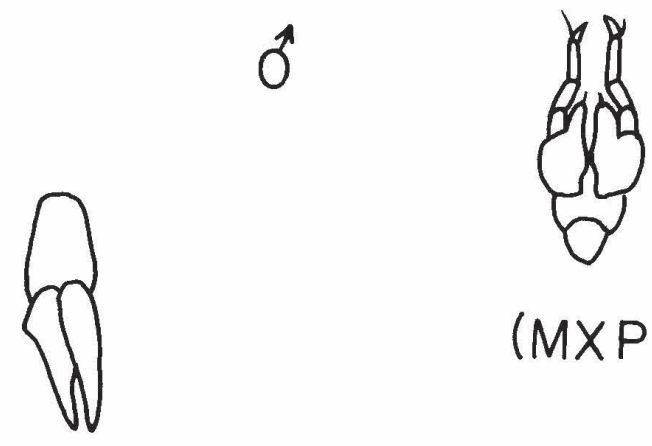

$(M \times P)$

Lámina 21.-Familia COLOMASTIGIDAE (un género en el área) $\Rightarrow$ Colomastix. 


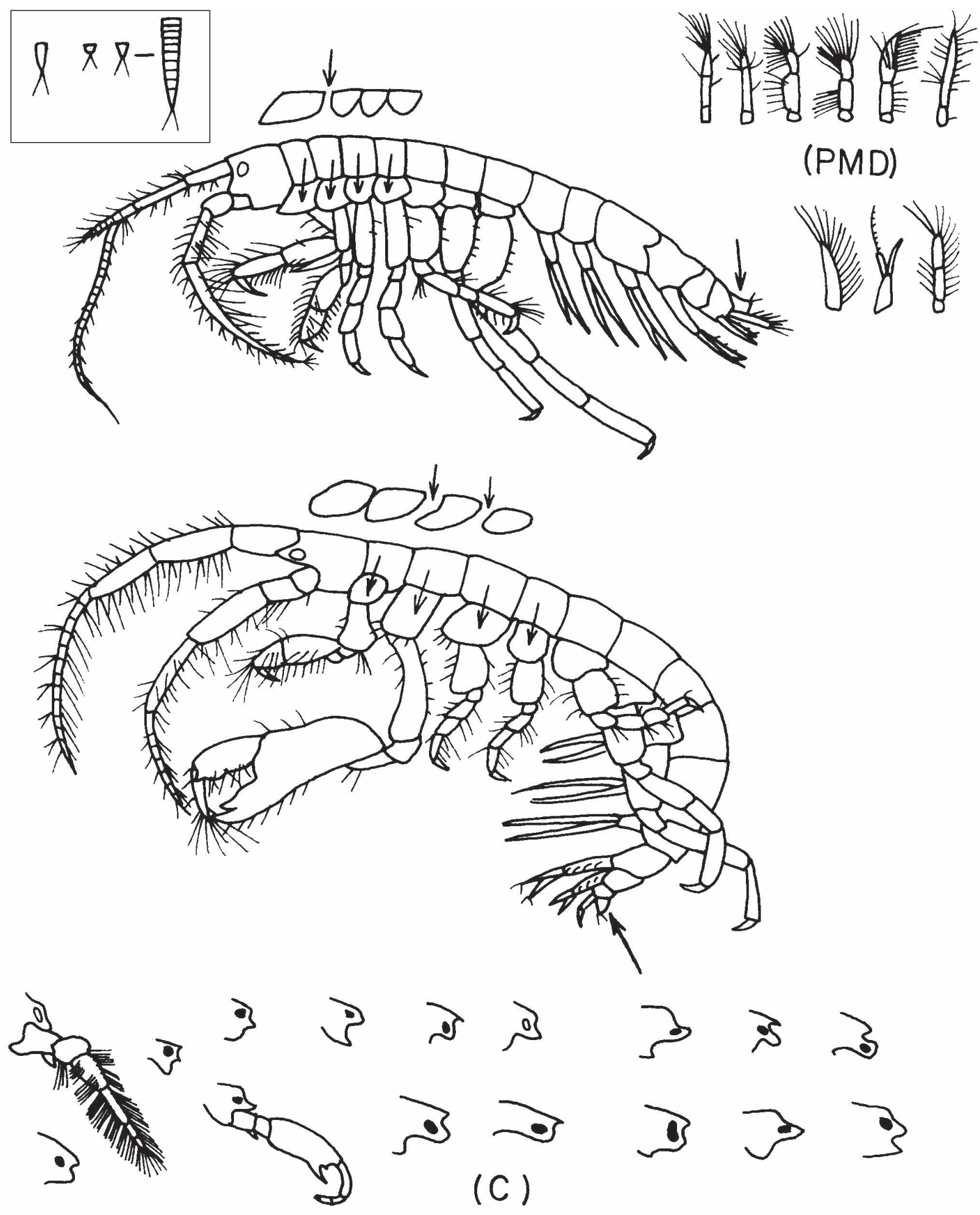

Lámina 22.— Familia COROPHIIDAE-ISCHYROCERIDAE (continúa). 

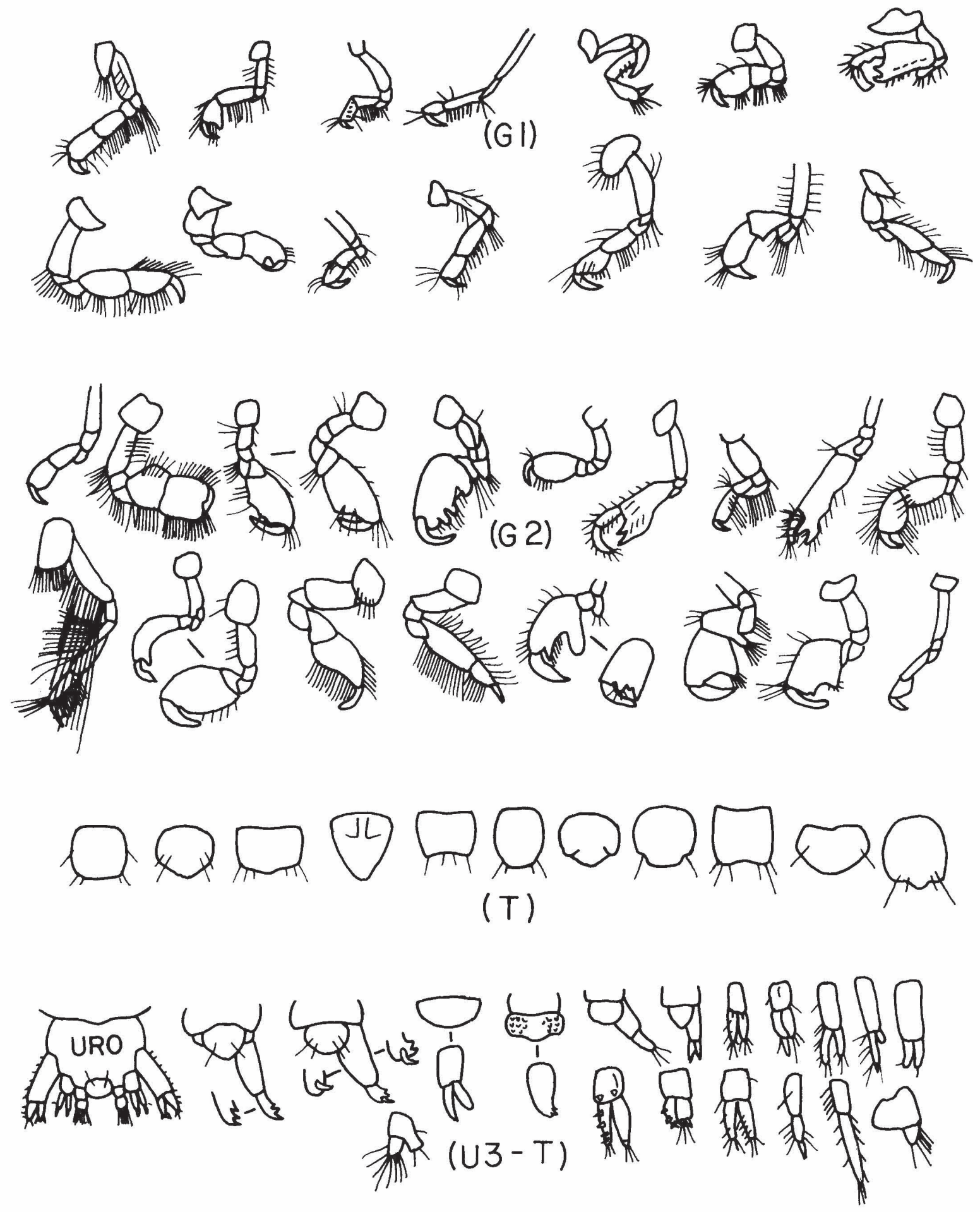

Lámina 23.- Familia COROPHIIDAE-ISCHYROCERIDAE (continúa). 

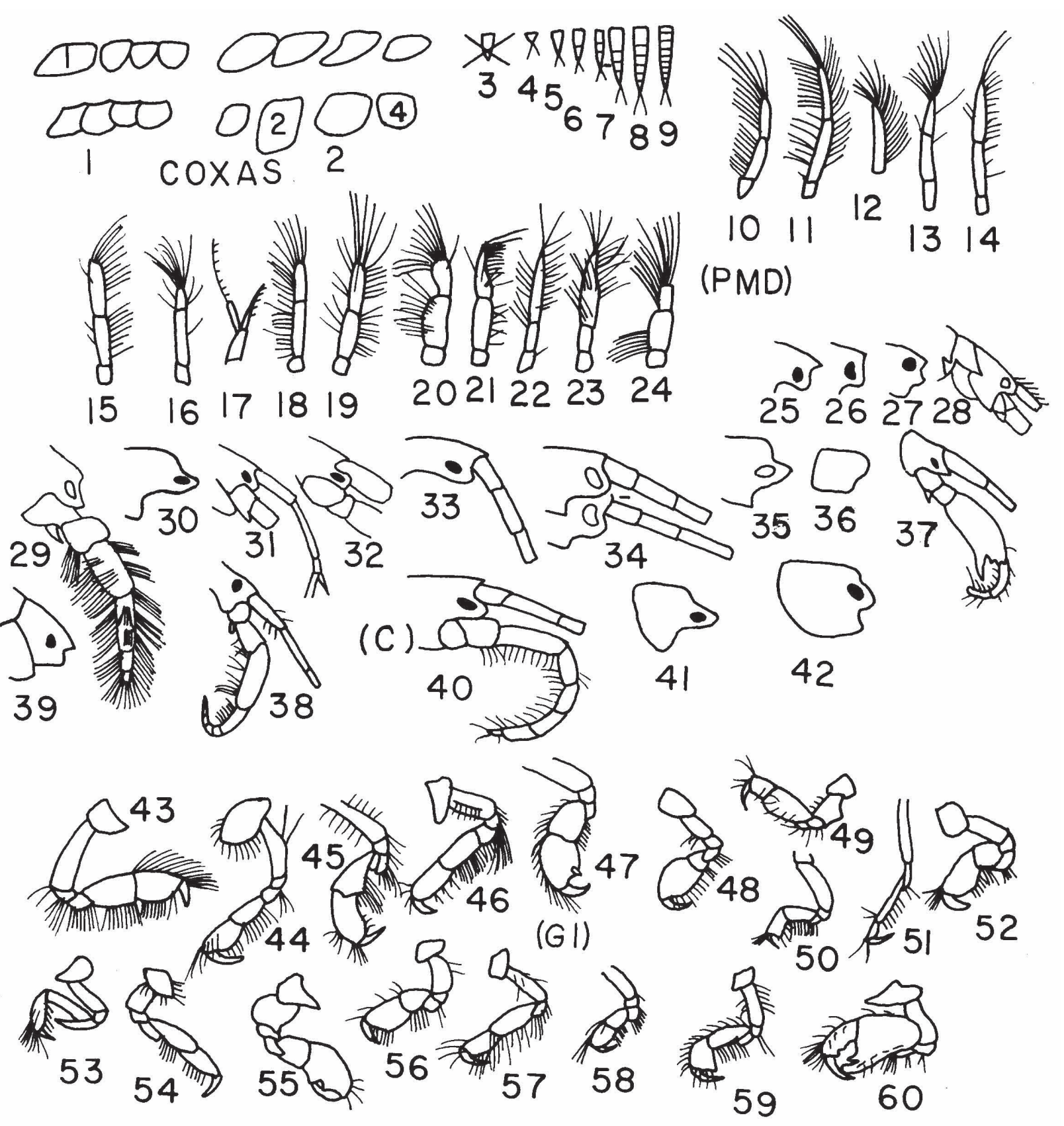

Lámina 24.- Familia COROPHIIDAE-ISCHYROCERIDAE (continúa). 

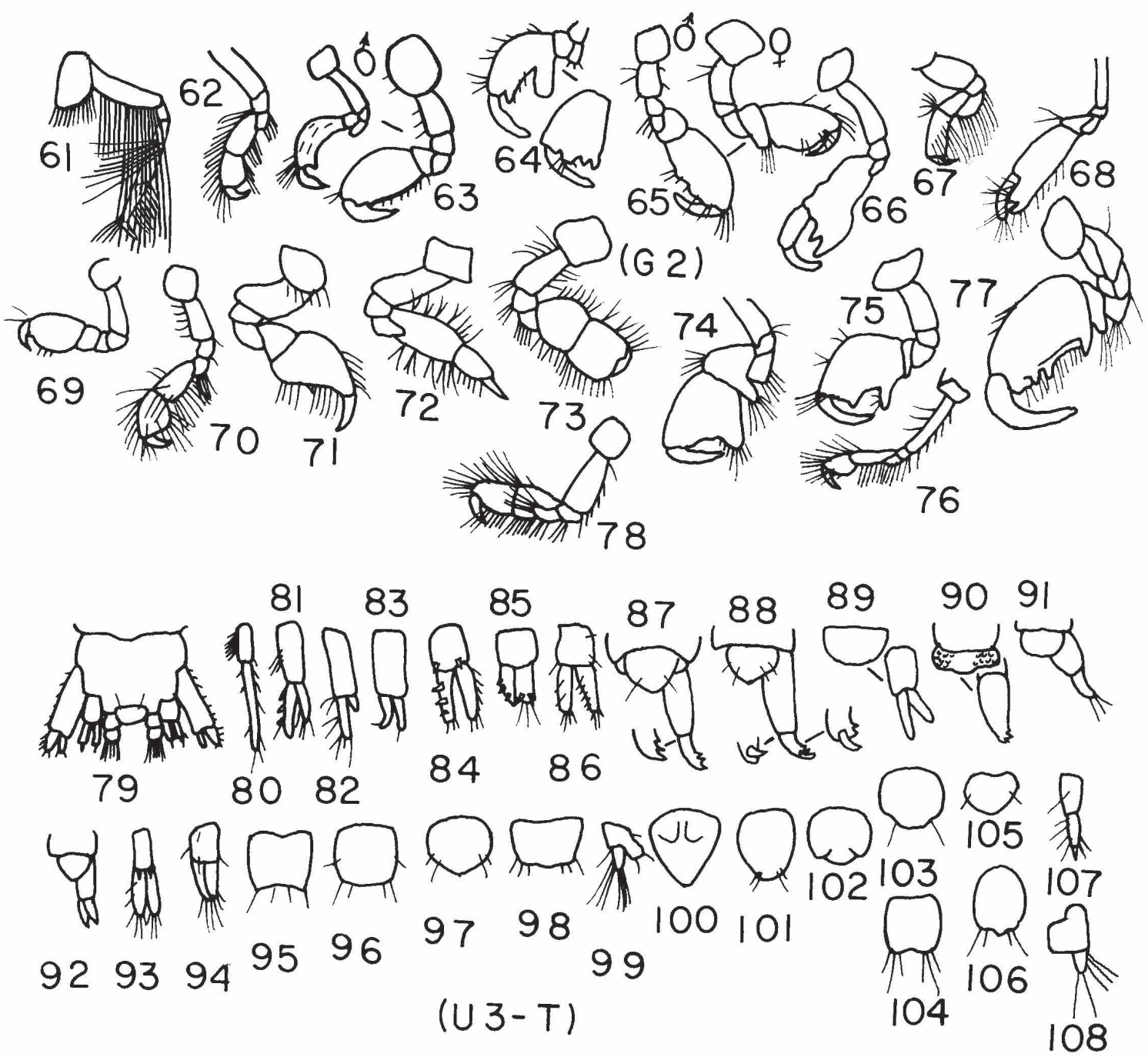

Lámina 25.- Familia COROPHIIDAE-ISCHYROCERIDAE (final).

1, 7, 14, 42, 53, 70, 93-94, $106 \Rightarrow$ Aora;

1, 4-5, 15, 36, 52, 69, $92 \Rightarrow$ Bonnierella;

1, 3, 16, 41, 51, 68, $91 \Rightarrow$ Cerapopsis;

2, 3, 17, 37, 50, 67, $79 \Rightarrow$ Corophium;

2, 3, 18, 38, 49, 66, $90 \Rightarrow$ Ericthonius;

1, 3-9, 19, 34, 57, 65, 89 $\Rightarrow$ Gammaropsis;

$1,6,20,33,58,63,87 \rightarrow$ Ischyrocerus;

1, 5, 24, 40, 48, 64, 88 • Jassa;

$1,7,21,25,47,62,86,104 \Rightarrow$ Lembos (sensu lato);

1, 6-7, 22, 26, 46, 61, 85, $103 \rightarrow$ Leptocheirus;

1, 5-6, 23, 60, 78, 84, $102 \Rightarrow$ Microdeutopus;

1, 4, 24, 27, 48, 64, 85, $106 \Rightarrow$ Microjassa;

1, 6, 13, 44, 77, 95-96, $107 \Rightarrow$ Microprotopus;

2, 8-9, 59, 76, 80, $100 \rightarrow$ Neohela;

$1,4,24,29,56,75,83,101 \Rightarrow$ Parajassa;

1, 3-4, 10, 45, 74, 82, $98 \Rightarrow$ Photis;

1, 7-9, 31, 43, 73, $81 \Rightarrow$ Protomedeia;

2, 12, 32, 54, 71, 105, $108 \Rightarrow$ Siphonoecetes;

2, 6-7, 38, 55, 72, 97, $99 \Rightarrow$ Unciola. 


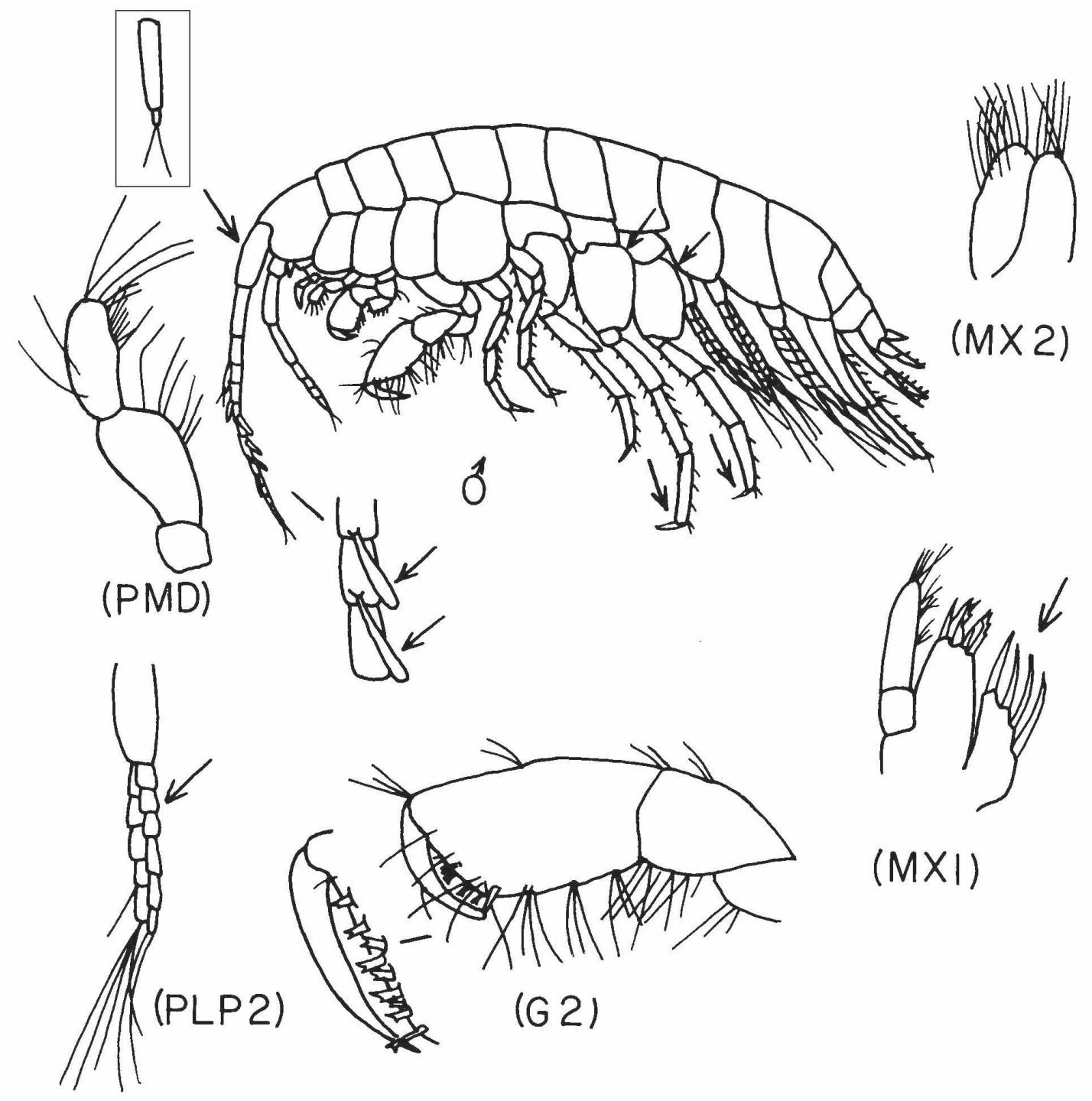

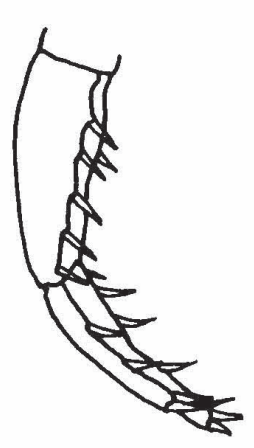

(UI)

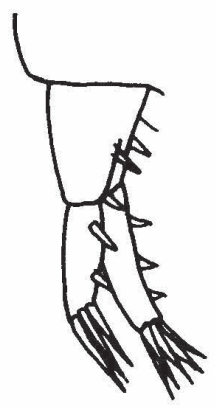

(U2)

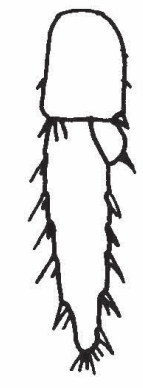

(U3)

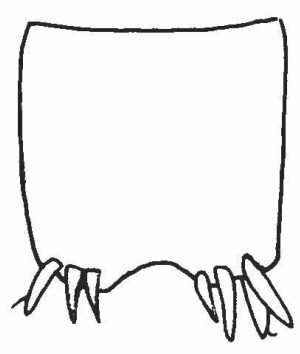

( T )

Lámina 26.- Familia CRANGONYCTIDAE (un solo género en el área) — Crangonyx. (ver GAMMARIDAE-MELITIDAE y METACRANGONYCTIDAE). 

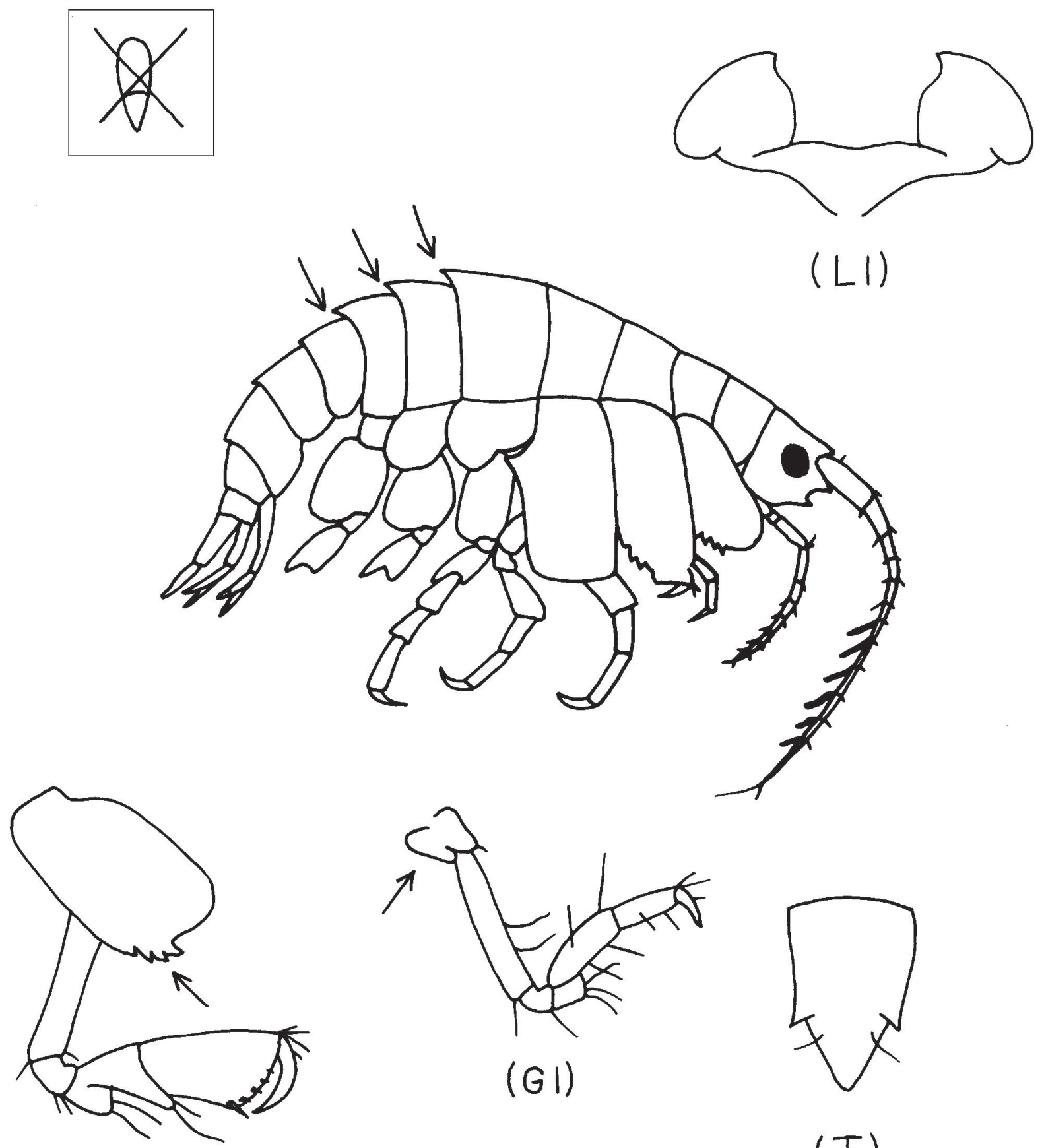

(GI)

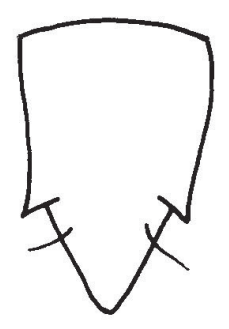

(G 2)

$(T)$

Lamina 27.- Familia CRESSIDAE (un solo género en el área) $\Rightarrow$ Cressa. 

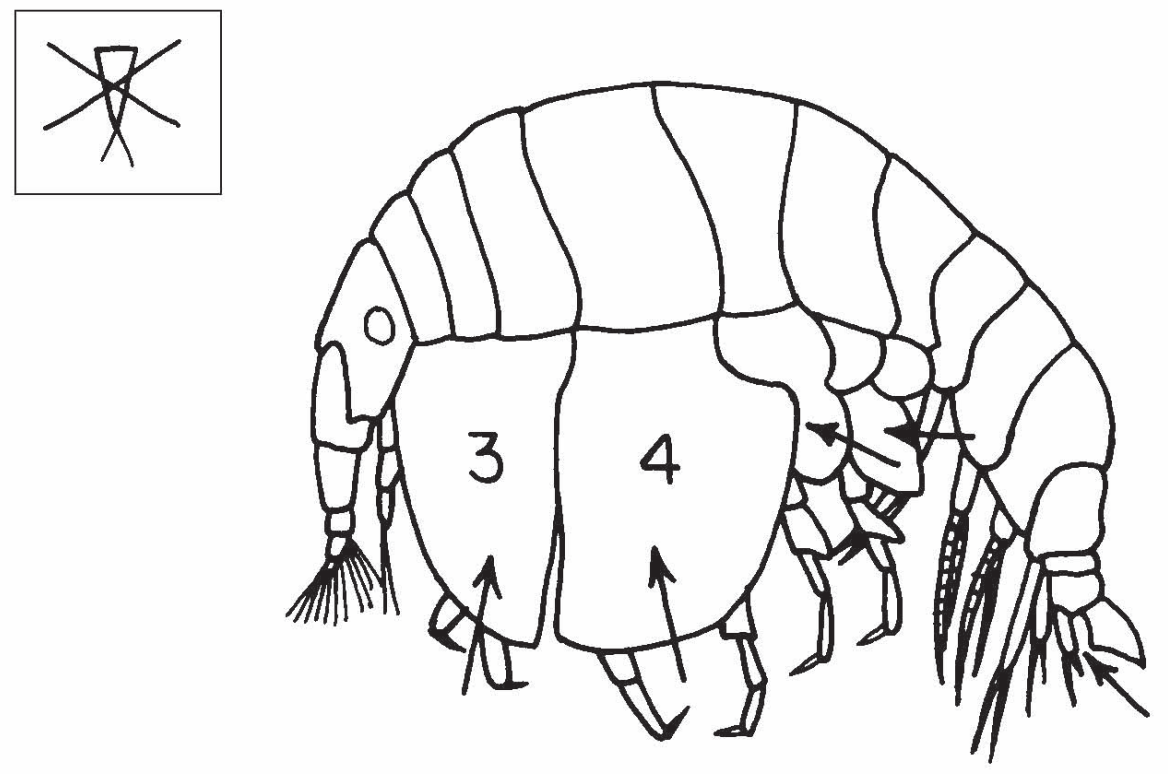

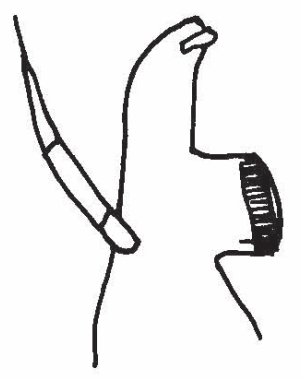

(MD)

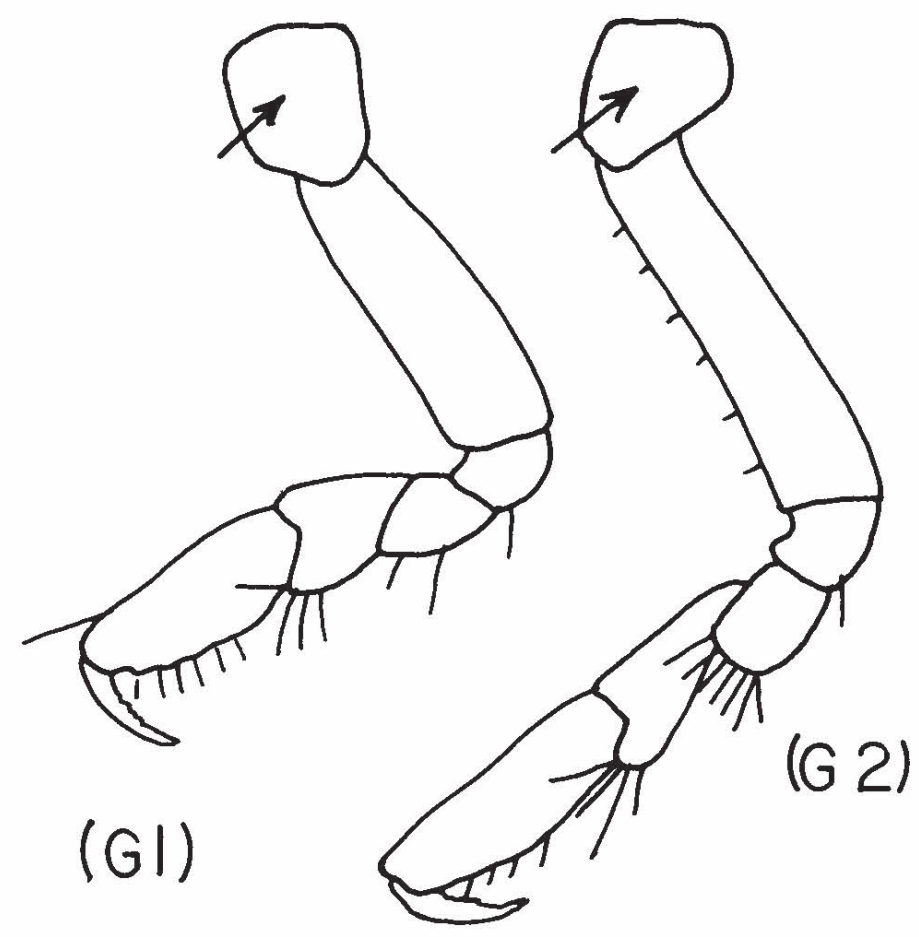

Lámina 28.- Familia CYPROIDEIDAE (un solo género en el área) $\rightarrow$ Peltocoxa (ver AMPHILOCHIDAE). 

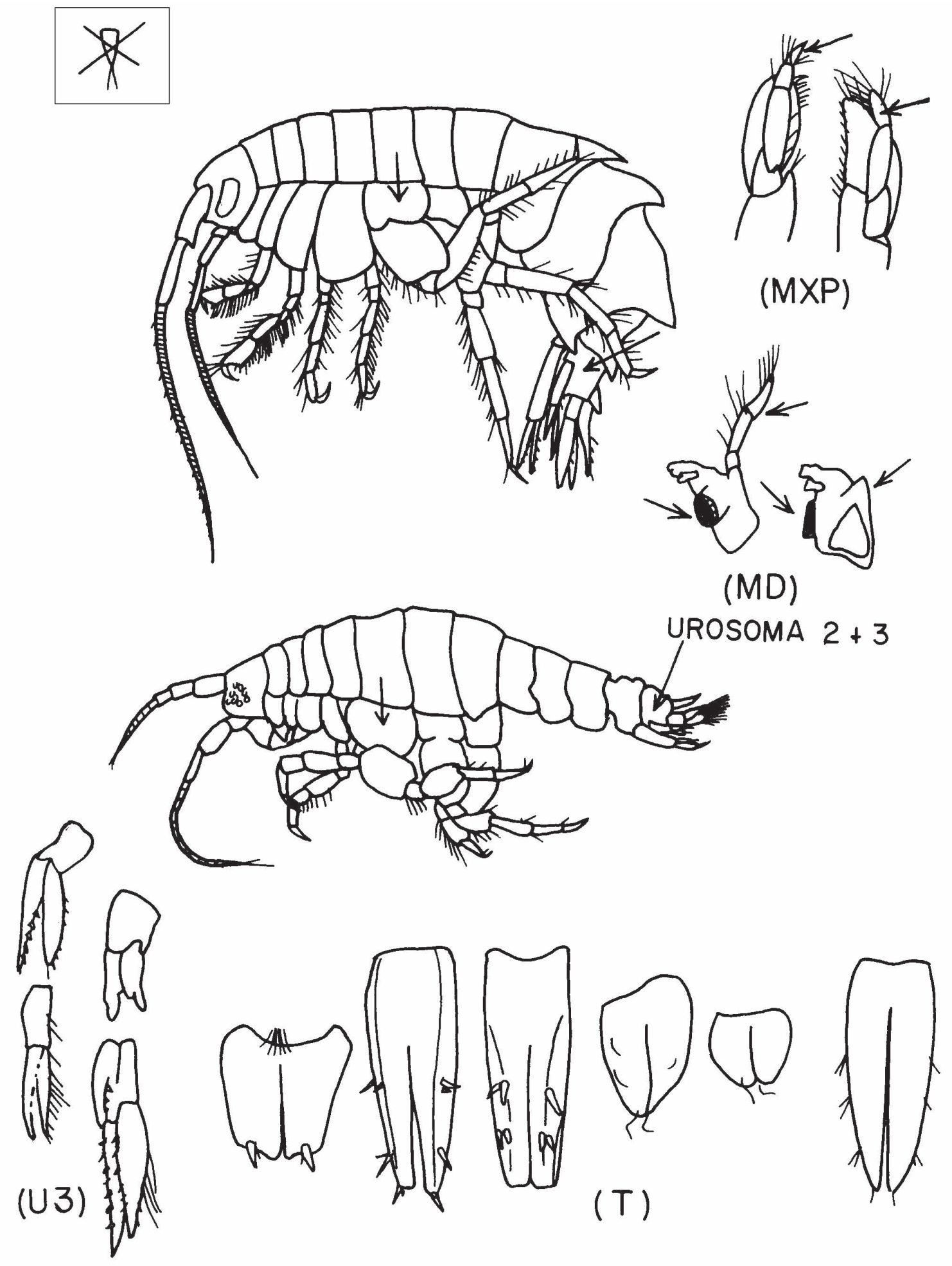

Lámina 29.— Familia DEXAMINIDAE (continúa). 

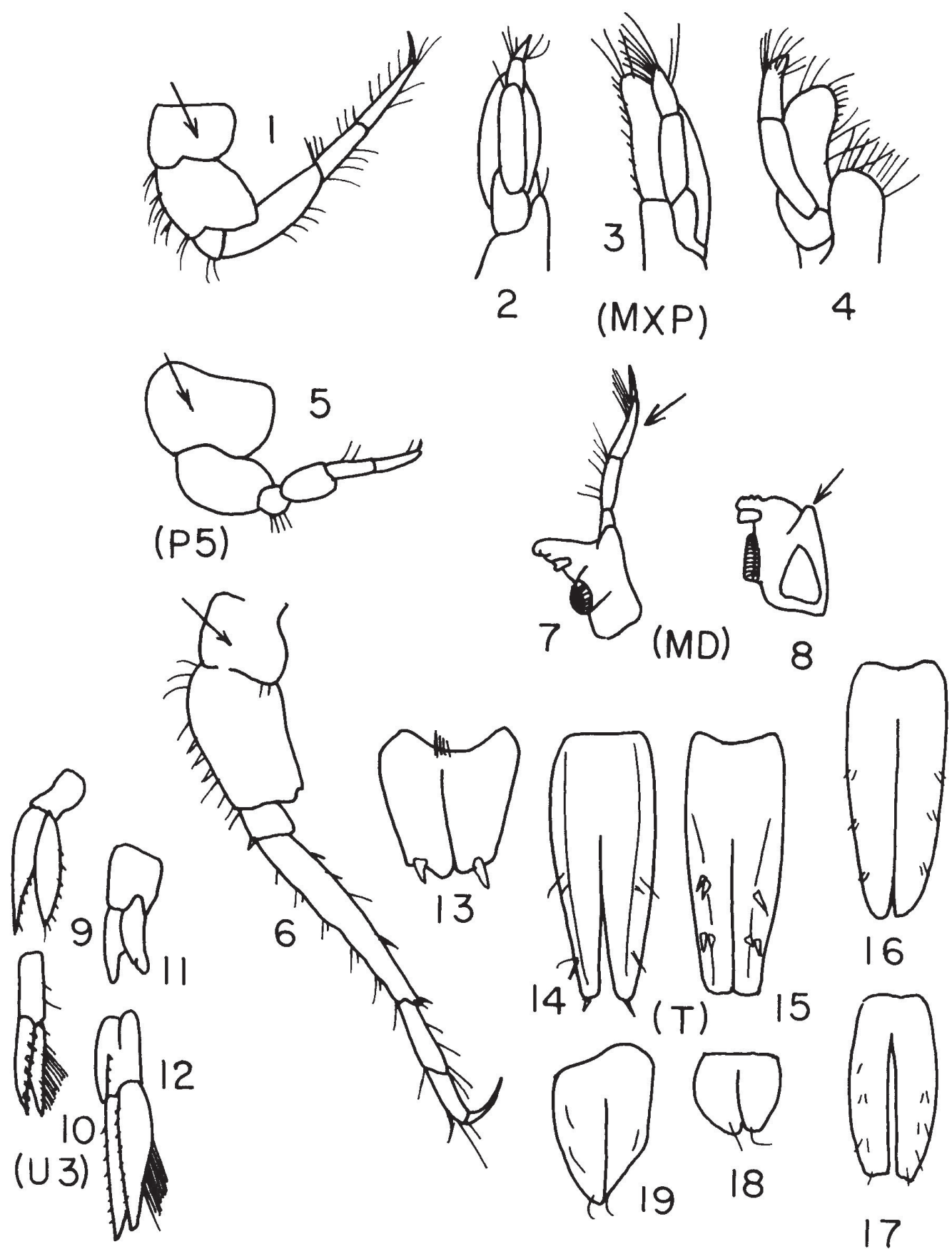

Lámina 30.- Familia DEXAMINIDAE (final).

1, 2-3, 7, 9-10, $13 \Rightarrow$ Atylus;

2, 5, 8, 11, 18-19 $\Rightarrow$ Guernea;

1, 3, 8, 9-10, 14-15 $\Rightarrow$ Dexamine;

4, 6, 8, 12, 16-17 $\Rightarrow$ Tritaeta. 


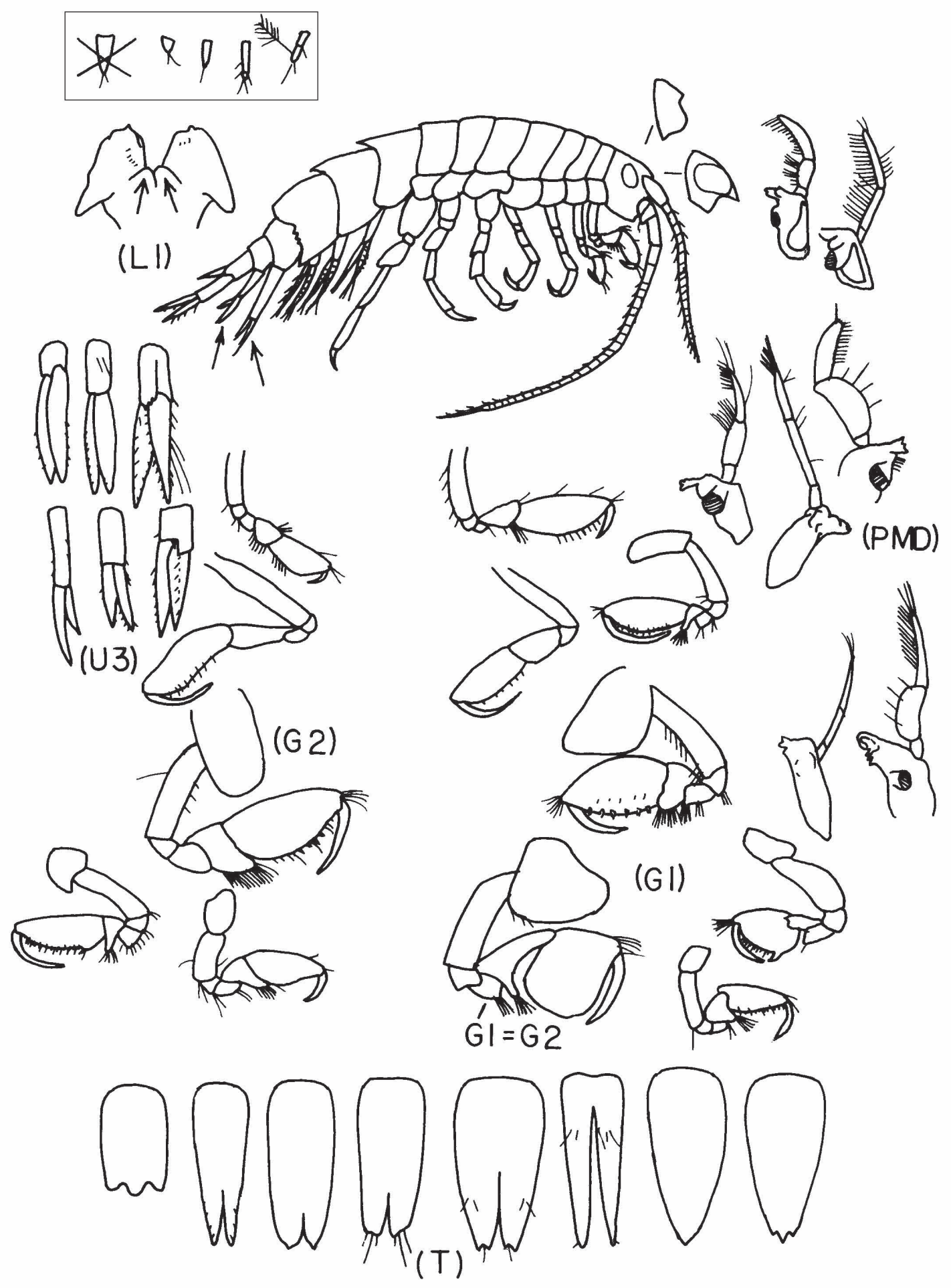

Lámina 31.- Familila EUSIRIDAE (continúa). 

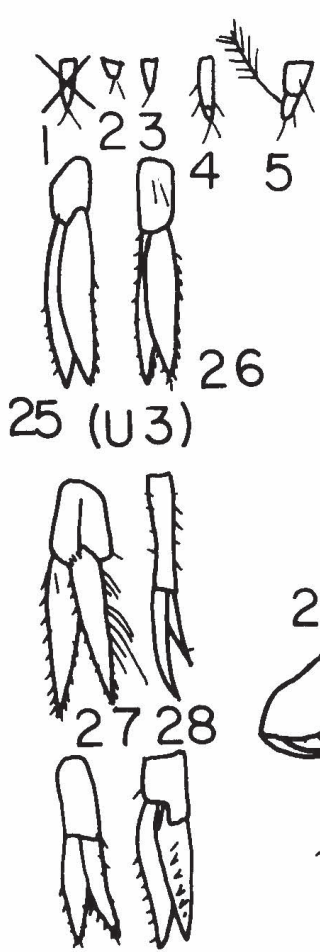

2930

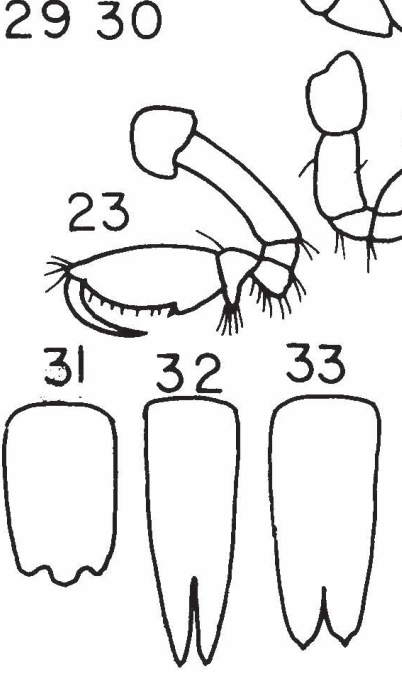

21

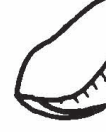

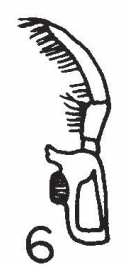
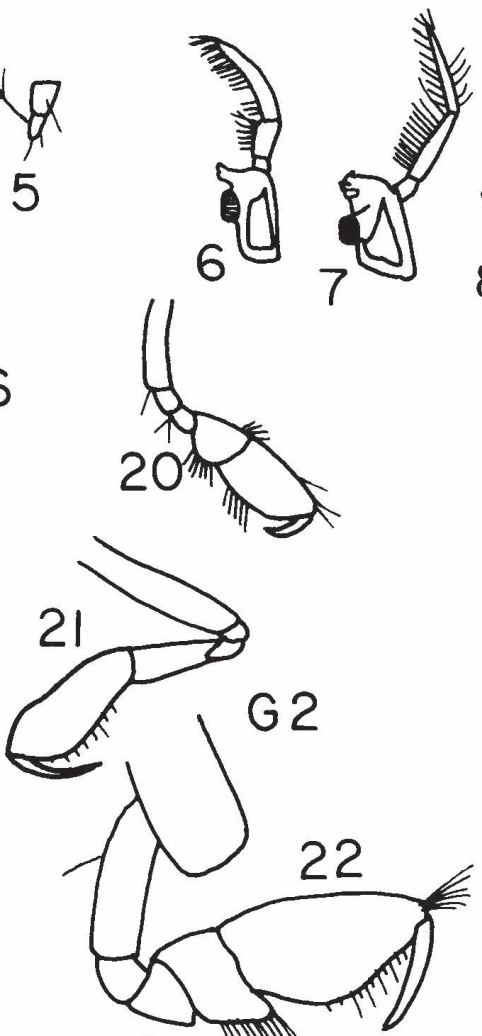

24
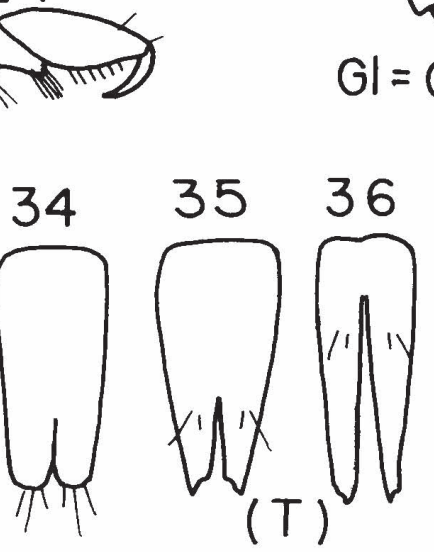
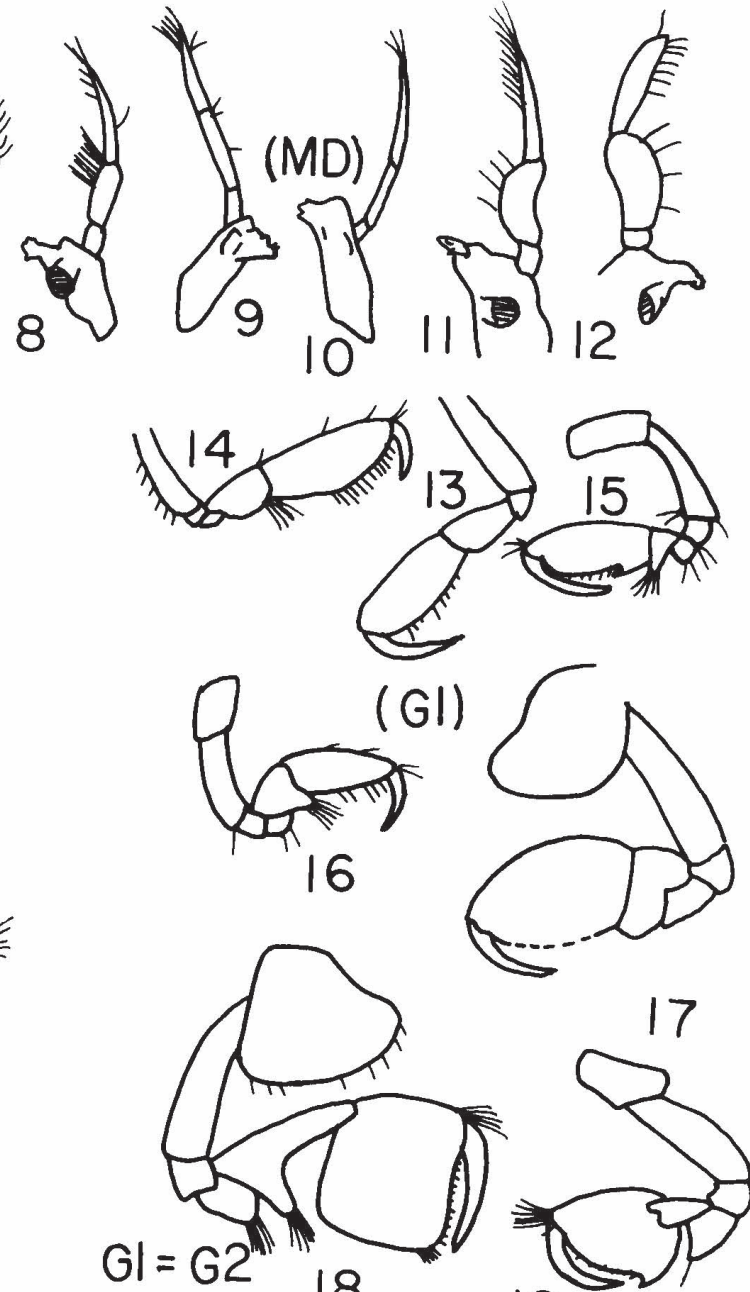

19

Lámina 32. - Familila EUSIRIDAE (final).

1, 8, 16, 20, 29, 37-38 Apherusa;

2, 9, 13, 21, 28, $36 \Rightarrow$ Eusirella;

3, 11, 17, 22, 30, $35 \Rightarrow$ Eusiroides;

2, 10, 19, $34 \Rightarrow$ Eusiropsis;

4, 7, 18, 27, $33 \Rightarrow$ Eusirus;

$5,6,15,23,26,32 \rightarrow$ Rhachotropis;

1, 12, 16, 24, 25, $31 \Rightarrow$ Stenopleura. 

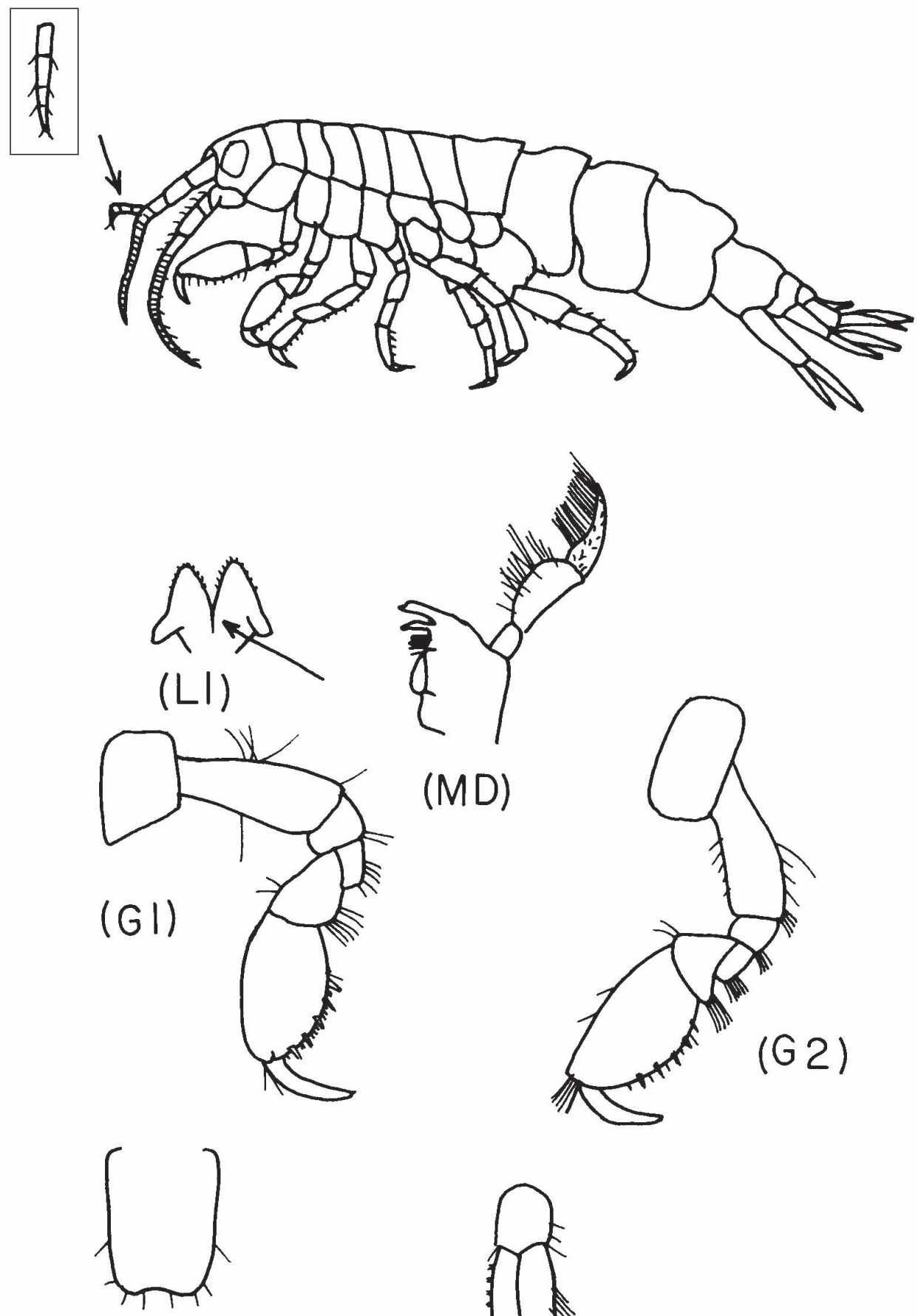

( $T$ )

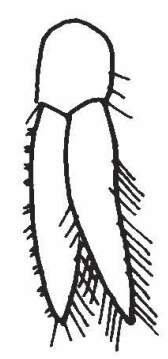

(U 3)

Lámina 33.—Familia GAMMARELLIDAE (monogenérica) * Gammarellus. 


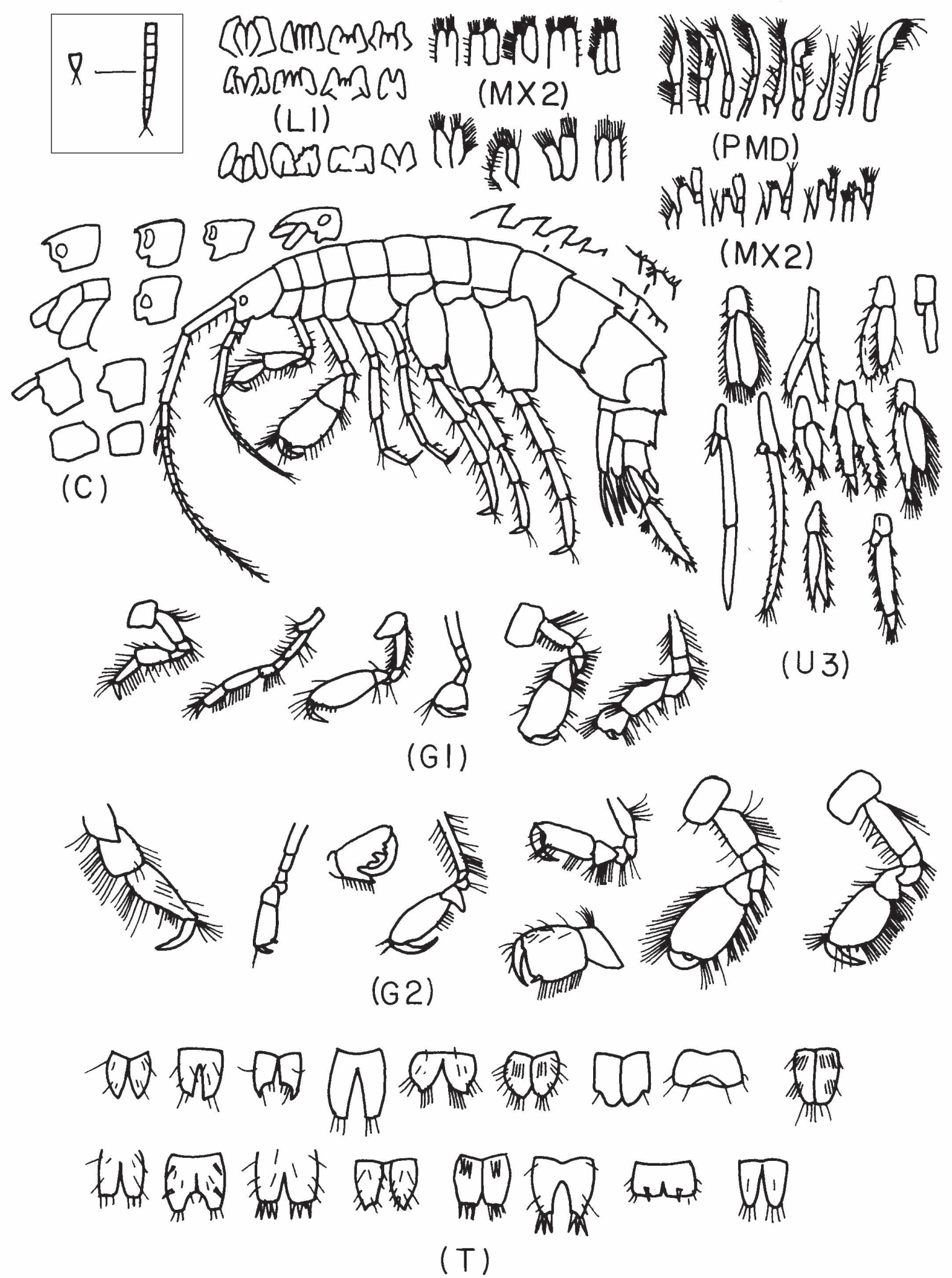

Lámina 34.— Familia GAMMARIDAE-MELITIDAE (continúa). 


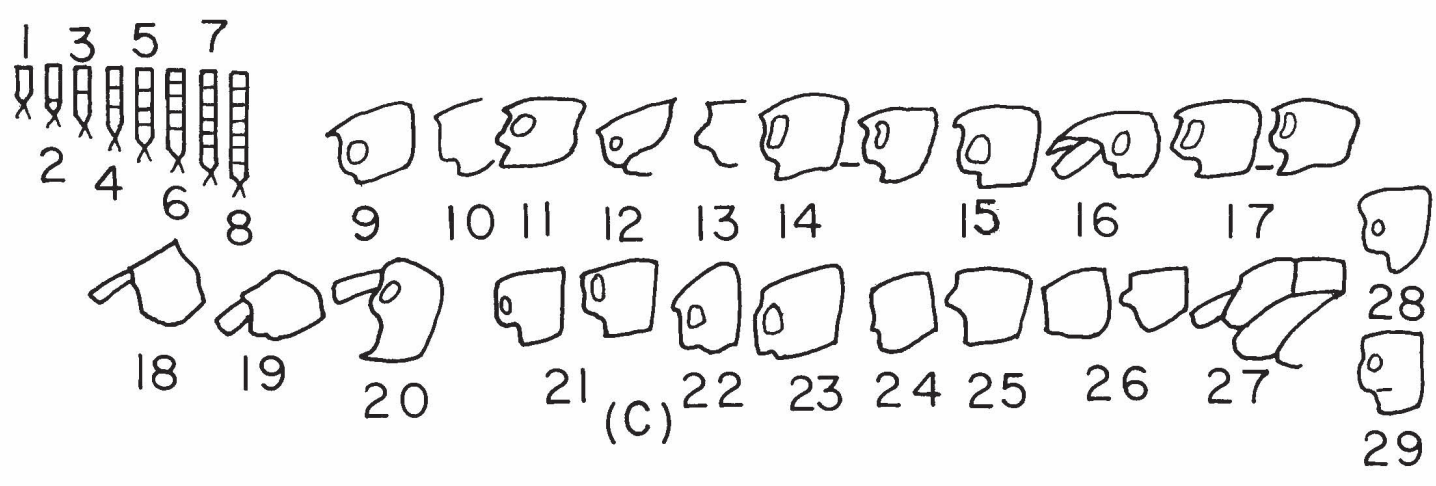

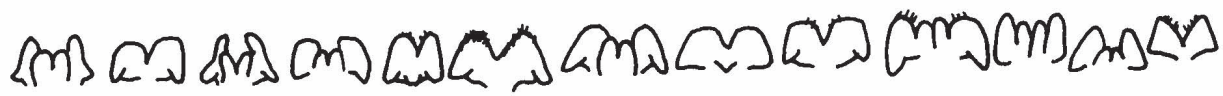

$\begin{array}{lllllllllllll}30 & 31 & 32 & 33 & 34 & 35 & 36 & 37 & 38 & 39 & 40 & 41 & 42\end{array}$

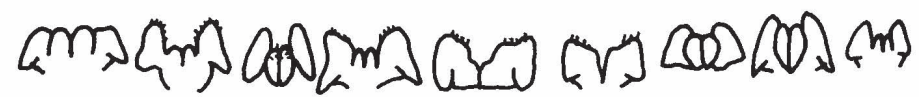

$43 \quad 44 \quad 4546 \quad 47(1) 48495051$

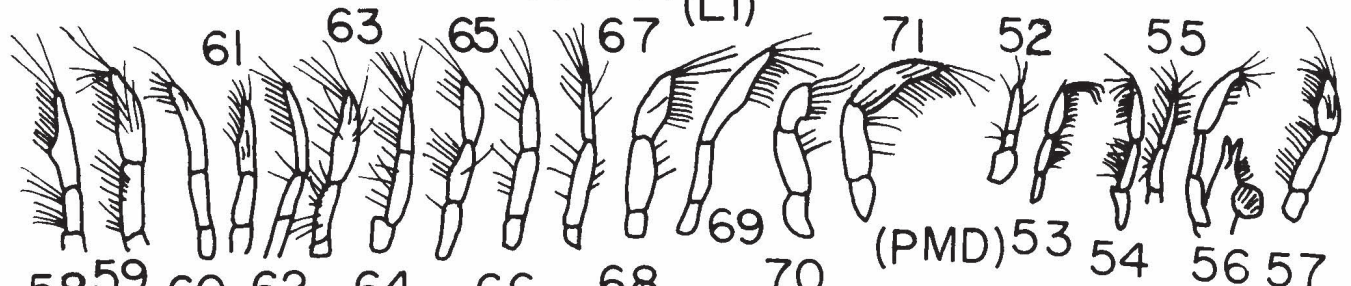

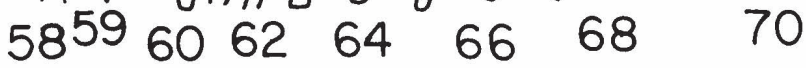

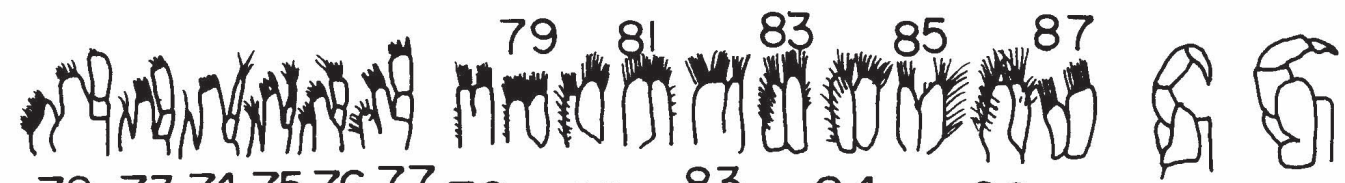

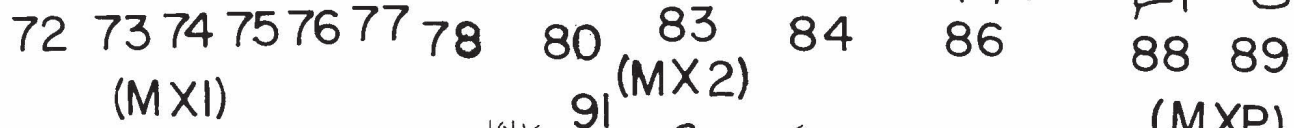

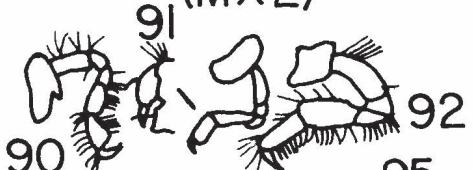

$(\mathrm{MXP})$

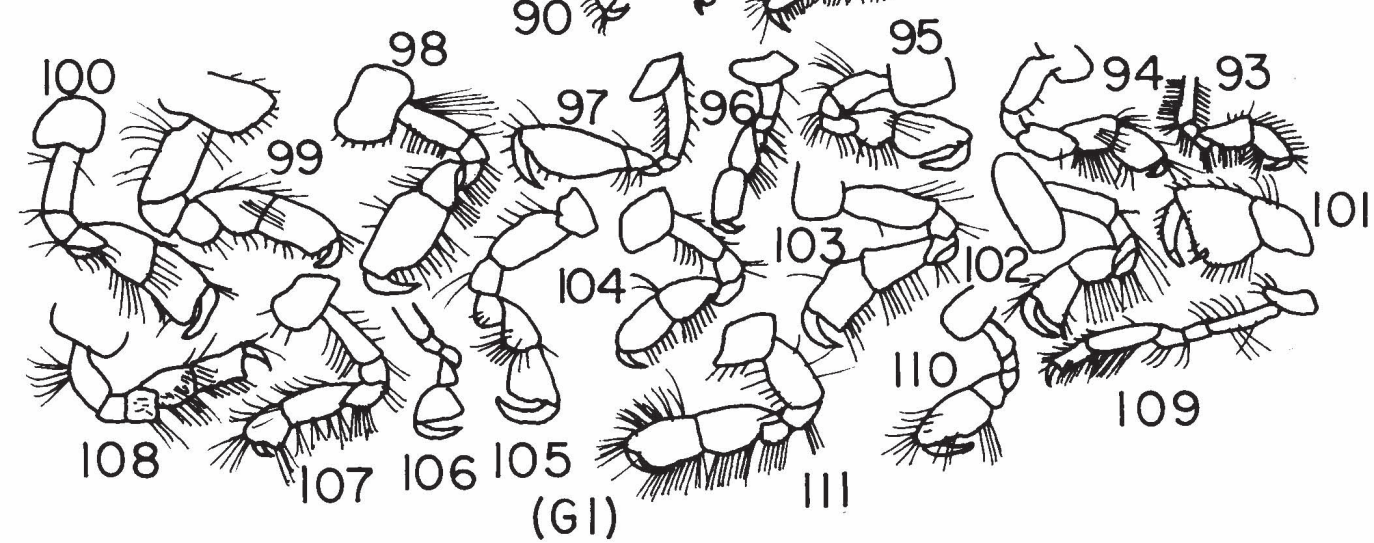

Lámina 35.- Familia GAMMARIDAE -MELITIDAE (continúa). 

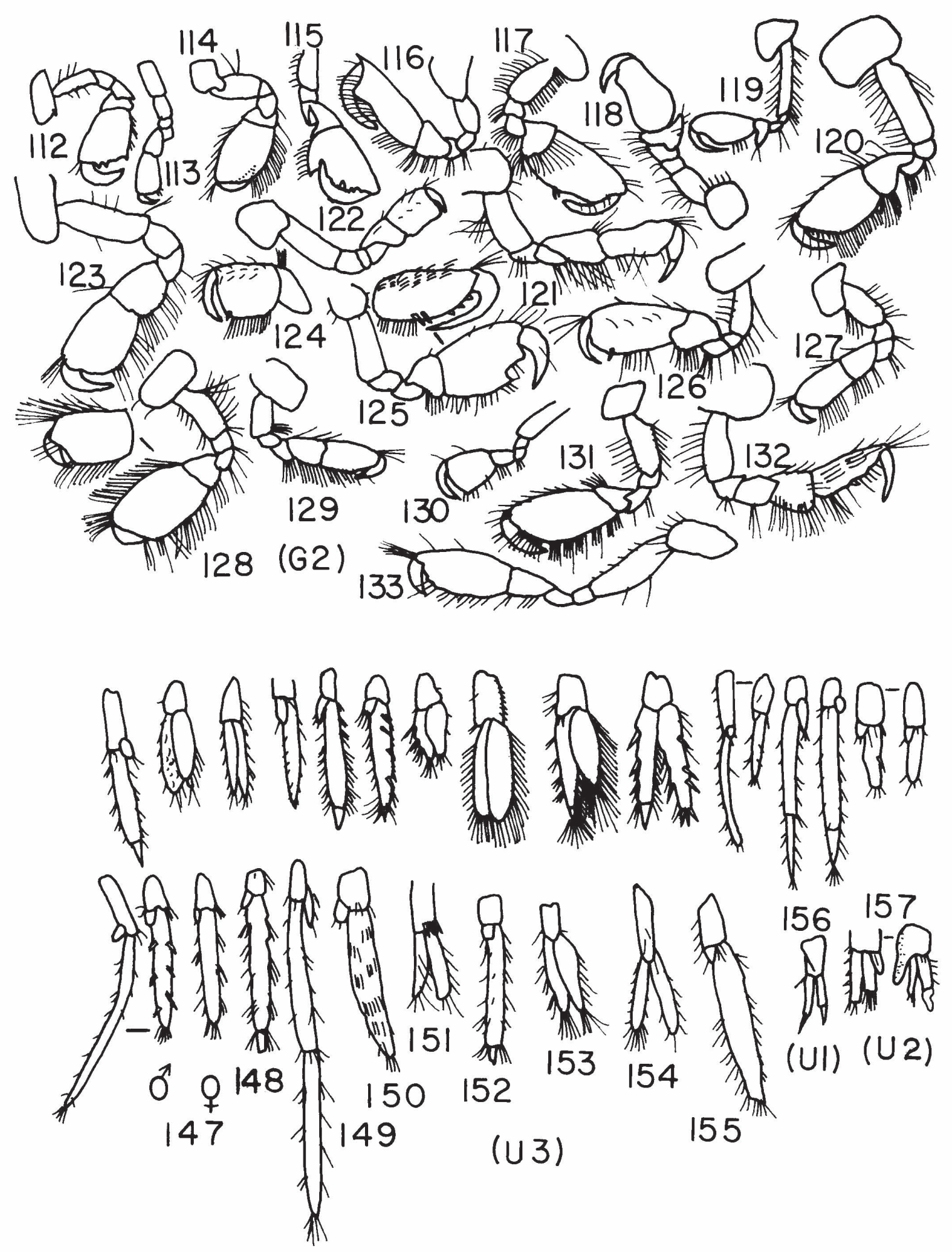

Lámina 36. - Familia GAMMARIDAE-MELITIDAE (continúa). 

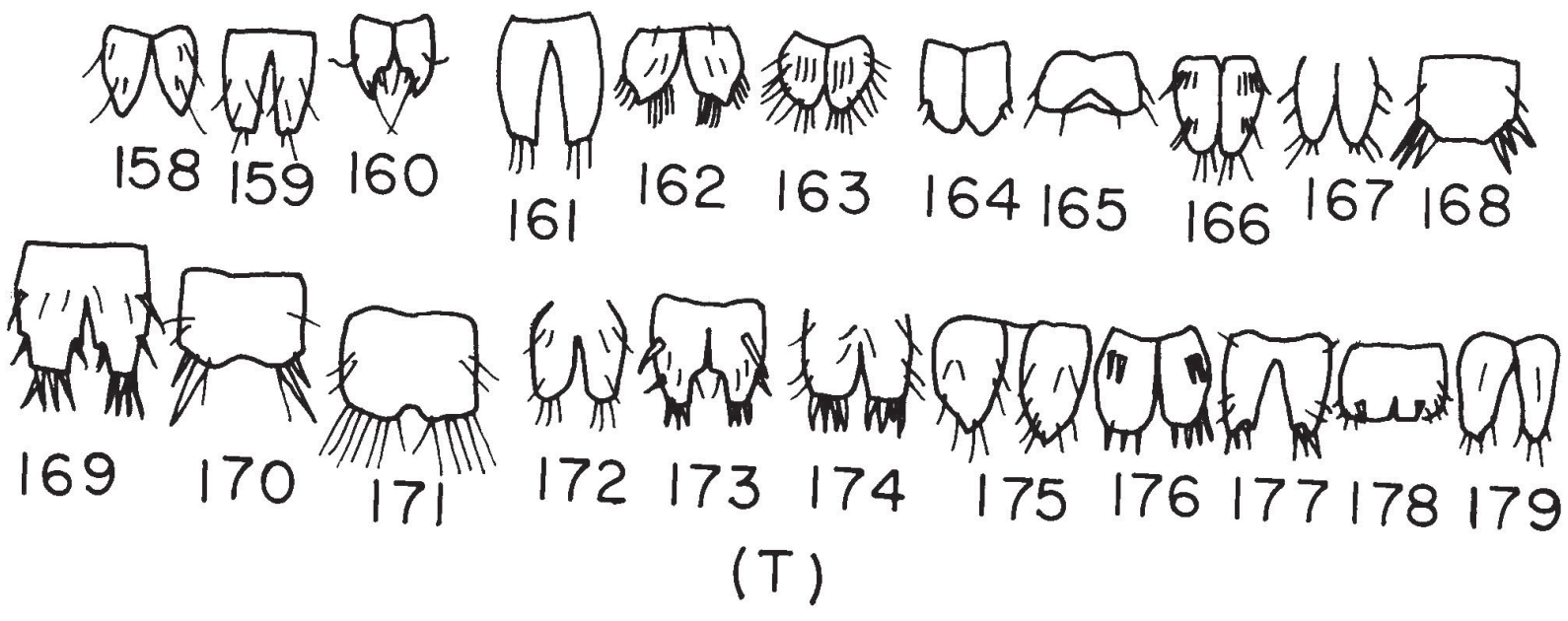

Lámina 37.- Familia GAMMARIDAE-MELITIDAE (final)

2-4, 9, 30, 52, 72, 80, 90, 112, 134, $158 \rightarrow$ Abludomelita;

$7,10,31,53,91,113,135,159 \Rightarrow$ Bathyceradocus

2-3, 11, 32, 54, 92, 114, 136, $160 \Rightarrow$ Cheirocratus;

$5,12,33,55,77,84,93,115,137,161 \Rightarrow$ Dulichiella;

2, 13, 34, 56, 72, 86, 94, 116, 138, $162 \rightarrow$ Dulzura;

1-7, 14, 35, 57, 72, 80, 95, 117, 139, 163 Echinogammarus;

2-4, 15, 36, 58, 73, 81, 96, 118, 140, $164 \Rightarrow$ Elasmopus;

$4,16,37,73,80,97,119,141,165,180 \Rightarrow$ Gammaracanthus;

$3-7,17,38,59,72,80,98,120,142,166 \Rightarrow$ Gammarus;

2, 18, 46, 61, 73, 78, 106, 130, 150, $174 \rightarrow$ Haploginglymus;

2-3, 19, 47, 62, 72, 85, 89, 108, 132, 151, $175 \Rightarrow$ Liagoceradocus;

$4-5,48,63,73,83,110,129,152,176 \rightarrow$ Lusigammarus;

5-8, 21, 49, 64, 76, 81, 111, 131, 153, $177 \Rightarrow$ Maera;

6-7, 50, 65, 73, 87, 109, 133, 154, $178 \Rightarrow$ Maerella;

3-5, 23, 41-42, 66, 77, 79, 107, 128, 155, $179 \Rightarrow$ Melita;

2, 24, 38, 60, 72, 80, 88, 99, 121, 143, 157, $167 \Rightarrow$ Metahadzia;

2, 25, 38-39, 68, 73, 82, 100, 122, 144, $168 \Rightarrow$ Metaniphargus;

3, 26, 40-41, 69, 74, 82, 101, 124, 145, $169 \Rightarrow$ Niphargus;

$2,27,51,70,73,82,102,126,146,170 \Rightarrow$ Parapseudoniphargus;

3, 28, 44-45, 67, 72, 84, 105, 125, 149, 173 \& Psammogammarus;

2, 25, 43, 71, 73, 82, 103, 123, 147, $171 \Rightarrow$ Pseudoniphargus;

4-5, 29, 83, 104, 127, 148, 156, $172 \Rightarrow$ Riphidogammarus;

(ver CRANGONYCTIDAE, GAMMARELLIDAE, MEGALUROPIDAE, METACRANGONYCTIDAE y NUUANIDAE). 

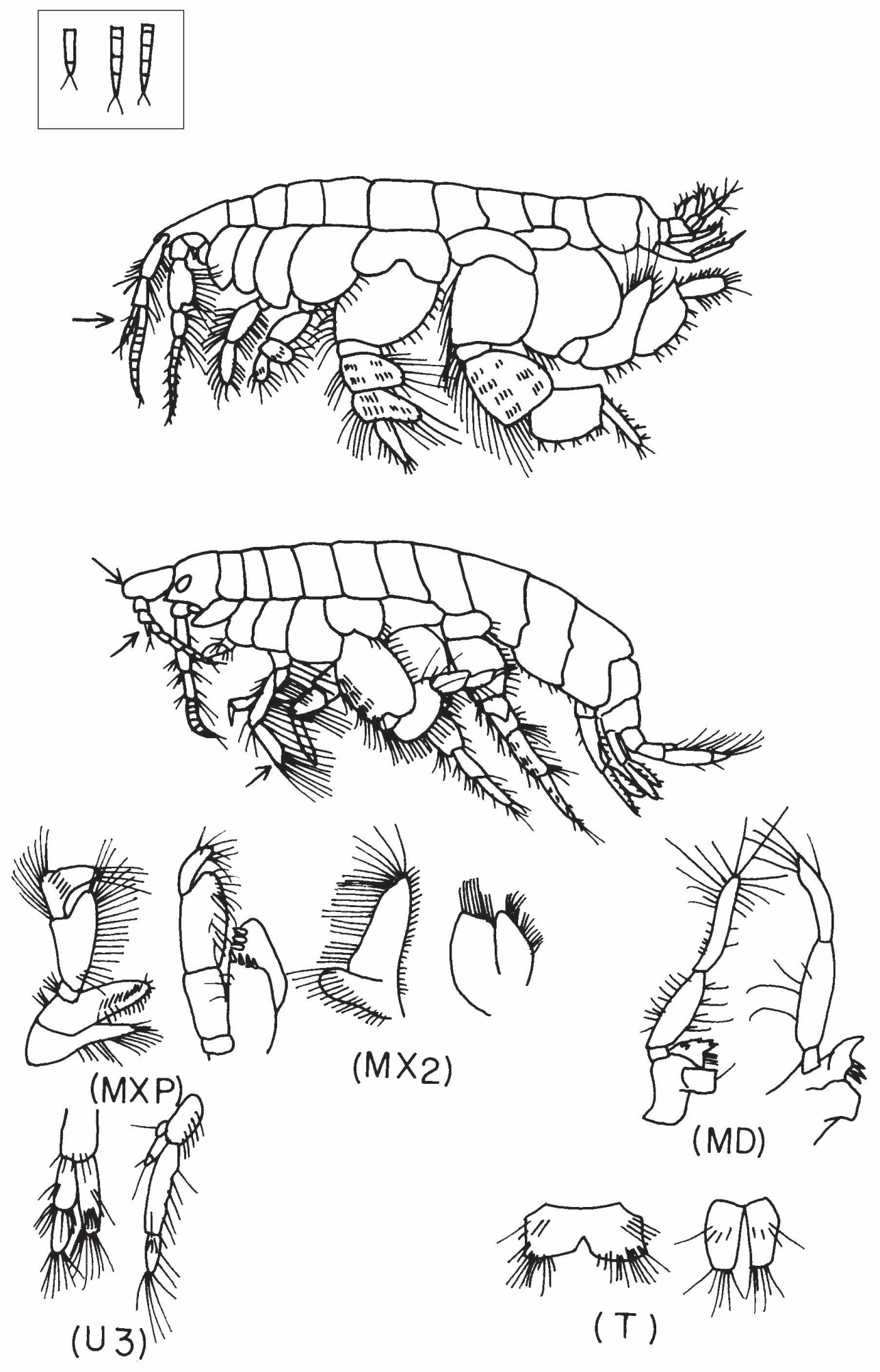

Lámina 38.- Familia HAUSTORIIDAE (continúa). 

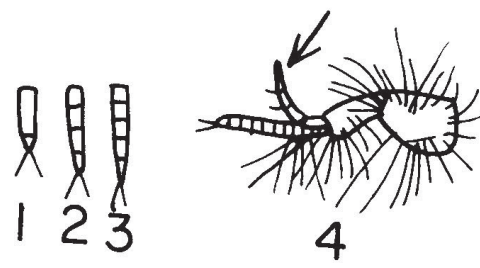

4
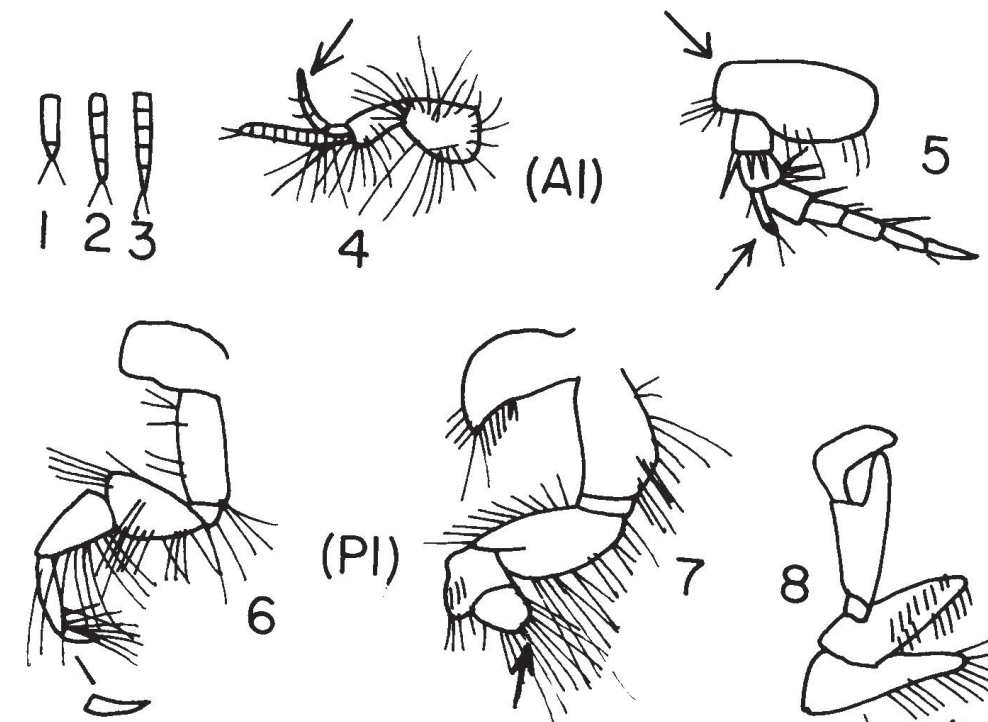

(PI)
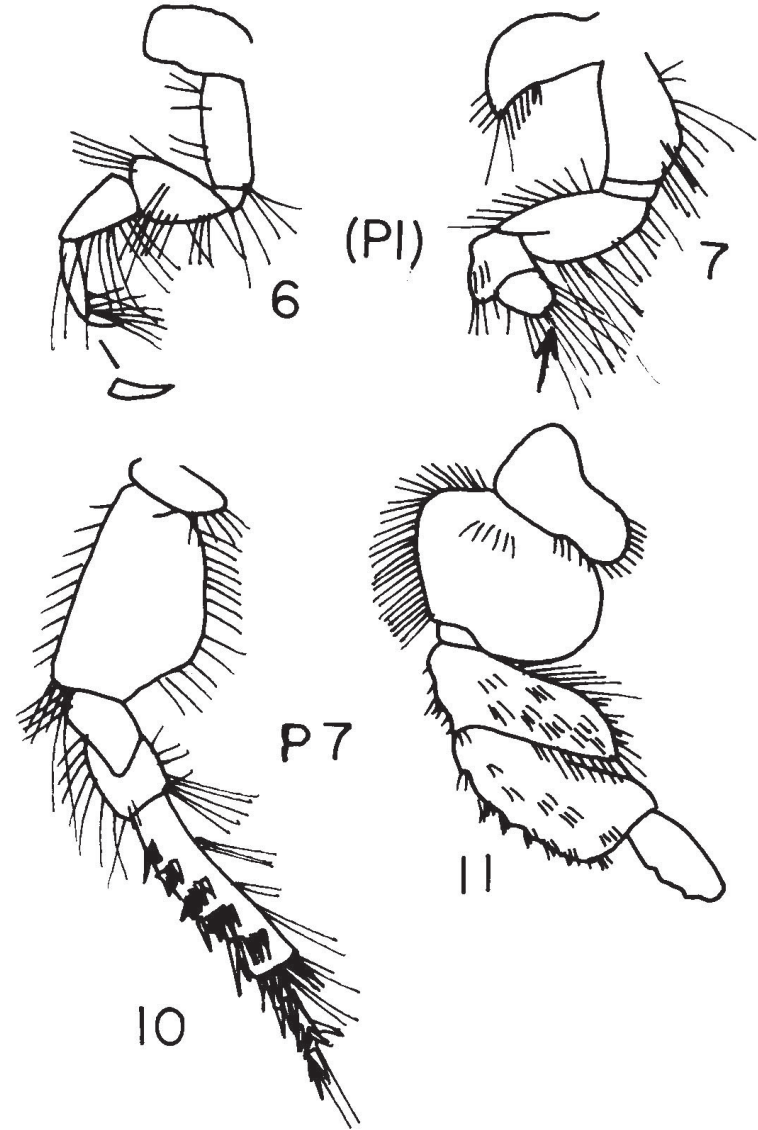

(AI)

6
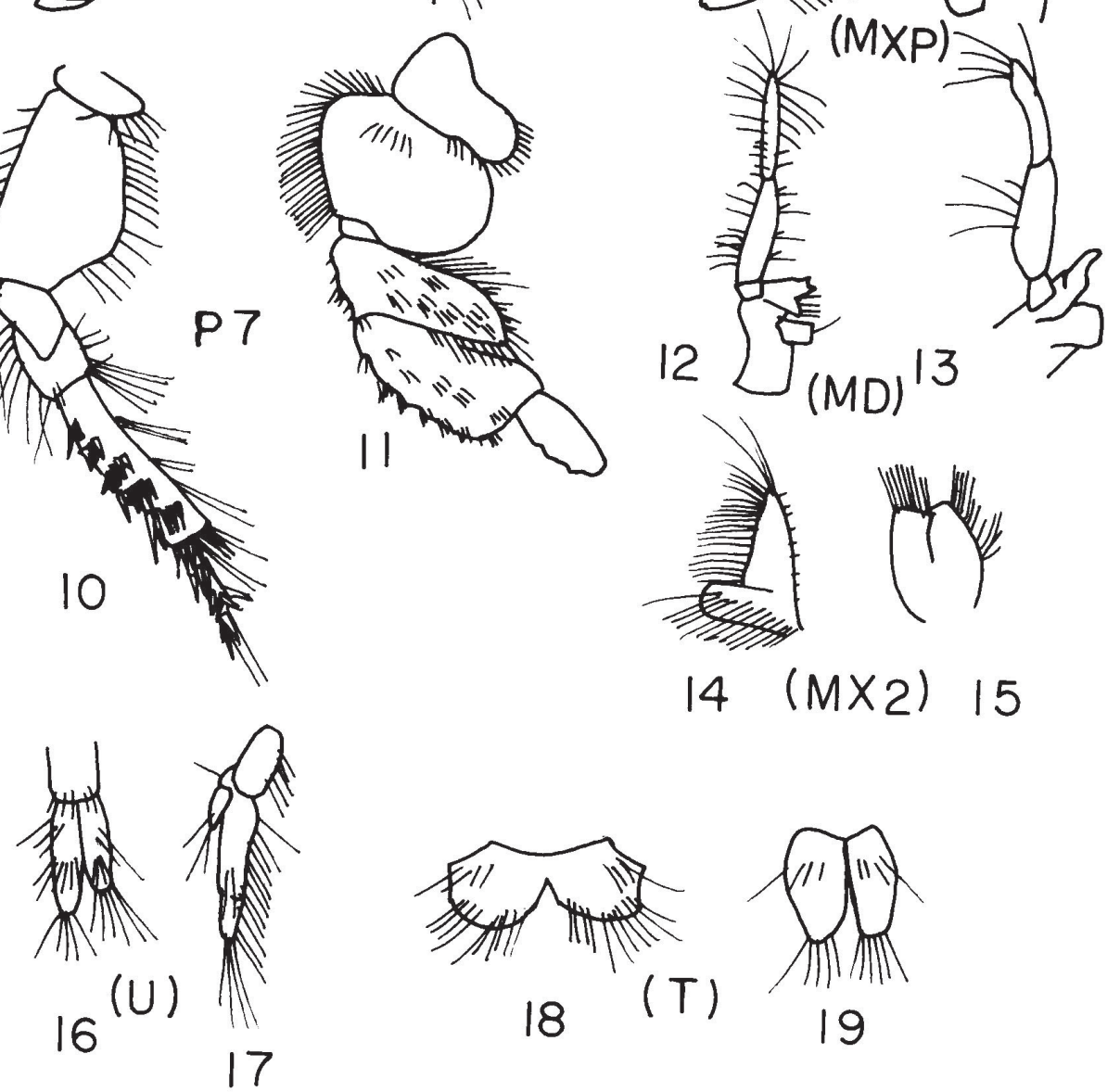

Lámina 39.- Familia HAUSTORIIDAE (final).

1, 5, 6, 9, 10, 13, 15, 17, $19 \Rightarrow$ Bathyporeia;

2-3, 4, 7, 8, 11, 12, 14, 16, $18 \rightarrow$ Haustorius. 

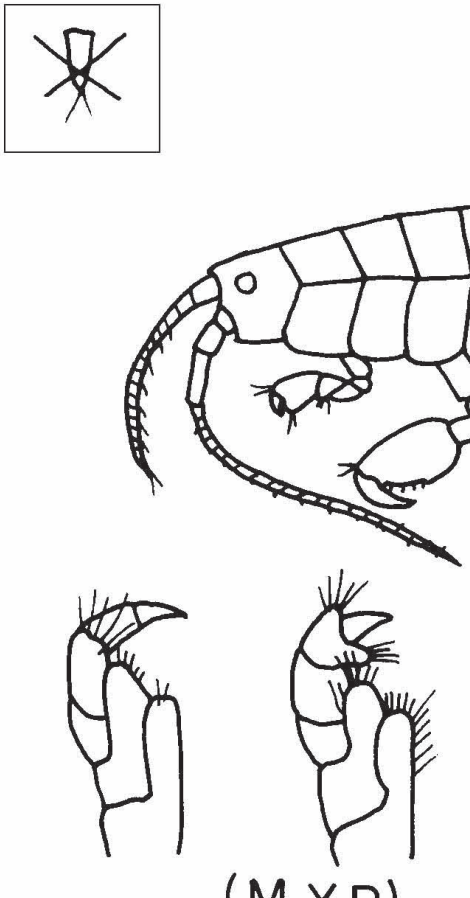

$(M \times P)$
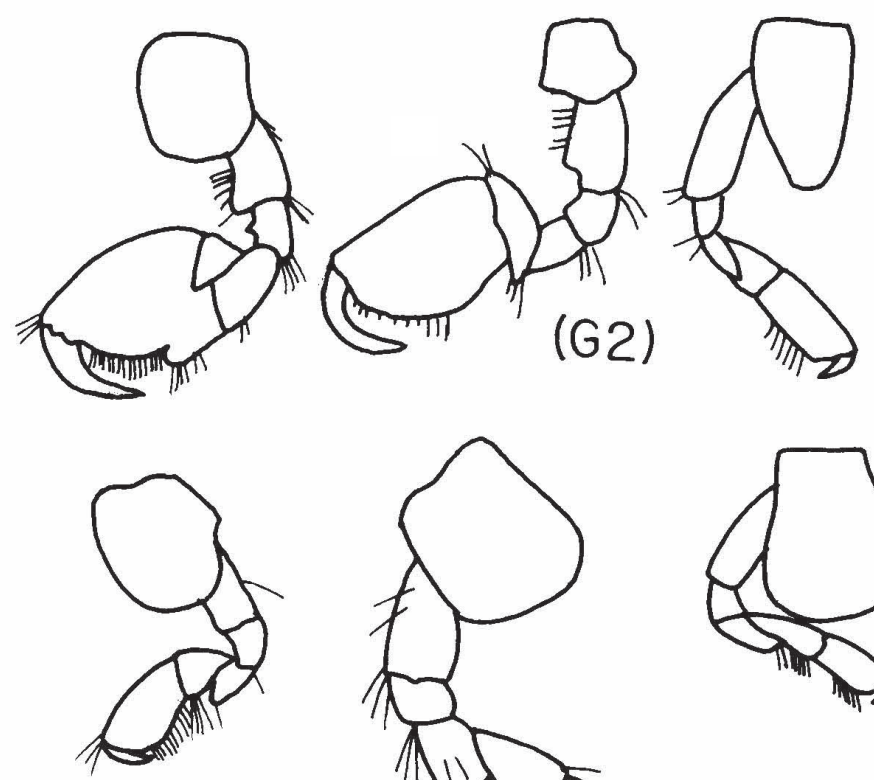

(G I)

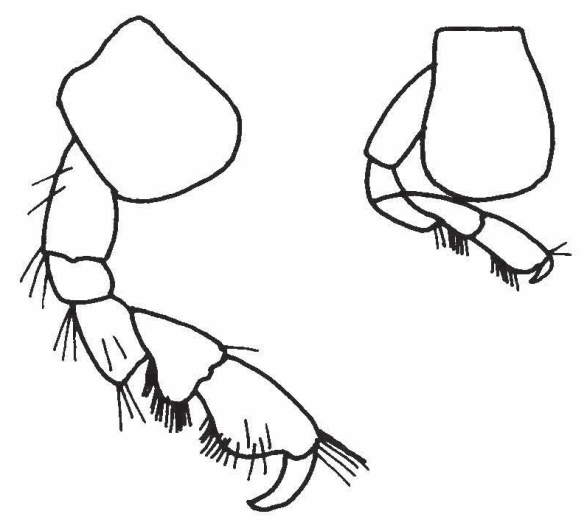

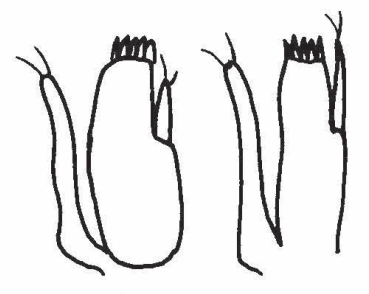

$(M \times 1)$
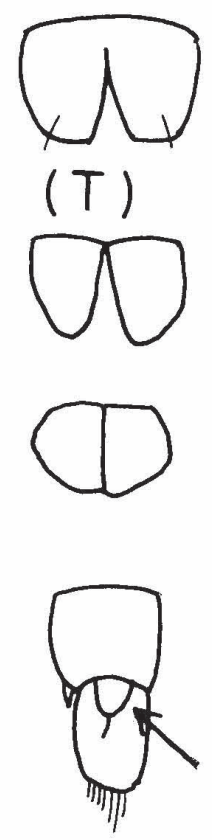

(U3)

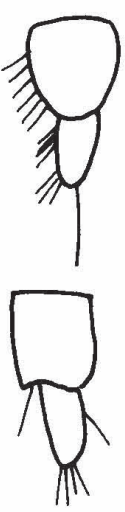

Lámina 40.— Familia HYALIDAE (continúa). 

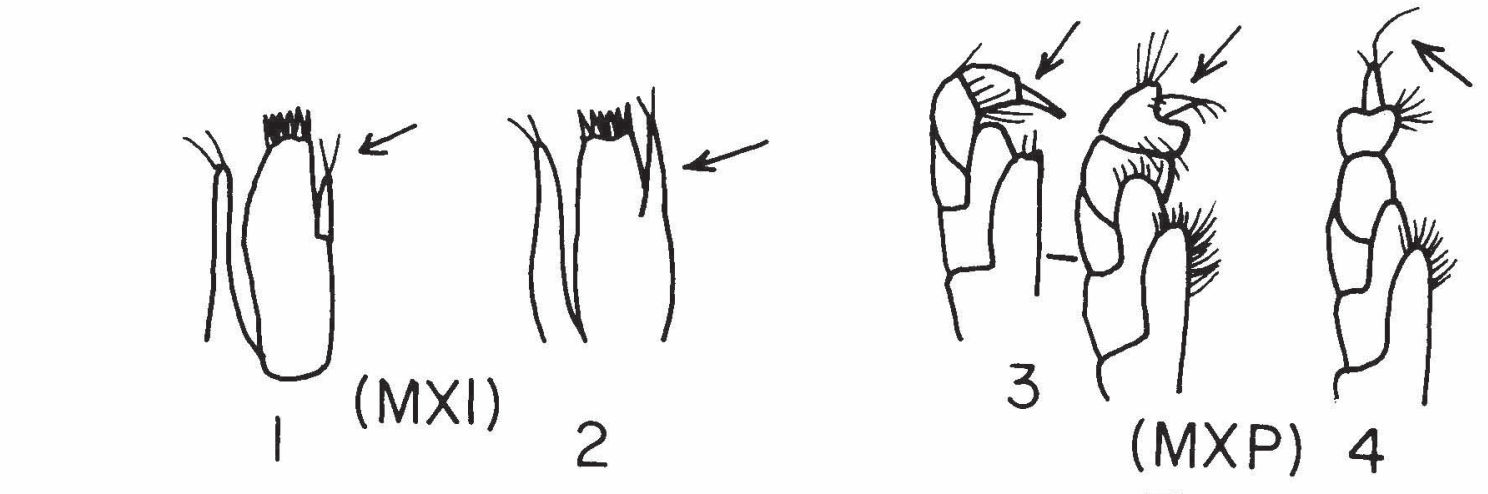

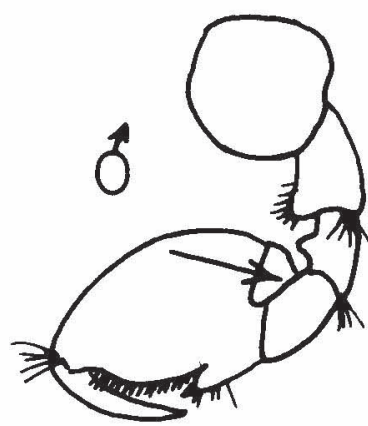

5

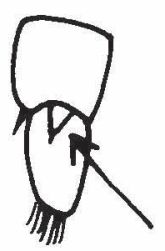

8

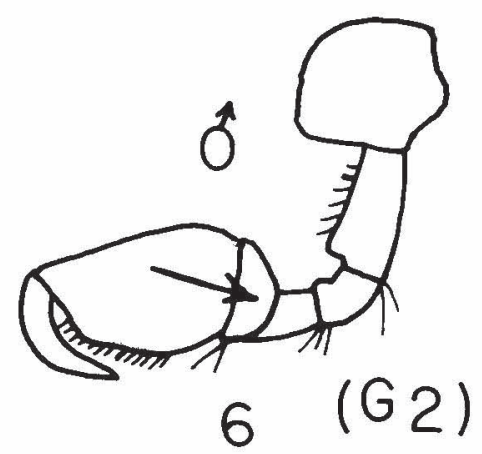

$6\left(G_{2}\right)$

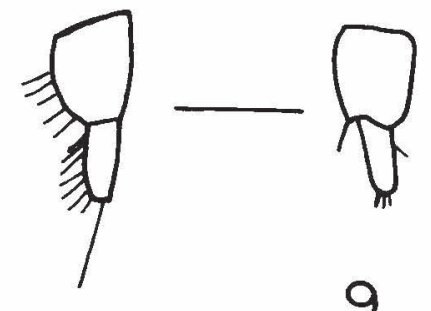

(U3)

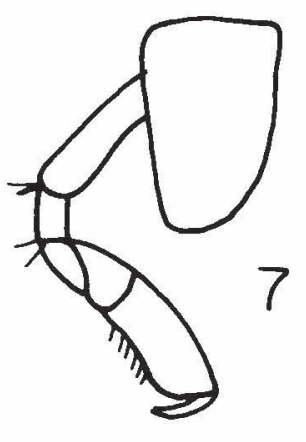

7

Lámina 41.- Familia HYALIDAE (final).

2, 3, 5, $9 \Rightarrow$ Hyale;

1, 4, 7,9 Micropythia;

1, 3, 6, $8 \Rightarrow$ Parhyale.

(ver TALITRIDAE) 


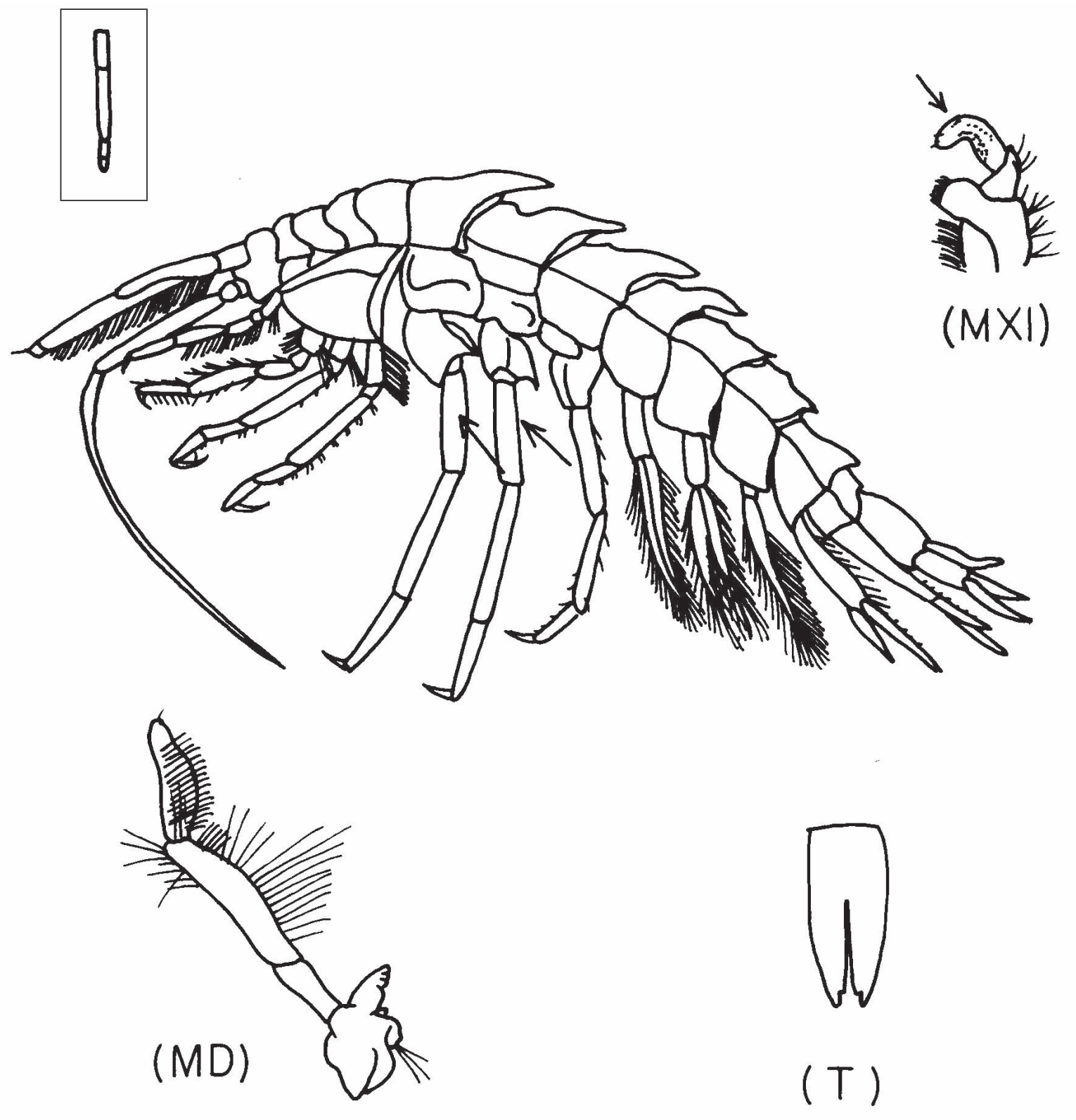

Lámina 42.- Familia HYPERIOPSIDAE (un solo género en el área) $\rightarrow$ Parargissa. 


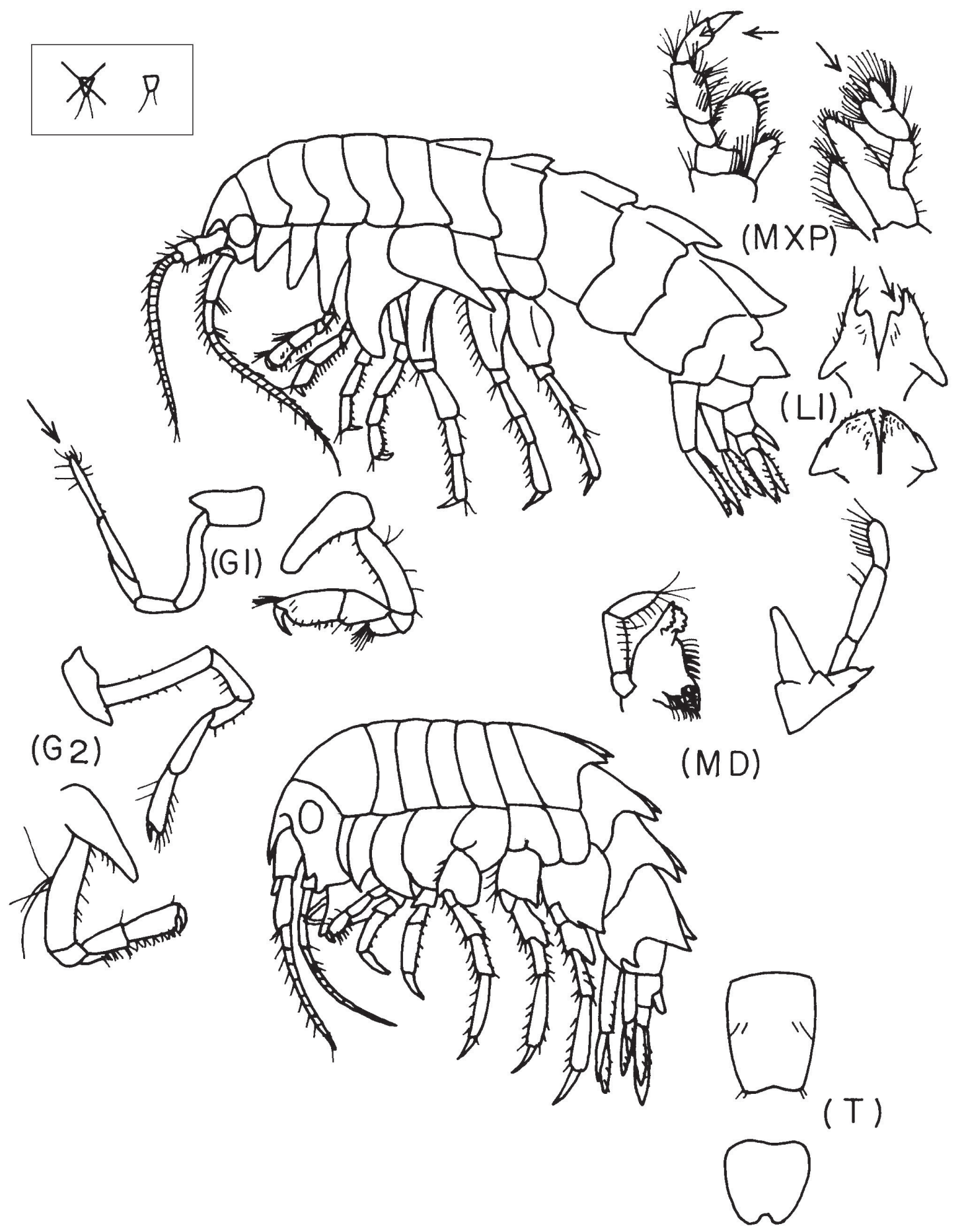

Lámina 43.- Familia IPHIMEDIIDAE (continúa). 
$x_{2}^{8}$

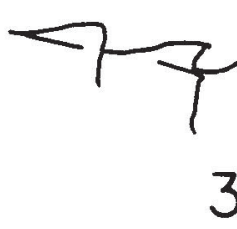

3

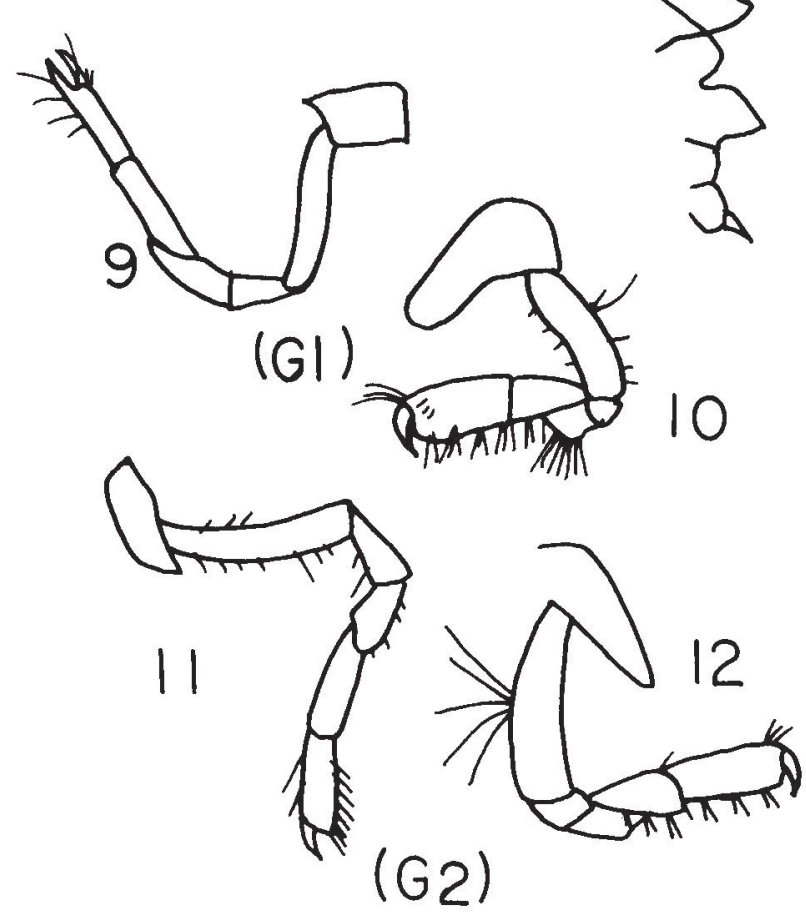

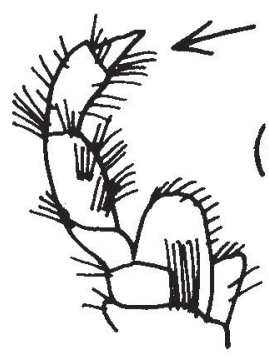

5

$(\mathrm{M} \times \mathrm{P})$

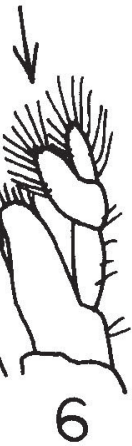

4
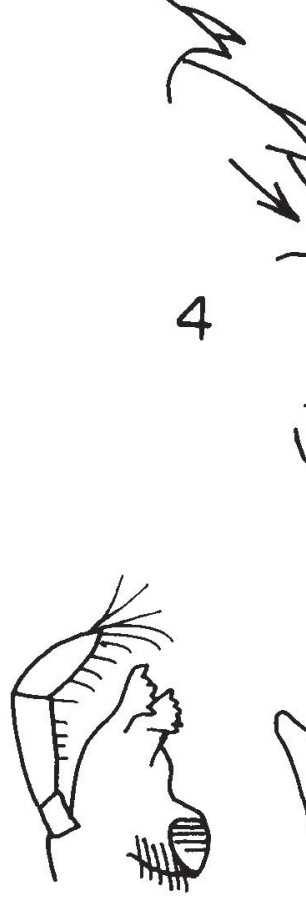

13

14

(MD)

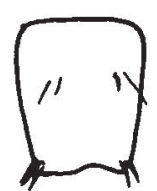

15
$(T)$<smiles>C1CCCCCCCC1</smiles>

16

Lámina 44.- Familia IPHIMEDIIDAE (final).

1, 4, 6, 7, 9, 11, 14, $15 \Rightarrow$ Iphimedia;

2, 3, 5, 8, 10, 12, 13, $16 \Rightarrow$ Epimeria. 

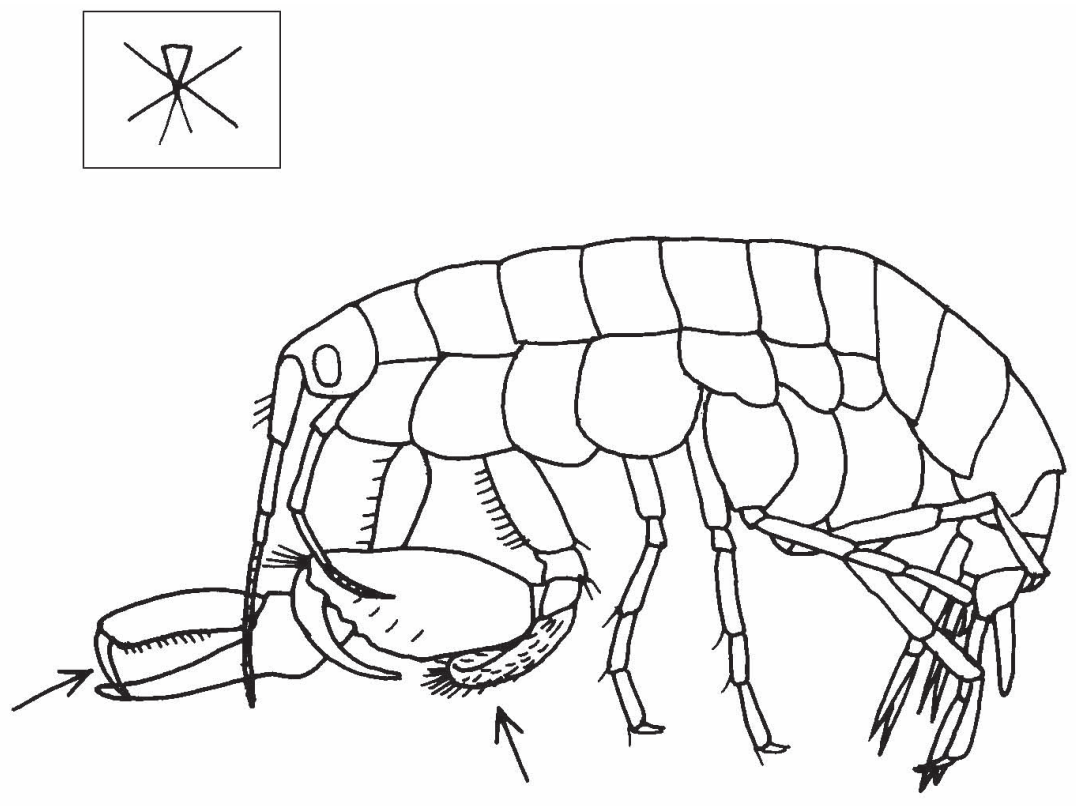

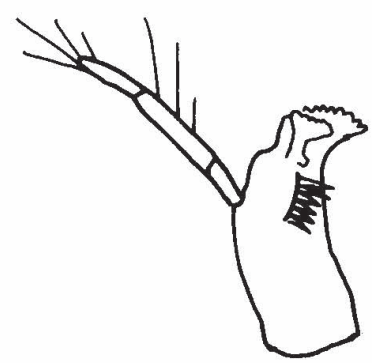

(MD)

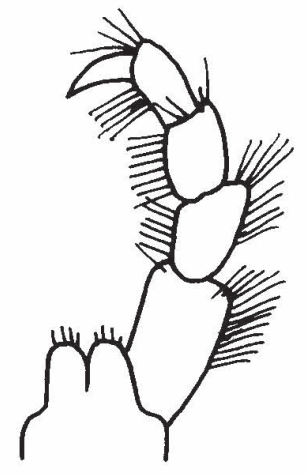

$(\mathrm{MXP})$

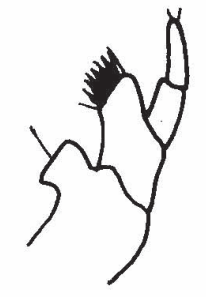

$(M \times 1)$
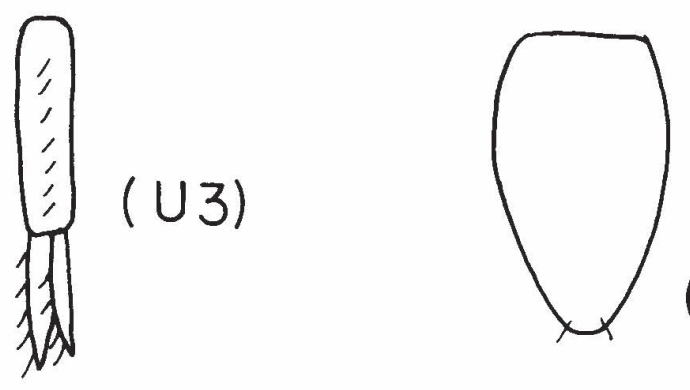

( $\mathrm{T})$

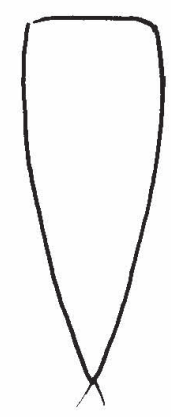

Lámina 45.- Familia LEUCOTHOIDAE (un solo género en el área) $\rightarrow$ Leucothoe. 


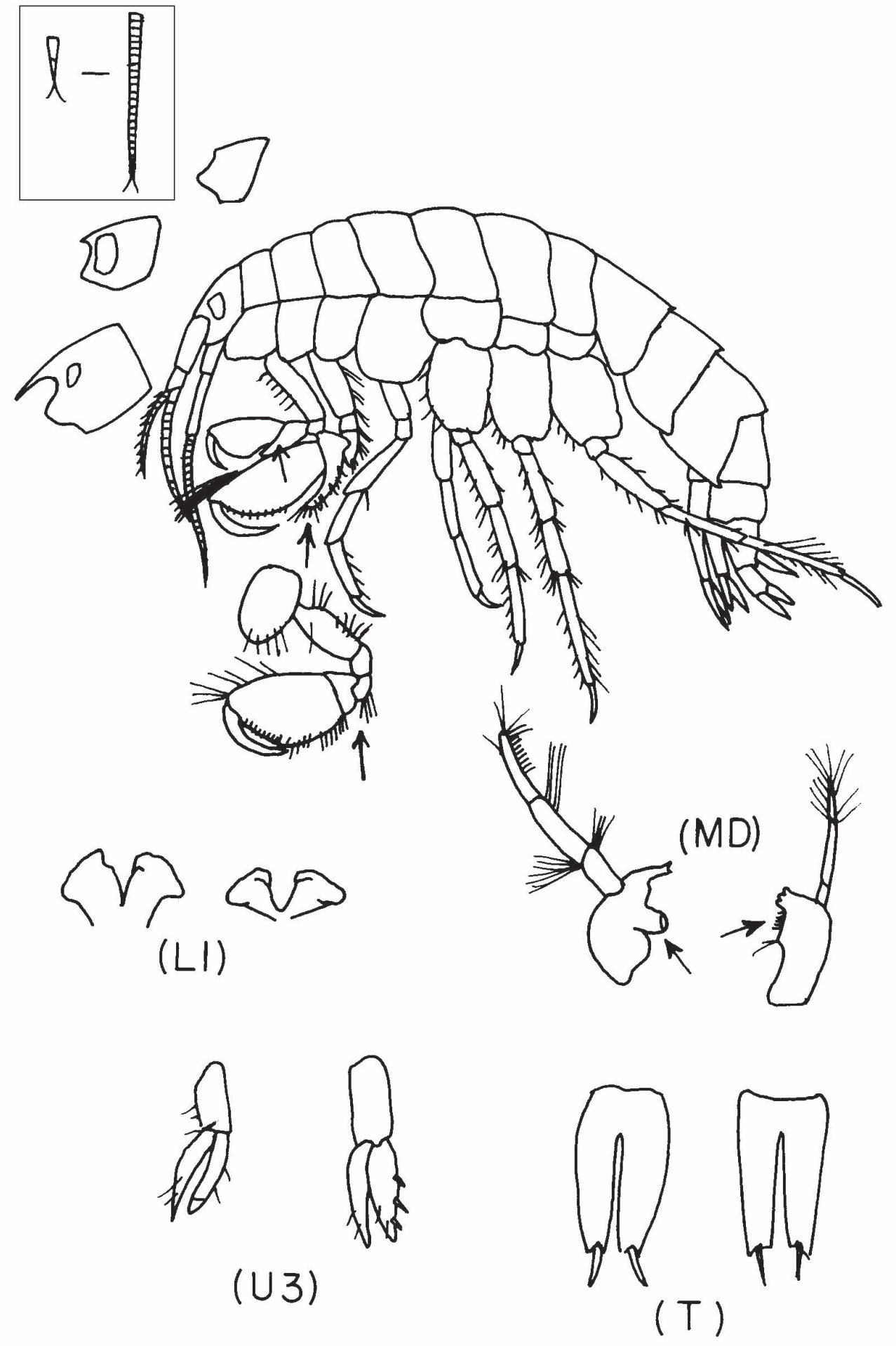

Lámina 46. Familia LILJEBORGIIDAE (continúa). 


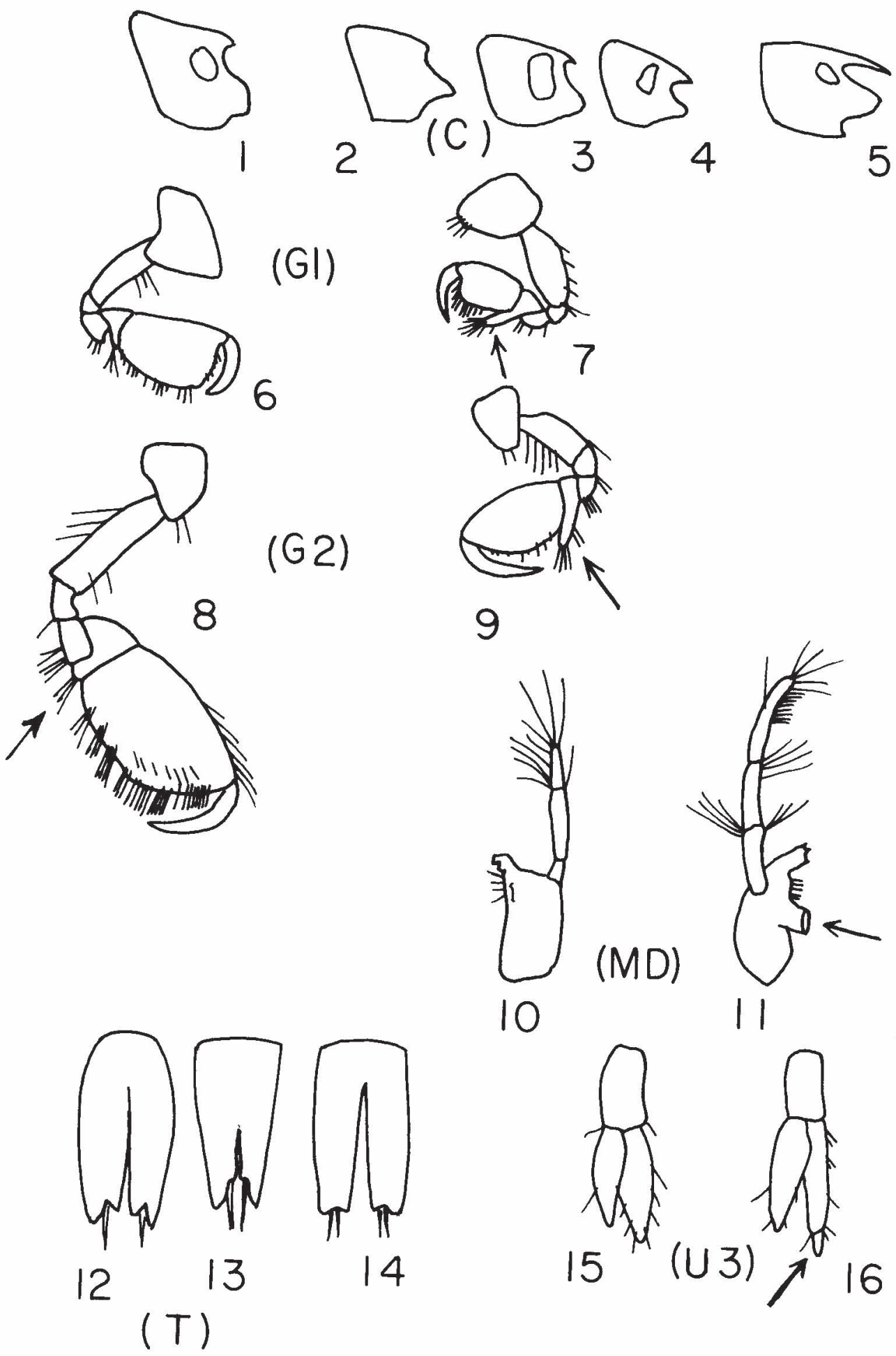

Lámina 47.- Familia LILJEBORGIIDAE (final).

$1,6,8,10,14,16 \Rightarrow$ Listriella;

2-4, 7, 9, 10, 12-14, $15 \Rightarrow$ Liljeborgia;

$5-6,8,11,14,16 \Rightarrow$ Sextonia. 

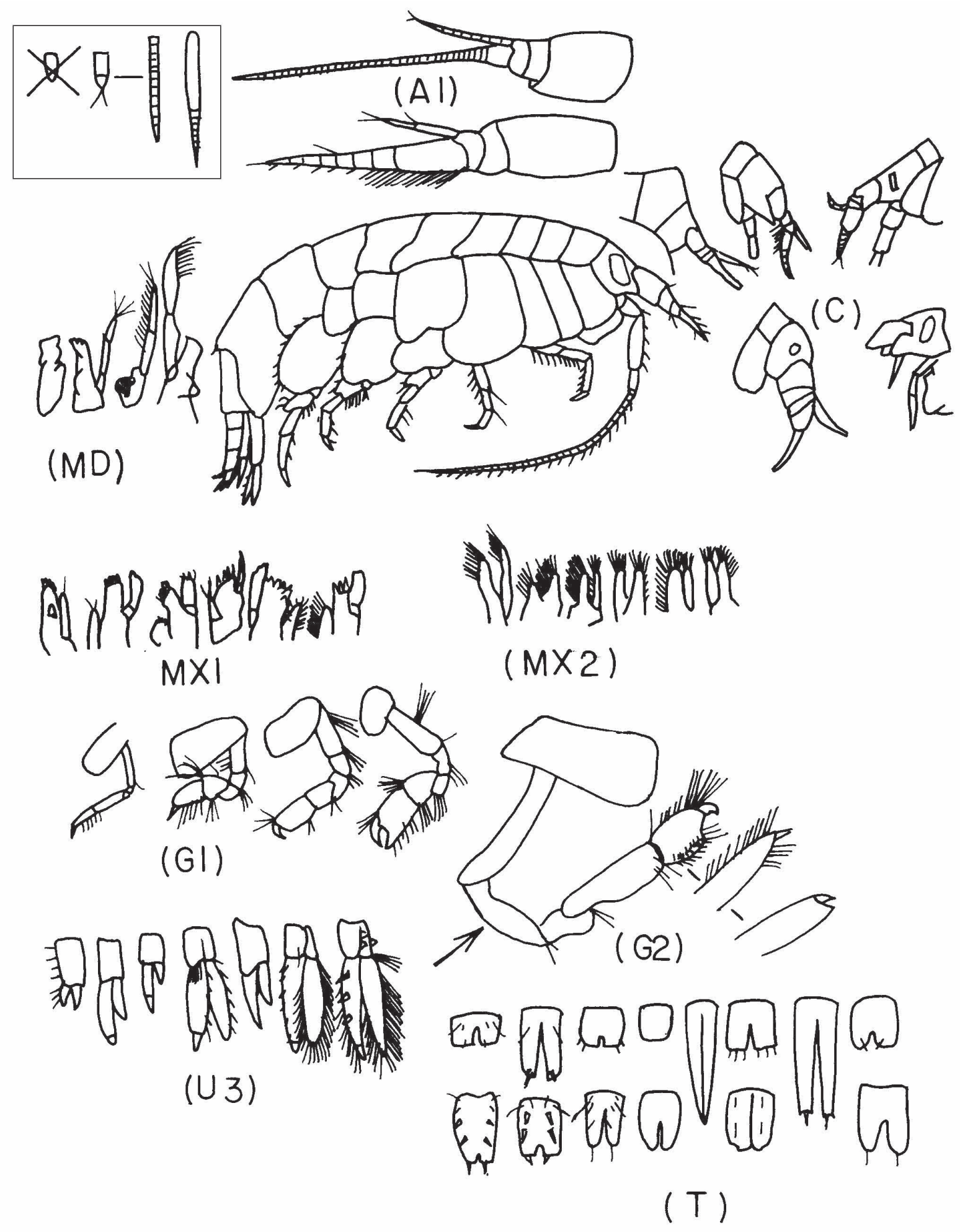

Lámina 48.— Familia LYSIANASSIDAE (continúa). 


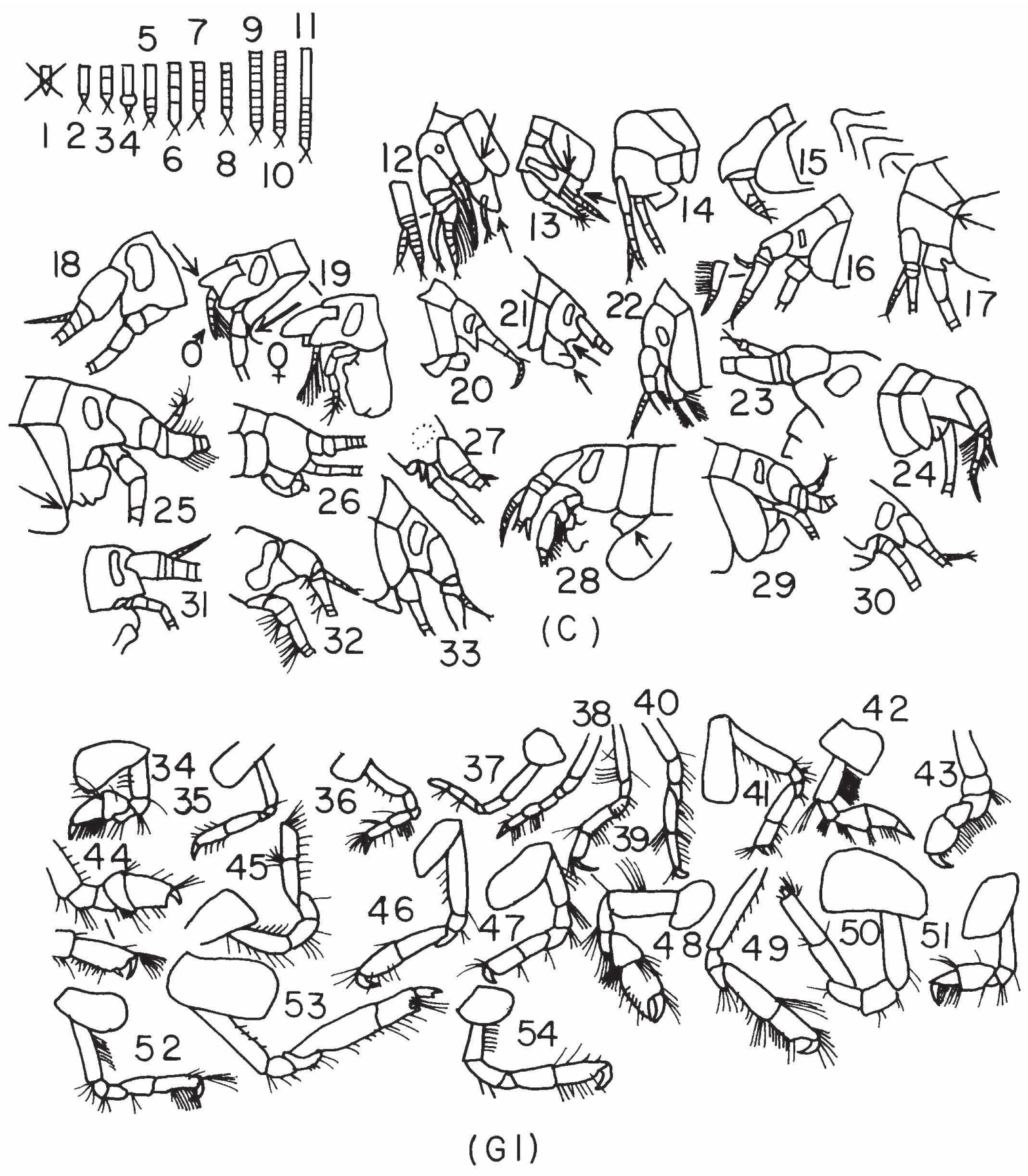

Lámina 49.— Familia LYSIANASSIDAE (continúa). 

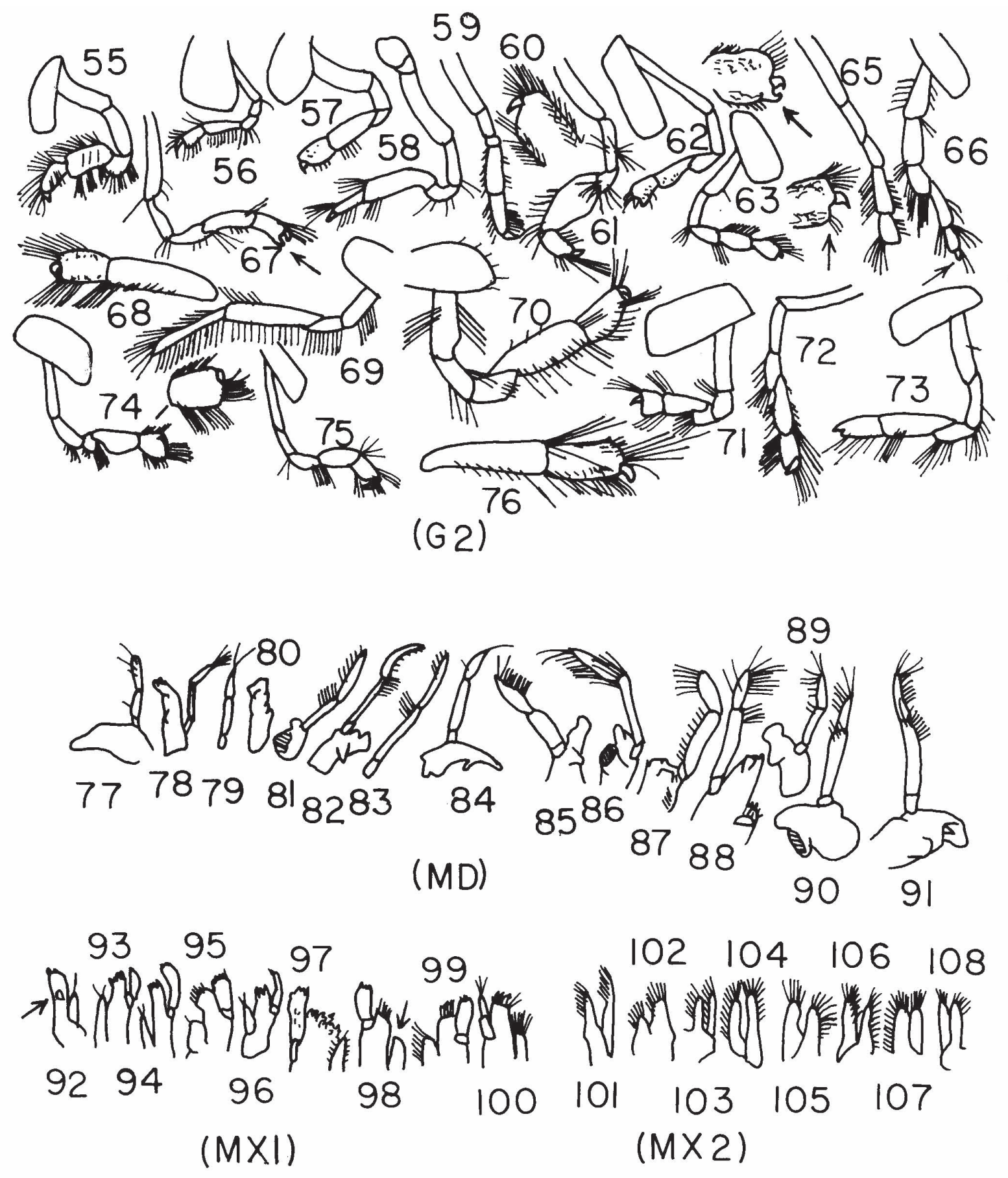

Lámina 50.— Familia LYSIANASSIDAE (continúa). 


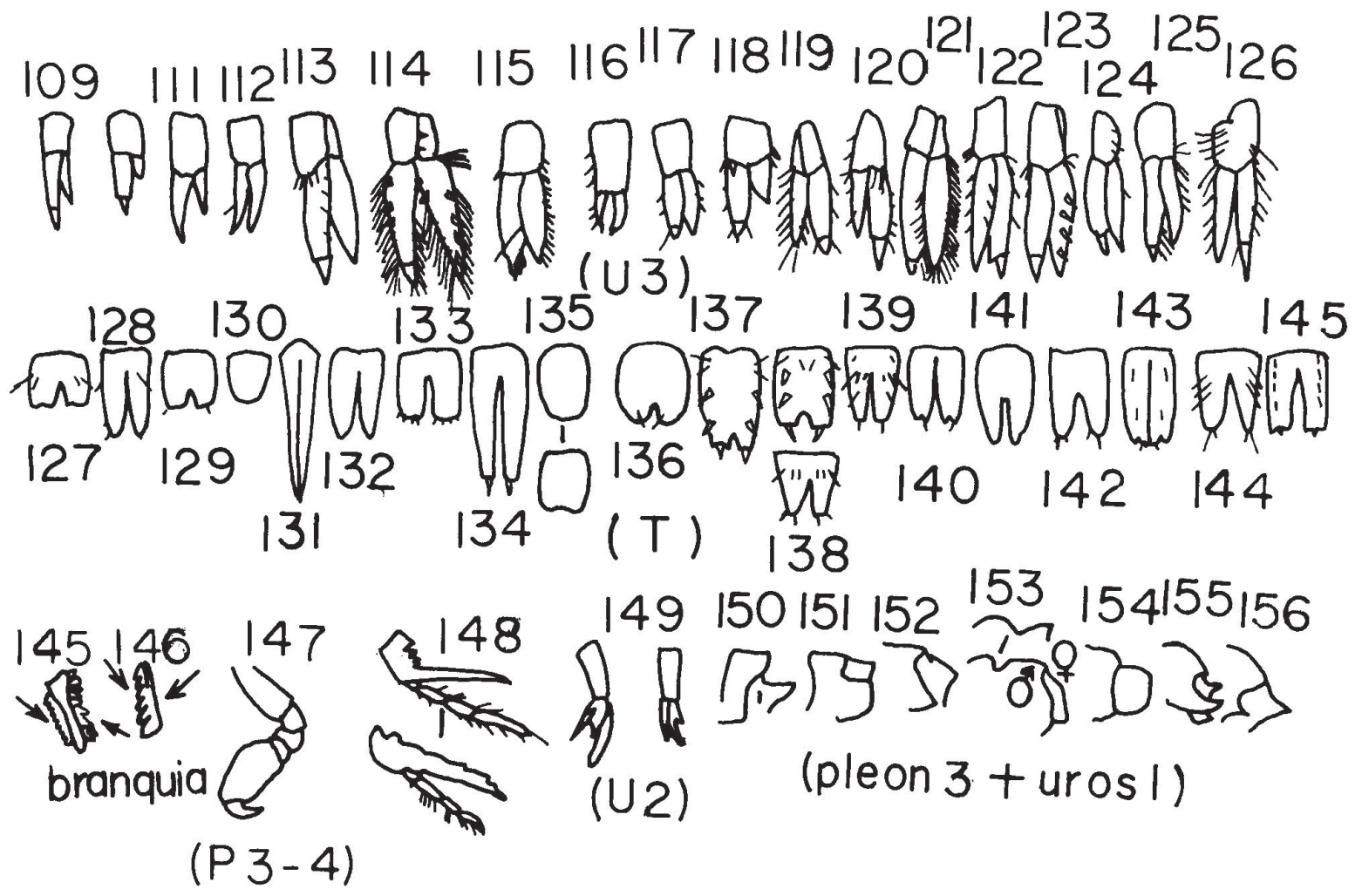

Lámina 51. - Familia LYSIANASSIDAE (final).

6-7, 12, 34, 55, 77, 92, 101, 109, 127 Acidostoma;

7, 13, 35, 56, 78, 93, 110, $128 \Rightarrow$ Ambasia;

7, 14, 36, 57, 79, 94, 111, $129 \Rightarrow$ Bathyamaryllis;

$1,15,37,58,80,95,102,112,130,147 \Rightarrow$ Crybelocephalus;

4, 17, 38, 59, 131, $148 \Rightarrow$ Cyphocaris;

2-3, 16, 39, 60, 81, 113, 132, $146 \Rightarrow$ Hippomedon;

10, 18, 40, 61, 82, 96, 103, 114, 133, 145, $149 \Rightarrow$ Ichnopus;

1, 19, 41, 62, 83, 97, 115, 134, 146, $150 \Rightarrow$ Lepidepecrum;

3-8, 20, 42, 63, 84, 98, 116, 135, $151 \rightarrow$ Lysianassa;

$3-8,21,42,64,84,98,116,135,151 \Rightarrow$ Lysianassina;

$8,22,43,65,94,101,117,136,148,152 \Rightarrow$ Onisimus;

4, 23, 44, 67, 94, 104, 118, 137, 146, $153 \rightarrow$ Orchomene;

3-6, 25, 45, 66, 85, 99, 119, 128, $154 \Rightarrow$ Paracallisoma;

6, 24, 46, 68, 86, 120, 138, $155 \Rightarrow$ Paracentromedon;

26, 47, 69, 87, 99, 121, $139 \Rightarrow$ Paralicella;

7, 27, 48, 70, 88, 100, 105, 122, $128 \Rightarrow$ Podoprion;

$11,28,49,72,93,106,123,144 \Rightarrow$ Schisturella;

5, 29, 50, 73, 99, 107, 124, 128, $146 \rightarrow$ Scopelocheirus;

2-6, 30, 51, 71, 89, 108, 109, 140, 146 Socarnes;

5, 31, 52, 74, 93, 109, 141, $145 \Rightarrow$ Socarnopsis;

$8,32,54,75,90,94,108,125,142,152 \rightarrow$ Tmetonyx;

7, 33, 53, 76, 91, 93, 126, 143, $156 \Rightarrow$ Tryphosites. 

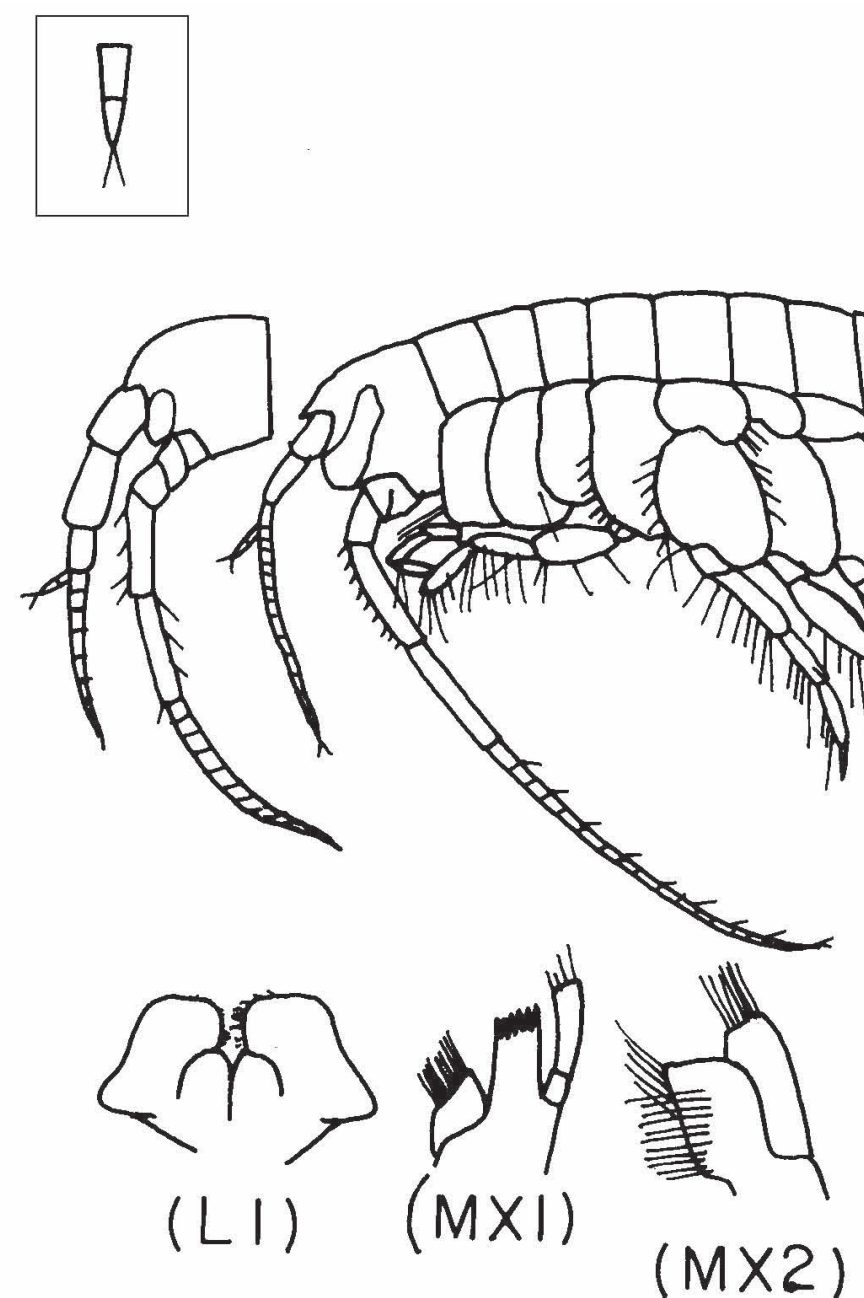

$(M \times 2)$
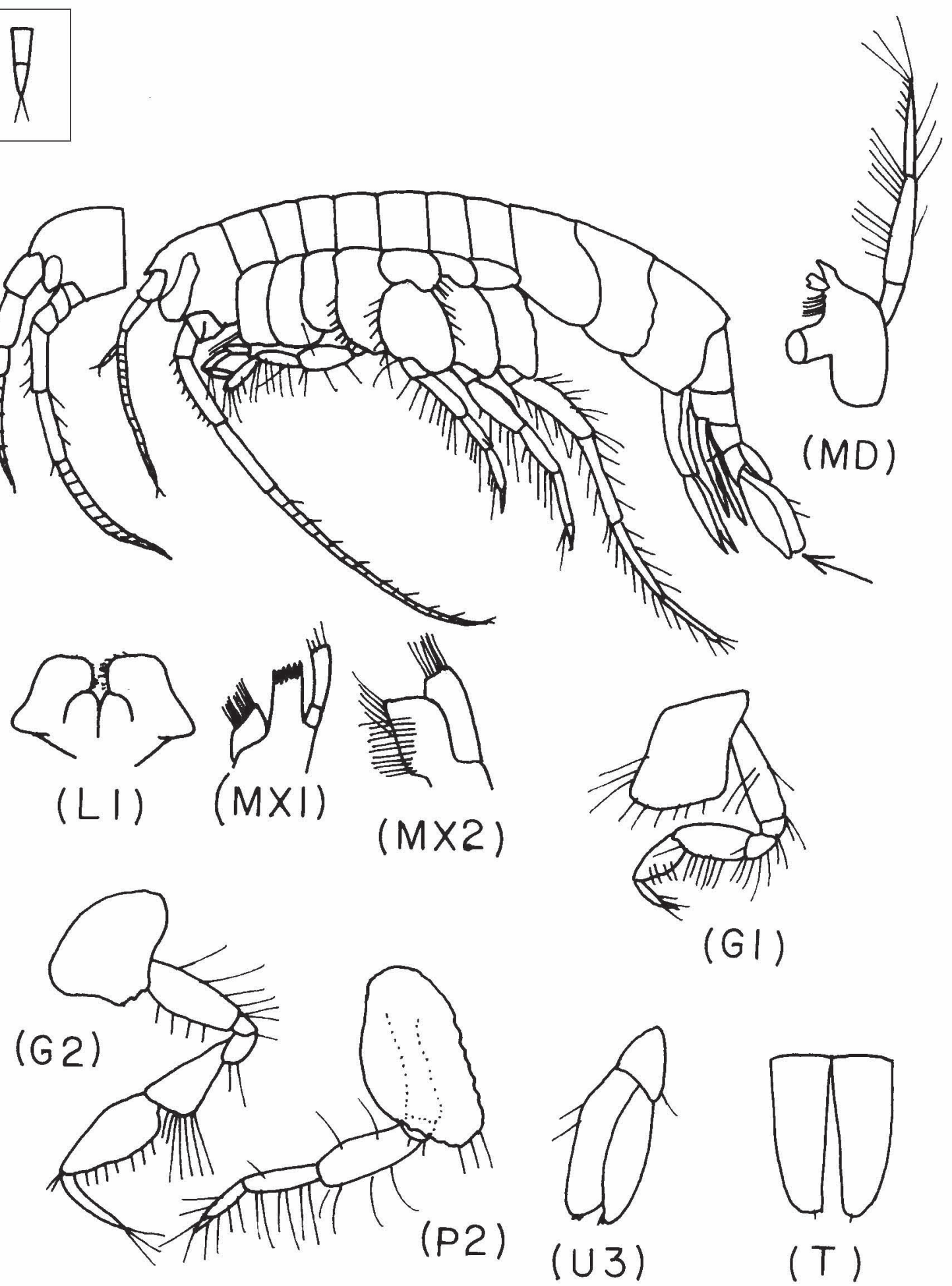

(MD)

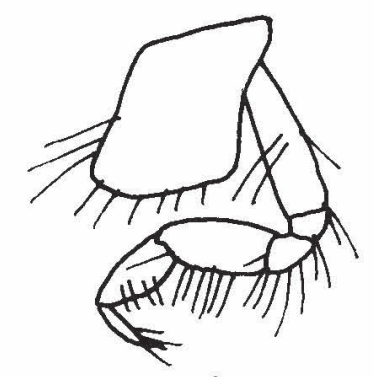

(GI)

Lámina 52.- Familia MEGALUROPIDAE (un solo género en el área) Megaluropus. 

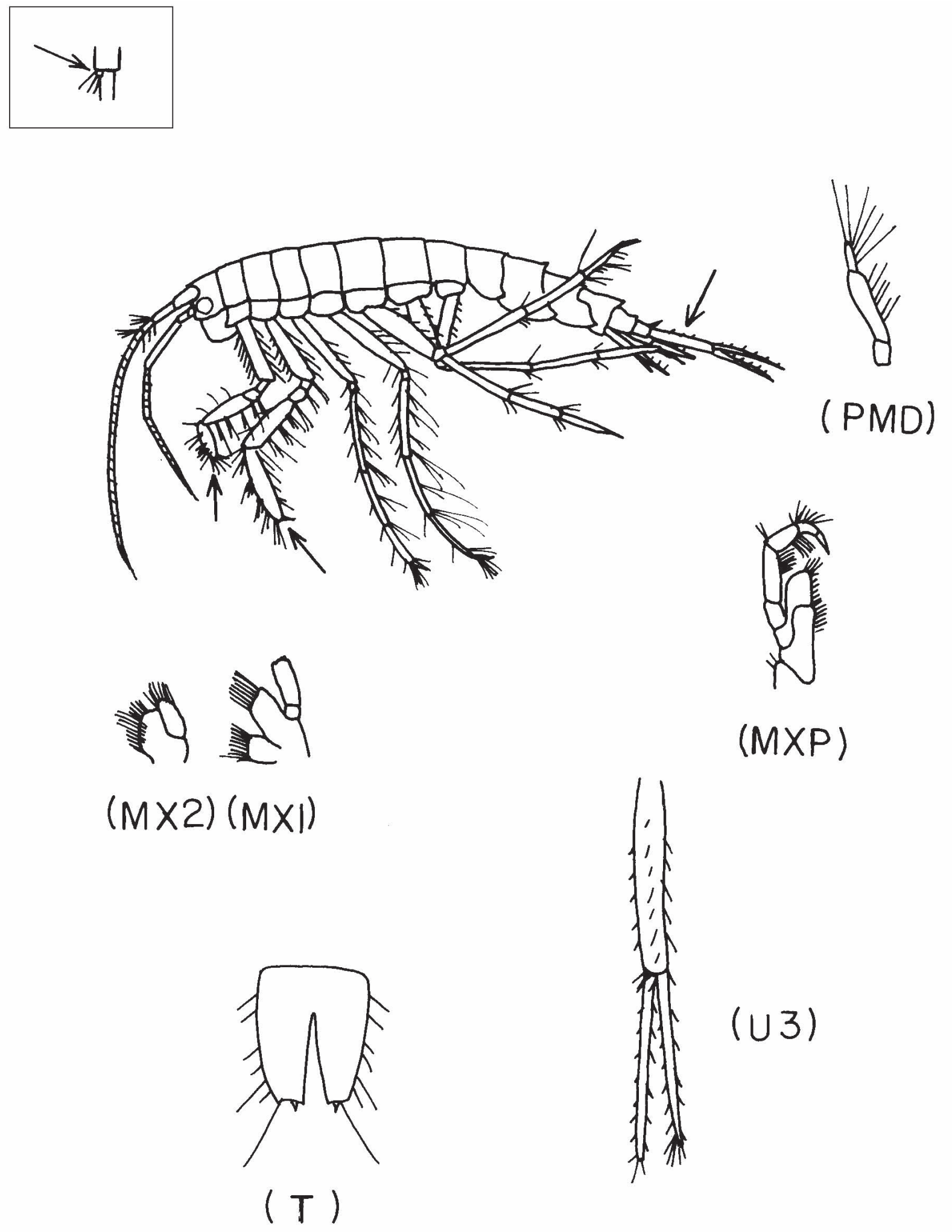

Lámina 53.- Familia MELPHIDIPPIDAE (un solo género en el área) $\Rightarrow$ Melphidippella. 

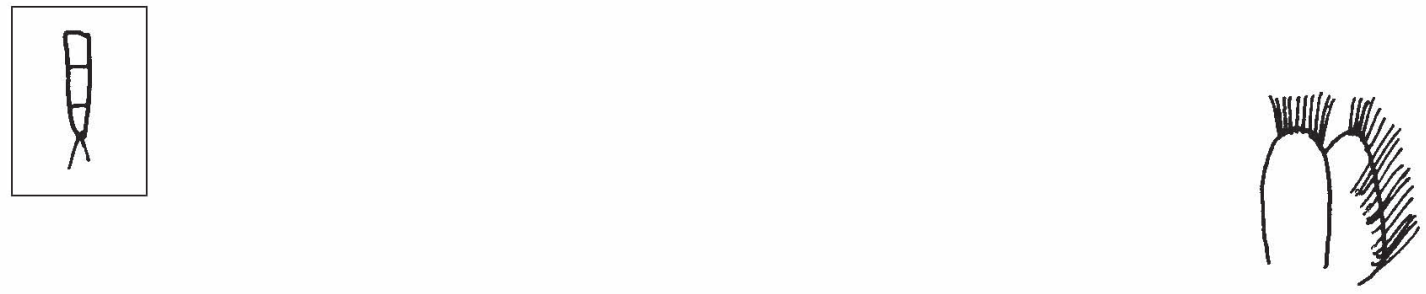

$(\mathrm{M} \times 2)$

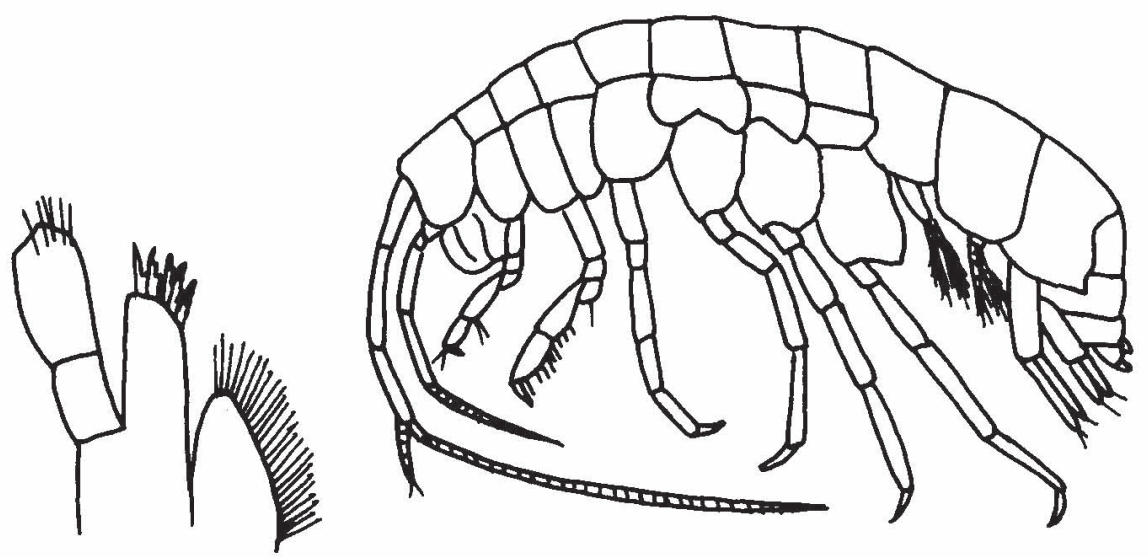

$(M X I)$

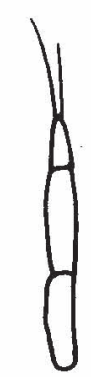

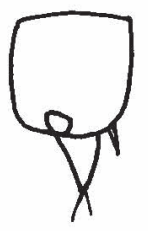

(U3)

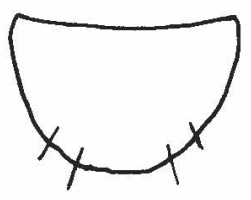

( $\mathrm{T}$ )
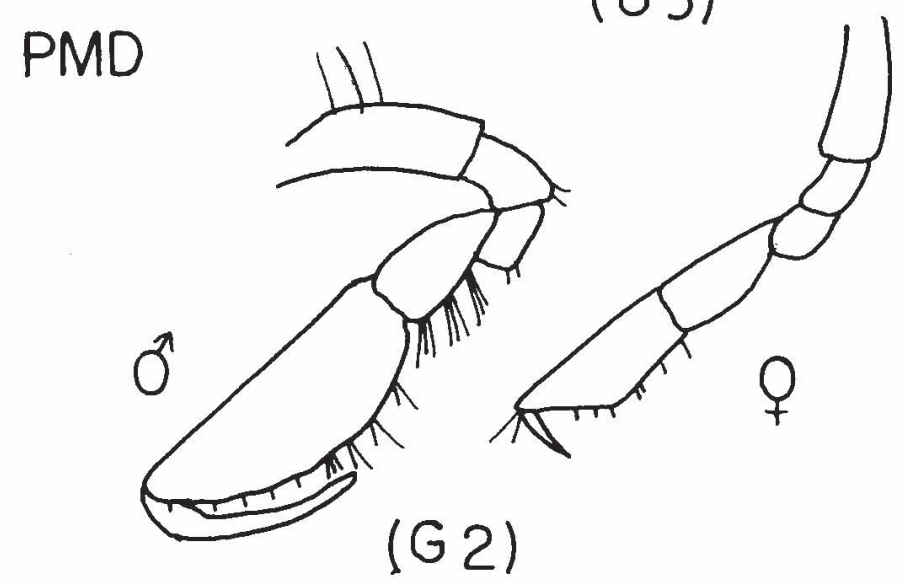

Lámina 54.- Familia METACRANGONYCTIDAE (un solo género en el área) $\$$ Metacrangonyx. 

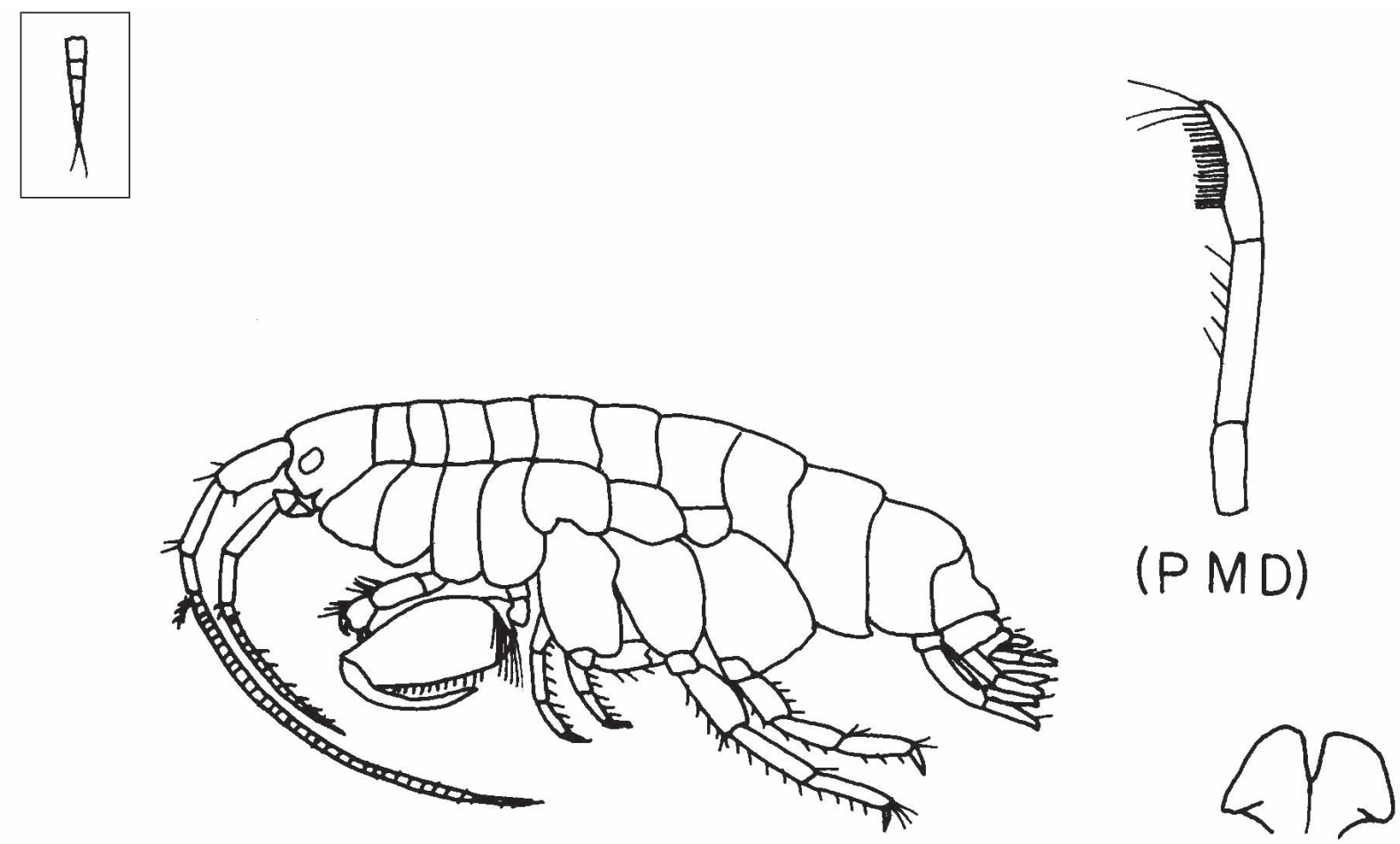

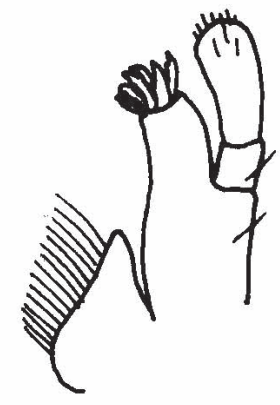

$(M \times 1) \quad(M \times 2)$
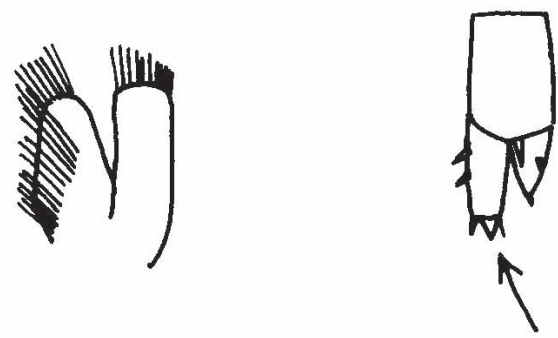

(U3)

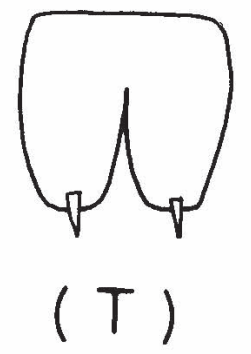

Lámina 55.—Familia NUUANIDAE (un solo género en el área) $\$$ Gammarella. 

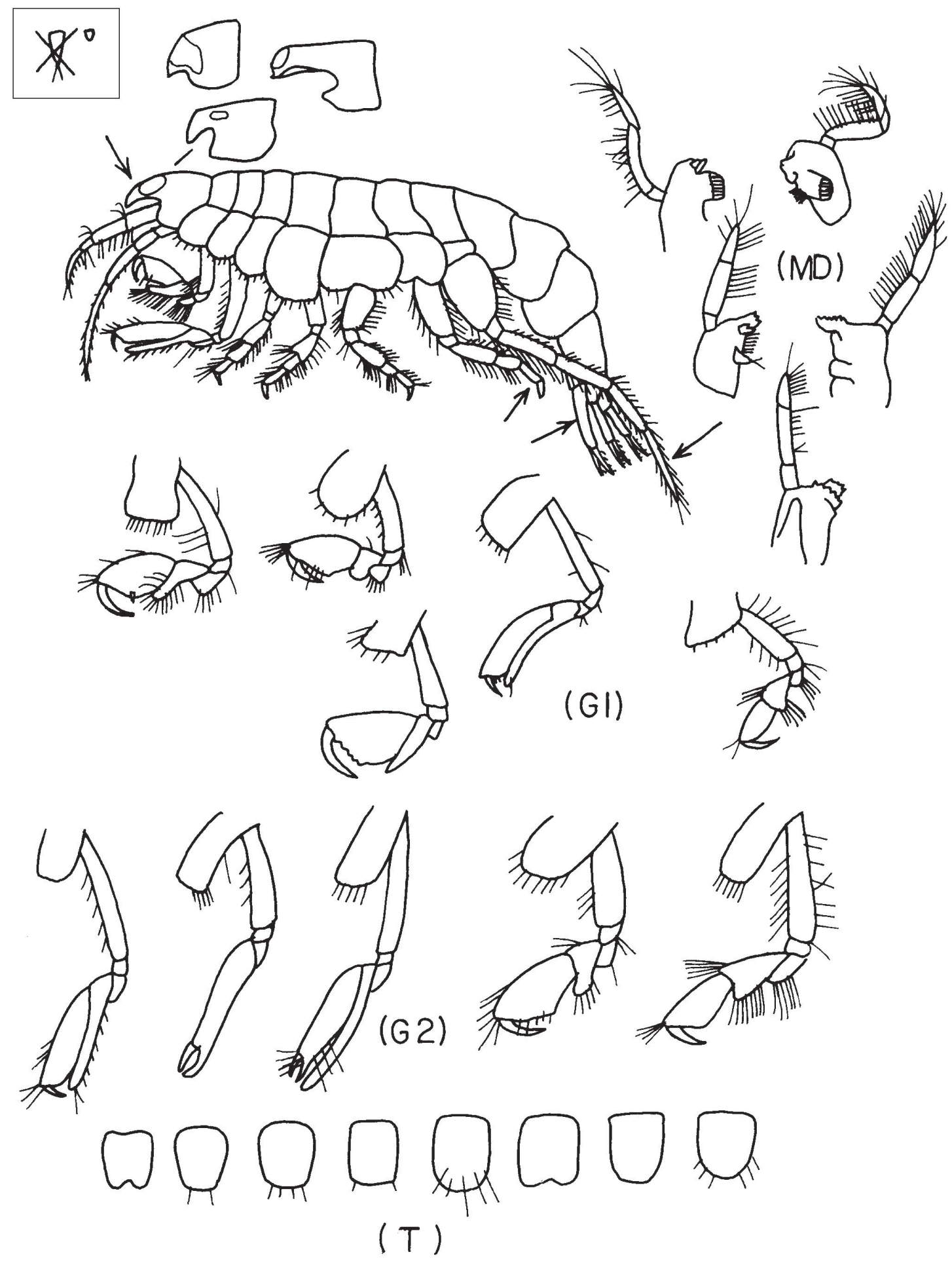

Lámina 56.- Familia OEDICEROTIDAE (continúa). 


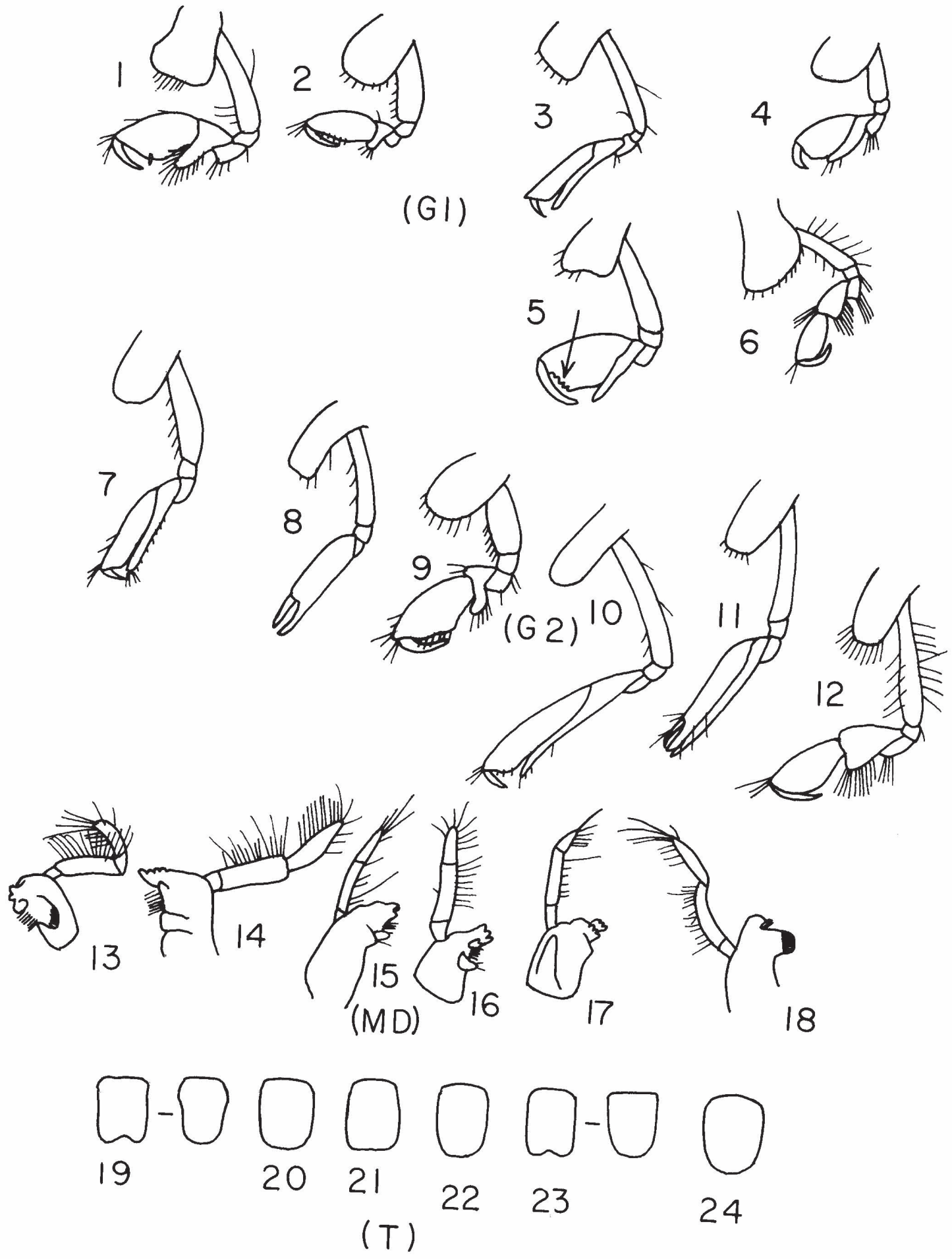

Lámina 57. - Familia OEDICEROTIDAE (final).

1, 7, 13, $19 \Rightarrow$ Monoculodes;

2, 9, 14, $20 \Rightarrow$ Oediceros;

3, 10, 15, $21 \Rightarrow$ Perioculodes;

4, 11, 16, $22 \Rightarrow$ Pontocrates;

$5,8,17,23 \Rightarrow$ Synchelidium. 

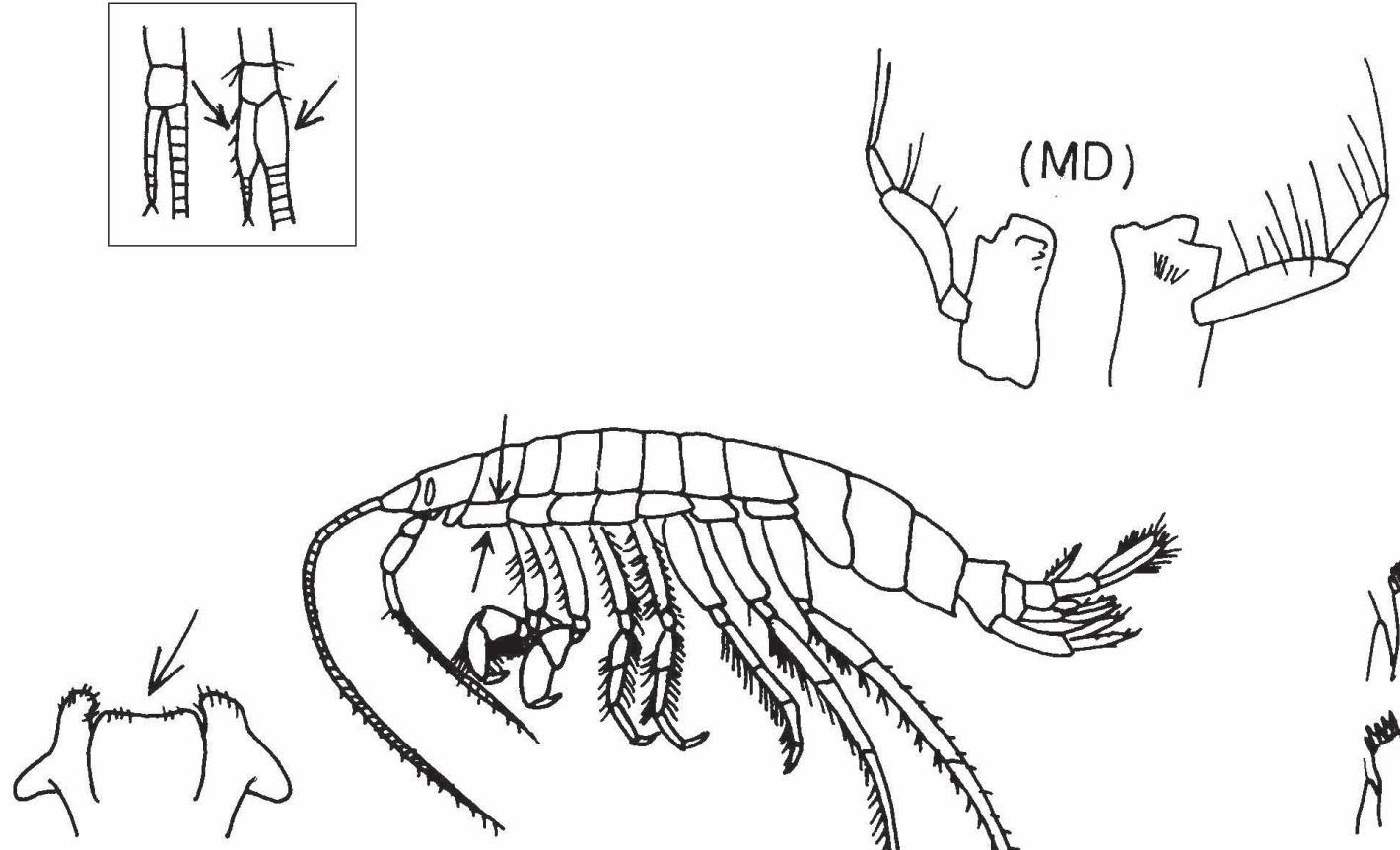

LI
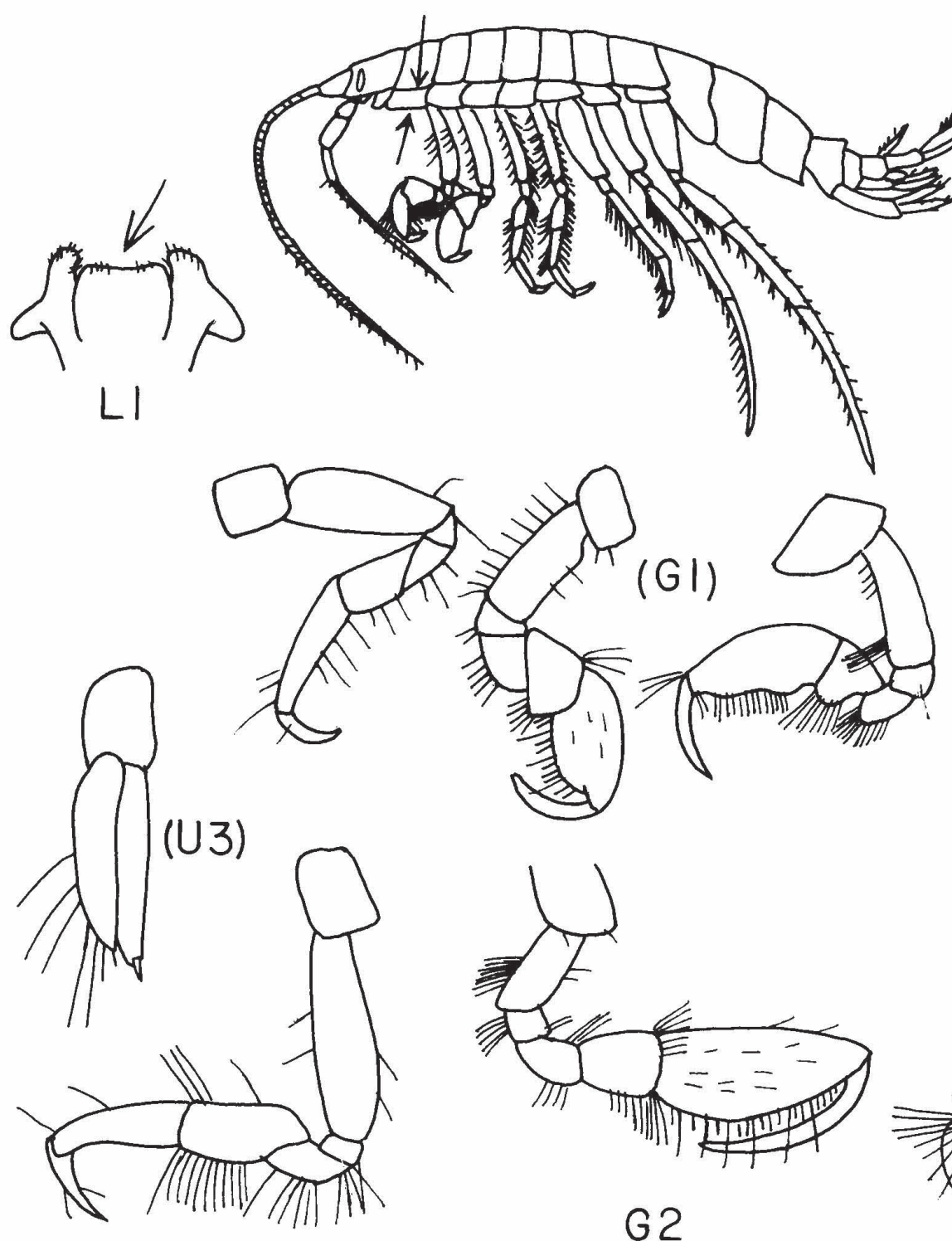

G2

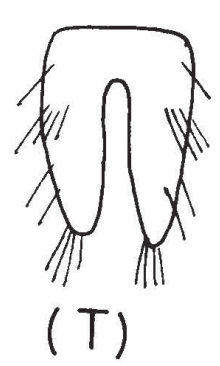

$(\mathrm{M} \times 1)$

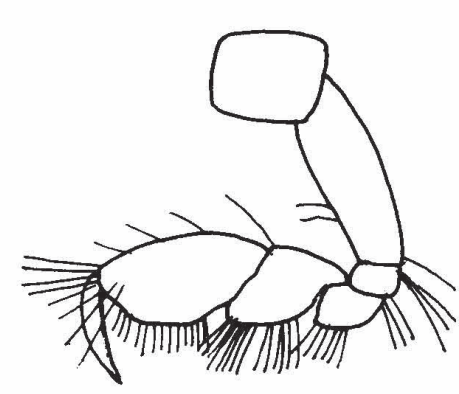

Lámina 58.— Familia PARDALISCIDAE (continúa). 

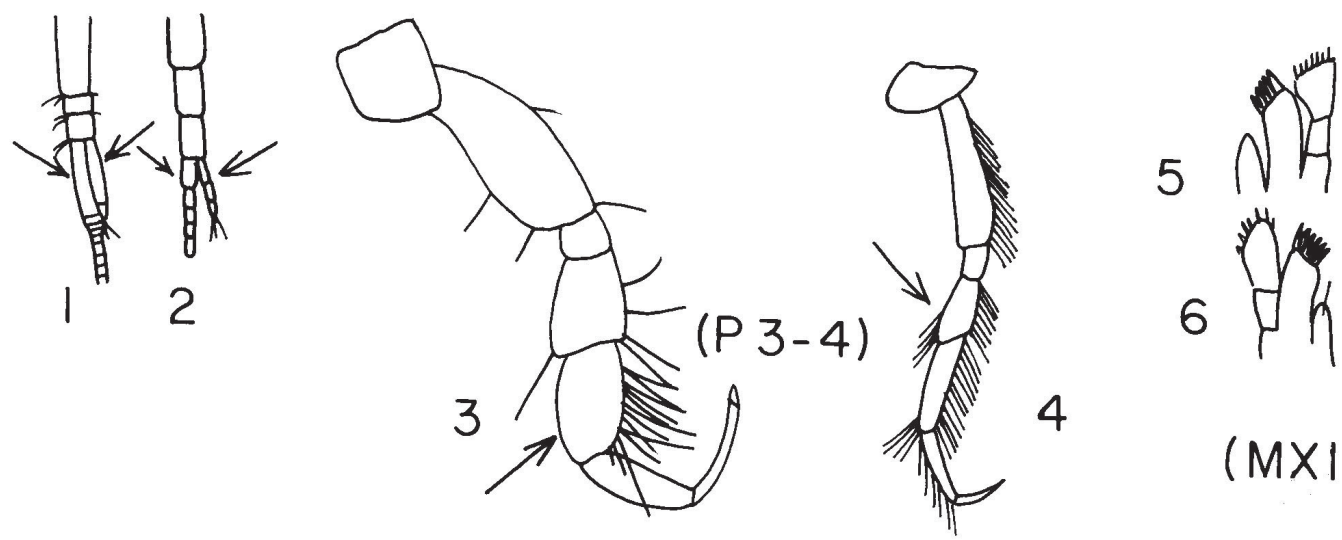

$(M \times 1)$

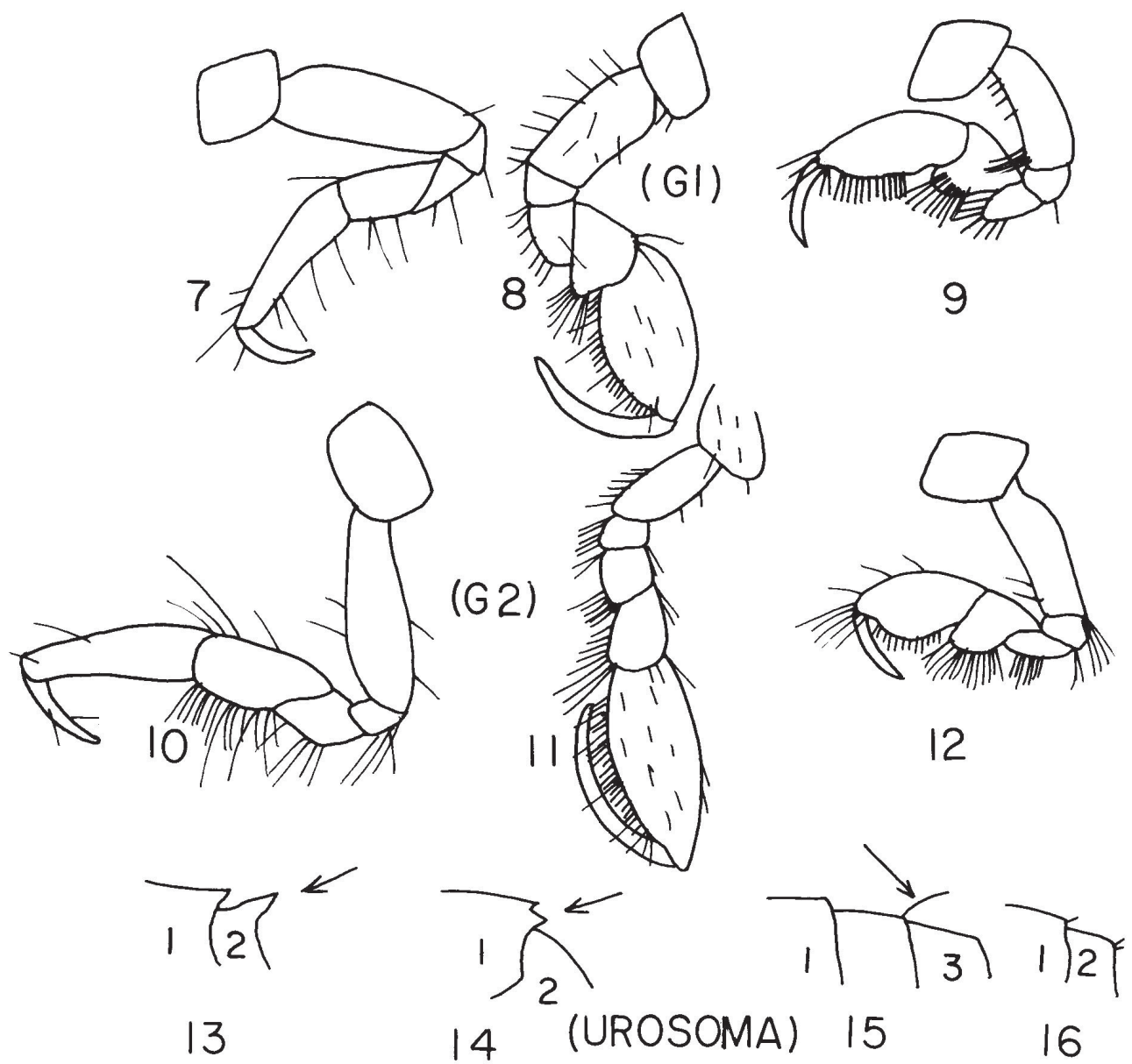

Lámina 59.- Familia PARDALISCIDAE (final).

1, 3, 6, 7, 10, $13 \Rightarrow$ Halice;

1, 3, 6, 7, 10, $15 \Rightarrow$ Halicoides:

1, 4, 5, 9, 12, $14 \Rightarrow$ Nicippe;

2, 4, 6, 8, 11, $16 \rightarrow$ Spelaeonicippe. 

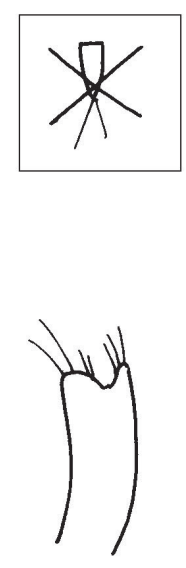

$(M \times 2)$
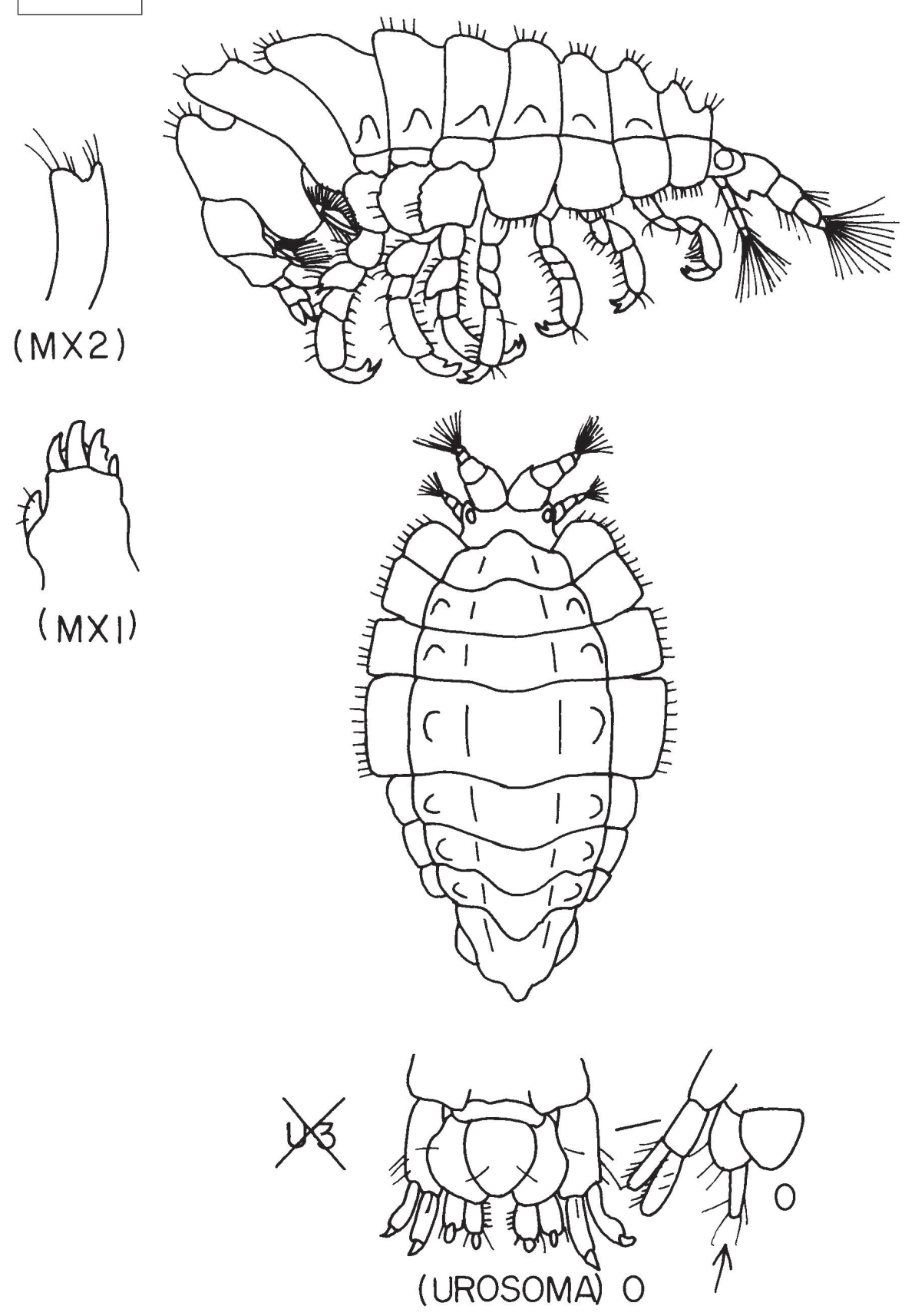

Lámina 60.- Familia PHLIANTIDAE ( un solo género en el área) $\$$ Pereionotus. 


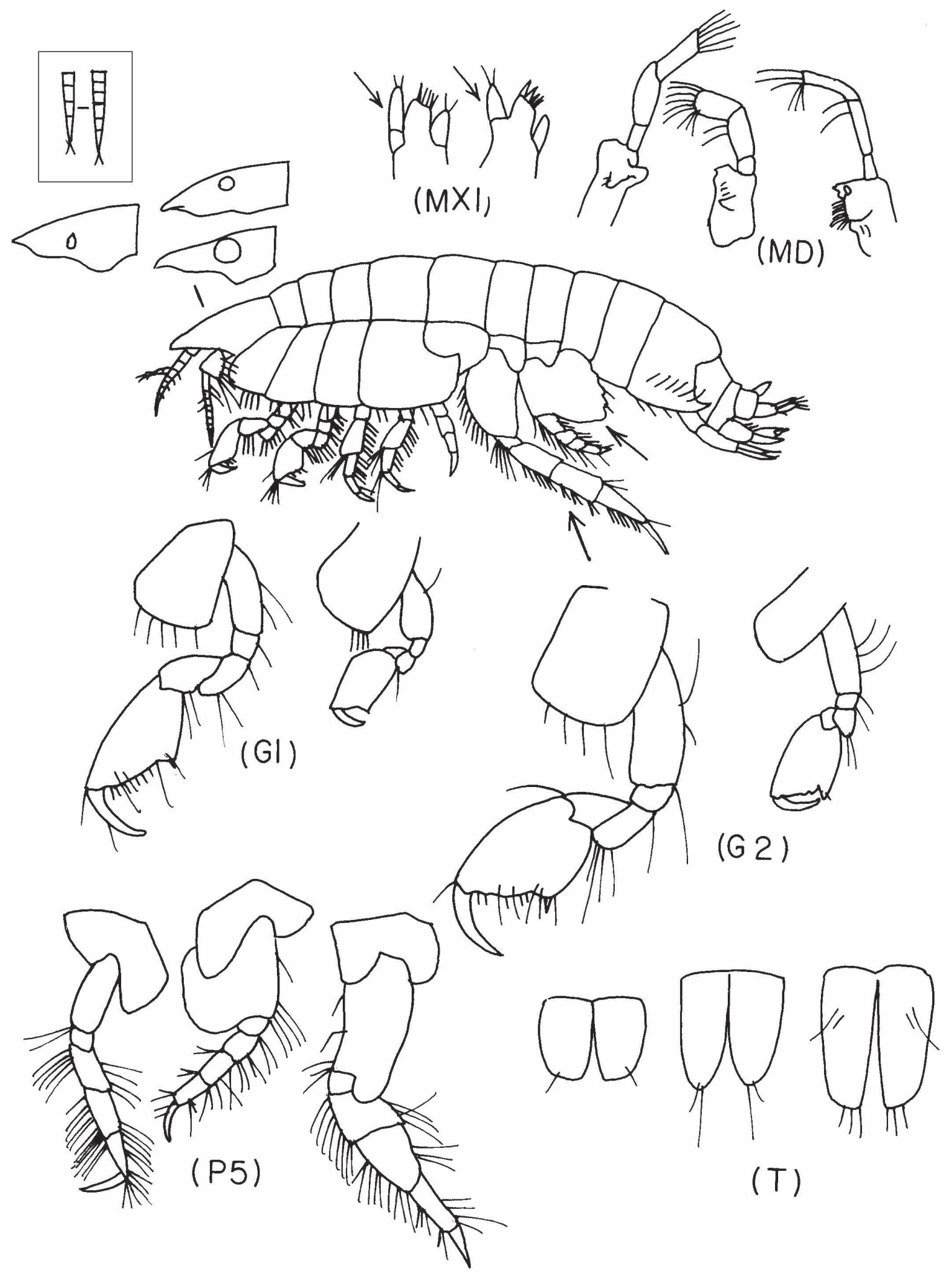

Lámina 61.- Familia PHOXOCEPHALIDAE (continúa). 

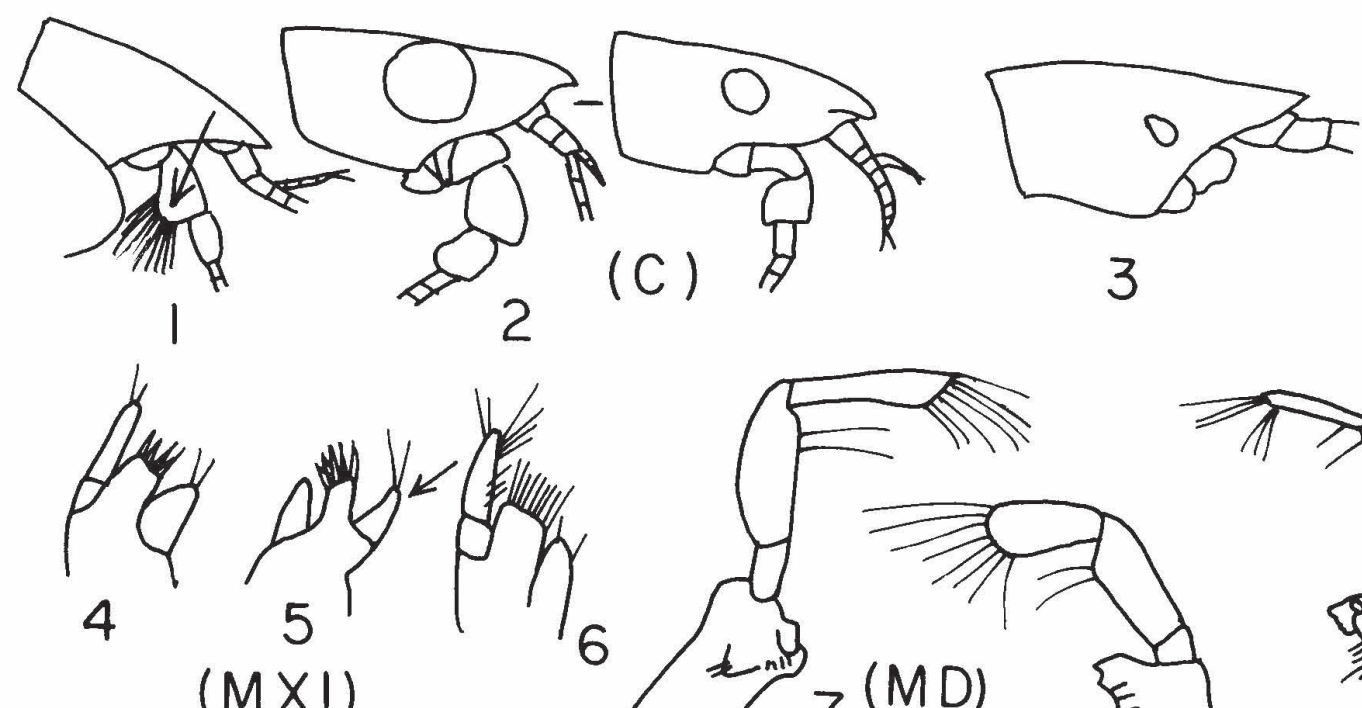

$(\mathrm{M} \times \mathrm{I})$
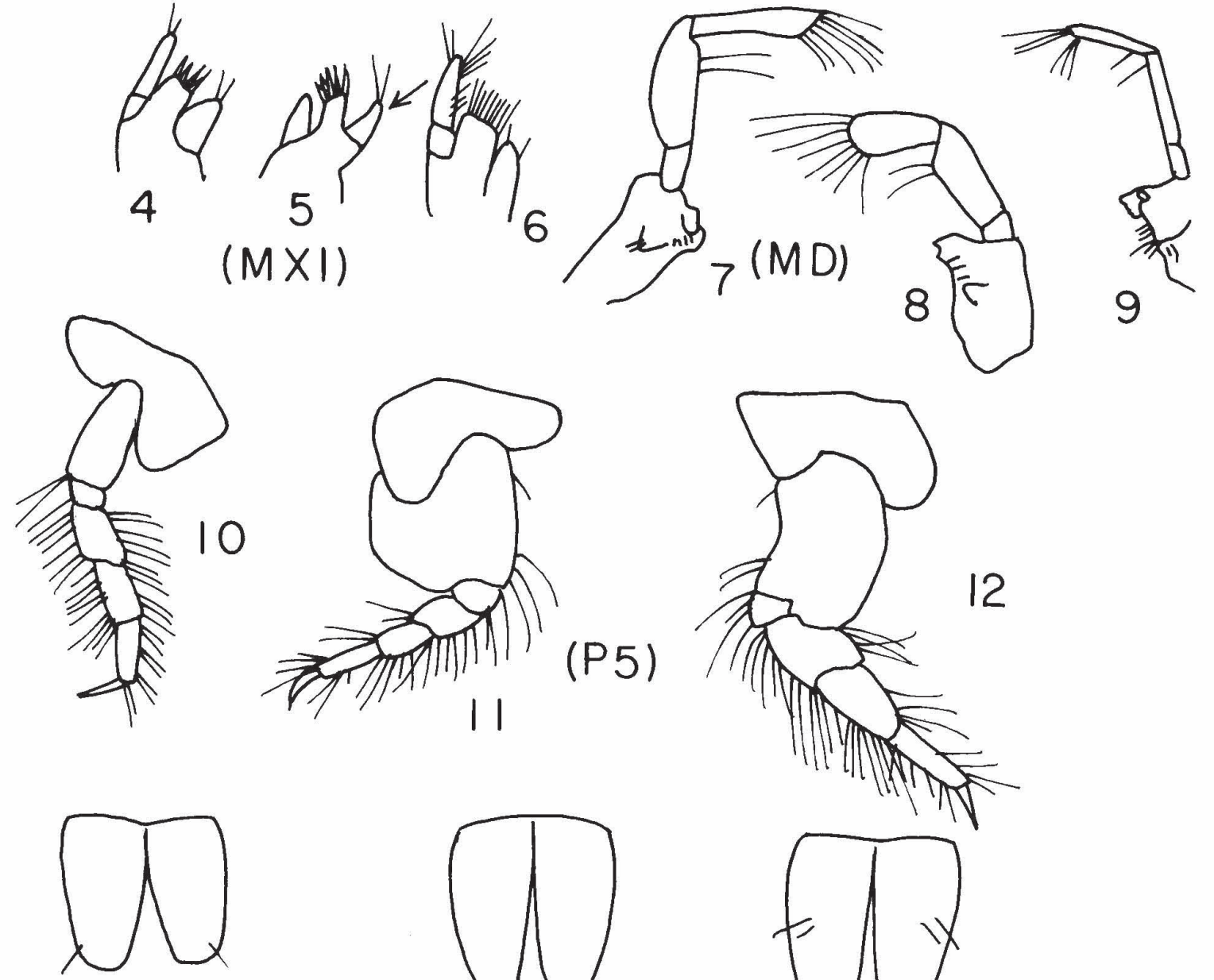

13
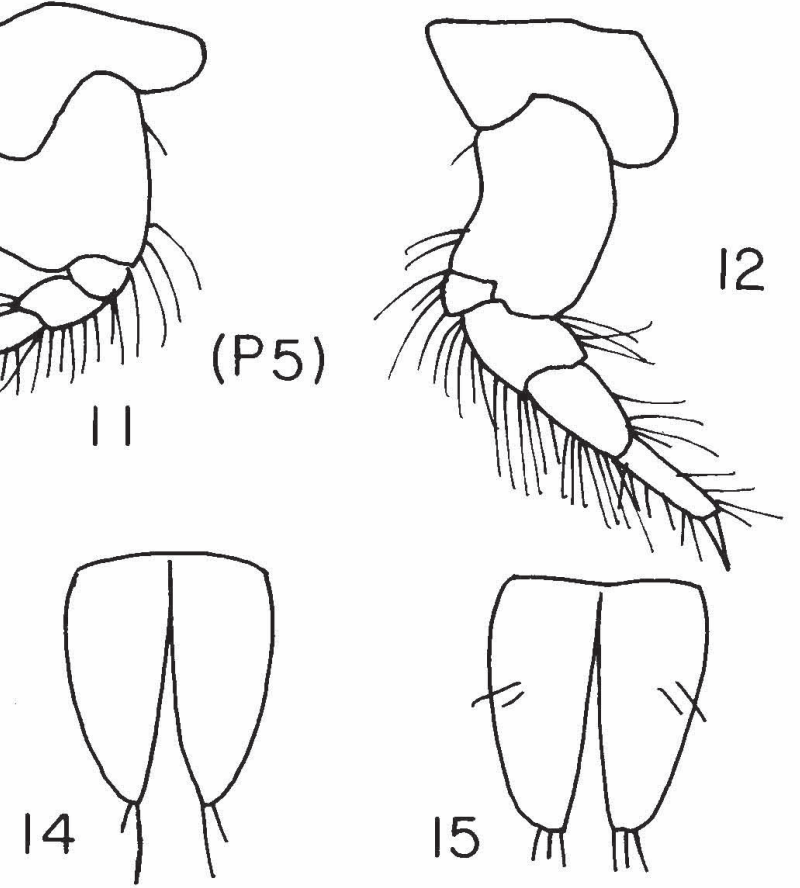

( T )

Lámina 62.- Familia PHOXOCEPHALIDAE (final).

1, 4, 7, 10, $13 \rightarrow$ Harpinia;

2, 5, 8, 11, $14 \Rightarrow$ Metaphoxus;

3, 6, 9, 12, $15 \Rightarrow$ Paraphoxus. 


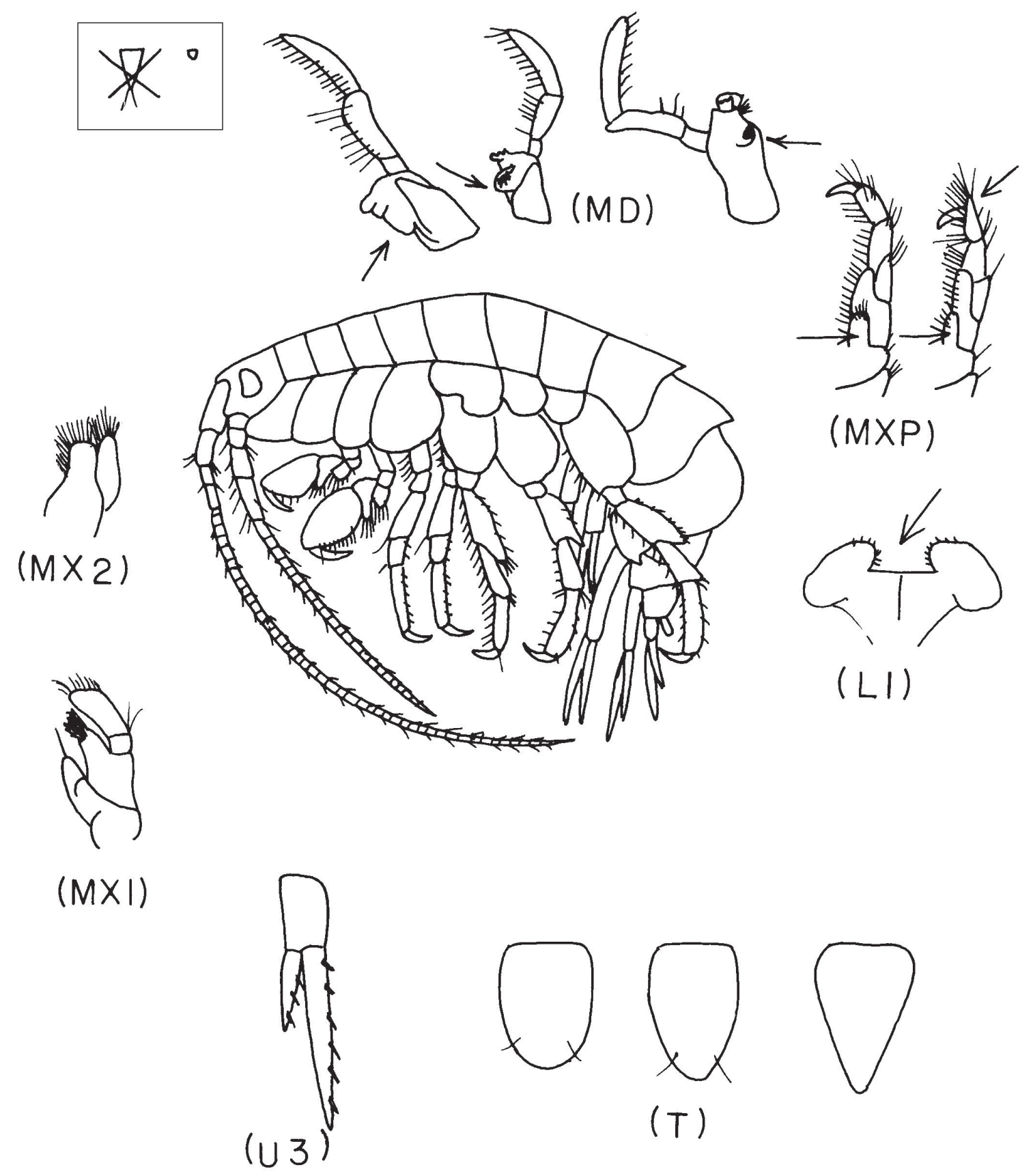

Lámina 63.- Familila PLEUSTIDAE (continúa). 

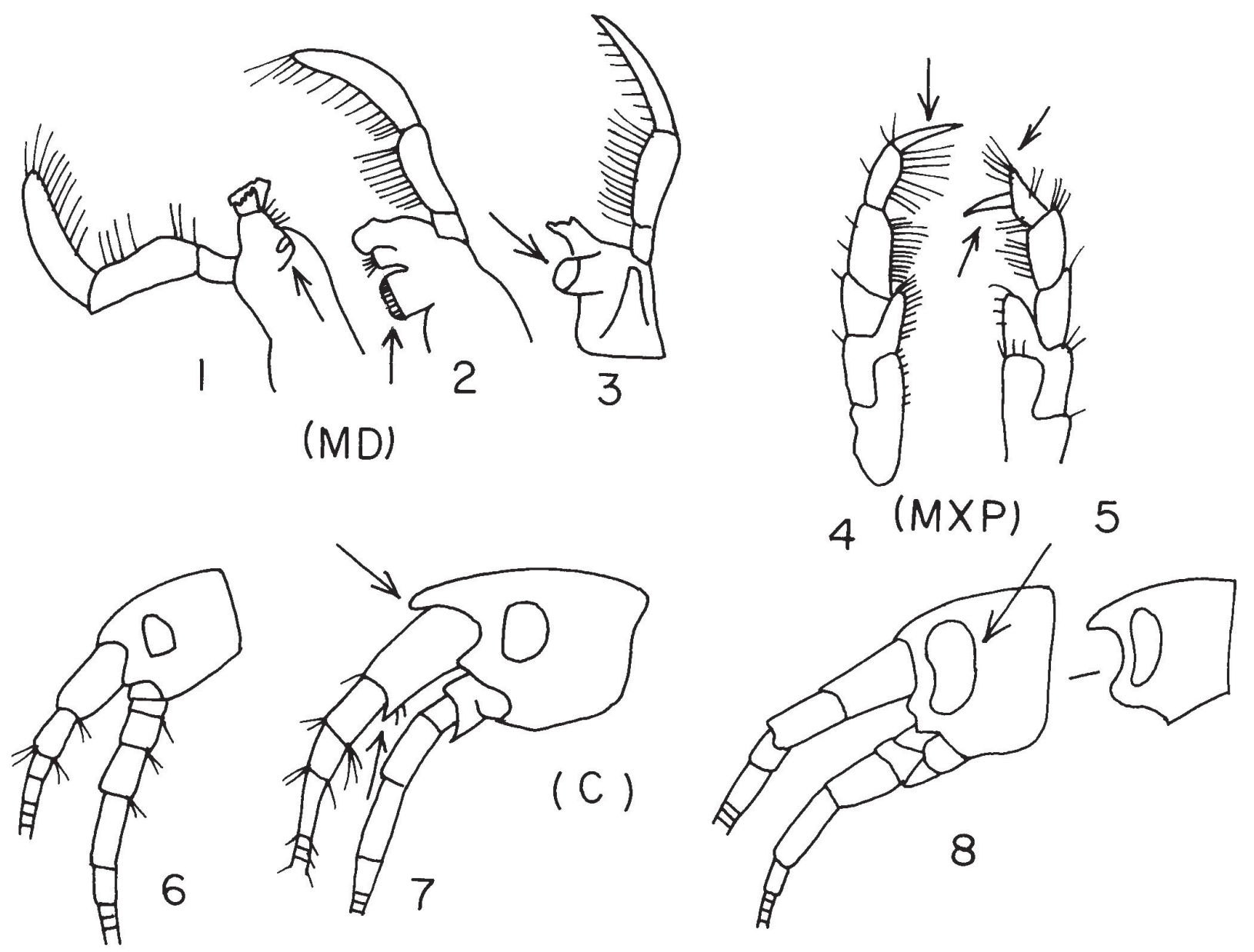

Lámina 64.- Familia PLEUSTIDAE (final).

1, 4, $6 \Rightarrow$ Parapleustes;

3, 4, $7 \Rightarrow$ Pleusymtes;

$2,5,8 \rightarrow$ Stenopleustes. 

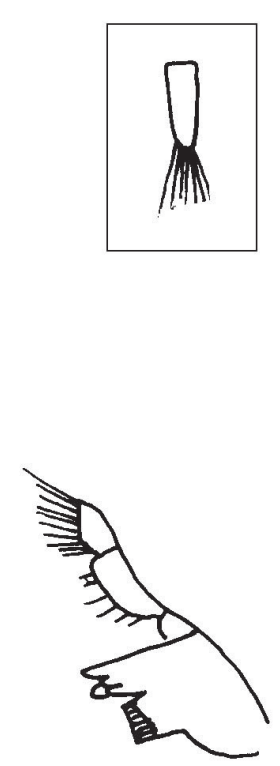

(MD)
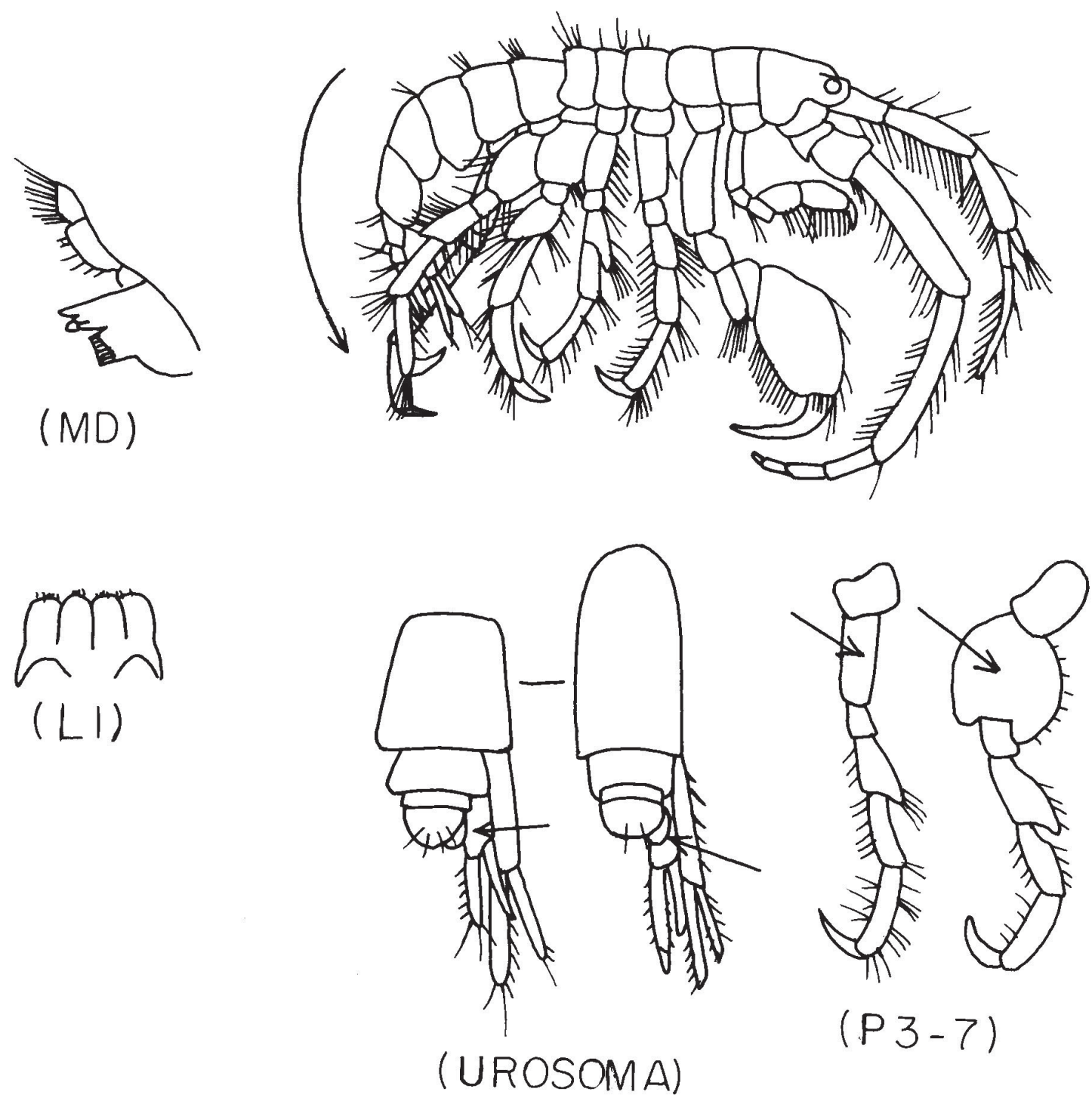

Lámina 65.- Familia PODOCERIDAE (un solo género en el área) Podocerus. 


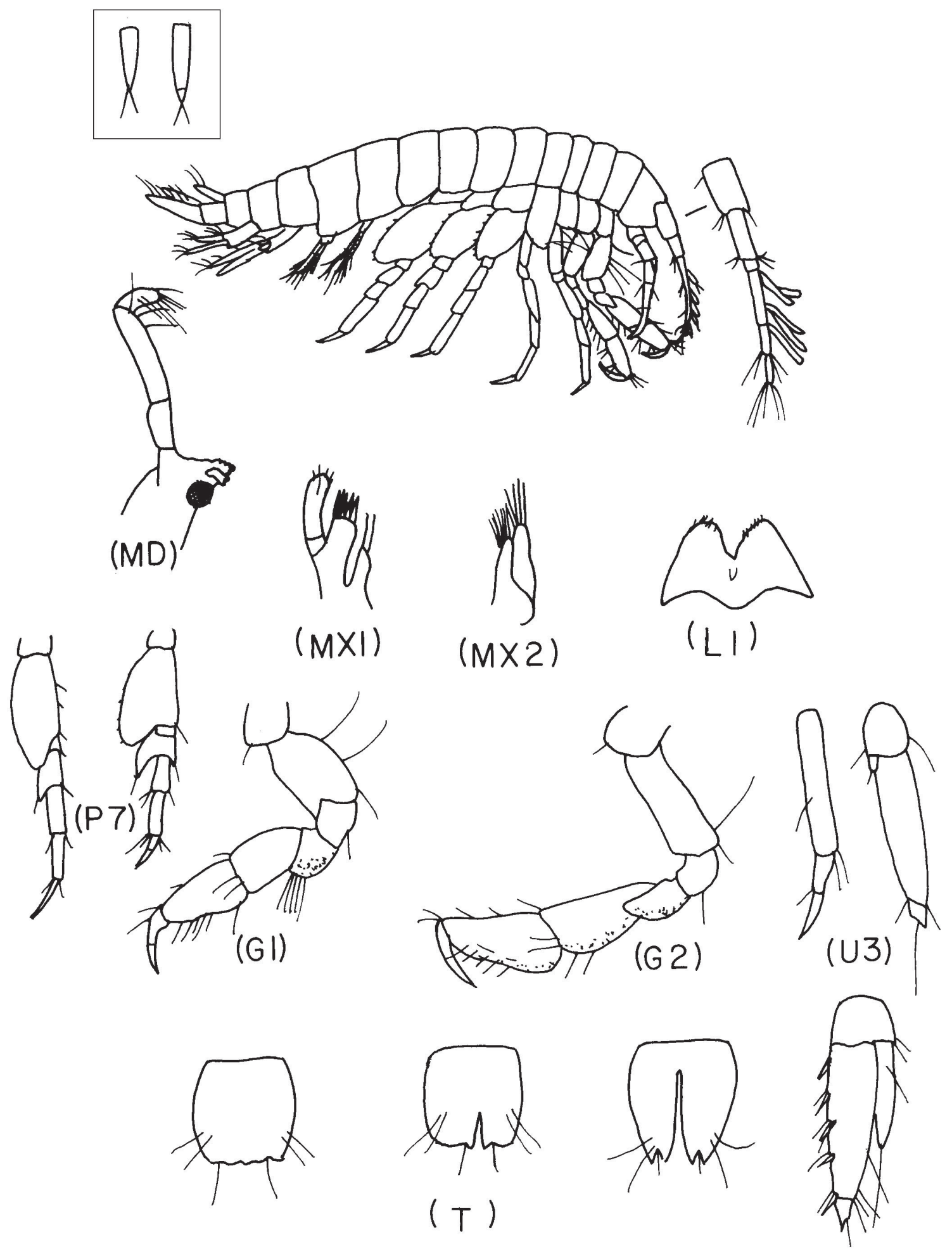

Lámina 66.- Familia SALENTINELLIDAE (un solo género en el área) \$ Salentinella. 

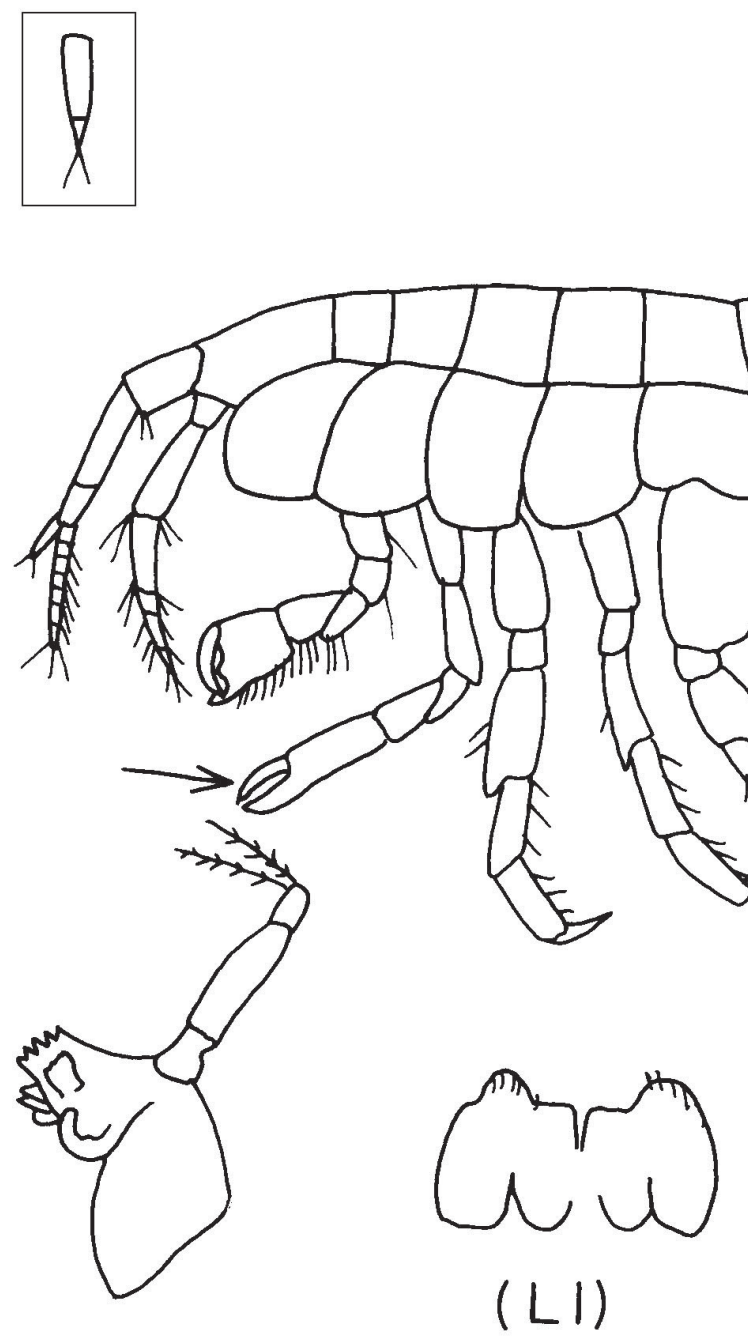

(MD)

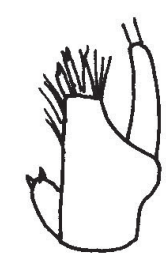

(MXI)
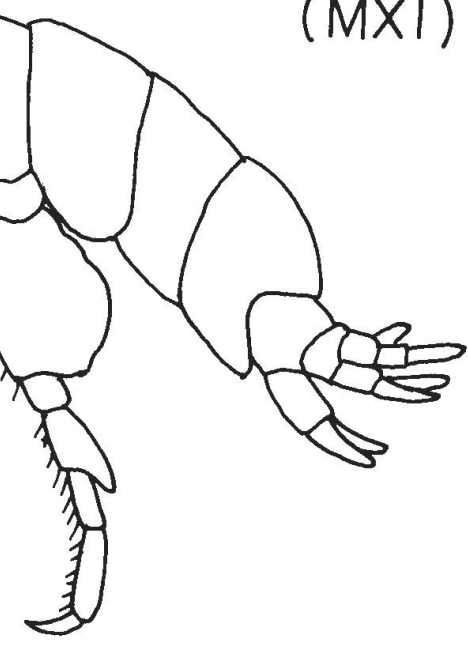

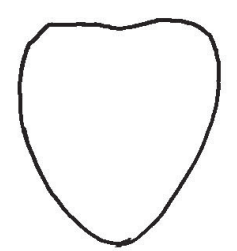

( $\mathrm{T}$ )

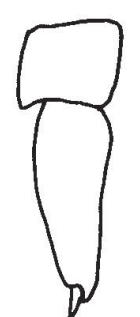

(U3)

Lámina 67.- Familia SEBIDAE (un solo género en el área) $\rightarrow$ Seba. 

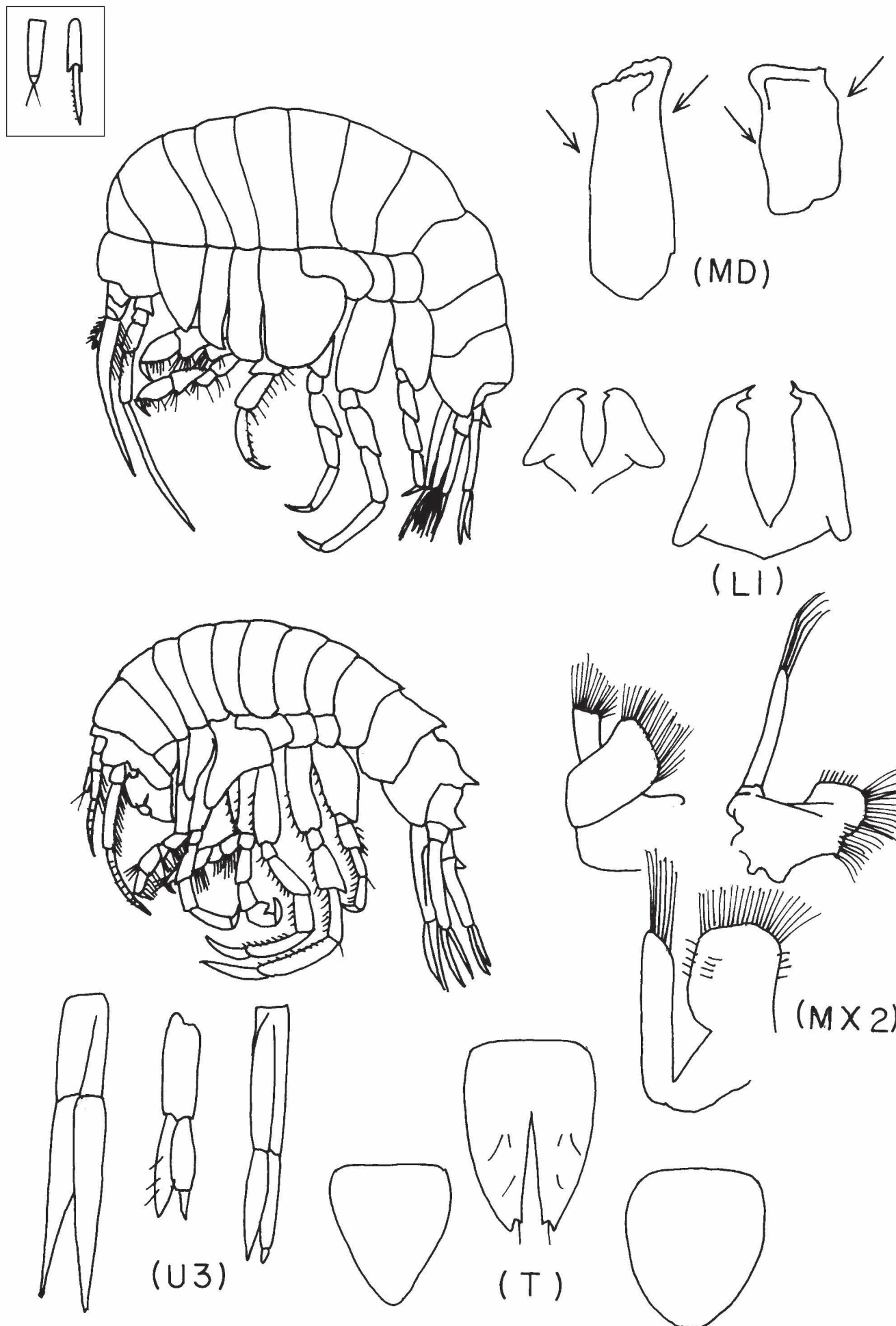

( LI)

Lámina 68. - Familia STEGOCEPHALIDAE (continúa). 


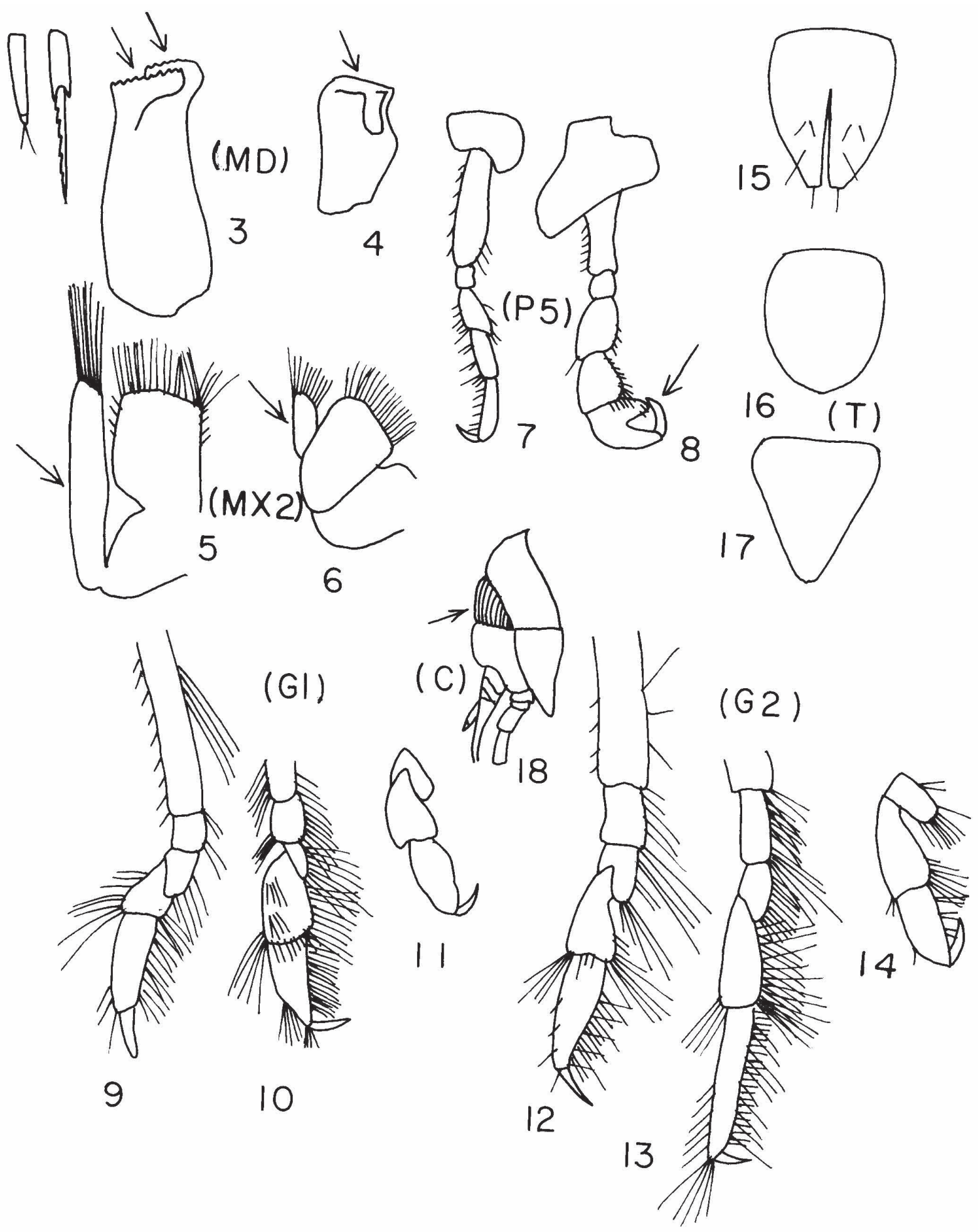

Lámina 69.- Familia STEGOCEPHALIDAE (final).

$1,4,6,7,11,14,16,18 \Rightarrow$ Parandania;

1, 4, 6, 8, 10, 13, $17 \rightarrow$ Parandaniexis;

2, 3, 5, 7, 9, 12, $15 \div$ Stegocephaloides. 

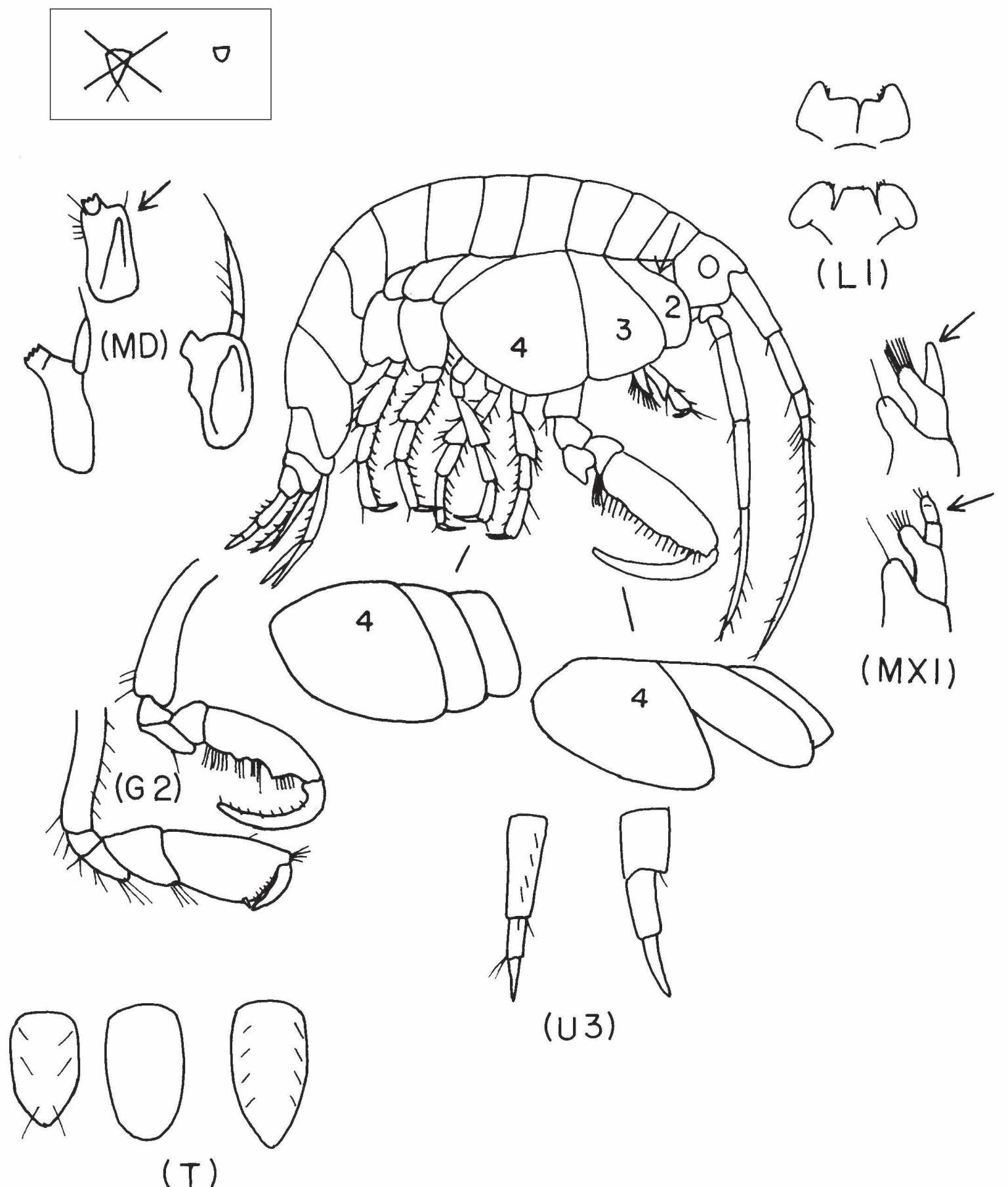

(U3)

Lámina 70.—Familia STENOTHOIDAE (continúa). 


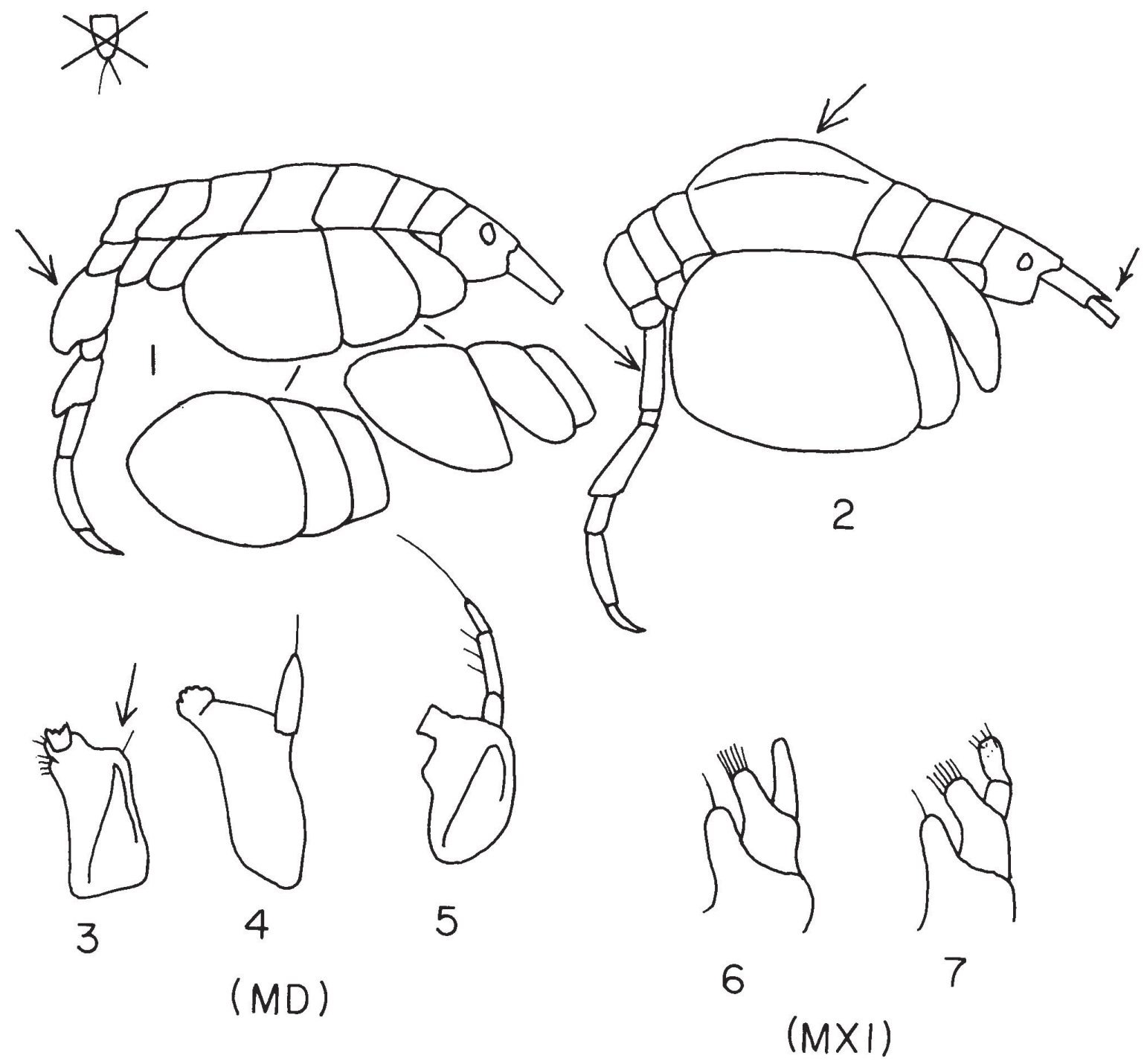

Lámina 71. - Familia STENOTHOIDAE (final).

2, $6 \Rightarrow$ Hardametopa;

1, 3, $6 \Rightarrow$ Parametopa;

1, 5, $7 \rightarrow$ Proboloides;

1, 3, 7 Stenothoe. 


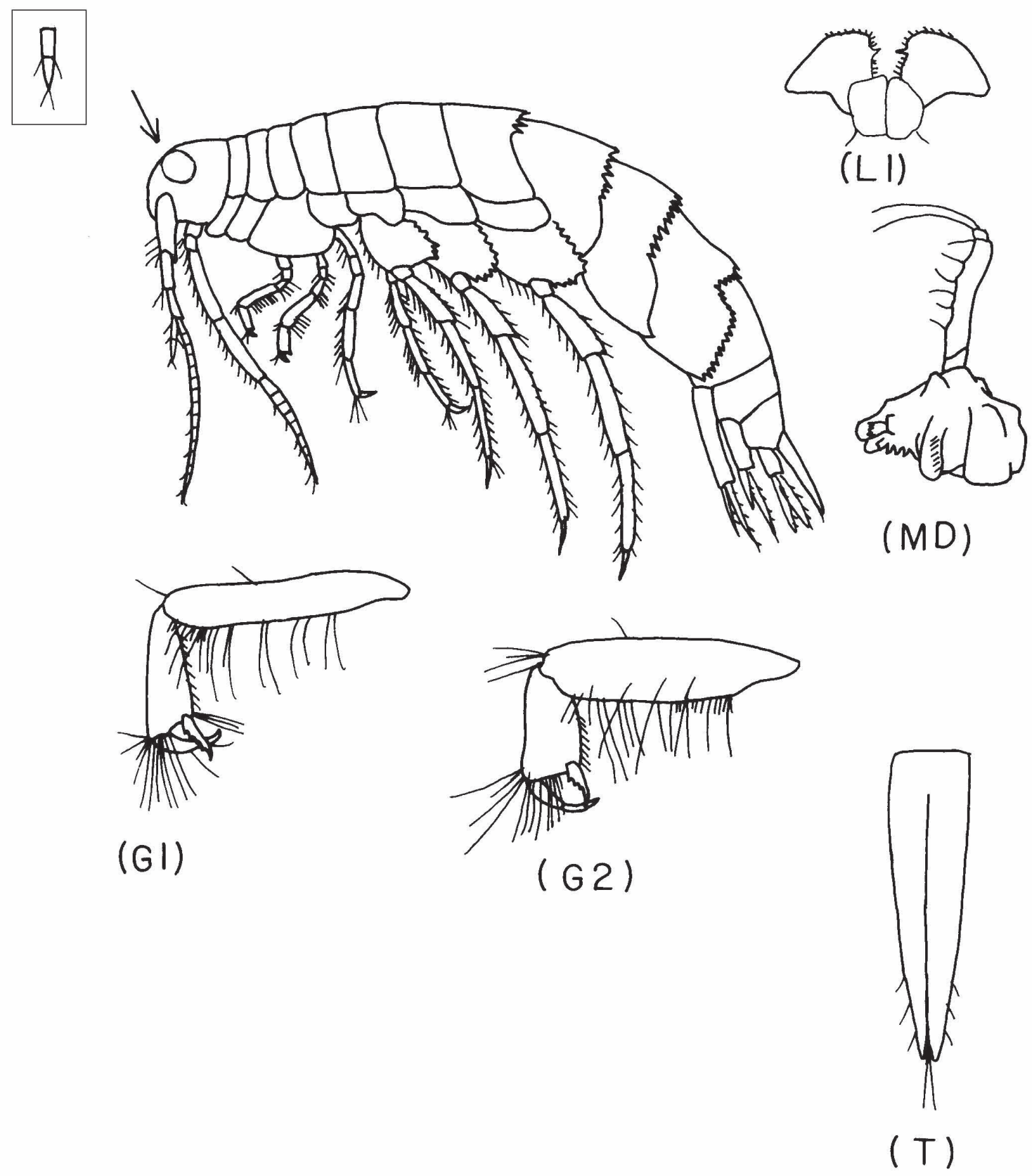

Lámina 72.—Familia SYNOPIIDAE (un solo género en el área) $\rightarrow$ Syrrhoe. 

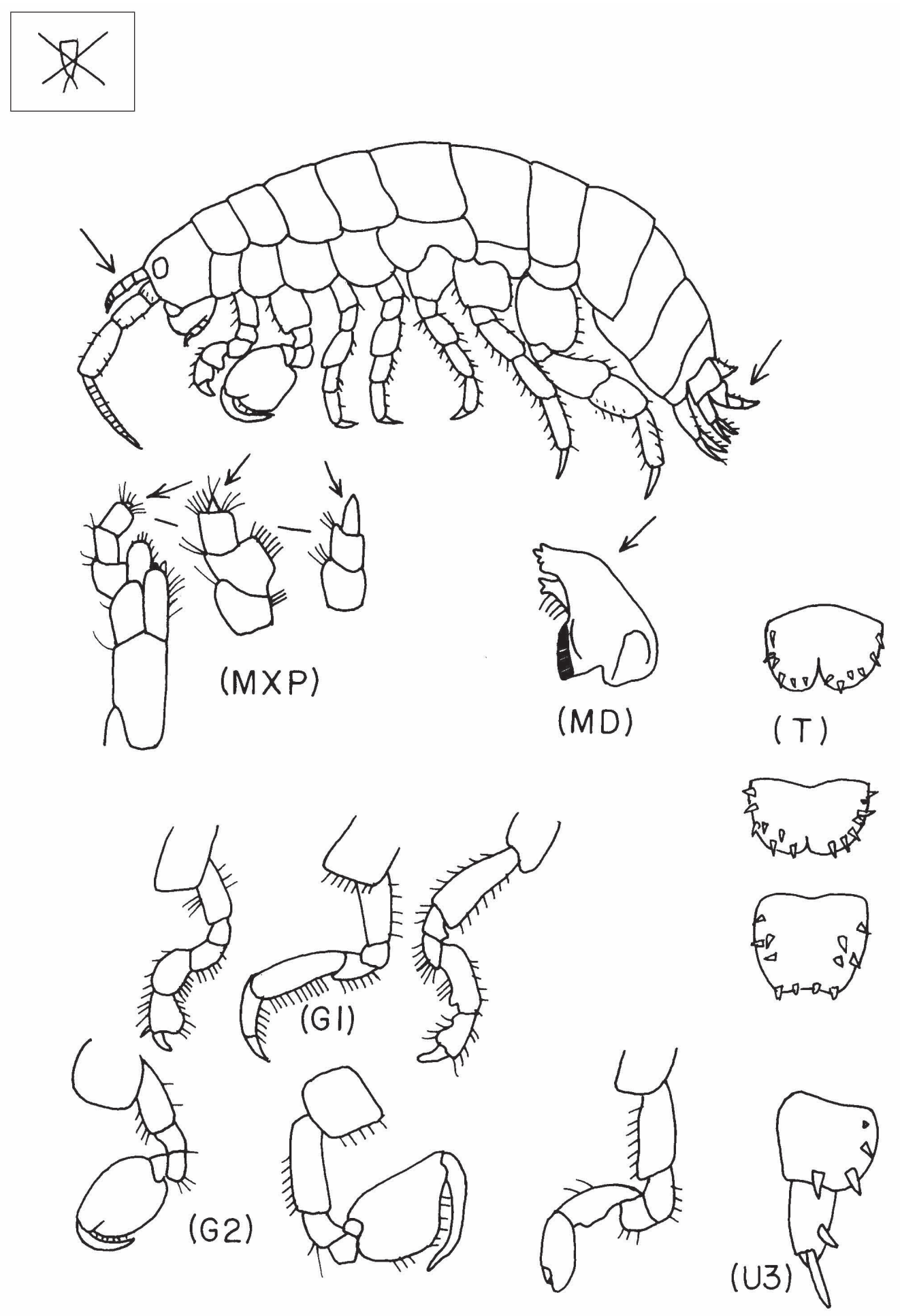

Lámina 73.— Familia TALITRIDAE (continúa). 


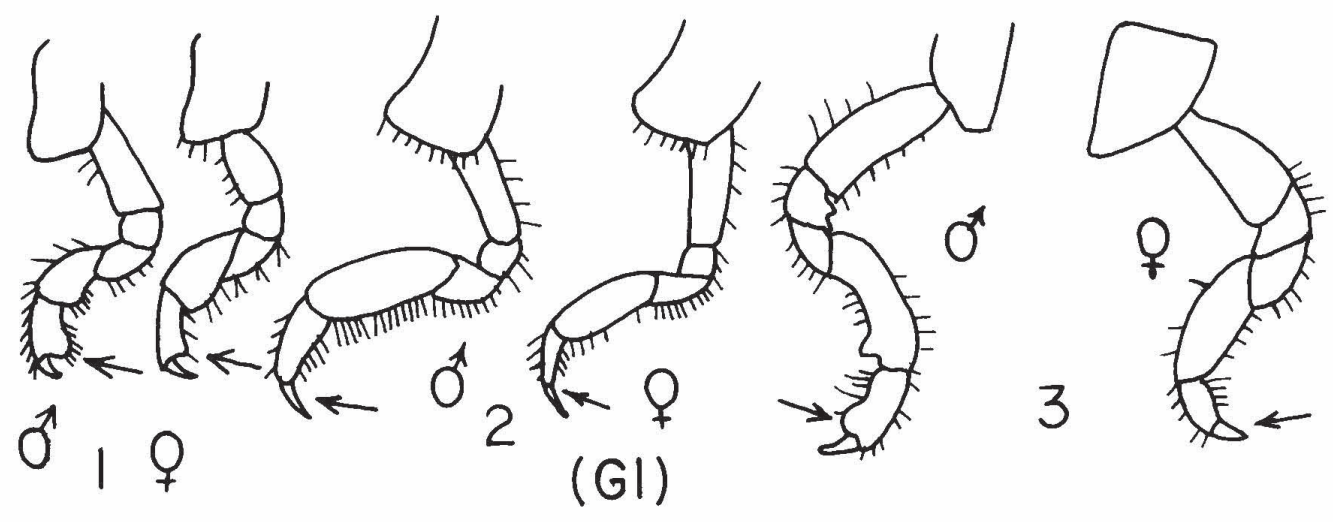

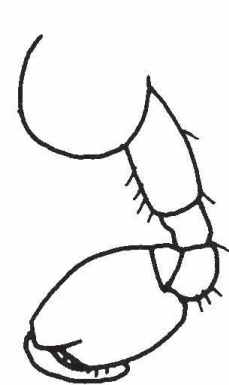

o 4 \%

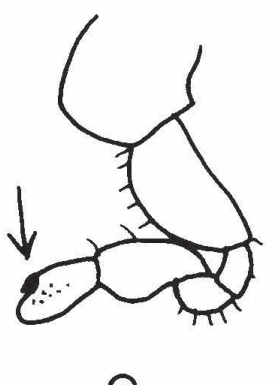

(G2)
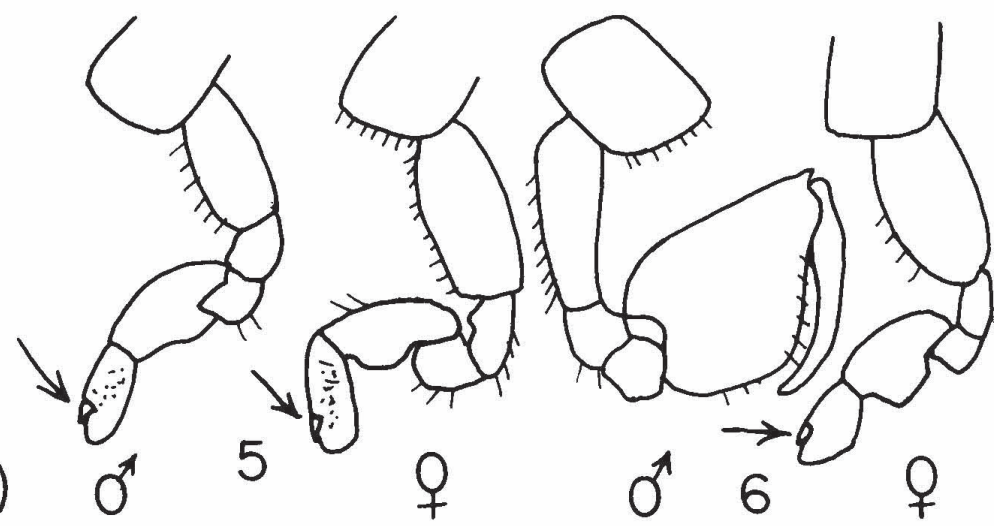

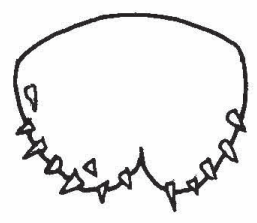

7

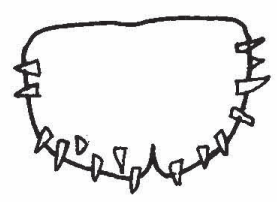

8

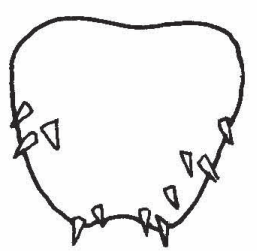

9

Lámina 74.- Familia TALITRIDAE (final).

1, 4, $7 \Rightarrow$ Orchestia:

2, 5, $8 \Rightarrow$ Talitrus;

3, 6,9 $\Rightarrow$ Talorchestia. 


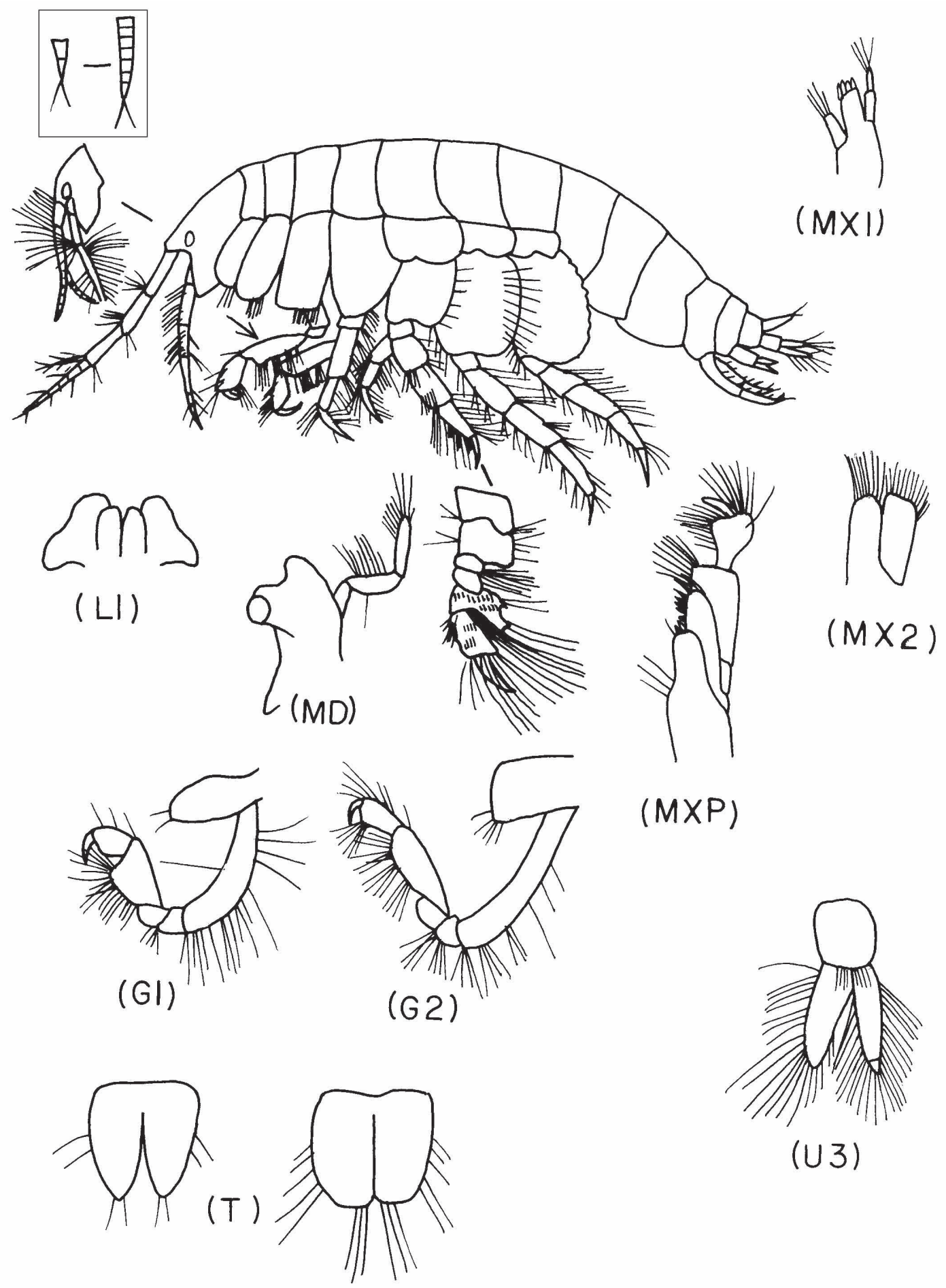

Lámina 75.- Familia UROTHOIDAE (un solo género en el área) $\Rightarrow$ Urothoe (ver HAUSTORIDAE). 


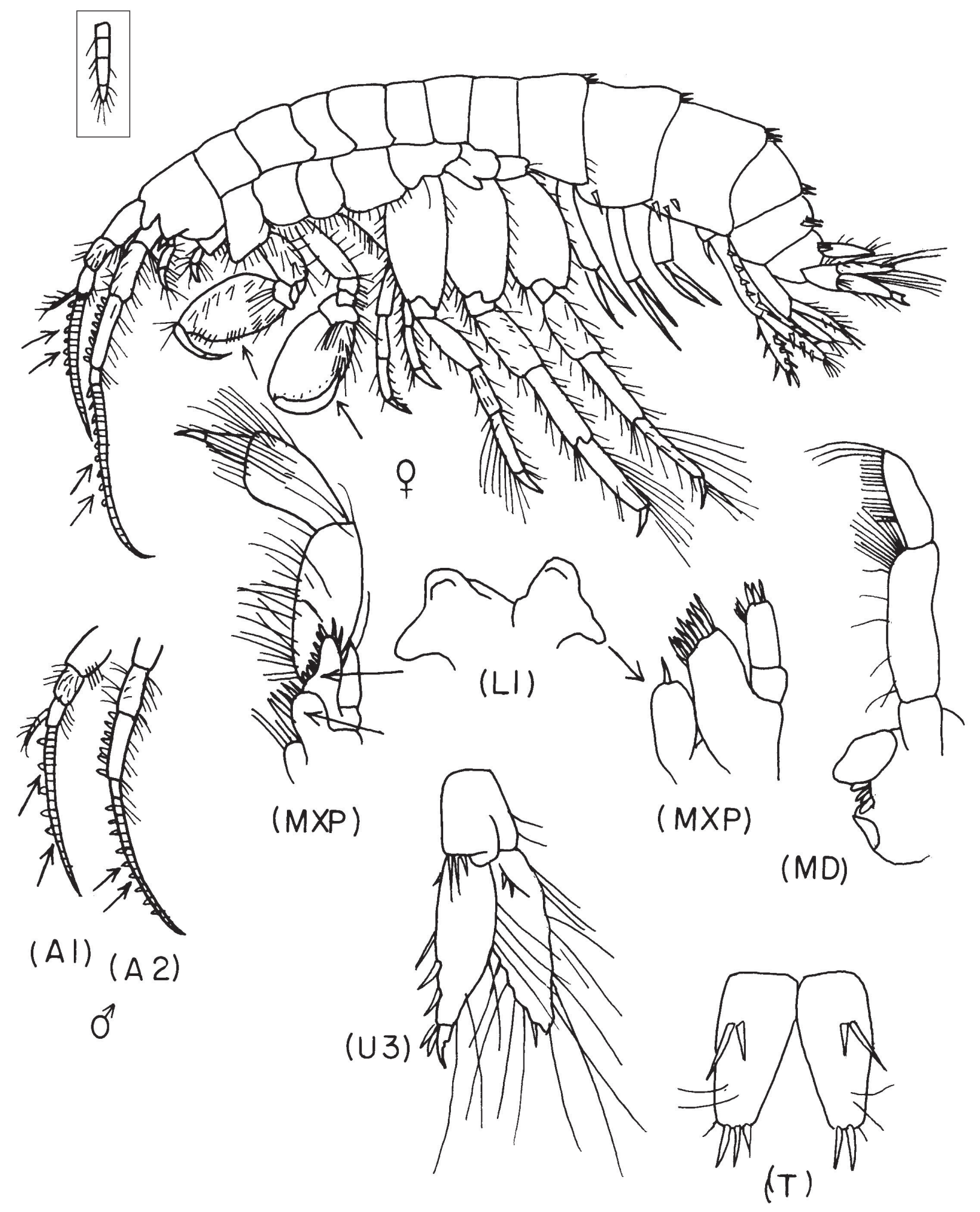

